\title{
Rh-catalyzed asymmetric hydroaminomethylation of $\alpha$ - substituted acrylamides: application in the synthesis of RWAY.
}

Roger Miró, ${ }^{a}$ Anton Cunillera, ${ }^{a}$ Jèssica Margalef, ${ }^{a}$ Domke Lutz, ${ }^{b}$ Armin Börner*,${ }^{b}$ Oscar Pamiès, ${ }^{a}$ Montserrat Diéguez, ${ }^{a}$ Cyril Godard*

${ }^{a}$ Departament de Química Física i Inorgànica, Universitat Rovira I Virgili, C/ Marcel·lí Domingo 1, 43007, Tarragona, Spain.

${ }^{b}$ Leibniz-Institut für Katalyse e.V.Universität Rostock. Albert-Einstein-Strasse 29a, 18059 Rostock, Germany. 


\section{Table of contents}

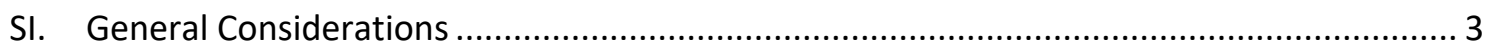

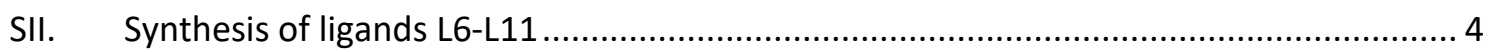

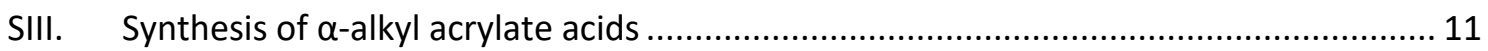

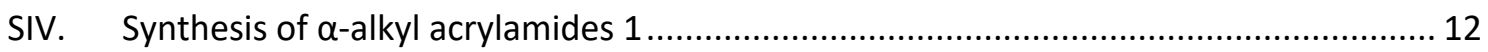

SV. General procedure for Rh-catalyzed Asymmetric HF of $\alpha$-alkyl acrylamides ................. 15

SVI. Optimization of the reaction conditions in the Rh-catalyzed AHF.............................. 16

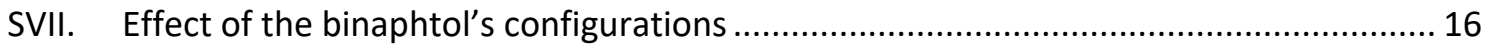

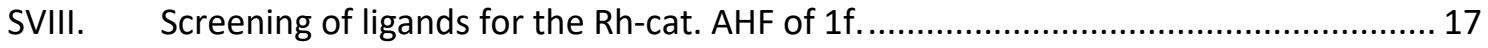

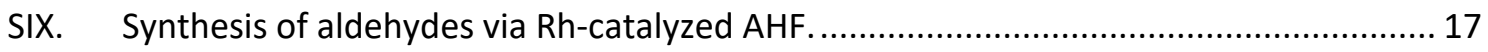

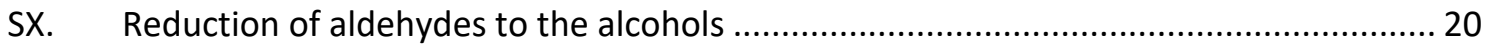

SXI. General procedure for Rh-catalyzed asymmetric hydroaminomethylation of $\alpha$-alkyl

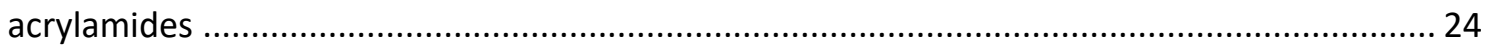

SXII. Optimization of the reaction conditions in the Rh-catalyzed HAM ................................ 25

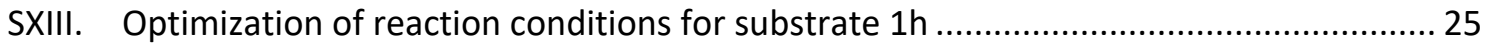

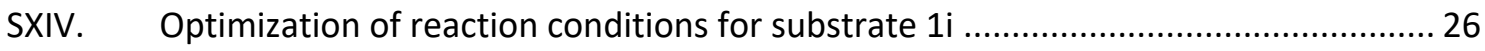

SXV. Optimization of the reaction conditions for the synthesis of RWAY (4I) ........................ 26

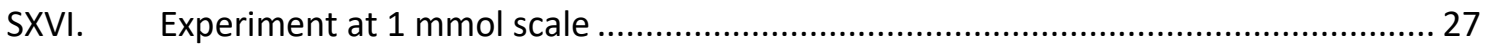

SXVII. Synthesis of $\alpha$-alkyl- $\gamma$-aminobutyric amides via Rh-catalyzed asymmetric HAM....... 27

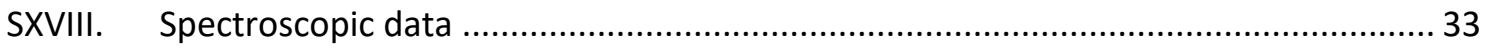

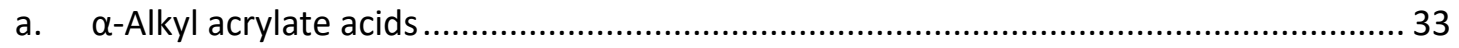

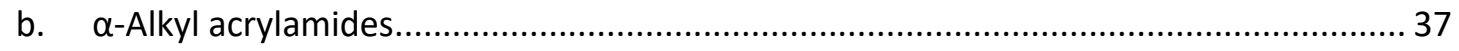

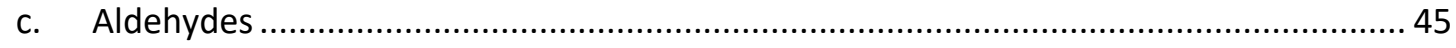

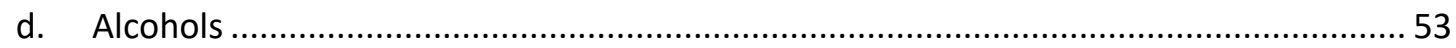

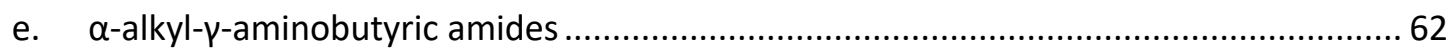

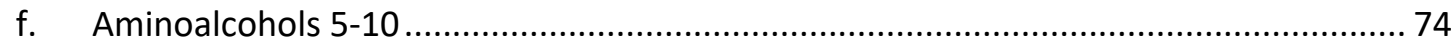

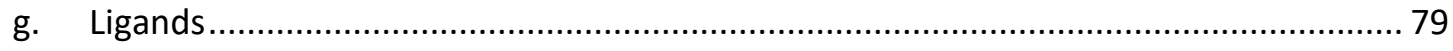

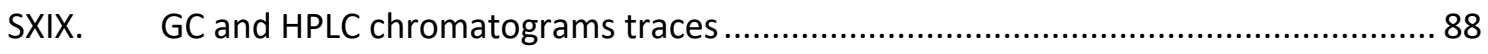

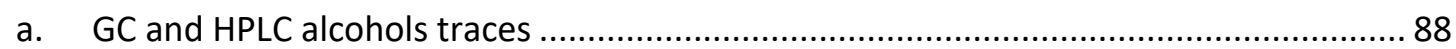

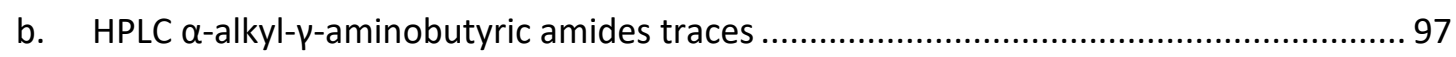

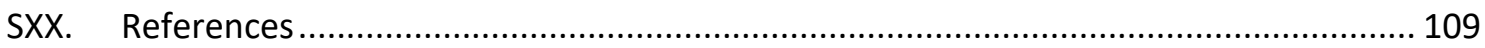




\section{SI. General Considerations}

General: All the reactions were carried out using Schlenk-line inert atmosphere techniques or glovebox techniques. Anhydrous solvents were collected from the system Braun MB SPS-800 except from 1,2-dichloroethane, which was dried over $\mathrm{CaH}_{2}$, and stored under inert atmosphere. 1. For reactions that required heating, the heat source used was an oil bath.

Reagents: Commercially available reagents and solvents were purchased at the highest commercial quality from Sigma-Aldrich and were used as received.

The ligands L1, L2, L3 and L4 were purchased from Strem Chemicals and the L5 was synthetized as previously reported by our group ${ }^{1}$.

Analytical methods: ${ }^{1} \mathrm{H},{ }^{31} \mathrm{P}\left\{{ }^{1} \mathrm{H}\right\}$ and ${ }^{13} \mathrm{C}\left\{{ }^{1} \mathrm{H}\right\}$ NMR spectra were recorded using a Varian Mercury VX 400 (400 and 100.6 MHz respectively). Chemical shift values $(\delta)$ are reported in ppm relative to TMS $\left({ }^{1} \mathrm{H}\right.$ and $\left.{ }^{13} \mathrm{C}\left\{{ }^{1} \mathrm{H}\right\}\right)$ and coupling constants are reported in Hertz. The following abbreviations are used to indicate the multiplicity: s, singlet; d, doublet; t, triplet; q, quartet; quint, quintuplet; sext, sextuplet; sept, septet; oct, octet; m, multiplet; bs, broad signal. High-resolution mass spectra (HRMS) were recorded on an Agilent Time-of-Flight 6210 using ESI-TOF (electrospray ionization-time of flight). Samples were introduced to the mass spectrometer ion source by direct injection using a syringe pump and were externally calibrated using sodium formate. The instrument was operating in the positive ion mode. Reactions were monitored by TLC carried out on $0.25 \mathrm{~mm}$ E. Merck silica gel $60 \mathrm{~F}_{254}$ glass or aluminum plates. Developed TLC plates were visualized under a short-wave UV lamp $(254 \mathrm{~nm})$ and by heating plates that were dipped in potassium permanganate. Flash column chromatography was carried out using forced flow of the indicated solvent on Merck silica gel 60 (230-400 mesh), neutral aluminium oxide $(50-200 \mathrm{~nm}, 60 \mathrm{~A})$ or florisil ${ }^{\circledR}(60-100$ mesh).

The enantiomeric excess of the linear alcohols $\mathbf{5}$ (after reduction of the corresponding aldehydes 2) and the $\mathrm{Y}$-aminobutyric amides 3 were determined by HPLC analysis employing Daicel Chiralpak IA, ID, IF, IC or OD-H chiral columns, or GC analysis employing CP-ChiraSil -DEX CB $25 \times 0.25$ column. The exact conditions for the analyses are specified within the supporting information section. HPLC and GC traces were compared to racemic samples prepared performing the reactions in the presence of ditert-butylphenylphosphine or 1,3,5,7-tetramethyl-8-phenyl-2,4,6-trioxa-8phosphaadamantane. Optical rotations $[\alpha]_{D}$ were measured on a PERKIN ELMER 
polarimeter 241 instrument. Melting points were measured on a Stuart Scientific SMP3 melting point apparatus.

Catalysis: The Rh-catalyzed HF and HAM reaction was set up in a CAT24 autoclave from HEL Inc. and was stirred with a teflon-coated magnetic stir bar.

\section{SII. Synthesis of ligands L6-L11}

The ligands have been prepared from D-xylose. ${ }^{4}$ The synthetic reactions involved for the preparation of key amino-alcohol intermediates 5-10 (see Supporting Information) is classical well-established old sugar synthetic chemistry, which do not imply any modification in the configuration of the chiral center of D-xylose.
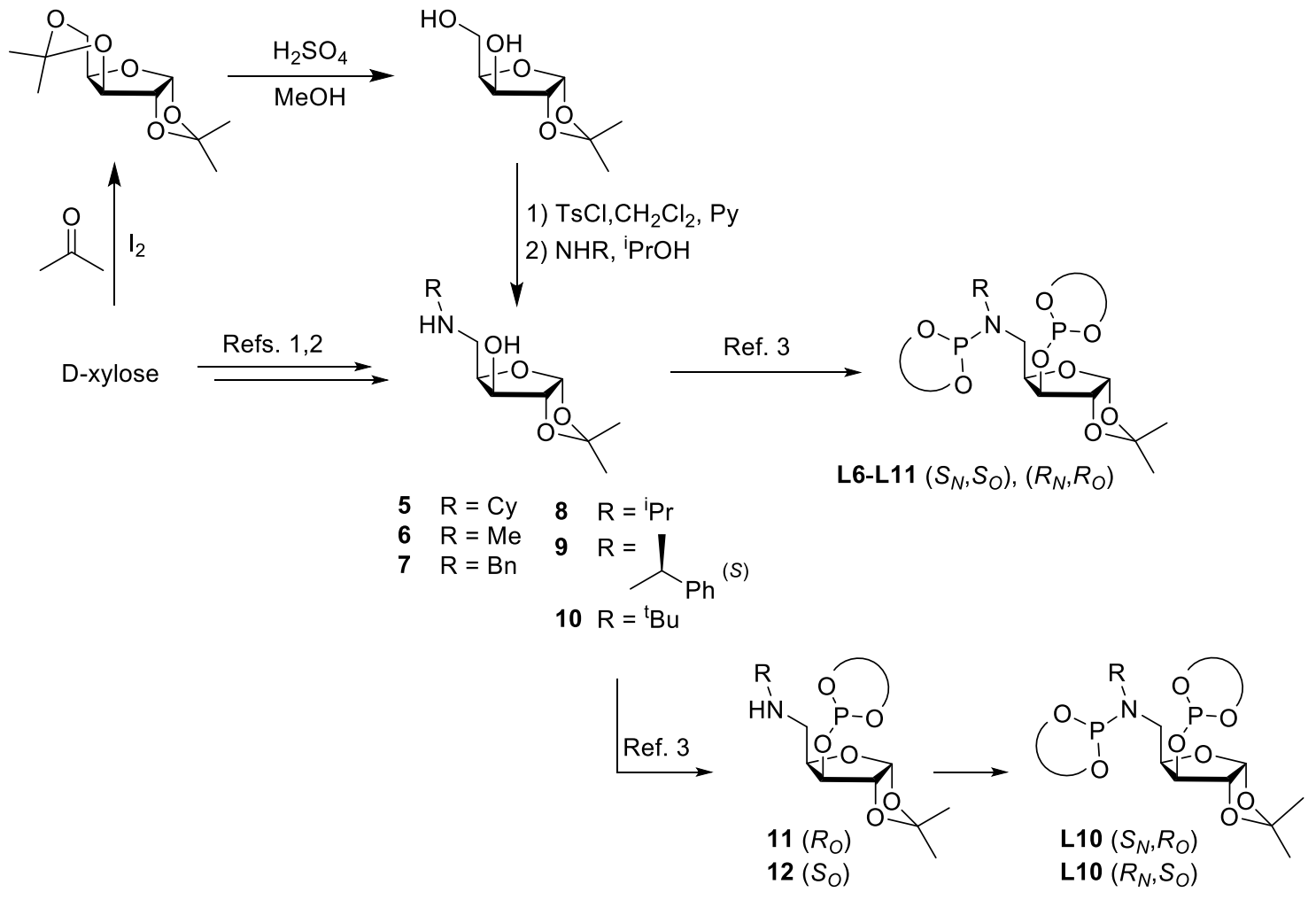

General procedure for the preparation of aminoalcohols 5, 7 and 9.

1,2-O-isopropylidene-5-O-tosyl- $\alpha$-D-xylofuranose ${ }^{2}(1.0 \mathrm{mmol})$ and the corresponding amine $(4.0 \mathrm{mmol})$ were dissolved in isopropanol $(2 \mathrm{~mL})$. The mixture was heated in an oil bath to reflux and stirred for $24 \mathrm{~h}$. After cooling to room temperature, it was concentratetd in vacuo. The residue was treated with saturated $\mathrm{NaHCO}_{3}$-solution and extracted with diethyl ether (three times). The combined organic phases were dried with $\mathrm{Na}_{2} \mathrm{SO}_{4}$, concentrated in vacuo and purified through $\mathrm{SiO}_{2}$-column chromatography $\left(\mathrm{EtOAc} / \mathrm{Et}_{3} \mathrm{~N}=9: 1\right)$ to give aminoalcohols $5,{ }^{3} \mathbf{7}^{3}$ and $\mathbf{9}$.

5-Cyclohexylamino-5-deoxy-1,2-O-isopropylidene-a-D-xylofuranose (5) ${ }^{3}$ 
Starting from cyclohexylamine $(4.00 \mathrm{~g}, 40.0 \mathrm{mmol})$ and 1,2-O-isopropylidene-5-O-tosyla-D-xylofuranose $(3.45 \mathrm{~g}, 10.0 \mathrm{mmol})$ in isopropanol $(20 \mathrm{~mL})$, product 5 was isolated through $\mathrm{SiO}_{2}$-column chromatography $\left(\mathrm{EtOAc} / \mathrm{Et}_{3} \mathrm{~N}=9: 1\right)$ as an off-white solid $(1.70 \mathrm{~g}$, $63 \%) .{ }^{1} \mathrm{H} \mathrm{NMR}\left(\mathrm{CDCl}_{3}, 250 \mathrm{MHz}\right): \delta=5.95(\mathrm{~d}, 1 \mathrm{H}, J=3.7 \mathrm{~Hz}), 4.48(\mathrm{~d}, 1 \mathrm{H} \mathrm{J}=3.7 \mathrm{~Hz})$, $4.27(\mathrm{~d}, 1 \mathrm{H}, J=2.9 \mathrm{~Hz}), 4.22-4.19(\mathrm{~m}, 1 \mathrm{H}), 3.46(\mathrm{dd}, 1 \mathrm{H}, J=12.9 \mathrm{~Hz}, J=3.5 \mathrm{~Hz}), 3.00$ (dd, $1 \mathrm{H}, J=12.9 \mathrm{~Hz}, J=1.3 \mathrm{~Hz}), 2.46-2.35(\mathrm{~m}, 1 \mathrm{H}), 1.92-1.82(\mathrm{~m}, 2 \mathrm{H}), 1.74-1.68(\mathrm{~m}$, $2 \mathrm{H}), 1.61-1.55(\mathrm{~m}, 1 \mathrm{H}), 1.47(\mathrm{~s}, 3 \mathrm{H}), 1.31(\mathrm{~s}, 3 \mathrm{H}), 1.29-0.98(\mathrm{~m}, 5 \mathrm{H}) .{ }^{13} \mathrm{C}\left\{{ }^{1} \mathrm{H}\right\} \quad \mathrm{NMR}$ $\left(\mathrm{CDCl}_{3}, 63 \mathrm{MHz}\right): \delta=111.4,105.1,86.1,78.3,77.0,56.3,45.5,33.1,32.8,26.8,26.2$, 25.9, 24.7, 24.7.

\section{5-Benzylamino-5-deoxy-1,2-0-isopropylidene- $\alpha-D-x y l o f u r a n o s e ~(7)^{3}$}

Starting from benzylamine $(4.29 \mathrm{~g}, 40.0 \mathrm{mmol})$ and 1,2-O-isopropylidene-5-O-tosyl- $\alpha-\mathrm{D}$ xylofuranose $(3.45 \mathrm{~g}, 10.0 \mathrm{mmol})$ in isopropanol $(20 \mathrm{~mL})$, product 7 was isolated through $\mathrm{SiO}_{2}$-column chromatography (EtOAc/Et $\left.3 \mathrm{~N}=9: 1\right)$ as an off-white solid $(1.70 \mathrm{~g}, 61 \%) .{ }^{1} \mathrm{H}$ $\operatorname{NMR}\left(\mathrm{CDCl}_{3}, 400 \mathrm{MHz}\right): \delta=7.26-7.18(\mathrm{~m}, 5 \mathrm{H}), 5.86(\mathrm{~d}, 1 \mathrm{H}, J=4.0 \mathrm{~Hz}), 4.42(\mathrm{~d}, 1 \mathrm{H}, J$ $=3.2 \mathrm{~Hz}), 4.19(\mathrm{~d}, 1 \mathrm{H}, J=2.4 \mathrm{~Hz}), 4.13(\mathrm{~b}, 1 \mathrm{H}), 3.71(\mathrm{~d}, 1 \mathrm{H}, J=13.2 \mathrm{~Hz}), 3.65(\mathrm{~d}, 1 \mathrm{H}, J$ $=13.2 \mathrm{~Hz}), 3.27(\mathrm{dd}, 1 \mathrm{H}, J=12.8 \mathrm{~Hz}, J=3.2 \mathrm{~Hz}), 2.89(\mathrm{dd}, 1 \mathrm{H}, J=12.8 \mathrm{~Hz}, J=1.2 \mathrm{~Hz})$, 1.40 (s, 3H), 1.24 (s, 3H). ${ }^{13} \mathrm{C}\left\{{ }^{1} \mathrm{H}\right\}$ NMR $\left(\mathrm{CDCl}_{3}, 75 \mathrm{MHz}\right): \delta=139.0,129.1,128.7,128.0$, $111.9,105.5,86.3,78.5,77.3,54.0,48.1,27.0 .26 .3$.

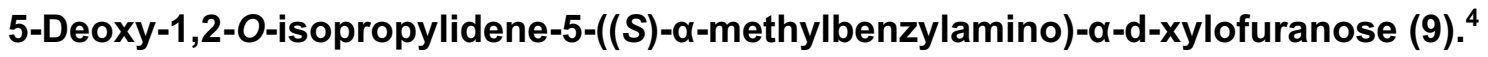

Starting from (S)-a-methylbenzylamine (4.85 g, $40.0 \mathrm{mmol})$ and 1,2-O-isopropylidene-5O-tosyl-a-D-xylofuranose $(3.45 \mathrm{~g}, 10.0 \mathrm{mmol})$ in isopropanol $(20 \mathrm{~mL})$, product 9 was isolated through $\mathrm{SiO}_{2}$-column chromatography $\left(\mathrm{EtOAc} \mathrm{Et} \mathrm{t}_{3} \mathrm{~N}=9: 1\right)$ as a yellowish viscous oil $(2.90 \mathrm{~g}, 99 \%)$. ${ }^{1} \mathrm{H}$ NMR $\left(\mathrm{CDCl}_{3}, 250 \mathrm{MHz}\right): \delta=7.38-7.20(\mathrm{~m}, 29 \mathrm{H}), 5.95(\mathrm{~d}, 1 \mathrm{H}, J=$ $3.7 \mathrm{~Hz}), 4.51(\mathrm{~d}, 1 \mathrm{H}, J=3.7 \mathrm{~Hz}), 4.30(\mathrm{~d}, 1 \mathrm{H}, J=2.9 \mathrm{~Hz}), 4.17-4.20(\mathrm{~m}, 1 \mathrm{H}), 3.72(\mathrm{q}, 1 \mathrm{H}$, $J=6.7 \mathrm{~Hz}), 3.18(\mathrm{dd}, 1 \mathrm{H}, J=12.9 \mathrm{~Hz}, J=3.8 \mathrm{~Hz}), 2.90(\mathrm{dd}, 1 \mathrm{H}, J=12.9 \mathrm{~Hz}, J=1.4$ $\mathrm{Hz}), 1.46$ (s, 3H), 1.39 (dd, 3H, J = 6.7 Hz), $1.32(\mathrm{~s}, 3 \mathrm{H}) \cdot{ }^{13} \mathrm{C}\left\{{ }^{1} \mathrm{H}\right\} \mathrm{NMR}\left(\mathrm{CDCl}_{3}, 63 \mathrm{MHz}\right)$ : $\delta=143.7,128.7,127.4,126.1,111.4,105.0,85.9,77.2,76.9,58.1,46.3,26.8,26.1$, 23.2. Anal. calcd for $\mathrm{C} 16 \mathrm{H} 23 \mathrm{NO}$ : $\mathrm{C}, 65.51 ; \mathrm{H}, 7.90 ; \mathrm{N}, 4.77$. Found: $\mathrm{C}, 65.20 ; \mathrm{H}, 8.01$; $\mathrm{N}, 4.95 \%$. HRMS (ESI) calculated for C16H24NO4 294.16998, found 294.16984. HRMS (ESI) calculated for $\mathrm{C} 16 \mathrm{H} 23 \mathrm{NO} 4 \mathrm{Na} 316.15193$, found 316.15178.

\section{General procedure for the preparation of aminoalcohols 6, 8 and 10.}

1,2-O-isopropylidene-5-O-tosyl- $\alpha$-D-xylofuranose $(1.0 \mathrm{mmol})$ and the corresponding alkyl amine $(2 \mathrm{~mL})$ were stirred in a pressure tube at $60^{\circ} \mathrm{C}$ (heated in an oil bath) for 24 $\mathrm{h}$. The reaction mixture was cooled to room temperature and concentrated in vacuo. The 
residue was taken up in dichloromethane $(50 \mathrm{~mL})$, washed with saturated $\mathrm{NaHCO}_{3}$ solution, water and finally with brine (each $20 \mathrm{~mL}$ ). The organic phase was dried over $\mathrm{Na}_{2} \mathrm{SO}_{4}$, concentrated in vacuo and purify through $\mathrm{SiO}_{2}$-column chromatography $\left(\mathrm{EtOAc} / \mathrm{Et}_{3} \mathrm{~N}=97: 3\right)$ to give aminoalcohols 6,8 and $10 .^{3}$

\section{5-Deoxy-5-methylamino-1,2-0-isopropylidene- $\alpha-D-x y l o f u r a n o s e ~(6)^{3}$.}

Starting from methylamine $(40 \% \mathrm{w} / \mathrm{w}$ aqueous solution) $(10.0 \mathrm{ml})$ and $1,2-0-$ isopropylidene-5-O-tosyl- $\alpha-D-x y l o f u r a n o s e ~(1.75 \mathrm{~g}, 5 \mathrm{mmol})$, product 8 was isolated through $\mathrm{SiO}_{2}$-column chromatography (EtOAc/Et ${ }_{3} \mathrm{~N}=97: 3$ )as an off-white solid (492.3 mg, 48 \%). ${ }^{1} \mathrm{H}$ NMR $\left(\mathrm{CDCl}_{3}, 400 \mathrm{MHz}\right): \delta=5.91(\mathrm{~d}, 1 \mathrm{H}, J=4.0 \mathrm{~Hz}), 4.46(\mathrm{~d}, 1 \mathrm{H}, J=3.6$ $\mathrm{Hz}$ ), $4.25(\mathrm{~d}, 1 \mathrm{H}, J=3.2 \mathrm{~Hz}), 4.18-4.16(\mathrm{~m}, 1 \mathrm{H}), 3.33(\mathrm{dd}, 1 \mathrm{H}, J=13.2 \mathrm{~Hz}, J=3.6 \mathrm{~Hz})$, 2.89 (dd, $1 \mathrm{H}, J=12.8 \mathrm{~Hz}, J=1.2 \mathrm{~Hz}), 2.38(\mathrm{~s}, 3 \mathrm{H}), 1.45(\mathrm{~s}, 3 \mathrm{H}), 1.29(\mathrm{~s}, 3 \mathrm{H})$.

5-Deoxy-5-isopropylamino-1,2-0-isopropylidene- $\alpha-D-x y l o f u r a n o s e ~(8)^{3}$.

Starting from isopropylamine $(20.0 \mathrm{ml})$ and 1,2-O-isopropylidene-5-O-tosyl- $\alpha-D-$ xylofuranose $(3.45 \mathrm{~g}, 10.0 \mathrm{mmol})$, product 8 was isolated through $\mathrm{SiO}_{2}$-column chromatography (EtOAc/Et $3 \mathrm{~N}=97: 3)$ as an off-white solid $(1.70 \mathrm{~g}, 61 \%) .{ }^{1} \mathrm{H}$ NMR $\left(\mathrm{CDCl}_{3}, 250 \mathrm{MHz}\right): \delta=5.95(\mathrm{~d}, 1 \mathrm{H}, J=3.7 \mathrm{~Hz}), 4.48(\mathrm{~d}, 1 \mathrm{H}, J=3.7 \mathrm{~Hz}), 4.28(\mathrm{~d}, 1 \mathrm{H}, J$ $=2.9 \mathrm{~Hz}), 4.23-4.20(\mathrm{~m}, 1 \mathrm{H}), 3.38(\mathrm{dd}, 1 \mathrm{H}, J=12.8 \mathrm{~Hz}, J=3.5 \mathrm{~Hz}), 2.97(\mathrm{dd}, 1 \mathrm{H}, J=$ $12.8 \mathrm{~Hz}, J=1.2 \mathrm{~Hz}), 1.48(\mathrm{~s}, 3 \mathrm{H}), 1.07(\mathrm{~d}, 6 \mathrm{H}, J=6.3 \mathrm{~Hz}), 1.32(\mathrm{~s}, 3 \mathrm{H}), 2.77(\mathrm{~m}, 1 \mathrm{H})$. ${ }^{13} \mathrm{C}\left\{{ }^{1} \mathrm{H}\right\} \mathrm{NMR}\left(\mathrm{CDCl}_{3}, 63 \mathrm{MHz}\right): \delta=111.3,105.1,86.1,78.2,76.9,48.6,46.8,26.8,22.6$, 26.1, 22.3.

\section{5-tert-Butylamino-5-deoxy-1,2-0-isopropylidene- $\alpha$-D-xylofuranose (10) ${ }^{3}$.}

Starting from tert-butylamine $(10 \mathrm{ml})$ and 1,2-O-isopropylidene-5-O-tosyl- $\alpha$-Dxylofuranose $(1.75 \mathrm{~g}, 5.0 \mathrm{mmol})$, product 10 was isolated through $\mathrm{SiO}_{2}$-column chromatography $\left(\mathrm{EtOAc} / \mathrm{Et}_{3} \mathrm{~N}=97: 3\right)$ as an yellowish solid $(1.07 \mathrm{~g}, 87 \%) .{ }^{1} \mathrm{H}$ NMR $\left(\mathrm{CDCl}_{3}, 250 \mathrm{MHz}\right): \delta=5.94(\mathrm{~d}, 1 \mathrm{H}, J=3.7 \mathrm{~Hz}), 4.46(\mathrm{~d}, 1 \mathrm{H}, J=3.7 \mathrm{~Hz}), 4.27(\mathrm{~d}, 1 \mathrm{H}, J=$ $2.9 \mathrm{~Hz}), 4.24-4.21(\mathrm{~m}, 1 \mathrm{H}), 3.35$ (dd, $1 \mathrm{H}, J=12.7 \mathrm{~Hz}, J=3.5 \mathrm{~Hz}), 2.95$ (dd, $1 \mathrm{H}, J=12.7$ $\mathrm{Hz}, J=1.2 \mathrm{~Hz}), 1.31(\mathrm{~s}, 3 \mathrm{H}), 1.47(\mathrm{~s}, 3 \mathrm{H}), 1.10(\mathrm{~s}, 9 \mathrm{H}) .{ }^{13} \mathrm{C}\left\{{ }^{1} \mathrm{H}\right\} \mathrm{NMR}\left(\mathrm{CDCl}_{3}, 63 \mathrm{MHz}\right)$ : $\delta=111.4,105.1,86.1,78.3,76.9,50.2,41.4,28.4,26.9,26.2$.

General procedure for the preparation of phosphite-phosphoramidite ligands L6L11 $\left(S_{N}, S_{o}\right.$ or $\left.R_{o}, R_{N}\right)$. 
2.2 Eq of enantiopure (S)-BINOL were suspended in phosphorus trichloride $(1.5 \mathrm{~mL} / 1.0$ mmol BINOL), 2-3 drops of $\mathrm{N}$-methyl-2-pyrrolidone were added and the solution was heated in an oil bath to $75{ }^{\circ} \mathrm{C}$ for $5 \mathrm{~min}$. The resulting $\mathrm{HCl}$ gas was derived from the reaction vessel by using a bubble counter (slight argon stream!). The now clear solution was cooled to room temperature, concentrated and dried azeotropically with toluene (three times). Thus, the in situ prepared chlorophosphite was dissolved in toluene (8 $\mathrm{mL} / 2.2 \mathrm{mmol}$ BINOL) and triethylamine is added ( $5 \mathrm{mmol} / 2.2 \mathrm{mmol} \mathrm{BINOL}) .1 .0 \mathrm{Eq}$ of the desired aminoalcohol 5-10 was azeotropically dried with toluene. Then it was dissolved in toluene $(8 \mathrm{~mL} / 1.0 \mathrm{mmol}$ substrate) and triethylamine $(5.0 \mathrm{eq})$ was added. This solution was added slowly to the chlorophosphite solution at $0{ }^{\circ} \mathrm{C}$ over $5 \mathrm{~min}$ and the mixture was kept at this temperature for $5 \mathrm{~min}$. The reaction solution was then stirred at $50{ }^{\circ} \mathrm{C}$ (in an oil bath) for $16 \mathrm{~h}$. After this time, the mixture was cooled to room temperature and concentrated in vacuo. The residue was purified by column chromatography (basic silica, toluene) to give L6-L11 $\left(S_{\circ}, S_{N}\right)$ or $\left(R_{O}, R_{N}\right)$.

L6 $\left(S_{o}, S_{N}\right) .{ }^{4}$ Starting from $(S)$-BINOL (630 mg, $\left.2.2 \mathrm{mmol}\right)$, 5-cyclohexylamino-5-deoxy1,2-O-isopropylidene- $\alpha$-D-xylofuranose (5) $(272 \mathrm{mg}, 1.0 \mathrm{mmol})$ and $\mathrm{Et}_{3} \mathrm{~N}(1.01 \mathrm{~g}, 10.0$ $\mathrm{mmol})$ in toluene $(16 \mathrm{~mL})$, ligand $\mathbf{L 6}\left(S_{O}, S_{N}\right)$ was isolated as a white solid $(875 \mathrm{mg}, 97$ \%) (basic-SiO ${ }_{2}$, toluene) . [a]D $\mathrm{D}^{26}=+402.0\left(\mathrm{c}=0.75, \mathrm{CHCl}_{3}\right) .{ }^{31} \mathrm{P}\left\{{ }^{1} \mathrm{H}\right\} \mathrm{NMR}\left(\mathrm{C}_{6} \mathrm{D}_{6}, 101\right.$ $\mathrm{MHz}): \delta=163.3(\mathrm{~d}, J=3.9 \mathrm{~Hz}), 163.5(\mathrm{~d}, J=3.9 \mathrm{~Hz}) .{ }^{1} \mathrm{H}$ NMR $\left(\mathrm{C}_{6} \mathrm{D}_{6}, 250 \mathrm{MHz}\right.$ ): $\delta=$ 7.79-6.82 (m, 24H), $5.58(\mathrm{~d}, 1 \mathrm{H}, J=3.7 \mathrm{~Hz}), 4.58-4.52(\mathrm{~m}, 2 \mathrm{H}), 4.07(\mathrm{~d}, 1 \mathrm{H}, J=3.7 \mathrm{~Hz})$, $3.73(\mathrm{ddd}, 1 \mathrm{H}, J=15.4 \mathrm{~Hz}, J=6.0 \mathrm{~Hz}, J=3.5 \mathrm{~Hz}), 3.47-3.24(\mathrm{~m}, 2 \mathrm{H}), 2.24-2.11(\mathrm{~m}, 2 \mathrm{H})$, 1.93-1.77 (m, 2H), 1.72-1.55 (m, 2H), $1.32(\mathrm{~s}, 3 \mathrm{H}), 1.14-0.88\left(\mathrm{~m}, 4 \mathrm{H}, 2 \mathrm{CH}_{2}\right), 0.79(\mathrm{~s}, 3 \mathrm{H})$. ${ }^{13} \mathrm{C}\left\{{ }^{1} \mathrm{H}\right\} \operatorname{NMR}\left(\mathrm{C}_{6} \mathrm{D}_{6}, 75 \mathrm{MHz}\right): \delta=150.9-122.2,111.6,105.2,84.5(\mathrm{~d}, J=1.0 \mathrm{~Hz}), 82.6$ (m), $78.3(\mathrm{~d}, J=10.9 \mathrm{~Hz}), 58.6\left(\mathrm{~d}, J_{\mathrm{C}}=23.0 \mathrm{~Hz}\right), 44.1(\mathrm{~d}, J=6.1 \mathrm{~Hz}), 35.2(\mathrm{~d}, J=7.8$ $\mathrm{Hz}$ ), 34.2 (d, $J=9.9 \mathrm{~Hz}), 26.9,26.7,26.1,25.8$. HRMS (ESI) m/z: [M + Na]+ Calcd for $\mathrm{C}_{54} \mathrm{H}_{47} \mathrm{NO}_{8} \mathrm{P}_{2} \mathrm{Na}$ 922.2669; Found 922.2662.

L7 $\left(S_{o}, S_{N}\right) .{ }^{4}$ Starting from (S)-BINOL (630 mg, $2.2 \mathrm{mmol}$ ), 5-deoxy-1,2-O-isopropylidene5-methylamino- $\alpha$-D-xylofuranose (6) (203 mg, $1.0 \mathrm{mmol}$ ) and $\mathrm{Et}_{3} \mathrm{~N}(1.01 \mathrm{~g}, 10.0 \mathrm{mmol})$ in toluene $(16 \mathrm{~mL})$, ligand $\mathbf{L 7}\left(S_{O}, S_{N}\right)$ was isolated as a white solid $(703 \mathrm{mg}, 85 \%)$ (basic$\mathrm{SiO}_{2}$, toluene). $[\alpha] \mathrm{D}^{26}=+335.0\left(\mathrm{c}=0.53, \mathrm{CHCl}_{3}\right) .{ }^{31} \mathrm{P}\left\{{ }^{1} \mathrm{H}\right\} \mathrm{NMR}\left(\mathrm{C}_{6} \mathrm{D}_{6}, 101 \mathrm{MHz}\right): \delta=$ 155.8 (d, $J=4.0 \mathrm{~Hz}), 163.8$ (d, J = 4.0 Hz). ${ }^{1} \mathrm{H}$ NMR ( $\left.\mathrm{C}_{6} \mathrm{D}_{6}, 250 \mathrm{MHz}\right): \delta=7.74-6.83$ (m, $24 \mathrm{H}), 5.76(\mathrm{~d}, 1 \mathrm{H}, J=3.7 \mathrm{~Hz}), 4.64(\mathrm{dd}, 1 \mathrm{H}, J=9.3 \mathrm{~Hz}, J=2.6 \mathrm{~Hz}), 4.49-4.43(\mathrm{~m}, 1 \mathrm{H})$, $4.31(\mathrm{~d}, 1 \mathrm{H}, J=3.7 \mathrm{~Hz}$ ) , 3.82 (ddd, $1 \mathrm{H}, J=9.9 \mathrm{~Hz}, J=3.5 \mathrm{~Hz}$ ), 3.41 (ddd, $1 \mathrm{H}, J=19.2$ $\mathrm{Hz}, J=14.7 \mathrm{~Hz}, J=8.1 \mathrm{~Hz}), 2.48(\mathrm{~d}, 3 \mathrm{H}, J=5.5 \mathrm{~Hz}), 1.36(\mathrm{~s}, 3 \mathrm{H}), 0.97(\mathrm{~s}, 3 \mathrm{H}) \cdot{ }^{13} \mathrm{C}\left\{{ }^{1} \mathrm{H}\right\}$ $\operatorname{NMR}\left(\mathrm{C}_{6} \mathrm{D}_{6}, 75 \mathrm{MHz}\right): \delta=150.8-121.9,111.8,105.5,84.7,80.8(\mathrm{~m}), 78.4(\mathrm{~d}, J=5.9 \mathrm{~Hz})$, 
49.5 (d, $J=36.2 \mathrm{~Hz}$ ), 33.9 (d, $J=3.9 \mathrm{~Hz}$ ), 26.9, 26.2. HRMS (ESI) m/z: [M + Na]+ Calcd for $\mathrm{C}_{49} \mathrm{H}_{39} \mathrm{NO}_{8} \mathrm{P}_{2} \mathrm{Na}$ 854.2043; Found 854.2040.

L8 $\left(S_{o}, S_{N}\right) .{ }^{4}$ Starting from $(S)$-BINOL (630 mg, 2.2 mmol), 5-benzylamino-5-deoxy-1,2O-isopropylidene- $\alpha$-D-xylofuranose (7) $\left(279 \mathrm{mg}, 1.0 \mathrm{mmol}\right.$ ) and $\mathrm{Et}_{3} \mathrm{~N}(1.01 \mathrm{~g}, 10.0 \mathrm{mmol})$ in toluene $(16 \mathrm{~mL})$, ligand $\mathbf{L 8}\left(S_{\circ}, S_{N}\right)$ was isolated as a white solid $(731 \mathrm{mg}, 81 \%)$ (basic$\mathrm{SiO}_{2}$, toluene). $[\alpha] \mathrm{D}^{27}=+319.2\left(\mathrm{c}=0.80, \mathrm{CHCl}_{3}\right) \cdot{ }^{31} \mathrm{P}\left\{{ }^{1} \mathrm{H}\right\} \mathrm{NMR}\left(\mathrm{C}_{6} \mathrm{D}_{6}, 101 \mathrm{MHz}\right): \delta=$ 161.7 (s), 161.8 (s). ${ }^{1} \mathrm{H}$ NMR $\left(\mathrm{C}_{6} \mathrm{D}_{6}, 250 \mathrm{MHz}\right): \delta=7.74-6.83(\mathrm{~m}, 29 \mathrm{H}), 5.69(\mathrm{~d}, 1 \mathrm{H}, J=$ 3.8 Hz), 4.69-4.64 (m, 1H), 4.61-4.53 (dd, $1 \mathrm{H}, J=14.9 \mathrm{~Hz}, J=6.3 \mathrm{~Hz}), 4.45(\mathrm{dd}, 1 \mathrm{H}, J=$ $9.4 \mathrm{~Hz}, J=2.6 \mathrm{~Hz}$ ), 4.30 (dd, $1 \mathrm{H}, J=14.9 \mathrm{~Hz}, J=10.4 \mathrm{~Hz}), 4.23(\mathrm{~d}, 1 \mathrm{H}, J=3.8 \mathrm{~Hz}), 3.61$ (ddd, $1 \mathrm{H}, J=15.1 \mathrm{~Hz}$ ), 3.39 (ddd, $1 \mathrm{H}, J=19.3 \mathrm{~Hz}, J=15.1 \mathrm{~Hz}, J=8.3 \mathrm{~Hz}$ ), 1.40 (s, 3H), $0.93(\mathrm{~s}, 3 \mathrm{H}) .{ }^{13} \mathrm{C}\left\{{ }^{1} \mathrm{H}\right\} \mathrm{NMR}\left(\mathrm{C}_{6} \mathrm{D}_{6}, 63 \mathrm{MHz}\right.$ ) : $\delta(\mathrm{ppm})=150.6-122.1,111.8,105.7,84.4(\mathrm{~d}$, $J=1.3 \mathrm{~Hz}), 82.9(\mathrm{~m}), 78.9(\mathrm{~d}, J=13.6 \mathrm{~Hz}), 50.3(\mathrm{~d}, J=14.1 \mathrm{~Hz}), 45.0(\mathrm{~d}, J=23.2 \mathrm{~Hz})$, 26.9, 26.1. HRMS (ESI) $\mathrm{m} / \mathrm{z}$ : $[\mathrm{M}+\mathrm{Na}]+$ Calcd for $\mathrm{C}_{55} \mathrm{H}_{43} \mathrm{NO}_{8} \mathrm{P}_{2} \mathrm{Na} 930.2356$; Found 930.2340 .

L9 $\left(S_{o}, S_{N}\right){ }^{4}$ Starting from (S)-BINOL (630 mg, $2.2 \mathrm{mmol}$ ), 5-deoxy-5-isopropylamino1,2-O-isopropylidene-a-D-xylofuranose (8) $\left(231 \mathrm{mg}, 1.0 \mathrm{mmol}\right.$ ) and $\mathrm{Et}_{3} \mathrm{~N}(1.01 \mathrm{~g}, 10.0$ $\mathrm{mmol}$ ) in toluene $(16 \mathrm{~mL})$, ligand $\mathbf{L 9}\left(S_{O}, S_{N}\right)$ was isolated as a white solid (653 $\mathrm{mg}, 76$ \%) (basic-SiO 2 , toluene). [a]D ${ }^{25}=+427.9\left(\mathrm{c}=0.58, \mathrm{CHCl}_{3}\right) .{ }^{31} \mathrm{P}\left\{{ }^{1} \mathrm{H}\right\} \mathrm{NMR}\left(\mathrm{C}_{6} \mathrm{D}_{6}, 101\right.$ $\mathrm{MHz}): \delta=162.9$ (d, $J=5.0 \mathrm{~Hz}), 163.5$ (d, $J=5.0 \mathrm{~Hz}) .{ }^{1} \mathrm{H}$ NMR $\left(\mathrm{C}_{6} \mathrm{D}_{6}, 250 \mathrm{MHz}\right): \delta=$ 7.76-6.80 (m, 24H), $5.57(\mathrm{~d}, 1 \mathrm{H}, J=3.7 \mathrm{~Hz}), 4.57-4.49(\mathrm{~m}, 2 \mathrm{H}), 4.08(\mathrm{~d}, 1 \mathrm{H}, J=3.7 \mathrm{~Hz})$, $3.85(\mathrm{~m}, 1 \mathrm{H}$ ), 3.70 (ddd, $1 \mathrm{H}, J=15.5 \mathrm{~Hz}, J=6.1 \mathrm{~Hz}, J=3.5 \mathrm{~Hz}$ ), 3.27 (ddd, $1 \mathrm{H}, J=15.5$ $\mathrm{Hz}, J=9.4 \mathrm{~Hz}, J=6.3 \mathrm{~Hz}), 1.33(\mathrm{~d}, 3 \mathrm{H}, J=6.8 \mathrm{~Hz}), 1.32$ (d, $3 \mathrm{H}, J=6.8 \mathrm{~Hz}), 1.30$ (s, $3 \mathrm{H}), 0.80(\mathrm{~s}, 3 \mathrm{H}) .{ }^{13} \mathrm{C}\left\{{ }^{1} \mathrm{H}\right\}$ NMR $\left(\mathrm{C}_{6} \mathrm{D}_{6}, 63 \mathrm{MHz}\right): \delta=150.8-122.2,111.6,105.2,84.5$ (d, $J=1.5 \mathrm{~Hz}$ ), $82.4(\mathrm{~m}), 78.3(\mathrm{~d}, J=10.8 \mathrm{~Hz}), 49.3(\mathrm{~d}, J=25.9 \mathrm{~Hz}), 43.1$ (d, $J=8.1 \mathrm{~Hz})$, 26.8, 26.1, 23.6 (d, $J=7.0 \mathrm{~Hz}$ ), 23.0 (d, $J=9.6 \mathrm{~Hz}$ ). HRMS (ESI) m/z: [M + Na]+ Calcd for $\mathrm{C}_{51} \mathrm{H}_{43} \mathrm{NO}_{8} \mathrm{P}_{2} \mathrm{Na}$ 882.2356; Found 823.2345.

L10 $\left(S_{o}, S_{N}\right) .{ }^{4}$ Starting from $(S)$-BINOL $(630 \mathrm{mg}, 2.2 \mathrm{mmol}), \quad$ 5-deoxy-1,2-O-

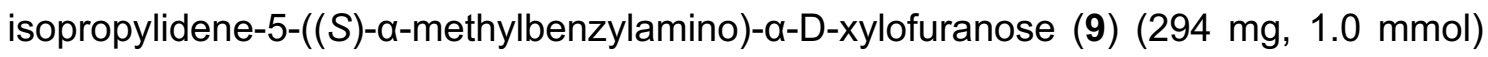
and $\mathrm{Et}_{3} \mathrm{~N}(1.01 \mathrm{~g}, 10.0 \mathrm{mmol})$ in toluene $(16 \mathrm{~mL})$, ligand $\mathbf{L} 10\left(S_{\circ}, S_{N}\right)$ was isolated as a white solid (824 mg, $89 \%$ ) (basic-SiO 2 , toluene). [a]D $\mathrm{D}^{23}=+313.8$ (c=1.00, $\mathrm{CHCl}_{3}$ ). ${ }^{31} \mathrm{P}\left\{{ }^{1} \mathrm{H}\right\} \operatorname{NMR}\left(\mathrm{C}_{6} \mathrm{D}_{6}, 121 \mathrm{MHz}\right): \delta=159.7$ (s), $165.1(\mathrm{~s}) .{ }^{1} \mathrm{H}$ NMR $\left(\mathrm{C}_{6} \mathrm{D}_{6}, 300 \mathrm{MHz}\right): \delta=$ 7.77-6.80 (m, 29H), $5.50(\mathrm{~d}, 1 \mathrm{H}, J=3.8 \mathrm{~Hz}), 5.06(\mathrm{dq}, 1 \mathrm{H}, J=14.2 \mathrm{~Hz}, J=7.1 \mathrm{~Hz}), 4.56-$ $4.52(\mathrm{~m}, 1 \mathrm{H}), 4.27(\mathrm{dd}, 1 \mathrm{H}, J=8.9 \mathrm{~Hz}, J=2.6 \mathrm{~Hz}), 3.98(\mathrm{~d}, 1 \mathrm{H}, J=3.8 \mathrm{~Hz}), 3.52$ (ddd, $1 \mathrm{H}, J=15.2 \mathrm{~Hz}, J=2.9 \mathrm{~Hz}, J=2.9 \mathrm{~Hz}), 3.02(\mathrm{ddd}, 1 \mathrm{H}, J=15.2 \mathrm{~Hz}, J=7.2 \mathrm{~Hz}, J=2.8$ 
$\mathrm{Hz}$ ), 1.80 (dd, $3 \mathrm{H}, J=7.1 \mathrm{~Hz}, J=3.8 \mathrm{~Hz}), 1.26(\mathrm{~s}, 3 \mathrm{H}), 0.76(\mathrm{~s}, 3 \mathrm{H}) .{ }^{13} \mathrm{C}\left\{{ }^{1} \mathrm{H}\right\} \mathrm{NMR}\left(\mathrm{C}_{6} \mathrm{D}_{6}\right.$, $75 \mathrm{MHz}$ ): $\delta$ = 151.0-122.0, 111.5, 105.5, 84.1, $83.0(\mathrm{~m}), 78.8$ (d, J3 = 15.8 Hz), 59.6 (d, $J=23.7 \mathrm{~Hz}), 45.5,26.8,25.9,23.2(\mathrm{~d}, J=30.4 \mathrm{~Hz})$. HRMS (ESI) m/z: [M + Na]+ Calcd for $\mathrm{C}_{56} \mathrm{H}_{45} \mathrm{NO}_{8} \mathrm{P}_{2} \mathrm{Na}$ 944.2513; Found 944.2514.

L10 $\left(\boldsymbol{R}_{O}, \boldsymbol{R}_{\boldsymbol{N}}\right){ }^{4}$ Starting from $(R)$-BINOL $(630 \mathrm{mg}, 2.2 \mathrm{mmol}), \quad$ 5-deoxy-1,2-O-

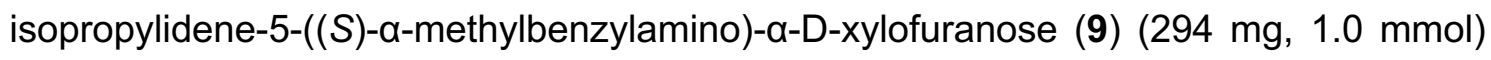
and $\mathrm{Et}_{3} \mathrm{~N}(1.01 \mathrm{~g}, 10.0 \mathrm{mmol})$ in toluene $(16 \mathrm{~mL})$, ligand $\mathbf{L} 10\left(R_{O}, R_{N}\right)$ was isolated as a white solid (570 mg, $62 \%$ ) (basic-SiO ${ }_{2}$, toluene). [a]D ${ }^{24}=-386.4$ (c = 0.73, $\mathrm{CHCl}_{3}$ ). ${ }^{31} \mathrm{P}\left\{{ }^{1} \mathrm{H}\right\} \operatorname{NMR}\left(\mathrm{C}_{6} \mathrm{D}_{6}, 101 \mathrm{MHz}\right): \delta=158.6(\mathrm{~d}, J=17.1 \mathrm{~Hz}), 161.2(\mathrm{~d}, J=17.1 \mathrm{~Hz}) .{ }^{1} \mathrm{H}$ $\operatorname{NMR}\left(\mathrm{C}_{6} \mathrm{D}_{6}, 250 \mathrm{MHz}\right): \delta=7.81-6.78(\mathrm{~m}, 29 \mathrm{H}), 5.77(\mathrm{~d}, 1 \mathrm{H}, J=3.7 \mathrm{~Hz}), 5.02-4.90(\mathrm{~m}$, $1 \mathrm{H}), 4.55(\mathrm{~d}, 1 \mathrm{H}, J=3.7 \mathrm{~Hz}), 4.30-4.34(\mathrm{~m}, 2 \mathrm{H}), 3.76-3.64(\mathrm{ddd}, 1 \mathrm{H}, J=15.1 \mathrm{~Hz}, J=$ $11.3 \mathrm{~Hz}, J=3.4 \mathrm{~Hz}), 3.51-3.39(\mathrm{~m}, 1 \mathrm{H}), 1.53(\mathrm{~d}, 3 \mathrm{H}, J=7.2 \mathrm{~Hz}), 1.36(\mathrm{~s}, 3 \mathrm{H}), 1.04$ (s, $3 \mathrm{H}) .{ }^{13} \mathrm{C}\left\{{ }^{1} \mathrm{H}\right\} \operatorname{NMR}\left(\mathrm{C}_{6} \mathrm{D}_{6}, 63 \mathrm{MHz}\right): \delta=150.3-121.7,111.8,105.2,85.0(\mathrm{~d}, J=2.8 \mathrm{~Hz})$, $80.7(\mathrm{~m}), 78.6$ (d, $J=11.9 \mathrm{~Hz}), 54.7$ (d, $J=14.0 \mathrm{~Hz}), 43.6$ (d, $J=18.9 \mathrm{~Hz}), 27.2,26.4$, 20.7 (d, $J=12.1 \mathrm{~Hz}$ HRMS (ESI) m/z: [M + Na]+ Calcd for $\mathrm{C}_{56} \mathrm{H}_{45} \mathrm{NO}_{8} \mathrm{P}_{2} \mathrm{Na}$ 944.2513; Found 944.2503.

L11 $\left(S_{o}, S_{N}\right) .{ }^{4}$ Starting from $(S)$-BINOL $(630 \mathrm{mg}, 2.2 \mathrm{mmol})$, 5-tert-butylamino-5-deoxy1,2-O-isopropylidene-a-D-xylofuranose (10) (245 mg,1.0 mmol) and $\mathrm{Et}_{3} \mathrm{~N}(1.01 \mathrm{~g}, 10.0$ $\mathrm{mmol})$ in toluene $(16 \mathrm{~mL})$, ligand $\mathbf{L} 11\left(S_{O}, S_{N}\right)$ was isolated as a white solid $(250 \mathrm{mg}, 29$ \%) (basic-SiO 2 , toluene). ${ }^{31} \mathrm{P}\left\{{ }^{1} \mathrm{H}\right\} \mathrm{NMR}\left(\mathrm{CDCl}_{3}, 101 \mathrm{MHz}\right): \delta=150.6(\mathrm{~s}), 151.9(\mathrm{~s}) .{ }^{1} \mathrm{H}$ $\operatorname{NMR}\left(\mathrm{CDCl}_{3}, 250 \mathrm{MHz}\right): \delta=7.75-6.69(\mathrm{~m}, 24 \mathrm{H}), 5.43(\mathrm{~d}, 1 \mathrm{H}, J=3.7 \mathrm{~Hz}), 4.57-4.54(\mathrm{~m}$, $2 \mathrm{H}$ ), 3.88 (d, $1 \mathrm{H}, J=3.7 \mathrm{~Hz}$ ), 3.78 (ddd, $1 \mathrm{H}, J=16.0 \mathrm{~Hz}$ ), 3.26 (ddd, $1 \mathrm{H}, J=16.1 \mathrm{~Hz}$ ), $1.62(\mathrm{~d}, 9 \mathrm{H}, J=2.5 \mathrm{~Hz}), 1.31(\mathrm{~s}, 3 \mathrm{H}), 0.70(\mathrm{~s}, 3 \mathrm{H}) .{ }^{13} \mathrm{C}\left\{{ }^{1} \mathrm{H}\right\} \operatorname{NMR}\left(\mathrm{C}_{6} \mathrm{D}_{6}, 63 \mathrm{MHz}\right): \delta=$ 151.3-122.0, 111.4, 104.9, 84.3, 84.2, 78.6 (d, $J=12.5 \mathrm{~Hz}), 56.2$ (d, $J=22.0 \mathrm{~Hz}), 42.7$ (d, $J=5.8 \mathrm{~Hz}$ ), 31.6 (d, $J=15.8 \mathrm{~Hz}), 26.9,25.9$. HRMS (ESI) m/z: [M + Na]+ Calcd for $\mathrm{C}_{52} \mathrm{H}_{45} \mathrm{NO}_{8} \mathrm{P}_{2} \mathrm{Na}$ 896.2513; Found 896.2494.

\section{General procedure for the preparation of phosphite-phosphoramidite ligands L10 $\left(S_{N}, R_{O}\right.$ and $\left.R_{O}, S_{N}\right)$.}

1.1 Eq of enantiopure $(R)$ - or (S)-BINOL were suspended in phosphorus trichloride (1.5 $\mathrm{mL} / 1.0 \mathrm{mmol}$ BINOL), 2-3 drops of N-methyl-2-pyrrolidone are added and the solution was heated in an oil bath to $75^{\circ} \mathrm{C}$ for $5 \mathrm{~min}$. The resulting $\mathrm{HCl}$ gas was derived from the reaction vessel by using a bubble counter (slight argon stream!). The now clear solution was cooled to room temperature, concentrated and dried azeotropically with toluene 
(three times). Thus, the in situ prepared chlorophosphite was dissolved in toluene (5 $\mathrm{mL} / 1.1 \mathrm{mmol}$ BINOL) and pyridine (2.3 eq) was added. $1.0 \mathrm{Eq}$ of azeotropically dried amino sugar 9 was dissolved in toluene ( $5 \mathrm{~mL} / 1.0 \mathrm{mmol}$ substrate) and pyridine (2.3 eq) was added. The chlorophosphite solution was added slowly to the sugar solution at $0{ }^{\circ} \mathrm{C}$ over $5 \mathrm{~min}$ and the mixture was kept at this temperature for $5 \mathrm{~min}$. The reaction solution was warmed in an oil bath to $80{ }^{\circ} \mathrm{C}$ and stirred for $16 \mathrm{~h}$. After this time, the mixture was cooled to room temperature and concentrated in vacuo. Because the residue couldn't be purified by column chromatography or recrystallization, crude products $11\left(R_{0}\right)$ or 12 $\left(S_{0}\right)$ were used in the next step without further purification. 1.1 Eq of the corresponding chlorophosphite of the aromatic diol were dissolved in toluene $(10 \mathrm{~mL} / 1.1 \mathrm{mmol}$ chlorophosphite) and triethylamine (2.0 eq) is added. $1.0 \mathrm{Eq}$ of the crude $11\left(R_{0}\right)$ or 12 (So) were dissolved in toluene $(10 \mathrm{~mL} / 1.0 \mathrm{mmol}$ substrate) and triethylamine (2.0 eq) was added. The chlorophosphite solution was added slowly to the sugar solution at $0{ }^{\circ} \mathrm{C}$ over $5 \mathrm{~min}$ and the mixture was kept at this temperature for $5 \mathrm{~min}$. The reaction solution was warmed to $50^{\circ} \mathrm{C}$ and stirred for $16 \mathrm{~h}$. After this time, the mixture was cooled to room temperature and concentrated in vacuo. The residue was purified by column chromatography (basic silica, toluene) to give ligands $\operatorname{L10}\left(\boldsymbol{S}_{\boldsymbol{N}}, \boldsymbol{R}_{\mathbf{O}}\right)$ and $\left(\boldsymbol{R}_{\boldsymbol{O}}, \boldsymbol{S}_{\boldsymbol{N}}\right)$.

L10 $\left(\boldsymbol{R}_{\mathbf{O}}, \boldsymbol{S}_{\boldsymbol{N}}\right) \cdot{ }^{4}$ Starting from $(R)$-BINOL (158 mg, $0.55 \mathrm{mmol}$ ), (S)-BINOL (158 mg, 0.55 mmol), 5-deoxy-1,2-O-isopropylidene-5-((S)-a-methylbenzylamino)- $\alpha-D-x y l o f u r a n o s e$ (9) $(147 \mathrm{mg}, 0.5 \mathrm{mmol})$, pyridine (182 mg, $2.3 \mathrm{mmol}$ ) and $\mathrm{Et}_{3} \mathrm{~N}$ (202 mg, $\left.2.0 \mathrm{mmol}\right)$ in toluene $(5 \mathrm{~mL})$, ligand L10 $\left(R_{0}, S_{N}\right)$ was isolated as a white solid (97 mg, $21 \%$ ) (basic$\mathrm{SiO}_{2}$, toluene). [ $\left.\alpha\right] \mathrm{D}^{26}=-49.6\left(\mathrm{c}=0.28, \mathrm{CHCl}_{3}\right) \cdot{ }^{31} \mathrm{P}\left\{{ }^{1} \mathrm{H}\right\} \mathrm{NMR}\left(\mathrm{C}_{6} \mathrm{D}_{6}, 121 \mathrm{MHz},\right): \delta=148.1$ (s), 146.5 (s). ${ }^{1} \mathrm{H}$ NMR ( $\mathrm{C}_{6} \mathrm{D}_{6}, 300 \mathrm{MHz}$ ): $\delta=7.83-7.18(\mathrm{~m}, 2 \mathrm{H}), 6.60-7.14(\mathrm{~m}, 9 \mathrm{H}), 5.79$ $(\mathrm{d}, 1 \mathrm{H}, J=3.7 \mathrm{~Hz}), 5.20-5.07(\mathrm{~m}, 1 \mathrm{H}), 4.53-4.49(\mathrm{~m}, 2 \mathrm{H}), 4.31(\mathrm{dd}, 1 \mathrm{H}, J=9.8 \mathrm{~Hz}, J=$ $2.8 \mathrm{~Hz}$ ), $3.74(\mathrm{~m}, 1 \mathrm{H}), 3.08$ (ddd, $1 \mathrm{H}, J=15.2 \mathrm{~Hz}, J=7.7 \mathrm{~Hz}, J=2.2 \mathrm{~Hz}), 1.88(\mathrm{dd}, 3 \mathrm{H}$, $J=7.2 \mathrm{~Hz}, J=3.2 \mathrm{~Hz}), 1.36(\mathrm{~s}, 3 \mathrm{H}), 1.06(\mathrm{~s}, 3 \mathrm{H}) \cdot{ }^{13} \mathrm{C}\left\{{ }^{1} \mathrm{H}\right\} \operatorname{NMR}\left(\mathrm{C}_{6} \mathrm{D}_{6}, 63 \mathrm{MHz}\right): \delta=$ 121.8-151.2, 111.7, 105.2, 84.4 (d, $J=1.6 \mathrm{~Hz}), 83.3(\mathrm{~m}), 79.8,59.2(\mathrm{~d}, J=22.4 \mathrm{~Hz})$, 45.7, 30.2, 26.7, 26.1. HRMS (ESI) m/z: [M + Na]+ Calcd for $\mathrm{C}_{56} \mathrm{H}_{45} \mathrm{NO}_{8} \mathrm{P}_{2} \mathrm{Na} 944.2513$; Found 944.2506 .

L10 $\left(\boldsymbol{S}_{o}, \boldsymbol{R}_{\boldsymbol{N}}\right) .{ }^{4}$ Starting from (S)-BINOL (158 mg, $\left.0.55 \mathrm{mmol}\right),(R)-B I N O L$ (158 mg, 0.55 mmol), 5-deoxy-1,2-O-isopropylidene-5-((S)-a-methylbenzylamino)- $\alpha-D-x y l o f u r a n o s e$ (9) $(147 \mathrm{mg}, 0.5 \mathrm{mmol})$, pyridine (182 $\mathrm{mg}, 2.3 \mathrm{mmol})$ and Et3N (202 mg, $2.0 \mathrm{mmol})$ in toluene, the product L10 $\left(S_{O}, R_{N}\right)$ was isolated as a white solid $(161 \mathrm{mg}, 35 \%)$ (basic-

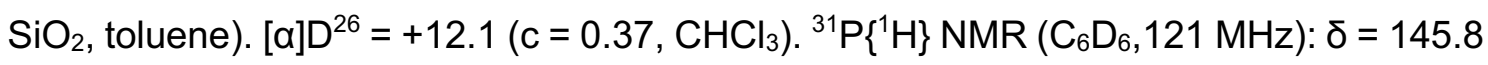
(s), 133.1 (s). ${ }^{1} \mathrm{H}$ NMR $\left(\mathrm{C}_{6} \mathrm{D}_{6}, 300 \mathrm{MHz},\right): \delta=7.80-7.19(\mathrm{~m}, 20 \mathrm{H}), 7.16-7.00(\mathrm{~m}, 5 \mathrm{H})$, 
6.91-6.81 (m, 4H), $5.60(\mathrm{~d}, 1 \mathrm{H}, J=3.7 \mathrm{~Hz}$ ), $4.98(\mathrm{~m}, 1 \mathrm{H}), 4.40-4.36(\mathrm{~m}, 1 \mathrm{H}), 4.26-4.22$ $(\mathrm{m}, 2 \mathrm{H}), 3.75(\mathrm{ddd}, 1 \mathrm{H}, J=15.5 \mathrm{~Hz}, J=9.1 \mathrm{~Hz}, J=2.8 \mathrm{~Hz}), 3.42-3.32(\mathrm{~m}, 1 \mathrm{H}), 1.62(\mathrm{~d}$, $3 \mathrm{H}, J=6.7 \mathrm{~Hz}), 1.40(\mathrm{~s}, 3 \mathrm{H}), 0.96(\mathrm{~s}, 3 \mathrm{H}) .{ }^{13} \mathrm{C}\left\{{ }^{1} \mathrm{H}\right\} \mathrm{NMR}\left(\mathrm{C}_{6} \mathrm{D}_{6}, 63 \mathrm{MHz}\right): \delta=150.3-121.9$, 111.5, 105.4, 84.7 (d, $J=2.2 \mathrm{~Hz}$ ), $81.0(\mathrm{~m}), 78.8$ (d, $J=13.2 \mathrm{~Hz}), 55.6,42.6$ (d, $J=10.9$ $\mathrm{Hz}), 27.1,26.5,21.5(\mathrm{~d}, J=15.7 \mathrm{~Hz})$. HRMS (ESI) m/z: [M + Na]+ Calcd for $\mathrm{C}_{56} \mathrm{H}_{45} \mathrm{NO}_{8} \mathrm{P}_{2} \mathrm{Na}$ 944.2513; Found 944.2511.

\section{SIII. Synthesis of $\alpha$-alkyl acrylate acids}

General Procedure A: Synthesis of a-alkyl acrylate acids 11.

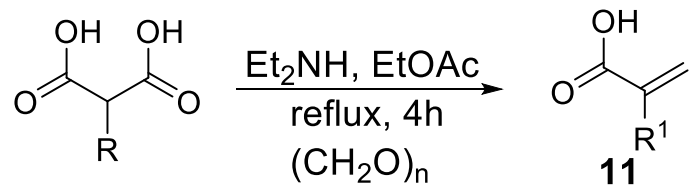

According to a modified literature procedure, ${ }^{5}$ the diacid (1 eq.) was dissolved in EtOAc $(0.75 \mathrm{M})$ and the resulting solution was cooled to $0^{\circ} \mathrm{C}$ in an ice bath, diethylamine $(1.01$ eq.) was added dropwise and subsequent addition of paraformaldehyde (1.5 eq.). The resulting suspension was refluxed for 2 hours in an oil bath and then the reaction mixture was cooled to $0^{\circ} \mathrm{C}$, diluted with $\mathrm{H}_{2} \mathrm{O}(0.6 \mathrm{~mL} / \mathrm{mmol}$ diacid $)$ and acidified to $\mathrm{pH} 1$ with concentrated $\mathrm{HCl}$. The resulting mixture was extracted with EtOAc $(3 x)$ and the combined organics phases were washed with saturated brine, dried over anhydrous $\mathrm{MgSO}_{4}$ and concentrated under reduced pressure. The crude was purified by flash chromatography on silica gel eluting with PE/EtOAc to afford the corresponding a-alkyl acrylate acid 11 .

\section{3-methyl-2-methylenebutanoic acid (11a). ${ }^{5}$}<smiles>C=C(C(=O)O)C(C)C</smiles>

General procedure A was followed by employing iso-propylmalonic acid (4.38 $\mathrm{g}, 30 \mathrm{mmol})$. Purification by flash chromatography eluting with PE/EtOAc (1:1) afforded $11 \mathrm{a}(3.0 \mathrm{~g}, 88 \%)$ as colourless oil. ${ }^{1} \mathrm{H} \mathrm{NMR}\left(\mathrm{CDCl}_{3}, 400 \mathrm{MHz}\right)$ $\delta: 6.29(\mathrm{~s}, 1 \mathrm{H}), 5.65\left(\mathrm{t}, 1 \mathrm{H}, J_{\mathrm{H}, \mathrm{H}}=1.1 \mathrm{~Hz}\right), 2.81$ (septd, $1 \mathrm{H}, J=1.0 \mathrm{~Hz}, J_{\mathrm{H}, \mathrm{H}}=6.8 \mathrm{~Hz}$ ), $1.11(\mathrm{~d}, 6 \mathrm{H}, J=6.8 \mathrm{~Hz}) \cdot{ }^{13} \mathrm{C}\left\{{ }^{1} \mathrm{H}\right\} \mathrm{NMR}\left(\mathrm{CDCl}_{3}, 100.6 \mathrm{MHz}\right) \delta: 173.0,146.6,124.4,29.2$, 21.9. These signals are in agreement with those reported in the literature.

2-methylenebutanoic acid (11b). ${ }^{5}$ 
(-)

General procedure A was followed by employing 2-ethylmalonic acid (3.15 g, $23.8 \mathrm{mmol})$. Purification by flash chromatography eluting with PE/EtOAc (7:3) afforded $11 \mathrm{~b}(2.04 \mathrm{~g}, 86 \%)$ as colourless oil. ${ }^{1} \mathrm{H}$ NMR $\left(\mathrm{CDCl}_{3}, 400 \mathrm{MHz}\right) \delta$ : 11.85 (bs, 1H), 6.30 (q, 1H, J = $1.1 \mathrm{~Hz}$ ), 5.66 (q, 1H, $J=1.4 \mathrm{~Hz}$ ), 2.34 (qq, 2H, $J=0.9$ $\left.\mathrm{Hz}, J_{\mathrm{H}, \mathrm{H}}=7.4 \mathrm{~Hz}\right), 1.10(\mathrm{t}, 3 \mathrm{H}, J=7.4 \mathrm{~Hz}) \cdot{ }^{13} \mathrm{C}\left\{{ }^{1} \mathrm{H}\right\} \mathrm{NMR}\left(\mathrm{CDCl}_{3}, 100.6 \mathrm{MHz}\right) \delta: 173.2$, $141.8,126.1,24.6,12.8$. These signals are in agreement with those reported in the literature.

\section{2-cyclopentylacrylic acid (11c). ${ }^{5}$}

$\mathrm{OH} \quad$ General procedure A was followed by employing cyclopentylmalonic acid (3.0 g, $17.4 \mathrm{mmol})$. Purification by flash chromatography eluting with PE/EtOAc

(8:2) afforded $11 \mathrm{c}(2.27 \mathrm{~g}, 93 \%)$ as colourless oil. ${ }^{1} \mathrm{H} \mathrm{NMR}\left(\mathrm{CDCl}_{3}, 400 \mathrm{MHz}\right)$ $\delta: 11.47(\mathrm{bs}, 1 \mathrm{H}), 6.28\left(\mathrm{t}, 1 \mathrm{H}, J_{\mathrm{H}, \mathrm{H}}=0.8 \mathrm{~Hz}\right), 5.66(\mathrm{t}, 1 \mathrm{H}, J=1.3 \mathrm{~Hz}), 2.85$ (quin, $1 \mathrm{H}, J=8.2 \mathrm{~Hz}), 1.93(\mathrm{~m}, 2 \mathrm{H}), 1.66(\mathrm{~m}, 4 \mathrm{H}), 1.42(\mathrm{~m}, 2 \mathrm{H}) .{ }^{13} \mathrm{C}\left\{{ }^{1} \mathrm{H}\right\} \mathrm{NMR}\left(\mathrm{CDCl}_{3}\right.$, 100.6 MHz) $\delta: 173.5,144.0,124.4,41.2,32.0,25.0$. These signals are in agreement with those reported in the literature.

\section{SIV. Synthesis of $\alpha$-alkyl acrylamides 1}

General Procedure B: Synthesis of a-alkyl acrylamides 1b-c.<smiles>[R]C(=C)C(=O)O</smiles>

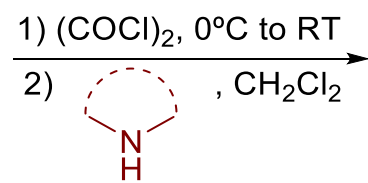<smiles>[R]C(=C)C(=O)N1CCCC1</smiles>

According to the modified literature procedure,${ }^{6}$ in a solution under argon of compound 6 (1 eq.) and N,N-Dimethylformamide (1.5 eq.) in dichloromethane (0.5 M) was slowly added at $0^{\circ} \mathrm{C}$ the oxalyl chloride (0.98 eq.). The reaction was allowed to stir at room temperature for $4 \mathrm{~h}$. Separately, under argon was prepared a solution of the amine (2.25 eq.) in dichloromethane $(0.5 \mathrm{M})$. The solution of the acyl chloride was added to the solution of the amine and the reaction was stirred at room temperature and followed by TLC until it completed. The reaction mixture was washed with $2 \mathrm{M} \mathrm{NaOH} \mathrm{(3x),} \mathrm{brine} \mathrm{(3x),}$ $1 \mathrm{M} \mathrm{HCl}(3 \mathrm{x})$ and brine $(3 \mathrm{x})$. The organic phase was dried over dried over anhydrous 
$\mathrm{MgSO}_{4}$ and concentrated under reduced pressure. The crude was purified by flash chromatography (PE/EtOAc) to afford the product 1 with moderate to high yield.

\section{$\mathrm{N}, \mathrm{N}$-diphenylmethacrylamide (1b). ${ }^{7}$}<smiles>C=C(C)C(=O)N(c1ccccc1)c1ccccc1</smiles>

General procedure B was followed by employing methacrylic acid $(2.15 \mathrm{~g}, 25 \mathrm{mmol})$ and diphenylamine $(9.12 \mathrm{~g}, 56.3 \mathrm{mmol})$. Purification by flash chromatography on silica gel eluting with PE/EtOAc (10:1) afforded $1 \mathrm{~b}(2.97 \mathrm{~g}, 50 \%)$ white solid. ${ }^{1} \mathrm{H} \mathrm{NMR}\left(\mathrm{CDCl}_{3}, 400 \mathrm{MHz}\right) \delta$ : $7.31-7.36(\mathrm{~m}, 4 \mathrm{H}), 7.20-7.25(\mathrm{~m}, 2 \mathrm{H}), 7.15-7.19(\mathrm{~m}, 4 \mathrm{H}), 5.24(\mathrm{~m}, 1 \mathrm{H}), 5.17(\mathrm{~m}$, $1 \mathrm{H}), 1.84(\mathrm{~m}, 3 \mathrm{H}) .{ }^{13} \mathrm{C}\left\{{ }^{1} \mathrm{H}\right\} \mathrm{NMR}\left(\mathrm{CDCl}_{3}, 100.6 \mathrm{MHz}\right) \delta: 172.0,143.6,141.3,129.2,127.3$, 126.6, 121.1, 20.0. These signals are in agreement with those reported in the literature.

\section{$\mathrm{N}, \mathrm{N}$-diisopropylmethacrylamide (1c). ${ }^{6}$}<smiles>C=C(C)C(=O)N(C(C)C)C(C)C</smiles>

General procedure B was followed by employing methacrylic acid $(2.15 \mathrm{~g}$, $25 \mathrm{mmol}$ ) and diisoproylamine (5.69 g, $56.3 \mathrm{mmol})$. Purification by flash chromatography on silica gel eluting with PE/EtOAc (7:3) afforded 1c (3.39 g, 80\%) as white solid. ${ }^{1} \mathrm{H}$ NMR $\left(\mathrm{CDCl}_{3}, 400 \mathrm{MHz}\right) \delta: 4.99(\mathrm{~m}, 1 \mathrm{H}), 4.91(\mathrm{~m}$, $1 \mathrm{H}$ ), 4.07 (bs, 1H), 3.41 (bs, $1 \mathrm{H}$ ), 1.92 (t, 3H, $J=1.2$ ), 1.30 (bs, $12 \mathrm{H}) .{ }^{13} \mathrm{C}\left\{{ }^{1} \mathrm{H}\right\} \mathrm{NMR}$ $\left(\mathrm{CDCl}_{3}, 100.6 \mathrm{MHz}\right) \delta: 172.2,143.1,112.6,50.3,45.5,20.8,20.6$. These signals are in agreement with those reported in the literature.

\section{$\mathrm{N}, \mathrm{N}$-diethyl-2-methylenebutanamide (1d). ${ }^{8}$}

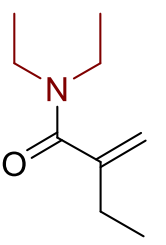

General procedure B was followed by employing 2-methylenebutanoic acid 6b $(2.0 \mathrm{~g}, 20.3 \mathrm{mmol})$ and diethylamine $(4.73 \mathrm{~mL}, 45.7 \mathrm{mmol})$. Purification by flash chromatography on silica gel eluting with PE/EtOAc (7:3) afforded

1d $\left(2.7 \mathrm{~g}, 85 \%\right.$ yield) as a white solid. ${ }^{1} \mathrm{H} \mathrm{NMR}\left(\mathrm{CDCl}_{3}, 400 \mathrm{MHz}\right) \delta: 5.08$ (q, $1 \mathrm{H}, J_{\mathrm{H}, \mathrm{H}}=1.0 \mathrm{~Hz}$ ), $5.00\left(\mathrm{q}, 1 \mathrm{H}, J_{\mathrm{H}, \mathrm{H}}=1.1 \mathrm{~Hz}\right.$ ), $3.38(\mathrm{bs}, 4 \mathrm{H}), 2.32$ (tq, $2 \mathrm{H}, J=1.4 \mathrm{~Hz}, \mathrm{~J}=$ $7.4 \mathrm{~Hz}), 1.14$ (t, 6H, $J=7.1 \mathrm{~Hz}), 1.08(\mathrm{t}, 3 \mathrm{H}, J=7.4 \mathrm{~Hz}) .{ }^{13} \mathrm{C}\left\{{ }^{1} \mathrm{H}\right\} \mathrm{NMR}\left(\mathrm{CDCl}_{3}, 100.6\right.$ $\mathrm{MHz}) \delta: 172.3,147.3,111.7,42.7,27.3,14.5,12.9,11.7$. These signals are in agreement with those reported in the literature.

\section{2-cyclopentyl-N,N-diethylacrylamide (1e).}

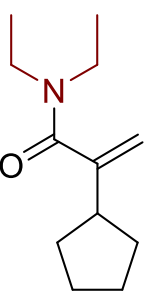

General procedure B was followed by employing 2-cyclopentylacrylic acid 6c $(1.5 \mathrm{~g}, 10.7 \mathrm{mmol})$ and diethylamine $(2.5 \mathrm{~mL}, 24.1 \mathrm{mmol})$. Purification by flash chromatography on silica gel eluting with PE/EtOAc (8:2) afforded $1 \mathrm{e}(1.7 \mathrm{~g}$, $82 \%)$ as colourless oil. ${ }^{1} \mathrm{H}$ NMR $\left(\mathrm{CDCl}_{3}, 400 \mathrm{MHz}\right) \delta: 5.08(\mathrm{q}, 1 \mathrm{H}, \mathrm{J}=0.7 \mathrm{~Hz})$, $4.98(\mathrm{t}, 1 \mathrm{H}, \mathrm{J}=1.1 \mathrm{~Hz}), 3.39(\mathrm{bs}, 4 \mathrm{H}), 2.71(\mathrm{~m}, 1 \mathrm{H}), 1.84(\mathrm{~m}, 2 \mathrm{H}), 1.42-1.74$ 
$(\mathrm{m}, 6 \mathrm{H}), 1.13(\mathrm{t}, 3 \mathrm{H}, J=6.8 \mathrm{~Hz}) .{ }^{13} \mathrm{C}\left\{{ }^{1} \mathrm{H}\right\} \mathrm{NMR}\left(\mathrm{CDCl}_{3}, 100.6 \mathrm{MHz}\right) \delta: 172.2,149.0,111.0$, 44.3, 42.7, 38.5, 31.1, 24.7, 14.3, 12.8. HRMS (ESI-TOF) m/z: $[\mathrm{M}+\mathrm{H}]+$ Calcd for $\mathrm{C}_{12} \mathrm{H}_{22} \mathrm{NO}$ 196.1701; Found 196.1698.

\section{N,N-diethyl-3-methyl-2-methylenebutanamide (1f). ${ }^{9}$}<smiles>C=C(C(=O)N(CC)CC)C(C)C</smiles>

General procedure B was followed by employing 3-methyl-2methylenebutanoic acid $6 \mathbf{a}(1.5 \mathrm{~g}, 13.2 \mathrm{mmol})$ and diethylamine $(3.1 \mathrm{~mL}, 29.6$ mmol). Purification by flash chromatography eluting with PE/EtOAc (7:3) afforded $1 \mathrm{f}(1.6 \mathrm{~g}, 73 \%)$ as colorless oil. ${ }^{1} \mathrm{H}$ NMR $\left(\mathrm{CDCl}_{3}, 400 \mathrm{MHz}\right) \delta: 5.07$ (m, 1H), $4.98(\mathrm{~m}, 1 \mathrm{H}), 3.39$ (bs, 4H), $2.66(\mathrm{~m}, 1 \mathrm{H}), 1.14$ (bs, 6H), 1.08 (d, 6H, J = 6.9 $\mathrm{Hz}) .{ }^{13} \mathrm{C}\left\{{ }^{1} \mathrm{H}\right\} \mathrm{NMR}\left(\mathrm{CDCl}_{3}, 100.6 \mathrm{MHz}\right) \delta: 172.3,151.5,110.4,42.7,38.5,31.8,20.9$, $14.3,12.8$. These signals are in agreement with those reported in the literature.

\section{2-benzyl-N,N-diethylacrylamide (1g). ${ }^{8}$}

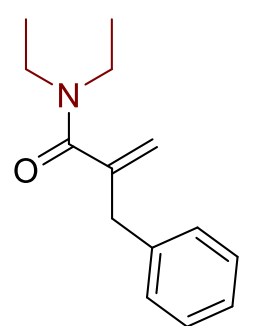

General procedure B was followed by employing 2-benzylacrylic acid $(1.6 \mathrm{~g}, 10 \mathrm{mmol})$ and diethylamine $(2.3 \mathrm{~mL}, 22.5 \mathrm{mmol})$. Purification by flash chromatography on aluminium oxide eluting with PE/EtOAc (85:15) afforded $1 \mathrm{~g}(1.6 \mathrm{~g}, 74 \%)$ as colourless oil. ${ }^{1} \mathrm{H}$ NMR $\left(\mathrm{CDCl}_{3}\right.$, $400 \mathrm{MHz}) \delta: 7.25-7.30(\mathrm{~m}, 2 \mathrm{H}), 7.18-7.22(\mathrm{~m}, 3 \mathrm{H}), 5.05(\mathrm{~m}, 1 \mathrm{H})$, $5.01(\mathrm{~m}, 1 \mathrm{H}), 3.62$ (s, 2H), 3.34 (bs, 2H), 3.09 (bs, 2H), 1.05 (bs, 3H), 0.92 (bs, 3H). ${ }^{13} \mathrm{C}\left\{{ }^{1} \mathrm{H}\right\}$ NMR $\left(\mathrm{CDCl}_{3}, 100.6 \mathrm{MHz}\right) \delta: 171.3,145.1,137.8,129.4,128.6,126.7,113.3$, $42.4,41.3,38.6,14.1,12.6$. These signals are in agreement with those reported in the literature.

\section{$\mathrm{N}, \mathrm{N}$-diethyl-2-phenylacrylamide $(\mathbf{1 h}) .^{10}$}<smiles>C=C(C(=O)N(CC)CC)c1ccccc1</smiles>

General procedure B was followed by employing 2-phenylacrylic acid (1.5 g, $10 \mathrm{mmol})$ and diethylamine $(2.3 \mathrm{~mL}, 22.5 \mathrm{mmol})$. Purification by flash chromatography on silica gel eluting with PE/EtOAc (7:3) afforded $1 \mathrm{~h}(1.4 \mathrm{~g}$, $69 \%)$ as yellow oil. ${ }^{1} \mathrm{H}$ NMR $\left(\mathrm{CDCl}_{3}, 400 \mathrm{MHz}\right) \delta$ : $1.00(\mathrm{t}, 3 \mathrm{H}, J=7.1 \mathrm{~Hz})$, $1.22(\mathrm{t}, 3 \mathrm{H}, J=7.1 \mathrm{~Hz}), 3.22(\mathrm{q}, 2 \mathrm{H}, J=7.1 \mathrm{~Hz}), 3.50$ (q, $2 \mathrm{H}, J=7.1 \mathrm{~Hz}$ ), $5.32(\mathrm{~s}, 1 \mathrm{H}), 5.69(\mathrm{~s}, 1 \mathrm{H}), 7.28-7.37(\mathrm{~m}, 3 \mathrm{H}), 7.41-7.45(\mathrm{~m}, 2 \mathrm{H}) .{ }^{13} \mathrm{C}\left\{{ }^{1} \mathrm{H}\right\} \mathrm{NMR}\left(\mathrm{CDCl}_{3}\right.$, 
$100.6 \mathrm{MHz}) \delta: 170.3,145.6,135.9,128.9,128.6,125.7,113.1,42.9,38.9,14.1,12.9$. These signals are in agreement with those reported in the literature.

\section{1-(azepan-1-yl)-2-phenylprop-2-en-1-one (1i).}

General procedure B was followed by employing 2-phenylacrylic acid (1.5<smiles>C=C(C(=O)N1CCCCCC1)c1ccccc1</smiles>
$\mathrm{g}, 10.1 \mathrm{mmol})$ and azepane $(2.6 \mathrm{~mL}, 22.7 \mathrm{mmol})$. Purification by flash chromatography eluting with PE/EtOAc (8:2) afforded $1 \mathrm{i}(1.7 \mathrm{~g}, 71 \%)$ as a colorless solid. ${ }^{1} \mathrm{H}$ NMR $\left(\mathrm{CDCl}_{3}, 400 \mathrm{MHz}\right.$ ) $\delta: 7.45(\mathrm{~m}, 2 \mathrm{H}), 7.33(\mathrm{~m}, 3 \mathrm{H})$, $5.73(\mathrm{~s}, 1 \mathrm{H}), 5.33(\mathrm{~s}, 1 \mathrm{H}), 3.64(\mathrm{~m}, 2 \mathrm{H}), 3.33(\mathrm{~m}, 2 \mathrm{H}), 1.80(\mathrm{~m}, 2 \mathrm{H}), 1.60(\mathrm{~m}$, $2 \mathrm{H}), 1.51(\mathrm{~m}, 6 \mathrm{H}) .{ }^{13} \mathrm{C}\left\{{ }^{1} \mathrm{H}\right\} \mathrm{NMR}\left(\mathrm{CDCl}_{3}, 100.6 \mathrm{MHz}\right) \delta: 170.7,145.8,135.8$, 128.8, 128.5, 125.7, 113.3, 49.1, 45.7, 29.2, 27.7, 27.4, 26.4. HRMS (ESI-TOF) m/z: [M $+\mathrm{H}]+$ Calcd for $\mathrm{C}_{15} \mathrm{H}_{20} \mathrm{NO} 230.1545$; Found 230.1539.

\section{SV. General procedure for Rh-catalyzed Asymmetric HF of a-alkyl acrylamides}

General procedure C: Rhodium catalyzed asymmetric hydroformylation of $\alpha$-alkyl acrylamides.
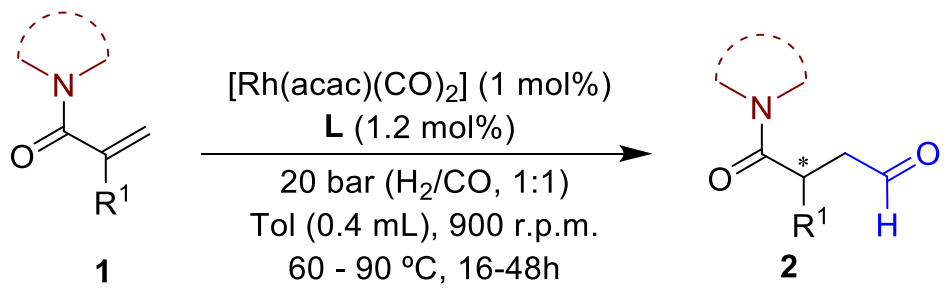

A $2 \mathrm{~mL}$ glassware reactor tube was charged with $\alpha$-alkyl acrylamide $1(0.5-0.25 \mathrm{mmol})$, $\left[\mathrm{Rh}(\mathrm{acac})(\mathrm{CO})_{2}\right](1.3 \mathrm{mg}, 0.005 \mathrm{mmol})$ and chiral ligand $\mathbf{L 6}$ or $\mathbf{L 8}(0.006 \mathrm{mmol})$ in toluene $(0.4 \mathrm{~mL})$. The reaction tube was placed in the reactor which was pressurized at the 20 bar $\left(1: 1 \mathrm{CO}: \mathrm{H}_{2}\right)$, heated to 60 or $90^{\circ} \mathrm{C}$ in an oil bath and left stirring at $900 \mathrm{rpm}$. The reaction was stopped after 48 or $16 \mathrm{~h}$ by cooling the reactor in an ice bath for $20 \mathrm{~min}$ followed by venting of the system. The mixture was purified by chromatographic column to afford the product $\mathbf{2}$. The enantiomeric excess of the resulting aldehyde $\mathbf{2}$ was analyzed by chiral HPLC or GC after reduction into the corresponding alcohol. 


\section{SVI. Optimization of the reaction conditions in the Rh-catalyzed AHF.}

Table S1: Optimization of the reaction conditions using 1a and the ligand L6.<smiles>C=C(C)C(=O)N(CC)CC</smiles>

$1 \mathrm{a}$

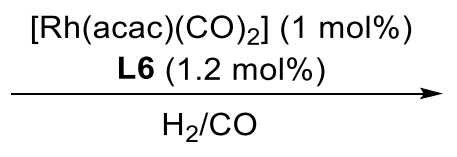<smiles>CCN(CC)C(=O)C(C)CC=[OH+]</smiles>

$2 \mathbf{a}$<smiles>CCN(CC)C(=O)C(C)(C)C=[O+]</smiles>

7<smiles>CCN1CCCC(C)C1=O</smiles>

8

\begin{tabular}{ccccccccc}
\hline Entry $^{\mathrm{a}}$ & $\mathbf{T}\left(\mathbf{}^{\mathbf{C}} \mathbf{C}\right)$ & $\mathbf{t}(\mathbf{h})$ & $\mathbf{P}\left(\mathbf{H}_{\mathbf{2}}: \mathbf{C O}\right)$ & $\mathbf{C o n v}^{\mathrm{b}}$ & $\mathbf{\% 2}^{\mathbf{a}}[\mathbf{I Y}]^{\mathrm{b}}$ & $\mathbf{7}^{\mathrm{b}}$ & $\mathbf{8}^{\mathrm{b}}$ & $\mathbf{e e}^{\mathrm{c}}$ \\
\hline $\mathbf{1}$ & 90 & 16 & $20(4: 1)$ & 99 & $36[34]$ & 0 & 63 & 80 \\
$\mathbf{2}$ & 90 & 16 & $20(1: 1)$ & 99 & $86[86]$ & 0 & 14 & 81 \\
$\mathbf{3}$ & 90 & 16 & $10(1: 1)$ & 99 & $89[86]$ & 0 & 10 & 73 \\
$\mathbf{4}$ & 60 & 16 & $20(1: 1)$ & 72 & $89[64]$ & 0 & 8 & 90 \\
$\mathbf{5}$ & 60 & 24 & $20(1: 1)$ & 81 & $89[71]$ & 0 & 9 & 89 \\
$\mathbf{6}$ & 60 & 48 & $20(1: 1)$ & 99 & $87[87]$ & 2 & 10 & 90 \\
\hline
\end{tabular}

Reaction conditions: ${ }^{\mathrm{a}} \mathbf{1 a}(0.5 \mathrm{mmol})$, toluene $(0.4 \mathrm{ml}), 900$ r.p.m. ${ }^{\text {bo }}$ Conversion and yield determined by ${ }^{1} \mathrm{H} \mathrm{NMR}$ spectroscopy using naphthalene as internal standard, values in brackets refer to isolated yields. ${ }^{\mathrm{c}} \%$ ee of $\mathbf{2 a}$ determined by GC after reduction into the alcohol.

\section{SVII. Effect of the binaphtol's configurations}

Table S2: Effect of the binaphtol's configuration using 1a and the ligand L10

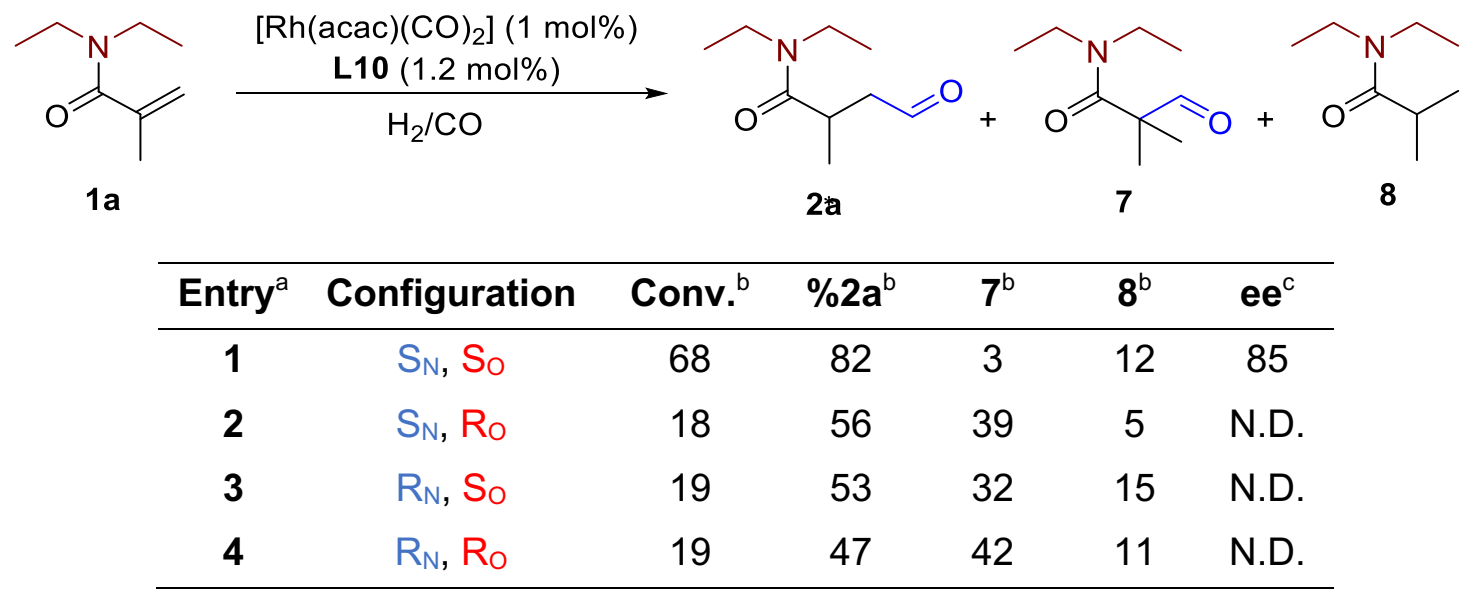

Reaction conditions: ${ }^{\mathrm{a}} \mathbf{1 a}(0.5 \mathrm{mmol}),\left[\mathrm{Rh}(\mathrm{acac})(\mathrm{CO})_{2}\right](1 \mathrm{~mol} \%), \mathrm{L} 10(1.2 \mathrm{~mol} \%), \mathrm{P}=20 \mathrm{bar}\left(\mathrm{H}_{2} / \mathrm{CO}, 1: 1\right)$, toluene $(0.4$ $\mathrm{ml}), \mathrm{T}=60^{\circ} \mathrm{C}, \mathrm{t}=16 \mathrm{~h}, 900$ r.p.m.. b\% Conversion and selectivity determined by $1 \mathrm{H}$ NMR spectroscopy using naphthalene as internal standard. $c \%$ ee of $2 a$ determined by GC after reduction into the alcohol, N.D. refers not determined. $R_{N}$ or $S_{N}$ refers to the configuration of the binaphthol at the phosphoramidite moiety and Ro or So refers to the configuration of the binaphthol at the phosphite moiety. 
SVIII. Screening of ligands for the Rh-cat. AHF of $1 f$.

Table S3: Ligand optimization for the substrate 1f.
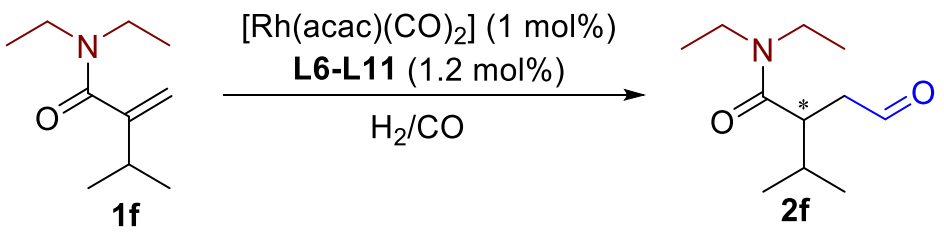

\begin{tabular}{|c|c|c|c|c|}
\hline Entry $^{a}$ & Ligand & Conversion $^{\mathrm{b}}$ & $\% 2 f[I Y]^{b}$ & $e^{c}$ \\
\hline 1 & L6 & 47 & 28 [10] & 14 \\
\hline 2 & L8 & 59 & $51[26]$ & 40 \\
\hline 3 & L10 & 78 & 77 [57] & 54 \\
\hline 4 & L9 & 60 & 53 [28] & 58 \\
\hline 5 & L7 & 36 & 33 [9] & 30 \\
\hline 6 & L11 & 44 & 36 [12] & 50 \\
\hline \multicolumn{5}{|c|}{$\begin{array}{l}\text { Reaction conditions: }{ }^{a} 1 \mathrm{f}(0.5 \mathrm{mmol}),\left[\mathrm{Rh}(\mathrm{acac})(\mathrm{CO})_{2}\right](1 \mathrm{~mol} \%), \mathrm{L}(1.2 \\
\mathrm{mol} \%), \mathrm{P}=20 \text { bar }\left(\mathrm{H}_{2} / \mathrm{CO}, 1: 1\right) \text {, toluene }(0.4 \mathrm{ml}), \mathrm{T}=90^{\circ} \mathrm{C}, \mathrm{t}=16 \mathrm{~h} \text {, } \\
900 \text { r.p.m. b\% Conversion and yield determined by }{ }^{1} \mathrm{H} \text { NMR } \\
\text { spectroscopy using naphthalene as internal standard, values in } \\
\text { brackets refer to isolated yields. }{ }^{\circ} \% \text { ee of } 2 \mathrm{f} \text { determined by GC after } \\
\text { reduction into the alcohol. }\end{array}$} \\
\hline
\end{tabular}

Table S4: Optimization of the reaction conditions using L10.
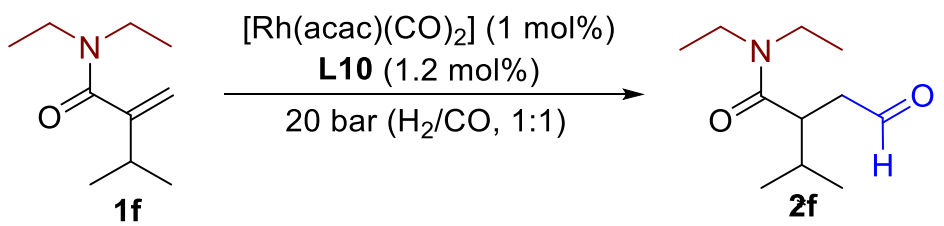

\begin{tabular}{|c|c|c|c|c|c|c|}
\hline Entry ${ }^{a}$ & Ligand & $\mathrm{T}\left({ }^{\circ} \mathrm{C}\right)$ & $t(h)$ & Conv. ${ }^{c}$ & $2 f[I Y]^{c}$ & $e^{e} e^{e}$ \\
\hline 1 & L10 & 90 & 16 & 78 & 76 [57] & 54 \\
\hline $2^{b}$ & L10 & 60 & 48 & 99 & 70 [66] & 74 \\
\hline $\begin{array}{l}\text { Reaction } \\
\text { toluene (0 } \\
\text { toluene ( } \\
\text { spectrosc }\end{array}$ & $\begin{array}{l}\text { conditions: } \\
4 \mathrm{ml} \text { ), } 900 \mathrm{r} . \mathrm{p} \\
.4 \mathrm{ml}), 900 \\
\text { ppy using nap }\end{array}$ & $\begin{array}{l}1 \mathbf{1 f}(0.5 \mathrm{~mm} \\
\text { m. }{ }^{\mathrm{b}} \mathbf{1 f}(0.25 \\
\text { r.p.m. c\% }\end{array}$ & $\begin{array}{l}\text {, }[\mathrm{Rh}(\mathrm{ac} \\
\mathrm{mol}),[\mathrm{RI} \\
\text { onversio }\end{array}$ & $\begin{array}{l}\left.(\mathrm{CO})_{2]}\right] \\
\left.\mathrm{cac}(\mathrm{CO})_{2}\right] \\
\text { and yield } \\
\text { ard, values i }\end{array}$ & $\begin{array}{l}\text { \%), L10 (1.2 } \\
\text { o\%), L10 (2. } \\
\text { rmined by }\end{array}$ & $\begin{array}{l}\text { ol \%), } \\
\text { nol \%), } \\
\text { NMR } \\
\text { olated }\end{array}$ \\
\hline
\end{tabular}

SIX. Synthesis of aldehydes via Rh-catalyzed AHF.

N,N-diethyl-2-methyl-4-oxobutanamide (2a). 


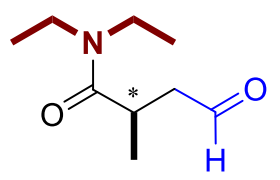

General procedure C was followed by employing N,Ndiethylmethacrylamide $(78.5 \mu \mathrm{L}, 0.5 \mathrm{mmol})$. Purification by flash chromatography on silica eluting with PE/EtOAc (1:1) afforded 2a (74.5 $\mathrm{mg}, 87 \%)$ as colourless oil. Experimental $[\alpha]_{\mathrm{D}}{ }^{25}=-4.4\left(\mathrm{c}=0.27, \mathrm{CH}_{2} \mathrm{Cl}_{2}\right) .{ }^{1} \mathrm{H} \mathrm{NMR}\left(\mathrm{CDCl}_{3}\right.$, $400 \mathrm{MHz}) \delta: 9.75(\mathrm{~s}, 1 \mathrm{H}), 3.41(\mathrm{~m}, 3 \mathrm{H}), 3.29(\mathrm{~m}, 1 \mathrm{H}), 3.10(\mathrm{~m}, 2 \mathrm{H}), 2.45(\mathrm{dd}, 1 \mathrm{H}, J=3.8$, $17.6 \mathrm{~Hz}), 1.26(\mathrm{t}, 3 \mathrm{H}, J=7.16 \mathrm{~Hz}), 1.14(\mathrm{~d}, 3 \mathrm{H}, J=6.8 \mathrm{~Hz}), 1.09(\mathrm{t}, 3 \mathrm{H}, J=7.12 \mathrm{~Hz})$. ${ }^{13} \mathrm{C}\left\{{ }^{1} \mathrm{H}\right\}$ NMR $\left(100.6 \mathrm{MHz} \mathrm{CDCl}_{3}\right) \delta: 201.4,174.5 .48 .3,42.1,40.6,30.3,18.2,14.7,13.1$. HRMS (ESI-TOF) m/z: [M + H]+ Calcd for $\mathrm{C}_{9} \mathrm{H}_{18} \mathrm{NO}_{2}$ 172.1338; Found 172.1334 .

\section{2-methyl-4-oxo-N,N-diphenylbutanamide (2b).}<smiles>CC(CC=O)C(=O)N(c1ccccc1)c1ccccc1</smiles>

General procedure C was followed by employing N,Ndiphenylmethacrylamide $1 \mathrm{~b}(118.7 \mathrm{mg}, 0.5 \mathrm{mmol})$. Purification by flash chromatography on silica eluting with PE/EtOAc (7:3) afforded 2b $(66.8 \mathrm{mg}, 50 \%)$ white solid. Experimental $[\alpha]_{D}{ }^{25}=-28.2(c=0.11$, $\mathrm{CH}_{2} \mathrm{Cl}_{2}$ ). Experimental melting point: $129-131^{\circ} \mathrm{C} .{ }^{1} \mathrm{H} \mathrm{NMR}\left(\mathrm{CDCl}_{3}, 400 \mathrm{MHz}\right) \delta: 9.76(\mathrm{~s}$, $1 \mathrm{H}), 7.17-7.52(\mathrm{~m}, 10 \mathrm{H}), 3.17(\mathrm{~m}, 1 \mathrm{H}), 3.07(\mathrm{~m}, 1 \mathrm{H}), 2.44\left(\mathrm{dd}, 1 \mathrm{H}, J=3.6 \mathrm{~Hz}, J_{\mathrm{H}, \mathrm{H}}=\right.$ $18.2 \mathrm{~Hz}), 1.14(\mathrm{~d}, 3 \mathrm{H}, J=6.8 \mathrm{~Hz}) .{ }^{13} \mathrm{C}\left\{{ }^{1} \mathrm{H}\right\} \mathrm{NMR}\left(\mathrm{CDCl}_{3}, 100.6 \mathrm{MHz}\right) \delta: 175.3,143.0$, 126.8 - 129.9, 48.5, 32.1, 200.9, 17.9. HRMS (ESI-TOF) m/z: [M + H]+ Calcd for $\mathrm{C}_{17} \mathrm{H}_{18} \mathrm{NO}_{2} 268.1338 ;$ Found 268.1340.

\section{$\mathrm{N}, \mathrm{N}$-diisopropyl-2-methyl-4-oxobutanamide (2c).}<smiles>CC(CC=O)C(=O)N(C(C)C)C(C)C</smiles>

General procedure C was followed by employing N,Ndiisopropylmethacrylamide 1c $(78.5 \mu \mathrm{L}, 0.5 \mathrm{mmol})$. Purification by flash chromatography on silica eluting with PE/EtOAc (8:2) afforded 2c $(79.7 \mathrm{mg}, 80 \%)$ as colourless oil. Experimental $[\alpha]_{D}{ }^{25}=+61.4(\mathrm{c}=$ 0.35, $\mathrm{CH}_{2} \mathrm{Cl}_{2}$ ). ${ }^{1} \mathrm{H}$ NMR $\left(\mathrm{CDCl}_{3}, 400 \mathrm{MHz}\right) \delta: 9.75(\mathrm{~s}, 1 \mathrm{H}), 4.08(\mathrm{~m}, 1 \mathrm{H}), 3.44(\mathrm{bs}, 1 \mathrm{H})$, $3.09(\mathrm{~m}, 2 \mathrm{H}), 2.39\left(\mathrm{dd}, 1 \mathrm{H}, J=4.2 \mathrm{~Hz}, J_{\mathrm{H}, \mathrm{H}}=17.5 \mathrm{~Hz}\right), 1.34(\mathrm{~m}, 6 \mathrm{H}), 1.24(\mathrm{~m}, 6 \mathrm{H}), 1.11$ $(\mathrm{d}, 3 \mathrm{H}, J=6.9 \mathrm{~Hz}) .{ }^{13} \mathrm{C}\left\{{ }^{1} \mathrm{H}\right\} \mathrm{NMR}\left(\mathrm{CDCl}_{3}, 100.6 \mathrm{MHz}\right) \delta: 201.5,174.0,48.5,48.2,45.9$, 31.8, 21.2, 21.1, 20.8, 20.6, 18.0. HRMS (ESI-TOF) m/z: [M + H]+ Calcd for $\mathrm{C}_{11} \mathrm{H}_{22} \mathrm{NO}_{2}$ 200.1651 ; Found 200.1648.

\section{N,N,2-triethyl-4-oxobutanamide (2d).}

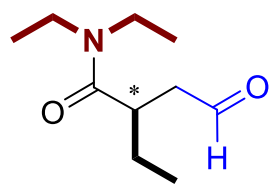

General procedure C was followed by employing N,N-diethyl-2methylenebutanamide $2 \mathbf{d}(77.5 \mathrm{mg}, 0.5 \mathrm{mmol})$. Purification by flash chromatography on silica gel eluting with PE/EtOAc (7:3) afforded 2d 
(68.5 mg, 74\%) as colourless oil. Experimental $[\alpha]_{D}{ }^{25}=-3.0\left(\mathrm{c}=0.27, \mathrm{CH}_{2} \mathrm{Cl}_{2}\right) .{ }^{1} \mathrm{H}$ NMR $\left(\mathrm{CDCl}_{3}, 400 \mathrm{MHz}\right) \delta: 9.75(\mathrm{~s}, 1 \mathrm{H}), 3.40(\mathrm{~m}, 3 \mathrm{H}), 3.26(\mathrm{~m}, 1 \mathrm{H}), 3.01(\mathrm{~m}, 2 \mathrm{H}), 2.52(\mathrm{~m}, 1 \mathrm{H})$, $1.63(\mathrm{~m}, 1 \mathrm{H}), 1.49(\mathrm{~m}, 1 \mathrm{H}), 1.24(\mathrm{t}, 3 \mathrm{H}, J=7.2 \mathrm{~Hz}), 1.08(\mathrm{t}, 3 \mathrm{H}, J=7.1 \mathrm{~Hz}), 0.90(\mathrm{t}, 3 \mathrm{H}$, $J=7.4 \mathrm{~Hz}) \cdot{ }^{13} \mathrm{C}\left\{{ }^{1} \mathrm{H}\right\} \mathrm{NMR}\left(\mathrm{CDCl}_{3}, 100.6 \mathrm{MHz}\right) \delta: 201.5,173.9,46.4,42.2,40.6,36.8$, 26.0, 14.7, 13.1, 11.7. HRMS (ESI-TOF) m/z: [M + H]+ Calcd for $\mathrm{C}_{10} \mathrm{H}_{20} \mathrm{NO}_{2}$ 186.1494; Found 186.1493.

\section{2-benzyl-N,N-diethyl-4-oxobutanamide (2e).}

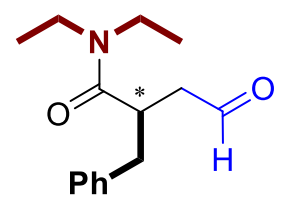

General procedure C was followed by employing 2-benzyl-N,Ndiethylacrylamide $\mathbf{1 g}(108.6 \mathrm{mg}, 0.5 \mathrm{mmol})$. Purification by flash chromatography eluting with PE/EtOAc (6:4) afforded 2 e $(80.4 \mathrm{mg}$, $65 \%)$ as yellow oil. Experimental $[\alpha]_{D}{ }^{25}=+22.3\left(\mathrm{c}=0.35, \mathrm{CH}_{2} \mathrm{Cl}_{2}\right) .{ }^{1} \mathrm{H} \mathrm{NMR}\left(\mathrm{CDCl}_{3}, 400\right.$ $\mathrm{MHz})$ : $9.72(\mathrm{~s}, 1 \mathrm{H}), 7.16-7.30(\mathrm{~m}, 5 \mathrm{H}), 3.43(\mathrm{~m}, 1 \mathrm{H}), 3.32(\mathrm{~m}, 1 \mathrm{H}), 3.15(\mathrm{~m}, 4 \mathrm{H}), 2.95$ (dd, $1 \mathrm{H}, J=7.7 \mathrm{~Hz}, J=13.3 \mathrm{~Hz}$ ), $2.68(\mathrm{dd}, 1 \mathrm{H}, J=7.4 \mathrm{~Hz}, J=13.3 \mathrm{~Hz}), 2.53(\mathrm{dd}, 1 \mathrm{H}, J$ $=3.8 \mathrm{~Hz}, J=18.4 \mathrm{~Hz}), 1.05(\mathrm{~m}, 6 \mathrm{H}) \cdot{ }^{13} \mathrm{C}\left\{{ }^{1} \mathrm{H}\right\} \mathrm{NMR}\left(\mathrm{CDCl}_{3}, 100.6 \mathrm{MHz}\right) \delta: 201.0,173.2$, 138.9, 129.3, 128.6, 126.8, 46.8, 42.0, 40.7, 39.3, 37.7, 14.2, 12.9. HRMS (ESI-TOF) $\mathrm{m} / \mathrm{z}:[\mathrm{M}+\mathrm{H}]+$ Calcd for $\mathrm{C}_{15} \mathrm{H}_{22} \mathrm{NO}_{2} 248.1651$; Found 248.1656 .

\section{N,N-diethyl-2-isopropyl-4-oxobutanamide (2f).}

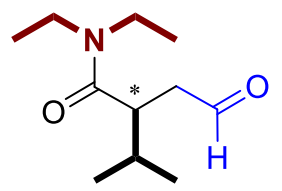

General procedure C was followed by employing N,N-diethyl-3methyl-2-methylenebutanamide $1 \mathbf{f}(42.3 \mathrm{mg}, 0.25 \mathrm{mmol})$. Purification by flash chromatography on silica gel eluting with PE/EtOAc (7:3) afforded $2 \mathbf{f}(32.8 \mathrm{mg}, 66 \%)$ as colourless oil. Experimental $[\alpha]_{\mathrm{D}}{ }^{25}=-6.0\left(\mathrm{c}=0.30, \mathrm{CH}_{2} \mathrm{Cl}_{2}\right)$. ${ }^{1} \mathrm{H} \mathrm{NMR}\left(\mathrm{CDCl}_{3}, 400 \mathrm{MHz}\right) \delta: 9.77(\mathrm{~s}, 1 \mathrm{H}), 3.48(\mathrm{~m}, 2 \mathrm{H}), 3.37(\mathrm{~m}, 1 \mathrm{H}), 3.19(\mathrm{~m}, 1 \mathrm{H}), 3.05$ $(\mathrm{m}, 1 \mathrm{H}), 2.90(\mathrm{~m}, 1 \mathrm{H}), 2.48$ (ddd, $1 \mathrm{H}, J=0.8 \mathrm{~Hz}, J=3.4 \mathrm{~Hz}, J=17.9 \mathrm{~Hz}), 1.89$ (oct, $1 \mathrm{H}$, $J=6.8 \mathrm{~Hz}), 1.25(\mathrm{t}, 3 \mathrm{H}, J=7.2 \mathrm{~Hz}), 1.09(\mathrm{t}, 3 \mathrm{H}, J=7.1 \mathrm{~Hz}), 0.92(\mathrm{~d}, 3 \mathrm{H}, J=6.8 \mathrm{~Hz})$, $0.90(\mathrm{~d}, 3 \mathrm{H}, J=6.8 \mathrm{~Hz}) .{ }^{13} \mathrm{C}\left\{{ }^{1} \mathrm{H}\right\} \mathrm{NMR}\left(\mathrm{CDCl}_{3}, 100.6 \mathrm{MHz}\right) \delta: 201.8,173.5,43.8,42.3$, 41.5, 40.4, 30.6, 21.2, 19.3, 14.5, 13.0. HRMS (ESI-TOF) m/z: [M + H]+ Calcd for $\mathrm{C}_{11} \mathrm{H}_{22} \mathrm{NO}_{2} 200.1651$; Found 200.1646.

\section{2-cyclopentyl-N,N-diethyl-4-oxobutanamide (2g).}




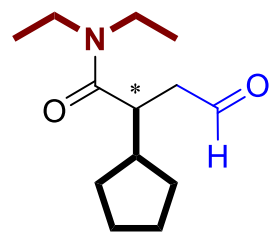

General procedure C was followed by employing 2-cyclopentyl-N,Ndiethylacrylamide 1e $(97.5 \mathrm{mg}, 0.5 \mathrm{mmol})$. Purification by flash chromatography on silica gel eluting with PE/EtOAc (7:3) afforded $\mathbf{2} \mathbf{g}$ $(79.0 \mathrm{mg}, 70 \%)$ as colourless oil. Experimental $[\alpha]_{\mathrm{D}}{ }^{25}=-26.5(\mathrm{c}=0.65$, $\left.\mathrm{CH}_{2} \mathrm{Cl}_{2}\right) .{ }^{1} \mathrm{H} \mathrm{NMR}\left(\mathrm{CDCl}_{3}, 400 \mathrm{MHz}\right) \delta: 9.75(\mathrm{~s}, 1 \mathrm{H}), 3.47(\mathrm{~m}, 3 \mathrm{H}), 3.24(\mathrm{~m}, 1 \mathrm{H}), 3.06(\mathrm{~m}$, 1H), $2.97(\mathrm{dt}, 1 \mathrm{H}, J=3.4 \mathrm{~Hz}, J=9.2 \mathrm{~Hz}), 2.54(\mathrm{ddd}, 1 \mathrm{H}, J=1.0 \mathrm{~Hz}, J=3.2 \mathrm{~Hz}, J=17.6$ $\mathrm{Hz}), 1.47-1.80(\mathrm{~m}, 6 \mathrm{H}), 2.04(\mathrm{~m}, 1 \mathrm{H}), 1.25(\mathrm{t}, 3 \mathrm{H}, J=7.2 \mathrm{~Hz}), 1.16(\mathrm{~m}, 2 \mathrm{H}), 1.09(\mathrm{t}, 3 \mathrm{H}$, $J=7.1 \mathrm{~Hz}) .{ }^{13} \mathrm{C}\left\{{ }^{1} \mathrm{H}\right\} \mathrm{NMR}\left(\mathrm{CDCl}_{3}, 100.6 \mathrm{MHz}\right) \delta: 13.0,14.5,24.9,25.1,30.3,30.7,39.8$, 40.4, 42.4, 43.5, 46.4, 173.9, 201.6. HRMS (ESI-TOF) m/z: [M + H] Calcd for $\mathrm{C}_{13} \mathrm{H}_{24} \mathrm{NO}_{2}$ 226.1807; Found 226.1809.

\section{$\mathrm{N}, \mathrm{N}$-diethyl-4-oxo-2-phenylbutanamide (2h).}<smiles>CCN(CC)C(=O)C(CC=O)c1ccccc1</smiles>

General procedure C was followed by employing N,N-diethyl-2phenylacrylamide $1 \mathrm{~h}(101.6 \mathrm{mg}, 0.5 \mathrm{mmol})$. Purification by flash chromatography eluting with PE/EtOAc (8:2) afforded 2 h $(64.3 \mathrm{mg}$, $55 \%)$ as yellow oil. Experimental $[\alpha]_{D}{ }^{25}=+25.3\left(\mathrm{c}=0.31, \mathrm{CH}_{2} \mathrm{Cl}_{2}\right) .{ }^{1} \mathrm{H}$ $\operatorname{NMR}\left(\mathrm{CDCl}_{3}, 400 \mathrm{MHz}\right) \delta: 9.79(\mathrm{~s}, 1 \mathrm{H}), 7.23-7.34(\mathrm{~m}, 5 \mathrm{H}), 4.30(\mathrm{dd}, 1 \mathrm{H}, J=4.0 \mathrm{~Hz}, J$ $=9.8 \mathrm{~Hz}), 3.49(\mathrm{~m}, 2 \mathrm{H}), 3.32(\mathrm{~m}, 1 \mathrm{H}), 3.23(\mathrm{~m}, 1 \mathrm{H}), 3.14(\mathrm{~m}, 1 \mathrm{H}), 2.62(\mathrm{dd}, 1 \mathrm{H}, J=4.0$ $\mathrm{Hz}, J=18.1 \mathrm{~Hz}), 1.07(\mathrm{t}, 3 \mathrm{H}, J=7.1 \mathrm{~Hz}), 1.02(\mathrm{t}, 3 \mathrm{H}, J=7.1 \mathrm{~Hz}) \cdot{ }^{13} \mathrm{C}\left\{{ }^{1} \mathrm{H}\right\} \mathrm{NMR}\left(\mathrm{CDCl}_{3}\right.$, 100.6 MHz) $\delta: 200.9,170.7,139.5,129.2,127.7,127.4,49.4,43.6,42.0,40.6,13.9$, 12.9. HRMS (ESI-TOF) m/z: [M + H]+ Calcd for $\mathrm{C}_{14} \mathrm{H}_{20} \mathrm{NO}_{2} 234.1494$; Found 234.1498.

\section{SX. Reduction of aldehydes to the alcohols}

General procedure D: Reduction to alcohol of the aldehydes 2 to alcohol 12.
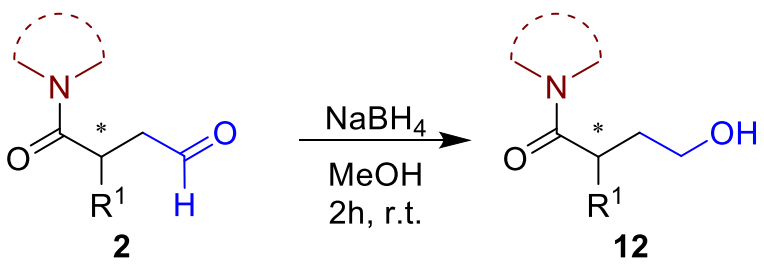

To a solution of the corresponding aldehyde $2(0.1 \mathrm{mmol})$ in methanol $(2 \mathrm{~mL})$ at $\mathrm{rt}$, sodium borohydride $(0.12 \mathrm{mmol})$ was added. After 2 hours of reaction, the reaction was quenched with water $(2 \mathrm{~mL})$ extracted with diethyl ether $(3 \times 2 \mathrm{~mL})$. The organic layers were dried over anhydrous $\mathrm{MgSO}_{4}$ and concentrated under reduced pressure. The crude was purified by flash chromatography to afford the product 12 . The enantiomeric excess of the resulting alcohols 12 was analyzed by chiral GC or HPLC.

N,N-diethyl-4-hydroxy-2-methylbutanamide (12a). 


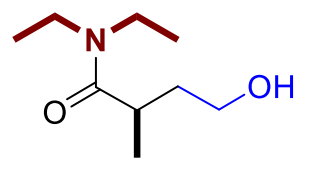

General procedure D was followed by employing N,N-diethyl-2methyl-4-oxobutanamide $2 \mathbf{a}(17 \mathrm{mg}, 0.1 \mathrm{mmol})$. Purification by flash chromatography on silica eluting with DCM afforded 12a $(16 \mathrm{mg}$, $94 \%$ ) as colourless oil. The enantiomeric excess was determined to be $90 \%$ by GC analysis on a CP-Chiralsil-Dex column $\left(100 \mathrm{kPa} \mathrm{H}, 50^{\circ} \mathrm{C}, 1^{\circ} \mathrm{C} / \mathrm{min}\right.$ until $\left.140^{\circ} \mathrm{C}\right) . t_{r \text { minor }}=$ $72.4 \mathrm{~min}, t_{r \text { major }}=74.5 \mathrm{~min} .{ }^{1} \mathrm{H}$ NMR $\left(\mathrm{CDCl}_{3}, 400 \mathrm{MHz}\right) \delta: 3.63(\mathrm{~m}, 2 \mathrm{H}), 3.35(\mathrm{~m}, 4 \mathrm{H})$, $2.89(\mathrm{~m}, 1 \mathrm{H}), 2.67(\mathrm{bs}, 1 \mathrm{H}), 1.92(\mathrm{~m}, 1 \mathrm{H}), 1.66(\mathrm{~m}, 1 \mathrm{H}), 1.19(\mathrm{t}, 3 \mathrm{H}, J=7.2 \mathrm{~Hz}), 1.14(\mathrm{~d}$, $3 \mathrm{H}, J=6.9 \mathrm{~Hz}), 1.10(\mathrm{t}, 3 \mathrm{H}, J=7.1 \mathrm{~Hz}) \cdot{ }^{13} \mathrm{C}\left\{{ }^{1} \mathrm{H}\right\} \mathrm{NMR}\left(\mathrm{CDCl}_{3}, 100.6 \mathrm{MHz}\right) \delta: 176.5,60.4$, 42.2, 40.7, 36.5, 33.0, 18.0, 15.0, 13.2. HRMS (ESI-TOF) m/z: $[M+H]+$ Calcd for $\mathrm{C}_{9} \mathrm{H}_{20} \mathrm{NO}_{2}$ 174.1494; Found 174.1485.

\section{4-hydroxy-2-methyl-N,N-diphenylbutanamide (12b).}

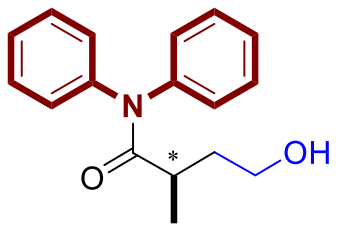

General procedure D was followed by employing 2-methyl-4-oxo$\mathrm{N}, \mathrm{N}$-diphenylbutanamide $\mathbf{2 b}(26.7 \mathrm{mg}, 0.1 \mathrm{mmol})$. Purification by flash chromatography on silica eluting with $\mathrm{DCM} / \mathrm{MeOH}(96 / 4)$ afforded $12 \mathrm{~b}$ (24.3 $\mathrm{mg}, 90 \%)$ as white solid. The enantiomeric excess was determined to be $80 \%$ by HPLC analysis on a Daicel Chiralpak IF column with a gradient 90:10 Hexane/lsopropanol, flow rate $1 \mathrm{~mL} / \mathrm{min}, \lambda=230 \mathrm{~nm}: t_{\text {rmajor }}=22.2$ $\min , t_{r \text { minor }}=25.4 \min .{ }^{1} \mathrm{H}$ NMR $\left(\mathrm{CDCl}_{3}, 400 \mathrm{MHz}\right) \delta: 7.45(\mathrm{bs}, 10 \mathrm{H}), 3.75(\mathrm{~m}, 1 \mathrm{H}), 3.63$ (m, 1H), $2.82(\mathrm{~m}, 1 \mathrm{H}), 2.02(\mathrm{~m}, 1 \mathrm{H}), 1.82(\mathrm{bs}, 1 \mathrm{H}), 1.64(\mathrm{~m}, 1 \mathrm{H}), 1.17(\mathrm{~d}, 3 \mathrm{H}, J=6.9 \mathrm{~Hz})$. ${ }^{13} \mathrm{C}\left\{{ }^{1} \mathrm{H}\right\}$ NMR $\left(\mathrm{CDCl}_{3}, 100.6 \mathrm{MHz}\right) \delta: 177.6,143.1,126.8-130.0,61.0,36.8,35.0,18.2$. HRMS (ESI-TOF) m/z: [M + H]+ Calcd for $\mathrm{C}_{17} \mathrm{H}_{20} \mathrm{NO}_{2} 270.1494$; Found 270.1488.

\section{4-hydroxy-N,N-diisopropyl-2-methylbutanamide (12c).}

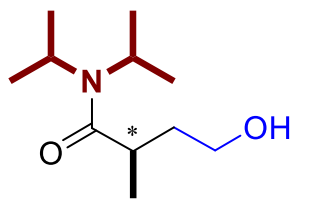

General procedure D was followed by employing N,N-diisopropyl-2methyl-4-oxobutanamide 2c (19.9 mg, $0.1 \mathrm{mmol})$. Purification by flash chromatography on silica eluting with PE/EtOAc (1:1) afforded 12c (18.5 mg, 92\%) as colourless oil. The enantiomeric excess was determined to be $99 \%$ by GC analysis on a CP-Chiralsil-Dex column $\left(100 \mathrm{kPa} \mathrm{H}_{2}, 50^{\circ} \mathrm{C}\right.$, $1^{\circ} \mathrm{C} /$ min until $\left.140^{\circ} \mathrm{C}\right) \cdot t_{r \text { minor }}=63.4 \mathrm{~min}, t_{r \text { major }}=64.9 \mathrm{~min} .{ }^{1} \mathrm{H} \mathrm{NMR}\left(\mathrm{CDCl}_{3}, 400 \mathrm{MHz}\right) \delta$ : $4.07(\mathrm{~m}, 1 \mathrm{H}), 3.53-3.69(\mathrm{~m}, 3 \mathrm{H}), 2.85(\mathrm{~m}, 1 \mathrm{H}), 2.72(\mathrm{bs}, 1 \mathrm{H}), 1.95(\mathrm{~m}, 1 \mathrm{H}), 1.65(\mathrm{~m}$, $1 \mathrm{H}), 1.35(\mathrm{~m}, 6 \mathrm{H}), 1.23(\mathrm{t}, 6 \mathrm{H}, J=6.5 \mathrm{~Hz}), 1.12(\mathrm{~d}, 3 \mathrm{H}, J=7.0 \mathrm{~Hz}) \cdot{ }^{13} \mathrm{C}\left\{{ }^{1} \mathrm{H}\right\} \mathrm{NMR}\left(\mathrm{CDCl}_{3}\right.$, $100.6 \mathrm{MHz}$ ) $\delta: 176.3,60.7,48.2,46.0,36.4,34.8,21.4,20.8,20.7,17.9$. HRMS (ESITOF) m/z: [M+ H]+ Calcd for $\mathrm{C}_{11} \mathrm{H}_{24} \mathrm{NO}_{2} 202.1807$; Found 202.1810.

N,N,2-triethyl-4-hydroxybutanamide (12d). 


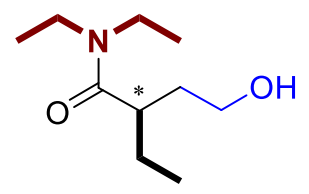

General procedure D was followed by employing N,N,2-triethyl-4oxobutanamide 2d (18.5 mg, $0.1 \mathrm{mmol})$. Purification by flash chromatography on silica eluting with $\mathrm{DCM} / \mathrm{MeOH}$ (98:2) afforded $12 \mathrm{~d}(17.6 \mathrm{mg}, 94 \%)$ as colourless oil. The enantiomeric excess was determined to be $90 \%$ by $\mathrm{GC}$ analysis on a CP-Chiralsil-Dex column $\left(100 \mathrm{kPa} \mathrm{H}_{2}, 50^{\circ} \mathrm{C}, 1^{\circ} \mathrm{C} / \mathrm{min}\right.$ until $\left.140^{\circ} \mathrm{C}\right) . t_{r \text { minor }}=69.6 \mathrm{~min}, t_{r \text { major }}=71.1 \mathrm{~min} .{ }^{1} \mathrm{H} \mathrm{NMR}\left(\mathrm{CDCl}_{3}, 400 \mathrm{MHz}\right) \delta: 3.64(\mathrm{~m}, 2 \mathrm{H})$, $3.39(\mathrm{~m}, 4 \mathrm{H}), 2.73(\mathrm{~m}, 1 \mathrm{H}), 2.39(\mathrm{bs}, 1 \mathrm{H}), 1.90(\mathrm{~m}, 1 \mathrm{H}), 1.74(\mathrm{~m}, 2 \mathrm{H}), 1.52(\mathrm{~m}, 1 \mathrm{H}), 1.21$ $(\mathrm{t}, 3 \mathrm{H}, J=7.2 \mathrm{~Hz}), 1.13(\mathrm{t}, 3 \mathrm{H}, J=7.1 \mathrm{~Hz}), 0.91(\mathrm{t}, 3 \mathrm{H}, J=7.4 \mathrm{~Hz}) \cdot{ }^{13} \mathrm{C}\left\{{ }^{1} \mathrm{H}\right\} \mathrm{NMR}\left(\mathrm{CDCl}_{3}\right.$, $100.6 \mathrm{MHz}) \delta: 175.9,60.6,42.3,40.8,40.0,34.8,25.9,15.0,13.2,12.2$. HRMS (ESITOF) $\mathrm{m} / \mathrm{z}:[\mathrm{M}+\mathrm{H}]+$ Calcd for $\mathrm{C}_{10} \mathrm{H}_{22} \mathrm{NO}_{2}$ 188.1651; Found 188.1649.

\section{2-benzyl-N,N-diethyl-4-hydroxybutanamide (12e).}

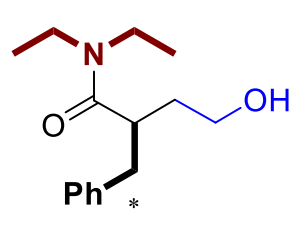

General procedure D was followed by employing 2-benzyl-N,Ndiethyl-4-oxobutanamide $2 \mathrm{e}(23.1 \mathrm{mg}, 0.1 \mathrm{mmol})$. Purification by flash chromatography on silica eluting with $\mathrm{DCM} / \mathrm{MeOH}(97 / 3)$ afforded 12e (23.6 $\mathrm{mg}, 95 \%)$ as colourless oil. The enantiomeric excess was determined to be $86 \%$ by HPLC analysis on a Daicel Chiralpak OD-H column with a gradient 80:20 Hexane/Isopropanol, flow rate $1 \mathrm{~mL} / \mathrm{min}, \lambda=230 \mathrm{~nm}: t_{\text {r major }}=4.3$ $\min , t_{r \text { minor }}=5.2 \min .{ }^{1} \mathrm{H} \mathrm{NMR}\left(\mathrm{CDCl}_{3}, 400 \mathrm{MHz}\right) \delta: 7.16-7.26(\mathrm{~m}, 5 \mathrm{H}), 3.66(\mathrm{bs}, 2 \mathrm{H})$, $3.38(\mathrm{~m}, 1 \mathrm{H}), 3.22(\mathrm{~m}, 1 \mathrm{H}), 3.04(\mathrm{~m}, 4 \mathrm{H}), 2.71(\mathrm{~m}, 1 \mathrm{H}), 2.42(\mathrm{bs}, 1 \mathrm{H}), 1.99(\mathrm{~m}, 1 \mathrm{H}), 1.77$ $(\mathrm{m}, 1 \mathrm{H}), 1.02$ (t, 3H, J = 7.2 Hz), 0.89 (t, 3H, J = 7.2 Hz). ${ }^{13} \mathrm{C}\left\{{ }^{1} \mathrm{H}\right\}$ NMR $\left(\mathrm{CDCl}_{3}, 100.6\right.$ $\mathrm{MHz}) \delta: 173.9,138.9,128.3,127.5,125.4,59.4,41.0,40.0,39.9,38.5,34.6,13.4,12.0$. HRMS (ESI-TOF) m/z: [M + H]+ Calcd for $\mathrm{C}_{15} \mathrm{H}_{24} \mathrm{NO}_{2} 250.1807$; Found 250.1807.

\section{N,N-diethyl-4-hydroxy-2-isopropylbutanamide (12f).}

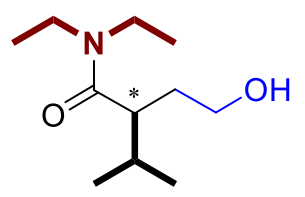

General procedure D was followed by employing N,N-diethyl-2isopropyl-4-oxobutanamide $2 \mathrm{f}(19.9 \mathrm{mg}, 0.1 \mathrm{mmol})$. Purification by flash chromatography on silica eluting with $\mathrm{DCM} / \mathrm{MeOH}(96 / 4)$ afforded $12 \mathrm{f}$ (18.3 $\mathrm{mg}, 91 \%$ ) as colourless oil. The enantiomeric excess was determined to be $74 \%$ by $\mathrm{GC}$ analysis on a CP-Chiralsil-Dex column $\left(100 \mathrm{kPa} \mathrm{H}_{2}, 50^{\circ} \mathrm{C}, 1^{\circ} \mathrm{C} / \mathrm{min}\right.$ until $\left.140^{\circ} \mathrm{C}\right) . t_{r \text { minor }}=71.5 \mathrm{~min}, t_{r \text { major }}=73.4 \mathrm{~min} .{ }^{1} \mathrm{H} \mathrm{NMR}\left(\mathrm{CDCl}_{3}, 400 \mathrm{MHz}\right) \delta: 3.64(\mathrm{~m}$, 2H), 3.39 (m, 4H), 2.55 (bs, 1H), 2.49 (td, $1 \mathrm{H}, J=3.0 \mathrm{~Hz}, J=8.9 \mathrm{~Hz}), 1.96(\mathrm{~m}, 2 \mathrm{H}), 1.75$ $(\mathrm{m}, 1 \mathrm{H}), 1.20(\mathrm{t}, 3 \mathrm{H}, J=7.1 \mathrm{~Hz}), 1.12(\mathrm{t}, 3 \mathrm{H}, J=7.1 \mathrm{~Hz}), 0.95(\mathrm{~d}, 3 \mathrm{H}, J=6.7 \mathrm{~Hz}), 0.91$ $(\mathrm{d}, 3 \mathrm{H}, J=6.7 \mathrm{~Hz}) .{ }^{13} \mathrm{C}\left\{{ }^{1} \mathrm{H}\right\} \mathrm{NMR}\left(\mathrm{CDCl}_{3}, 100.6 \mathrm{MHz}\right) \delta: 175.8,60.7,45.3,42.4,40.8$, 32.3, 30.3, 21.5, 20.0, 14.9, 13.1. HRMS (ESI-TOF) m/z: [M + H]+ Calcd for $\mathrm{C}_{11} \mathrm{H}_{24} \mathrm{NO}_{2}$ 202.1807; Found 202.1803. 
2-cyclopentyl-N,N-diethyl-4-hydroxybutanamide (12g).

General procedure D was followed by employing 2-cyclopentyl-N,N-diethyl-4-

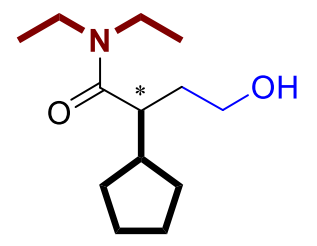
oxobutanamide $2 \mathrm{~g}(22.5 \mathrm{mg}, 0.1 \mathrm{mmol})$. Purification by flash chromatography on silica eluting with $\mathrm{DCM} / \mathrm{MeOH}(98 / 2)$ afforded $12 \mathrm{~g}(20.2 \mathrm{mg}, 89 \%)$ as colourless oil. The enantiomeric excess was determined to be $82 \%$ by GC analysis on a CP-Chiralsil-Dex column $\left(100 \mathrm{kPa} \mathrm{H}, 50^{\circ} \mathrm{C}, 1^{\circ} \mathrm{C} / \mathrm{min}\right.$ until $\left.160^{\circ} \mathrm{C}\right) . t_{r \text { minor }}=96.0 \mathrm{~min}, t_{r \text { major }}=97.3 \mathrm{~min} .{ }^{1} \mathrm{H} \mathrm{NMR}$ $\left(\mathrm{CDCl}_{3}, 400 \mathrm{MHz}\right) \delta: 3.64(\mathrm{~m}, 2 \mathrm{H}), 3.44(\mathrm{~m}, 3 \mathrm{H}), 3.32(\mathrm{~m}, 1 \mathrm{H}), 2.67$ (bs, $\left.1 \mathrm{H}\right), 2.61$ (dt, $1 \mathrm{H}, J=3.3 \mathrm{~Hz}, J=9.3 \mathrm{~Hz}), 2.20(\mathrm{~m}, 1 \mathrm{H}), 1.49-1.95(\mathrm{~m}, 8 \mathrm{H}), 1.19(\mathrm{t}, 3 \mathrm{H}, J=7.2 \mathrm{~Hz})$, $1.04-1.16(\mathrm{~m}, 5 \mathrm{H}) .{ }^{13} \mathrm{C}\left\{{ }^{1} \mathrm{H}\right\}$ NMR $\left(\mathrm{CDCl}_{3}, 100.6 \mathrm{MHz}\right) \delta: 176.1,60.5,43.8,42.4,40.8$, $34.3,31.3,30.6,25.1,25.0,15.0,13.1$. HRMS (ESI-TOF) m/z: $[M+H]+$ Calcd for $\mathrm{C}_{13} \mathrm{H}_{26} \mathrm{NO}_{2} 228.1964 ;$ Found 228.1966.

\section{N,N-diethyl-4-hydroxy-2-phenylbutanamide (12h).}

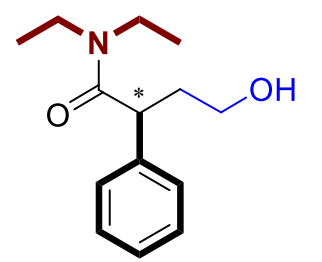

General procedure D was followed by N,N-diethyl-4-oxo-2phenylbutanamide $2 \mathrm{~h}(23.3 \mathrm{mg}, 0.1 \mathrm{mmol})$. Purification by flash chromatography on silica eluting with PE/EtOAc (1:1) afforded 12h (21.9 $\mathrm{mg}, 93 \%)$ as colourless oil. The enantiomeric excess was determined to be $78 \%$ by HPLC analysis on a Daicel Chiralpak ID column with a gradient $80: 20$ Hexane/lsopropanol, flow rate $1 \mathrm{~mL} / \mathrm{min}, \lambda=230 \mathrm{~nm}: t_{r \text { major }}=7.2 \mathrm{~min}, t_{r \text { major }}=8.9$ min. ${ }^{1} \mathrm{H}$ NMR $\left(\mathrm{CDCl}_{3}, 400 \mathrm{MHz}\right) \delta: 7.21-7.34(\mathrm{~m}, 5 \mathrm{H}), 3.97(\mathrm{~m}, 1 \mathrm{H}), 3.59(\mathrm{~m}, 2 \mathrm{H}), 3.48$ (m, 1H), $3.28(\mathrm{~m}, 2 \mathrm{H}), 3.14(\mathrm{~m}, 1 \mathrm{H}), 2.69(\mathrm{bs}, 1 \mathrm{H}), 2.32(\mathrm{~m}, 1 \mathrm{H}), 1.97(\mathrm{~m}, 1 \mathrm{H}), 1.09(\mathrm{t}$, $3 \mathrm{H}, J=7.2 \mathrm{~Hz}), 0.98(\mathrm{t}, 3 \mathrm{H}, J=7.2 \mathrm{~Hz}) .{ }^{13} \mathrm{C}\left\{{ }^{1} \mathrm{H}\right\} \mathrm{NMR}\left(\mathrm{CDCl}_{3}, 100.6 \mathrm{MHz}\right) \delta: 172.8$. 140.2, 129.0, 127.9, 127.1, 60.8, 46.8, 42.1, 40.7, 37.8, 14.2, 12.9. HRMS (ESI-TOF) $\mathrm{m} / \mathrm{z}:[\mathrm{M}+\mathrm{H}]+$ Calcd for $\mathrm{C}_{14} \mathrm{H}_{22} \mathrm{NO}_{2} 236.1651$; Found 236.1649.

\section{1-(azepan-1-yl)-4-hydroxy-2-phenylbutan-1-one (12i).}

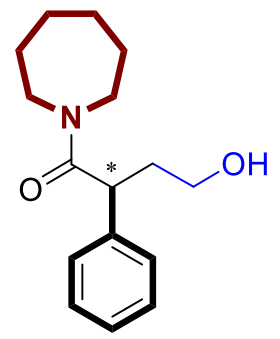

General procedure C was followed by employing 1-(azepan-1-yl)-2phenylprop-2-en-1-one 1i (114.6 mg, $0.5 \mathrm{mmol}$ ). The amount of aldehyde $2 \mathbf{i}$ was quantified by ${ }^{1} \mathrm{H}$ NMR in the reaction crude using naphthalene as an internal standard. The reaction crude was filtered to a path of silica and eluting with DCM, the corresponding solution was concentrated under reduced pressure. The crude was diluted with $\mathrm{MeOH}$ (2 mL MeOH/0.1 mmol aldehyde $2 \mathrm{i}$ ) and the sodium borohydride (1.2 eq.) was added. After 2 hours of reaction at room temperature, the reaction was quenched with water and extracted with diethyl ether. The organic layers were dried over anhydrous 
$\mathrm{MgSO}_{4}$ and concentrated under reduced pressure. The crude was purified by flash chromatography on silica eluting with $\mathrm{DCM} / \mathrm{MeOH}(96 / 4)$ to afford the product $12 \mathbf{i}(68.0$ $\mathrm{mg}, 52 \%)$ as colourless oil. The enantiomeric excess was determined to be $74 \%$ by HPLC analysis on a Daicel Chiralpak IF column with a gradient 92:08 Hexane/Isopropanol, flow rate $1 \mathrm{~mL} / \mathrm{min}, \lambda=230 \mathrm{~nm}$ : $t_{r \text { minor }}=22.9 \mathrm{~min}, t_{\text {rmajor }}=24.4 \mathrm{~min}$. Experimental $[\alpha]_{D}{ }^{25}=+30.9\left(\mathrm{c}=0.35, \mathrm{CH}_{2} \mathrm{Cl}_{2}\right) .{ }^{1} \mathrm{H} \mathrm{NMR}\left(\mathrm{CDCl}_{3}, 400 \mathrm{MHz}\right) \delta: 7.21-7.33$ $(\mathrm{m}, 5 \mathrm{H}), 4.00(\mathrm{~m}, 1 \mathrm{H}), 3.60(\mathrm{~m}, 3 \mathrm{H}), 3.46(\mathrm{~m}, 2 \mathrm{H}), 3.27(\mathrm{~m}, 1 \mathrm{H}), 2.70(\mathrm{bs}, 1 \mathrm{H}), 2.34(\mathrm{~m}$, $1 \mathrm{H}), 1.97(\mathrm{~m}, 1 \mathrm{H}), 1.21-1.78(\mathrm{~m}, 8 \mathrm{H}) .{ }^{13} \mathrm{C}\left\{{ }^{1} \mathrm{H}\right\} \mathrm{NMR}\left(\mathrm{CDCl}_{3}, 100.6 \mathrm{MHz}\right) \delta: 127.1,173.3$, 140.2, 129.0, 128.0, 60.9, 48.1, 47.0, 46.6, 37.8, 29.1, 27.4, 27.2, 26.4. HRMS (ESITOF) $\mathrm{m} / \mathrm{z}:[\mathrm{M}+\mathrm{H}]+$ Calcd for $\mathrm{C}_{16} \mathrm{H}_{24} \mathrm{NO}_{2} 262.1807$; Found 262.1804.

\section{SXI. General procedure for Rh-catalyzed asymmetric hydroaminomethylation of $\alpha$-alkyl acrylamides}

General procedure E: rhodium catalyzed asymmetric HAM of a-alkyl acrylamides.<smiles>[R]C(=C)C(=O)N1CCCCC1</smiles>

1

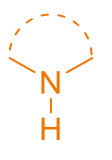

3

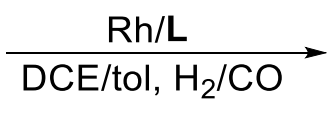

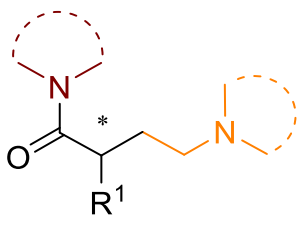

4

A $2 \mathrm{~mL}$ glassware reactor tube was charged with $\alpha$-alkyl acrylamide $1(0.5-0.25 \mathrm{mmol})$, amine $3(0.5-0.25 \mathrm{mmol})$, [Rh(acac)(CO) $]$ (1.3 mg, $0.005 \mathrm{mmol})$ in DCE $(0.2 \mathrm{~mL})$ and chiral ligand $\mathbf{L}(0.006 \mathrm{mmol})$ in toluene $(0.2 \mathrm{~mL})$. The reaction tube was placed in the reactor, which was pressurized at the desired pressure, heated to $90^{\circ} \mathrm{C}$ in an oil bath and left stirring at $900 \mathrm{rpm}$. The reaction was stopped after desired time by cooling the reactor in an ice bath for 20 min followed by venting of the system. The mixture was purified by chromatographic column and the enantiomeric excess of the resulting $\alpha$-alkylY-aminobutyric amides 4 analyzed by chiral HPLC. 


\section{SXII. Optimization of the reaction conditions in the Rh-catalyzed HAM}

Table S5. Optimization of the reaction conditions using substrate 1a, morpholine 3a and ligand L6.

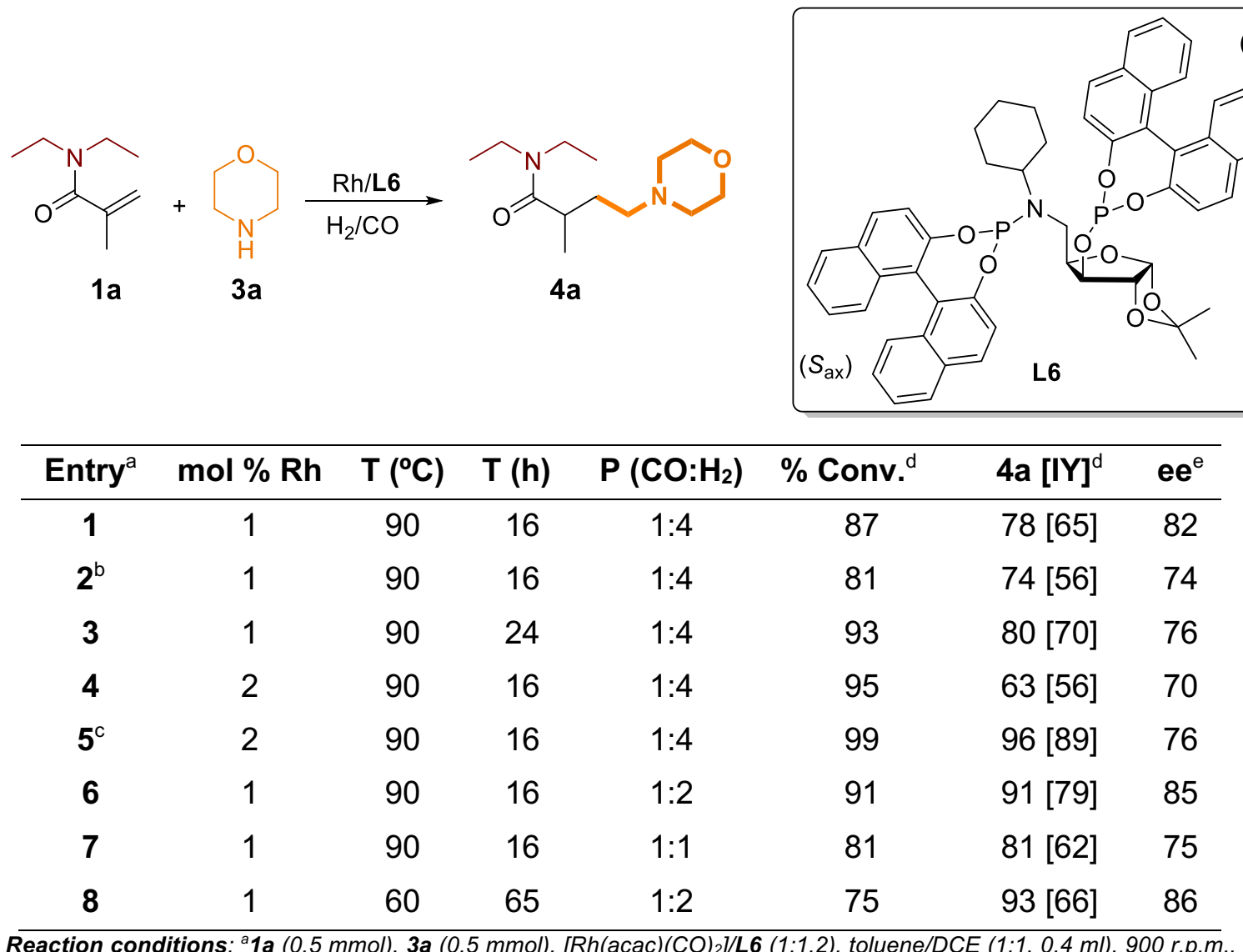

${ }^{b}\left[R h(C O D)_{2}\right] B F_{4}{ }^{c} 1 a(0.25 \mathrm{mmol}), 3 a(0.25 \mathrm{mmol}) .{ }^{\mathrm{c}} \%$ Conversion and selectivity determined by ${ }^{1} \mathrm{H}$ NMR spectroscopy

using naphthalene as internal standard, values in brackets refer to isolated yields. ${ }^{e} \%$ ee of 4 a determined by HPLC.

\section{SXIII. Optimization of reaction conditions for substrate $1 \mathrm{~h}$}

Table S6. Optimization of the reaction conditions using substrate 1h, morpholine 3a and ligand L6.

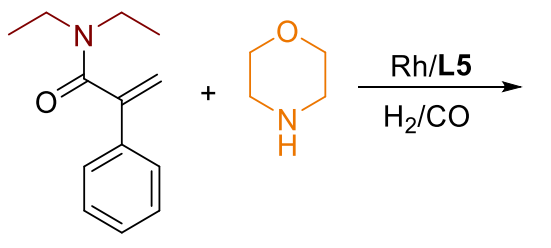

$1 \mathrm{~h}$

$3 a$

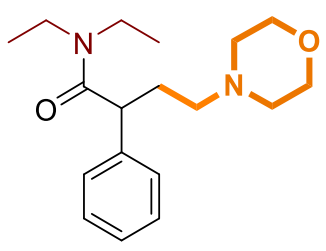

4j

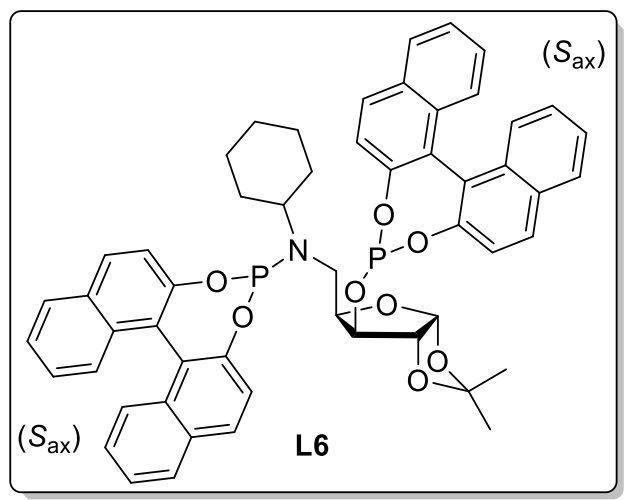

\begin{tabular}{|c|c|c|c|c|c|c|}
\hline Entry $^{a}$ & $\mathrm{~T}\left({ }^{\circ} \mathrm{C}\right)$ & $T(h)$ & $\mathrm{P}\left(\mathrm{CO}: \mathrm{H}_{2}\right)$ & $\%$ Conv. $^{c}$ & $4 \mathrm{j}[\mathrm{IY}]^{\mathrm{c}}$ & $\mathbf{e e}^{\mathrm{d}}$ \\
\hline 1 & 90 & 16 & $1: 4$ & 34 & 77 [12] & 78 \\
\hline 2 & 90 & 65 & $1: 4$ & 43 & 86 [24] & 76 \\
\hline 3 & 90 & 16 & $1: 2$ & 43 & 86 [30] & 73 \\
\hline 4 & 90 & 16 & 1:1 & 28 & 72 [20] & 70 \\
\hline
\end{tabular}




$\begin{array}{lllllll}\mathbf{5}^{\mathrm{b}} & 90 & 16 & 1: 4 & 81 & 82[66] & 76 \\ \mathbf{6}^{\mathrm{b}} & 90 & 16 & 1: 2 & 51 & 84[38] & 74 \\ \mathbf{7}^{\mathrm{b}} & 60 & 48 & 1: 4 & 22 & 82[18] & 80\end{array}$

Reaction conditions: ${ }^{a} \mathbf{h h}(0.5 \mathrm{mmol}), 3 a(0.5 \mathrm{mmol})$, [Rh(acac)(CO) 2 ] (1 mol\%), L6 (1.2 mol \%), toluene (0.4 ml), 900 r.p.m. ${ }^{b} \mathbf{1 h}(0.25 \mathrm{mmol}), 3 a(0.25 \mathrm{mmol}),\left[R h(\mathrm{acac})(\mathrm{CO})_{2}\right]$ (2 mol\%), L6 (2.4 mol \%), toluene (0.4 ml), 900 r.p.m. ${ }^{c \%}$ Conversion and selectivity determined by ${ }^{1} \mathrm{H}$ NMR spectroscopy using naphthalene as internal standard, values in brackets refer to isolated yields. ${ }^{d \%}$ ee of $4 j$ determined by HPLC.

\section{SXIV. Optimization of reaction conditions for substrate $1 \mathrm{i}$}

Table S7: Optimization of the reaction conditions using substrate 1i, morpholine 3 a and ligand L6.

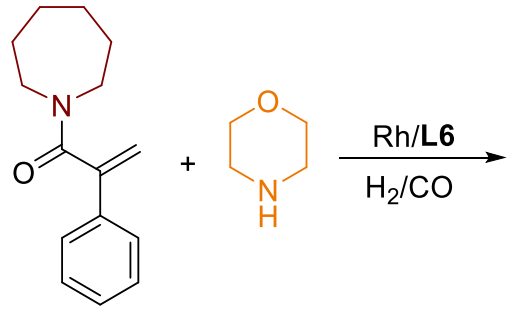

1i

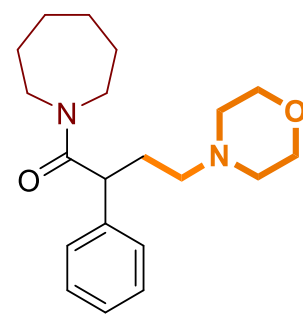

4k

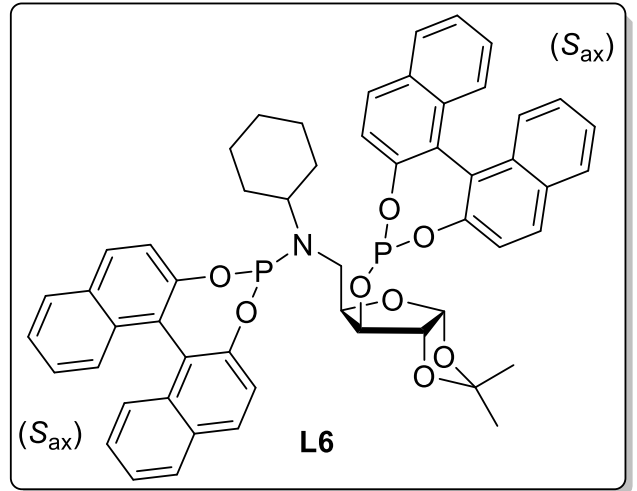

\begin{tabular}{ccccccc}
\hline Entry $^{\mathrm{a}}$ & $\mathbf{T}\left(\mathbf{~}^{\mathbf{}} \mathbf{C}\right)$ & $\mathbf{T}(\mathbf{h})$ & $\mathbf{P}\left(\mathbf{C O}: \mathbf{H}_{\mathbf{2}}\right)$ & $\mathbf{~ \% ~ C o n v . ~}^{\mathrm{c}}$ & $\mathbf{4 k ~ [ I Y ~}^{\mathrm{c}}$ & $\mathbf{~ e e}^{\mathrm{d}}$ \\
\hline $\mathbf{1}$ & 90 & 16 & $1: 4$ & 30 & $72[19]$ & 74 \\
$\mathbf{2}$ & 90 & 16 & $1: 2$ & 54 & $86[39]$ & 76 \\
$\mathbf{3}$ & 90 & 16 & $1: 1$ & 36 & $61[20]$ & 72 \\
$\mathbf{4}^{\mathrm{b}}$ & 90 & 16 & $1: 2$ & 71 & $90[59]$ & 84 \\
$\mathbf{5}^{\mathrm{b}}$ & 60 & 48 & $1: 2$ & 8 & N.D. & N.D.
\end{tabular}

Reaction conditions: ${ }^{a} 1 \mathbf{1}(0.5 \mathrm{mmol}), 3 a(0.5 \mathrm{mmol}),\left[R h(a c a c)(\mathrm{CO})_{2}\right](1 \mathrm{~mol} \%)$, L6 (1.2 mol \%), toluene $(0.4 \mathrm{ml}), 900$ r.p.m. ${ }^{b}$ 1i (0.25 mmol), 3a (0.25 mmol), [Rh(acac)(CO) 2 (2 mol\%), L6 (2.4 mol \%), toluene (0.4 ml), 900 r.p.m. ${ }^{c \%}$ Conversion and selectivity determined by ${ }^{1} \mathrm{H}$ NMR spectroscopy using naphthalene as internal standard, values in brackets refer to isolated yields. ${ }^{d} \%$ ee of $\mathbf{4} \boldsymbol{k}$ determined by HPLC.

\section{SXV. Optimization of the reaction conditions for the synthesis of RWAY (4I)}

Table S8: Optimization of the reaction conditions using substrate $\mathbf{1 i}$, amine $\mathbf{3 b}$ and the ligand $\mathbf{L 6}$.<smiles>C=C(C(=O)N1CCCCCC1)c1ccccc1</smiles>

$1 \mathbf{i}$

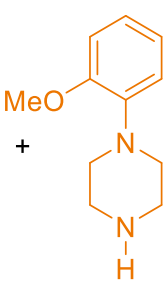

$3 b$

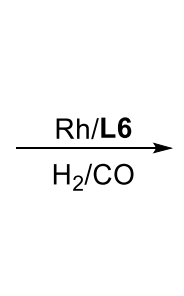

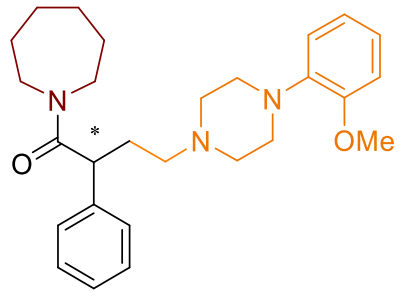

RWAY 4I 


\begin{tabular}{|c|c|c|c|c|c|c|c|}
\hline Entry $^{a}$ & Solvent (1:1) & $\% \mathrm{Rh}$ & $\mathrm{T}\left({ }^{\circ} \mathrm{C}\right)$ & $T(h)$ & $\%$ Conv. $^{c}$ & $\% 4 \mathrm{IIYY}]^{\mathrm{C}}$ & $\%$ ee $^{d}$ \\
\hline 1 & DCE/Tol & 1 & 90 & 16 & 15 & - & - \\
\hline $3^{b}$ & DCE/Tol & 2 & 90 & 16 & 30 & - & - \\
\hline $3^{b}$ & t-Butanol/Tol & 2 & 90 & 16 & 51 & 75 [30] & 72 \\
\hline $4^{b}$ & t-Butanol/Tol & 2 & 80 & 65 & 66 & 79 [48] & 76 \\
\hline $5^{b}$ & 2-MeTHF/Tol & 2 & 80 & 65 & 84 & 80 [64] & 82 \\
\hline
\end{tabular}

Reaction conditions: ${ }^{a} \mathbf{1 i}(0.5 \mathrm{mmol})$, amine $\mathbf{3 b}(0.5 \mathrm{mmol}),\left[\mathrm{Rh}(\mathrm{acac})(\mathrm{CO})_{2}\right](1 \mathrm{~mol} \%), \mathbf{L 6}$ (1.2 mol \%), $\mathrm{P}=20 \mathrm{bar}\left(\mathrm{H}_{2} / \mathrm{CO}\right.$, 2:1), solvent $0.4 \mathrm{ml}, 900$ r.p.m.. ${ }^{b} \mathbf{1 i}(0.25 \mathrm{mmol})$, amine $3 \mathrm{~b}(0.25 \mathrm{mmol}),\left[R h(\mathrm{acac})(\mathrm{CO}){ }_{2}\right](2 \mathrm{~mol} \%), \mathbf{L} 6(2.4 \mathrm{~mol} \%), P=$ 20 bar $\left(H_{2} / C O, 2: 1\right)$, solvent 0.4 ml, 900 r.p.m.. ${ }^{c \%}$ Conversion and selectivity determined by ${ }^{1} \mathrm{H}$ NMR spectroscopy using naphthalene as internal standard, values in brackets refer to isolated yields. ${ }^{d} \%$ ee of $4 \mathbf{I}$ determined by HPLC.

\section{SXVI. Experiment at $1 \mathrm{mmol}$ scale}

Table S9: Rhodium catalyzed asymmetric HAM of $\mathbf{1 a}$ at $1 \mathrm{mmol}$ scale using amine $3 \mathbf{a}$ and ligand L6.

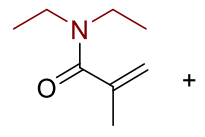

$1 \mathbf{a}$

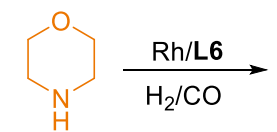

$3 \mathbf{a}$

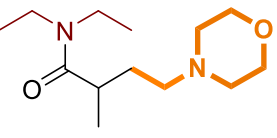

$4 \mathbf{a}$

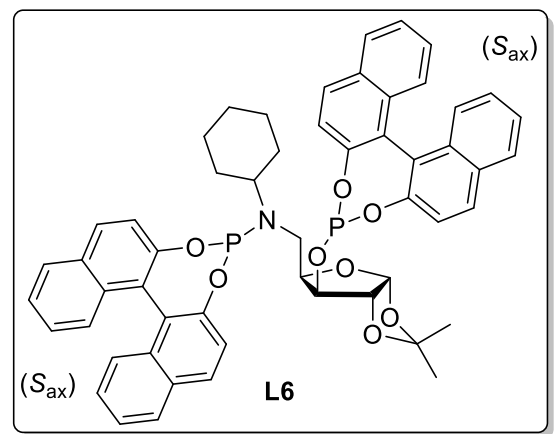

A $2 \mathrm{~mL}$ glassware reactor tube was charged with $1 \mathrm{a}(157 \mu \mathrm{L}, 1.0 \mathrm{mmol})$, amine $3 \mathrm{a}(87.4$ $\mu \mathrm{L}, 1.0 \mathrm{mmol}),\left[\mathrm{Rh}(\mathrm{acac})(\mathrm{CO})_{2}\right](2.6 \mathrm{mg}, 0.01 \mathrm{mmol})$ in $\mathrm{DCE}(0.4 \mathrm{~mL})$ and chiral ligand L6 $(10.8 \mathrm{mg}, 0.012 \mathrm{mmol})$ in toluene $(0.4 \mathrm{~mL})$. The reaction tube was placed in the reactor, which was pressurized at 20 bar $\left(1: 2 \mathrm{CO} / \mathrm{H}_{2}\right)$, heated to $90^{\circ} \mathrm{C}$ in an oil bath and left stirring at $900 \mathrm{rpm}$. The reaction was stopped after $16 \mathrm{~h}$ by cooling the reactor in an ice bath for $20 \mathrm{~min}$ followed by venting of the system. The reaction mixture was analyzed by ${ }^{1} \mathrm{H}$ NMR using naphthalene as internal standard. The mixture was purified by chromatographic column and the enantiomeric excess of the resulting $4 \mathbf{a}$ analyzed by chiral HPLC.

\begin{tabular}{cccc}
\hline Entry & \% $^{\text {Conv. }}{ }^{\mathrm{a}}$ & ${\text { 4a }[\mathrm{IY}]^{\mathrm{a}}}$ & \% ee $^{\mathrm{b}}$ \\
\hline $\mathbf{1}$ & 97 & $79[73]$ & 82
\end{tabular}

a\% Conversion and selectivity determined by ${ }^{1} \mathrm{H}$ NMR spectroscopy using naphthalene as internal standard, values in brackets refer to isolated yields. ${ }^{b} \%$ ee of $\mathbf{4 a}$ determined by HPLC.

SXVII.Synthesis of $\alpha$-alkyl-y-aminobutyric amides via Rh-catalyzed asymmetric HAM.

N,N-diethyl-2-methyl-4-morpholinobutanamide (4a). 


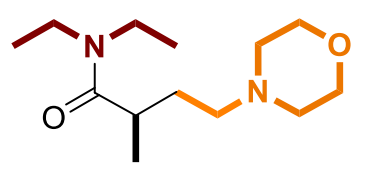

General procedure E was followed by employing N,Ndiethylmethacrylamide $1 \mathrm{a}(78.5 \mu \mathrm{L}, 0.5 \mathrm{mmol})$ and morpholine (43.7 $\mu \mathrm{L}, 0.5 \mathrm{mmol})$. Purification by flash chromatography on silica eluting with $\mathrm{DCM} / \mathrm{MeOH}(95: 5)$ afforded $\mathbf{4 a}(95.8 \mathrm{mg}, 79 \%)$ as yellow oil. The enantiomeric excess was determined to be $85 \%$ by HPLC analysis on a Daicel Chiralpak IF column with a gradient 90:5:5 Hexane/EtOH/DCM, flow rate $0.8 \mathrm{~mL} / \mathrm{min}, \lambda=220 \mathrm{~nm}$ : $t_{r_{\text {major }}}=15.4 \mathrm{~min}, t_{r \text { minor }}=17,1 \mathrm{~min}$. Experimental $[\alpha]_{\mathrm{D}}{ }^{25}=-24.0\left(\mathrm{c}=0.25, \mathrm{CH}_{2} \mathrm{Cl}_{2}\right) .{ }^{1} \mathrm{H}$ $\operatorname{NMR}\left(\mathrm{CDCl}_{3}, 400 \mathrm{MHz}\right) \delta: 3.67(\mathrm{t}, 4 \mathrm{H}, \mathrm{J}=4.7 \mathrm{~Hz}), 3.36(\mathrm{~m}, 4 \mathrm{H}), 2.74(\mathrm{~m}, 1 \mathrm{H}), 2.44(\mathrm{~m}$, 2H), $2.35(\mathrm{~m}, 2 \mathrm{H}), 2.27(\mathrm{~m}, 2 \mathrm{H}), 1.91(\mathrm{~m}, 1 \mathrm{H}), 1.52(\mathrm{~m}, 1 \mathrm{H}), 1.19(\mathrm{t}, 3 \mathrm{H}, J=7.1 \mathrm{~Hz}), 1.11$ $(\mathrm{d}, 3 \mathrm{H}, J=6.8 \mathrm{~Hz}), 1.09(\mathrm{t}, 3 \mathrm{H}, J=7.1 \mathrm{~Hz}) \cdot{ }^{13} \mathrm{C}\left\{{ }^{1} \mathrm{H}\right\} \mathrm{NMR}\left(\mathrm{CDCl}_{3}, 100.6 \mathrm{MHz}\right) \delta: 175.7$, $67.2,56.9,53.8,42.0,40.6,33.6,31.2,18.7,15.1$, 13.3. HRMS (ESI-TOF) m/z: [M + H]+ Calcd for $\mathrm{C}_{13} \mathrm{H}_{27} \mathrm{~N}_{2} \mathrm{O}_{2}$ 243.2073; Found 243.2080.

\section{tert-butyl 4-(4-(diethylamino)-3-methyl-4-oxobutyl)piperazine-1 carboxylate (4b).}

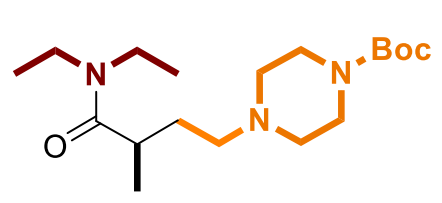

chromatography on silica eluting with $\mathrm{DCM} / \mathrm{MeOH}$ (96:4) afforded $4 \mathrm{~b}$ (124.3 mg, 73\%) as yellow oil. The enantiomeric excess was determined to be $84 \%$ by HPLC analysis on a Daicel Chiralpak ID column with a gradient 85:15 Hexane/lsopropanol, flow rate 1 $\mathrm{mL} / \mathrm{min}, \lambda=220 \mathrm{~nm}: t_{r \text { minor }}=11.9 \mathrm{~min}, t_{r \text { major }}=13.4 \mathrm{~min}$. Experimental $[\alpha]_{\mathrm{D}}^{25}=-19.5(\mathrm{c}$ $\left.=0.22, \mathrm{CH}_{2} \mathrm{Cl}_{2}\right) .{ }^{1} \mathrm{H}$ NMR $\left(\mathrm{CDCl}_{3}, 400 \mathrm{MHz}\right) \delta: 3.37(\mathrm{~m}, 8 \mathrm{H}), 2.74(\mathrm{~m}, 1 \mathrm{H}), 2.32(\mathrm{~m}, 6 \mathrm{H})$, $1.91(\mathrm{~m}, 1 \mathrm{H}), 1.53(\mathrm{~m}, 1 \mathrm{H}), 1.45(\mathrm{~s}, 9 \mathrm{H}), 1.18(\mathrm{t}, 3 \mathrm{H}, J=7.1 \mathrm{~Hz}), 1.11(\mathrm{~d}, 3 \mathrm{H}, J=6.8 \mathrm{~Hz})$, $1.09(\mathrm{t}, 3 \mathrm{H}, J=7.1 \mathrm{~Hz}) .{ }^{13} \mathrm{C}\left\{{ }^{1} \mathrm{H}\right\} \operatorname{NMR}\left(\mathrm{CDCl}_{3}, 100.6 \mathrm{MHz}\right) \delta: 175.7,155.0,79.7,56.4$, 53.1, 42.0, 40.6, 33.6, 31.4, 28.6, 18.7, 15.1, 13.3. HRMS (ESI-TOF) m/z: [M + H]+ Calcd for $\mathrm{C}_{18} \mathrm{H}_{36} \mathrm{~N}_{3} \mathrm{O}_{3} 342.2757$; Found 342.2766.

\section{4-(benzyl(methyl)amino)-N,N-diethyl-2-methylbutanamide (4c).}

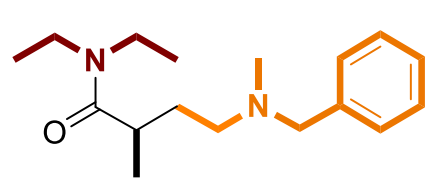

General procedure E was followed by employing N,Ndiethylmethacrylamide $1 \mathrm{a}(78.5 \mu \mathrm{L}, 0.5 \mathrm{mmol})$ and $\mathrm{N}$ Benzylmethylamine $(64.5 \mu \mathrm{L}, 0.5 \mathrm{mmol})$. Purification by flash chromatography on silica eluting with $\mathrm{DCM} / \mathrm{MeOH}$ (96:4) afforded 4c (102.4 mg, 74\%) as yellow oil. The enantiomeric excess was determined to be $82 \%$ by HPLC analysis on a Daicel Chiralpak IC column with a gradient 80:15:05 tert-Butyl methyl ether $/ \mathrm{Hexane} / \mathrm{EtOH}$, flow rate $0.8 \mathrm{~mL} / \mathrm{min}, \lambda=230 \mathrm{~nm}: t_{r \text { minor }}=9.6 \mathrm{~min}, t_{r \text { major }}=10.3 \mathrm{~min}$. Experimental $[\alpha]_{\mathrm{D}}{ }^{25}=-29.5\left(\mathrm{c}=0.31, \mathrm{CH}_{2} \mathrm{Cl}_{2}\right) .{ }^{1} \mathrm{H} \mathrm{NMR}\left(\mathrm{CDCl}_{3}, 400 \mathrm{MHz}\right) \delta: 7.27-7.33$ $(\mathrm{m}, 4 \mathrm{H}), 7.21-7.26(\mathrm{~m}, 1 \mathrm{H}), 3.46(\mathrm{~m}, 4 \mathrm{H}), 3.27(\mathrm{~m}, 2 \mathrm{H}), 2.79(\mathrm{~m}, 1 \mathrm{H}), 2.36(\mathrm{~m}, 2 \mathrm{H})$, 
$2.15(\mathrm{~s}, 3 \mathrm{H}), 1.94(\mathrm{~m}, 1 \mathrm{H}), 1.58(\mathrm{~m}, 1 \mathrm{H}), 1.17(\mathrm{t}, 3 \mathrm{H}, J=7.1 \mathrm{~Hz}), 1.09(\mathrm{~d}, 3 \mathrm{H}, J=6.8 \mathrm{~Hz})$, $1.09(\mathrm{t}, 3 \mathrm{H}, J=7.1 \mathrm{~Hz}) .{ }^{13} \mathrm{C}\left\{{ }^{1} \mathrm{H}\right\} \mathrm{NMR}\left(\mathrm{CDCl}_{3}, 100.6 \mathrm{MHz}\right) \delta: 176.0,129.2,128.3,127.1$, 62.3, 55.4, 42.2, 41.9, 40.5, 33.4, 32.1, 18.4, 15.1, 13.3. HRMS (ESI-TOF) m/z: [M + H]+ Calcd for $\mathrm{C}_{17} \mathrm{H}_{29} \mathrm{~N}_{2} \mathrm{O} 277.2280$; Found 277.2292.

\section{N,N-diethyl-4-(4-(2-methoxyphenyl)piperazin-1-yl)-2-methylbutanamide (4d).}

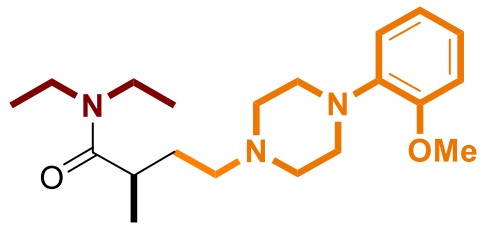

General procedure E was followed by employing N,Ndiethylmethacrylamide $1 \mathrm{a}(78.5 \mu \mathrm{L}, 0.5 \mathrm{mmol})$ and 1-(2methoxyphenyl)piperazine (96.1 $\mathrm{mg}, \quad 0.5 \mathrm{mmol})$. Purification by flash chromatography on silica eluting with $\mathrm{DCM} / \mathrm{MeOH}(98: 2)$ to afforded $4 \mathbf{d}(83.5 \mathrm{mg}, 48 \%)$ as colourless oil. The enantiomeric excess was determined to be $82 \%$ by HPLC analysis on a Daicel Chiralpak ID column with a gradient 70:30 Hexane/lsopropanol, flow rate 1 $\mathrm{mL} / \mathrm{min}, \lambda=230 \mathrm{~nm}: t_{r \text { minor }}=7.3 \mathrm{~min}, t_{r \text { major }}=8.4 \mathrm{~min}$. Experimental $[\alpha]_{\mathrm{D}}{ }^{25}=-21.0(\mathrm{c}=$ $\left.0.40, \mathrm{CH}_{2} \mathrm{Cl}_{2}\right) .{ }^{1} \mathrm{H}$ NMR $\left(\mathrm{CDCl}_{3}, 400 \mathrm{MHz}\right) \delta: 6.99(\mathrm{~m}, 1 \mathrm{H}), 6.92(\mathrm{~m}, 2 \mathrm{H}), 6.86(\mathrm{~m}, 1 \mathrm{H})$, $3.85(\mathrm{~s}, 3 \mathrm{H}), 3.46(\mathrm{~m}, 2 \mathrm{H}), 3.31(\mathrm{~m}, 2 \mathrm{H}), 3.07(\mathrm{bs}, 4 \mathrm{H}), 2.77(\mathrm{~m}, 1 \mathrm{H}), 2.58(\mathrm{bs}, 4 \mathrm{H}), 2.35$ $(\mathrm{m}, 2 \mathrm{H}), 1.93(\mathrm{~m}, 1 \mathrm{H}), 1.60(\mathrm{~m}, 1 \mathrm{H}), 1.19(\mathrm{t}, 3 \mathrm{H}, J=7.1 \mathrm{~Hz}), 1.12(\mathrm{~m}, 6 \mathrm{H}) .{ }^{13} \mathrm{C}\left\{{ }^{1} \mathrm{H}\right\} \mathrm{NMR}$ $\left(\mathrm{CDCl}_{3}, 100.6 \mathrm{MHz}\right) \delta: 13.3,15.1,18.7,31.6,33.6,40.6,42.0,50.8,53.6,55.5,56.4$, 111.4, 118.3, 121.1, 123.0, 141.6, 152.4, 175.8. HRMS (ESI-TOF) m/z: [M + H]+ Calcd for $\mathrm{C}_{20} \mathrm{H}_{34} \mathrm{~N}_{3} \mathrm{O}_{2}$ 348.2651; Found 348.2660.

\section{N,N-diethyl-2-methyl-4-(phenylamino)butanamide (4e).}

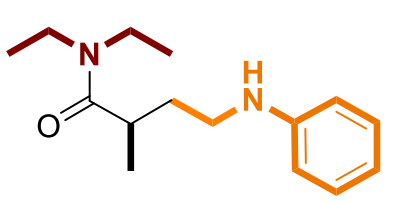

General procedure E was followed by employing N,Ndiethylmethacrylamide $1 \mathrm{a}(78.5 \mu \mathrm{L}, 0.5 \mathrm{mmol})$ and aniline (45.6 $\mu \mathrm{L}, 0.5 \mathrm{mmol})$. Purification by flash chromatography on silica eluting with PE/EtOAc (8:2) to afforded $4 \mathrm{e}(69.6 \mathrm{mg}, 56 \%)$ as orange oil. The enantiomeric excess was determined to be $78 \%$ by HPLC analysis on a Daicel Chiralpak ID column with a gradient 90:10 Hexane/Isopropanol, flow rate 1 $\mathrm{mL} / \mathrm{min}, \lambda=230 \mathrm{~nm}: t_{r \text { minor }}=16.2 \mathrm{~min}, t_{r \text { major }}=17.1 \mathrm{~min}$. Experimental $[\alpha]_{\mathrm{D}}^{25}=-23.6(\mathrm{c}$ $\left.=0.33, \mathrm{CH}_{2} \mathrm{Cl}_{2}\right) .{ }^{1} \mathrm{H} \mathrm{NMR}\left(\mathrm{CDCl}_{3}, 400 \mathrm{MHz}\right) \delta: 7.15(\mathrm{~m}, 2 \mathrm{H}), 6.67(\mathrm{tt}, 1 \mathrm{H}, \mathrm{J}=1.0 \mathrm{~Hz}, \mathrm{~J}=$ $7.3 \mathrm{~Hz}), 6.56(\mathrm{~m}, 2 \mathrm{H}), 3.69$ (bs, $1 \mathrm{H}), 3.37$ (dq, $2 \mathrm{H}, J=1.4 \mathrm{~Hz}, J=7.1 \mathrm{~Hz}), 3.29(\mathrm{~m}, 2 \mathrm{H})$, $3.09(\mathrm{~m}, 2 \mathrm{H}), 2.78(\mathrm{~m}, 1 \mathrm{H}), 2.11(\mathrm{~m}, 1 \mathrm{H}), 1.69(\mathrm{~m}, 1 \mathrm{H}), 1.16(\mathrm{~d}, 3 \mathrm{H}, J=6.8 \mathrm{~Hz}), 1.11(\mathrm{q}$, $6 \mathrm{H}, J=7.2 \mathrm{~Hz}) .{ }^{13} \mathrm{C}\left\{{ }^{1} \mathrm{H}\right\} \mathrm{NMR}\left(\mathrm{CDCl}_{3}, 100.6 \mathrm{MHz}\right) \delta: 175.7,148.6,129.3,117.3,112.8$, 
42.5, 42.0, 40.7, 34.1, 34.0, 18.7, 15.0, 13.3. HRMS (ESI-TOF) m/z: [M + H]+ Calcd for $\mathrm{C}_{15} \mathrm{H}_{25} \mathrm{~N}_{2} \mathrm{O} 249.1967 ;$ Found 249.1968.

N,N-diisopropyl-2-methyl-4-morpholinobutanamide (4f).

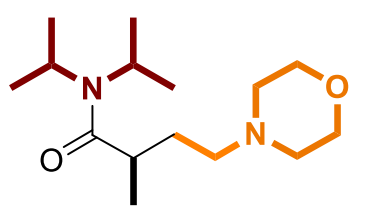

General procedure E was followed by employing N,N-diisopropyl2-methyl-4-oxobutanamide 1c (84.4 $\mathrm{mg}, 0.5 \mathrm{mmol})$ and morpholine (43.7 $\mu \mathrm{L}, \quad 0.5 \mathrm{mmol})$. Purification by flash chromatography on silica eluting with DCM/MeOH (96/4) afforded $4 f(90.6 \mathrm{mg}, 67 \%)$ as orange oil. The enantiomeric excess was determined to be $84 \%$ by HPLC analysis on a Daicel Chiralpak IF column with a gradient 90:10 Hexane/Ethanol, flow rate $1 \mathrm{~mL} / \mathrm{min}, \lambda=220 \mathrm{~nm}: t_{r \text { major }}=8.8 \mathrm{~min}, t_{r \text { minor }}=9.8 \mathrm{~min}$. Experimental $[\alpha]_{\mathrm{D}}{ }^{25}=-23.3\left(\mathrm{c}=0.30, \mathrm{CH}_{2} \mathrm{Cl}_{2}\right) .{ }^{1} \mathrm{H} \mathrm{NMR}\left(\mathrm{CDCl}_{3}, 400 \mathrm{MHz}\right) \delta: 4.07(\mathrm{~m}$, 1H), 3.68 (t, 4H, J = 4.7 Hz), $3.50(\mathrm{bs}, 1 \mathrm{H}), 2.71(\mathrm{~m}, 1 \mathrm{H}), 2.38(\mathrm{~m}, 6 \mathrm{H}), 1.90(\mathrm{~m}, 1 \mathrm{H})$, $1.49(\mathrm{~m}, 1 \mathrm{H}), 1.35(\mathrm{~m}, 6 \mathrm{H}), 1.21(\mathrm{~m}, 6 \mathrm{H}), 1.08(\mathrm{~d}, 3 \mathrm{H}, J=6.8 \mathrm{~Hz}) .{ }^{13} \mathrm{C}\left\{{ }^{1} \mathrm{H}\right\} \mathrm{NMR}\left(\mathrm{CDCl}_{3}\right.$, 100.6 MHz) $\delta: 175.4,67.2,56.9,53.9,47.9,45.8,34.9,31.0,21.5,20.9,20.9,18.5$. HRMS (ESI-TOF) m/z: [M + H]+ Calcd for $\mathrm{C}_{15} \mathrm{H}_{31} \mathrm{~N}_{2} \mathrm{O}_{2}$ 271.2386; Found 271.2389.

\section{2-methyl-4-morpholino-N,N-diphenylbutanamide (4g).}

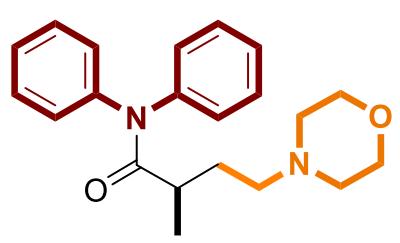

General procedure E was followed by employing N,Ndiphenylmethacrylamide $1 \mathrm{~b}$ (118.7 $\mathrm{mg}, 0.5 \mathrm{mmol})$ and morpholine $(43.7 \mu \mathrm{L}, 0.5 \mathrm{mmol})$. Purification by flash chromatography on silica eluting with $\mathrm{DCM} / \mathrm{MeOH}(98 / 2)$ afforded $\mathbf{4 g}$ (96.6 $\mathrm{mg}, 57 \%)$ as colourless oil. The enantiomeric excess was determined to be $70 \%$ by HPLC analysis on a Daicel Chiralpak IA column with a gradient 80:20 Hexane/lsopropanol, flow rate $1 \mathrm{~mL} / \mathrm{min}, \lambda=230 \mathrm{~nm}: t_{r \text { minor }}=6.8 \mathrm{~min}, t_{\text {major }}=7.7 \mathrm{~min}$. Experimental $[\alpha]_{D}{ }^{25}=-10.3\left(\mathrm{c}=0.30, \mathrm{CH}_{2} \mathrm{Cl}_{2}\right) .{ }^{1} \mathrm{H}$ NMR $\left(\mathrm{CDCl}_{3}, 400 \mathrm{MHz}\right) \delta: 7.33$ (bs, $10 \mathrm{H}), 3.65(\mathrm{t}, 4 \mathrm{H}, J=4.7 \mathrm{~Hz}), 2.69(\mathrm{~m}, 1 \mathrm{H}), 2.35(\mathrm{~m}, 6 \mathrm{H}), 2.01(\mathrm{~m}, 1 \mathrm{H}), 1.48(\mathrm{~m}, 1 \mathrm{H})$, $1.13(\mathrm{~d}, 3 \mathrm{H}, J=6.8 \mathrm{~Hz}) .{ }^{13} \mathrm{C}\left\{{ }^{1} \mathrm{H}\right\} \mathrm{NMR}\left(\mathrm{CDCl}_{3}, 100.6 \mathrm{MHz}\right) \delta: 176.8,143.2,126.0-129.9$, 67.1, 56.7, 53.8, 35.6, 30.9, 18.4. HRMS (ESI-TOF) m/z: [M + H]+ Calcd for $\mathrm{C}_{21} \mathrm{H}_{27} \mathrm{~N}_{2} \mathrm{O}_{2}$ 339.2073; Found 339.2077.

\section{N,N,2-triethyl-4-morpholinobutanamide (4h).}

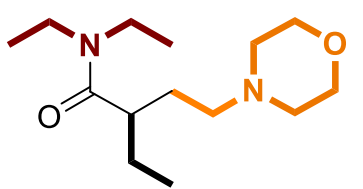

General procedure E was followed by employing N,N-diethyl-2methylenebutanamide 1d $(77.5 \mathrm{mg}, 0.5 \mathrm{mmol})$ and morpholine

$(43.7 \mu \mathrm{L}, 0.5 \mathrm{mmol})$. Purification by flash chromatography eluting with $\mathrm{DCM} / \mathrm{MeOH}(98 / 2)$ afforded $\mathbf{4 h}(99.8 \mathrm{mg}, 78 \%)$ as colourless oil. The ee was determined to be $84 \%$ by HPLC analysis on a Daicel Chiralpak IC column with a gradient 
80:20 Hexane/lsopropanol, flow rate $1 \mathrm{~mL} / \mathrm{min}, \lambda=230 \mathrm{~nm}: t_{r \text { major }}=17.3 \mathrm{~min}, t_{r \text { minor }}=$ 19.9 min. Experimental $[\alpha]_{D}{ }^{25}=-12.9\left(\mathrm{c}=0.44, \mathrm{CH}_{2} \mathrm{Cl}_{2}\right) .{ }^{1} \mathrm{H} \mathrm{NMR}\left(\mathrm{CDCl}_{3}, 400 \mathrm{MHz}\right) \delta$ : $3.68(\mathrm{t}, 4 \mathrm{H}, J=4.7 \mathrm{~Hz}), 3.49(\mathrm{~m}, 1 \mathrm{H}), 3.33(\mathrm{~m}, 3 \mathrm{H}), 2.60(\mathrm{~m}, 1 \mathrm{H}), 2.21-2.46(\mathrm{~m}, 6 \mathrm{H})$, $1.84(\mathrm{~m}, 1 \mathrm{H}), 1.64(\mathrm{~m}, 2 \mathrm{H}), 1.47(\mathrm{~m}, 1 \mathrm{H}), 1.19(\mathrm{t}, 3 \mathrm{H}, J=7.2 \mathrm{~Hz}), 1.11(\mathrm{t}, 3 \mathrm{H}, J=7.1 \mathrm{~Hz})$, $0.89(\mathrm{t}, 3 \mathrm{H}, J=7.4 \mathrm{~Hz}) \cdot{ }^{13} \mathrm{C}\left\{{ }^{1} \mathrm{H}\right\}$ NMR $\left(\mathrm{CDCl}_{3}, 100.6 \mathrm{MHz}\right) \delta: 175.1,67.2,56.9,53.8$, 42.0, 40.6, 40.6, 29.5, 26.7, 15.1, 13.3, 12.2. HRMS (ESI-TOF) m/z: [M + H]+ Calcd for $\mathrm{C}_{14} \mathrm{H}_{29} \mathrm{~N}_{2} \mathrm{O}_{2} 257.2229$; Found 257.2247.

\section{2-benzyl-N,N-diethyl-4-morpholinobutanamide (4i).}

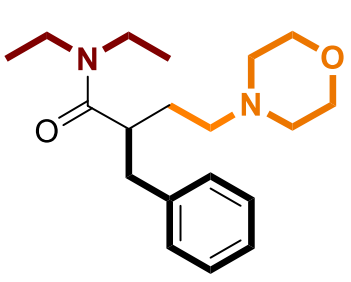

General procedure E was followed by employing 2-benzyl-N,N-

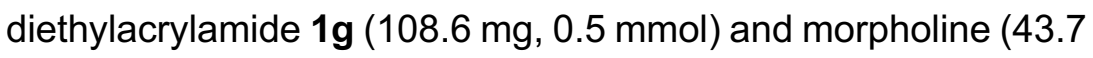
$\mu \mathrm{L}, 0.5 \mathrm{mmol})$. Purification by flash chromatography eluting on silica with $\mathrm{DCM} / \mathrm{MeOH}(94: 6)$ afforded $4 \mathbf{i}(119.1 \mathrm{mg}, 75 \%)$ as yellow oil. The ee was determined to be $86 \%$ by HPLC analysis on a Daicel Chiralpak IC column with a gradient 95:05 tert-Butyl methyl ether/Ethanol, flow rate $1 \mathrm{~mL} / \mathrm{min}, \lambda=220 \mathrm{~nm}: t_{r \text { major }}=8.5 \mathrm{~min}, t_{r \text { minor }}=10.6 \mathrm{~min}$. Experimental $[\alpha]_{D}{ }^{25}=$ $+11.8\left(\mathrm{c}=0.40, \mathrm{CH}_{2} \mathrm{Cl}_{2}\right) .{ }^{1} \mathrm{H} \mathrm{NMR}\left(\mathrm{CDCl}_{3}, 400 \mathrm{MHz}\right) \delta: 7.21-7.26(\mathrm{~m}, 2 \mathrm{H}), 7.14-7.19$ $(\mathrm{m}, 3 \mathrm{H}), 3.65(\mathrm{t}, 4 \mathrm{H}, J=4.7 \mathrm{~Hz}), 3.36(\mathrm{~m}, 1 \mathrm{H}), 3.21(\mathrm{~m}, 2 \mathrm{H}), 2.96(\mathrm{~m}, 3 \mathrm{H}), 2.67(\mathrm{~m}, 1 \mathrm{H})$, $2.30(\mathrm{~m}, 6 \mathrm{H}), 1.95(\mathrm{~m}, 1 \mathrm{H}), 1.64(\mathrm{~m}, 1 \mathrm{H}), 1.03(\mathrm{t}, 3 \mathrm{H}, J=7.1 \mathrm{~Hz}), 0.89(\mathrm{t}, 3 \mathrm{H}, J=7.1 \mathrm{~Hz})$. ${ }^{13} \mathrm{C}\left\{{ }^{1} \mathrm{H}\right\}$ NMR $\left(\mathrm{CDCl}_{3}, 100.6 \mathrm{MHz}\right) \delta: 174.3,140.1,129.3,128.4,126.4,67.2,56.7,53.7$, 41.7, 41.7, 40.7, 40.2, 29.7, 14.5, 13.2. HRMS (ESI-TOF) m/z: [M + H]+ Calcd for $\mathrm{C}_{19} \mathrm{H}_{31} \mathrm{~N}_{2} \mathrm{O}_{2}$ 319.2386; Found 319.2386.

\section{N,N-diethyl-4-morpholino-2-phenylbutanamide (4j).}

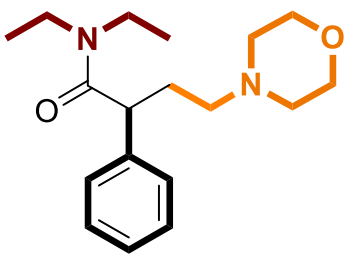

General procedure E was followed by employing N,N-diethyl-2phenylacrylamide $1 \mathrm{~h}(50.8 \mathrm{mg}, 0.25 \mathrm{mmol})$ and morpholine (21.9 $\mu \mathrm{L}, 0.25 \mathrm{mmol})$. Purification by flash chromatography on florisil eluting with $\mathrm{DCM} / \mathrm{MeOH}(97: 3)$ afforded $4 \mathrm{j}$ (49.4 $\mathrm{mg}, 65 \%)$ as orange oil. The ee was determined to be $78 \%$ by HPLC analysis on a Daicel Chiralpak ID column with a gradient 90:10 Hexane/lsopropanol, flow rate $1 \mathrm{~mL} / \mathrm{min}, \lambda=210 \mathrm{~nm}: t_{r}$ major $=9.6 \mathrm{~min}, t_{r \text { minor }}=10.9 \mathrm{~min}$. Experimental $[\alpha]_{D}{ }^{25}=+36.1\left(\mathrm{c}=0.33, \mathrm{CH}_{2} \mathrm{Cl}_{2}\right) .{ }^{1} \mathrm{H} \mathrm{NMR}$ $\left(\mathrm{CDCl}_{3}, 400 \mathrm{MHz}\right) \delta: 7.20-7.31(\mathrm{~m}, 5 \mathrm{H}), 3.87(\mathrm{~m}, 1 \mathrm{H}), 3.70(\mathrm{t}, 4 \mathrm{H}, J=4.6 \mathrm{~Hz}), 3.47(\mathrm{~m}$, $1 \mathrm{H}), 3.35(\mathrm{~m}, 1 \mathrm{H}), 3.25(\mathrm{~m}, 1 \mathrm{H}), 3.15(\mathrm{~m}, 1 \mathrm{H}), 2.41(\mathrm{~m}, 4 \mathrm{H}), 2.29(\mathrm{~m}, 3 \mathrm{H}), 1.82(\mathrm{~m}, 1 \mathrm{H})$, $1.06(\mathrm{~m}, 6 \mathrm{H}) .{ }^{13} \mathrm{C}\left\{{ }^{1} \mathrm{H}\right\} \mathrm{NMR}\left(\mathrm{CDCl}_{3}, 100.6 \mathrm{MHz}\right) \delta: 172.0,140.9,128.8,128.0,126.9$, 67.3, 56.7, 53.8, 46.3, 41.9, 40.6, 32.2, 14.5, 13.1. HRMS (ESI-TOF) m/z: [M + H]+ Calcd for $\mathrm{C}_{18} \mathrm{H}_{29} \mathrm{~N}_{2} \mathrm{O}_{2}$ 305.2229; Found 305.2224. 


\section{1-(azepan-1-yl)-4-morpholino-2-phenylbutan-1-one (4k).}

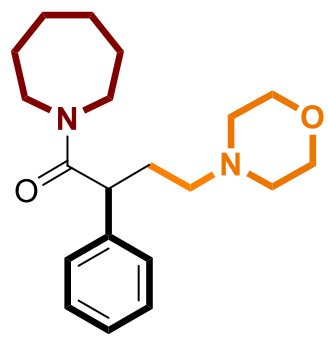

General procedure E was followed by employing 1-(azepan-1-yl)2-phenylprop-2-en-1-one 1i (57.4 mg, $0.25 \mathrm{mmol}$ ) and morpholine $(21.9 \mu \mathrm{L}, 0.25 \mathrm{mmol})$. Purification by flash chromatography on silica eluting with $\mathrm{DCM} / \mathrm{MeOH}$ (95:5) afforded $\mathbf{4 k}$ (48.8 mg, 59\%) as orange oil. The ee was determined to be $84 \%$ by HPLC analysis on a Daicel Chiralpak IC column with a gradient 80:20

Hexane/lsopropanol, flow rate $1 \mathrm{~mL} / \mathrm{min}, \lambda=220 \mathrm{~nm}: t_{r \text { major }}=17.4 \mathrm{~min}, t_{r \text { minor }}=24.6 \mathrm{~min}$. Experimental $[\alpha]_{D}{ }^{25}=+39.1\left(\mathrm{c}=0.35, \mathrm{CH}_{2} \mathrm{Cl}_{2}\right){ }^{1} \mathrm{H} \mathrm{NMR}\left(\mathrm{CDCl}_{3}, 400 \mathrm{MHz}\right) \delta: 7.27-7.32$ (m, 4H), $7.19-7.25(\mathrm{~m}, 1 \mathrm{H}), 3.90(\mathrm{~m}, 1 \mathrm{H}), 3.70(\mathrm{~m}, 5 \mathrm{H}), 3.54(\mathrm{~m}, 1 \mathrm{H}), 2.41(\mathrm{~m}, 4 \mathrm{H})$, $2.30(\mathrm{~m}, 3 \mathrm{H}), 1.84(\mathrm{~m}, 1 \mathrm{H}), 1.25-1.77(\mathrm{~m}, 8 \mathrm{H}) \cdot{ }^{13} \mathrm{C}\left\{{ }^{1} \mathrm{H}\right\} \mathrm{NMR}\left(\mathrm{CDCl}_{3}, 100.6 \mathrm{MHz}\right) \delta$ : 172.4, 140.8, 128.8, 128.1, 127.0, 67.3, 56.7, 53.8, 47.8, 46.5, 46.4, 32.1, 29.3, 27.6, 27.0, 26.7. HRMS (ESI-TOF) m/z: [M + H]+ Calcd for $\mathrm{C}_{20} \mathrm{H}_{31} \mathrm{~N}_{2} \mathrm{O}_{2}$ 331.2386; Found 331.2390 .

\section{1-(azepan-1-yl)-4-(4-(2-methoxyphenyl)piperazin-1-yl)-2-phenylbutan-1-one (4I). ${ }^{11}$}

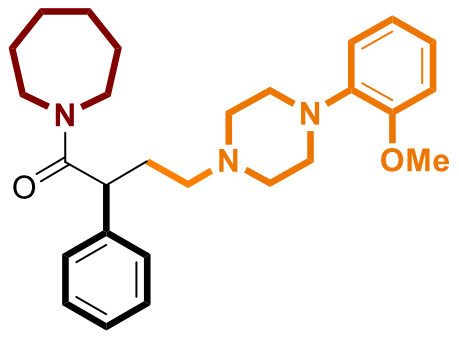

General procedure E was followed by employing 1(azepan-1-yl)-2-phenylprop-2-en-1-one 1i (57.4 mg, 0.25 $\mathrm{mmol}$ ) and 1-(2-methoxyphenyl)piperazine (48.1 mg, 0.25 mmol). Purification by flash chromatography on silica eluting with DCM/MeOH (98:2) afforded 4I (69.6 mg, 64\%) as colourless oil. The ee was determined to be $82 \%$ by HPLC analysis on a Daicel Chiralpak IF column with a gradient 80:20 Hexane/isopropanol with $0.2 \%$ of diethylamine as additive, flow rate $1 \mathrm{~mL} / \mathrm{min}, \lambda=220$ $\mathrm{nm}: t_{r \text { major }}=8.5 \mathrm{~min}, t_{r \text { minor }}=9.5 \mathrm{~min}$. Experimental $[\mathrm{\alpha}]_{\mathrm{D}}{ }^{25}=+31.0\left(\mathrm{c}=0.63, \mathrm{CH}_{2} \mathrm{Cl}_{2}\right) .{ }^{1} \mathrm{H}$ $\operatorname{NMR}\left(\mathrm{CDCl}_{3}, 400 \mathrm{MHz}\right) \delta: 7.20-7.33(\mathrm{~m}, 5 \mathrm{H}), 6.84-7.01(\mathrm{~m}, 4 \mathrm{H}), 3.94(\mathrm{t}, 1 \mathrm{H}, J=7.6$ $\mathrm{Hz}), 3.85(\mathrm{~s}, 3 \mathrm{H}), 3.71(\mathrm{~m}, 1 \mathrm{H}), 3.55(\mathrm{~m}, 1 \mathrm{H}), 3.29(\mathrm{~m}, 2 \mathrm{H}), 3.09(\mathrm{bs}, 4 \mathrm{H}), 2.62(\mathrm{bs}, 4 \mathrm{H})$, $2.37(\mathrm{~m}, 3 \mathrm{H}), 1.87(\mathrm{~m}, 1 \mathrm{H}), 1.25-1.78(\mathrm{~m}, 8 \mathrm{H}) .{ }^{13} \mathrm{C}\left\{{ }^{1} \mathrm{H}\right\} \mathrm{NMR}\left(\mathrm{CDCl}_{3}, 100.6 \mathrm{MHz}\right) \delta$ : $172.5,152.4,141.6,140.9,128.8,128.1$, 126.9, 122.9, 121.1, 118.2, 111.4, 56.3, 55.5, 53.4, 50.9, 47.9, 46.5, 46.4, 32.4, 29.3, 27.6, 27.0, 26.7. HRMS (ESI-TOF) m/z: [M + H]+ Calcd for $\mathrm{C}_{27} \mathrm{H}_{38} \mathrm{~N}_{3} \mathrm{O}_{2}$ 436.2964; Found 436.2967. These signals are in agreement with those reported in the literature. 
SXVIII. Spectroscopic data

a. $\alpha$-Alkyl acrylate acids ${ }^{1} \mathrm{H} \mathrm{NMR}\left(\mathrm{CDCl}_{3}, 400 \mathrm{MHz}\right)$ 


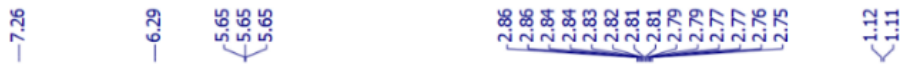<smiles>C=C(C(=O)O)C(C)C</smiles>

$11 \mathrm{a}$

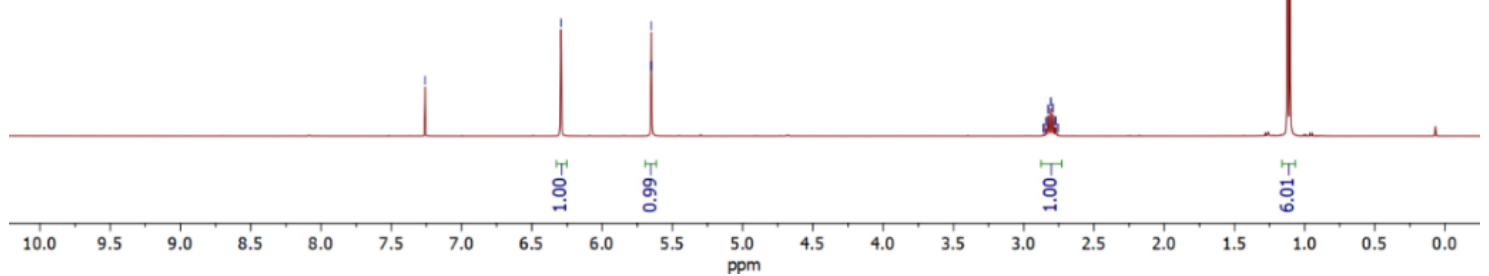

${ }^{13} \mathrm{C}\left\{{ }^{1} \mathrm{H}\right\} \mathrm{NMR}\left(\mathrm{CDCl}_{3}, 100.6 \mathrm{MHz}\right)$

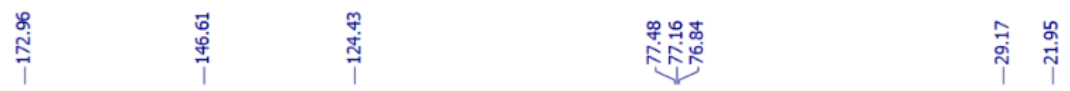<smiles>C=C(C(=O)O)C(C)C</smiles>

$11 \mathrm{a}$

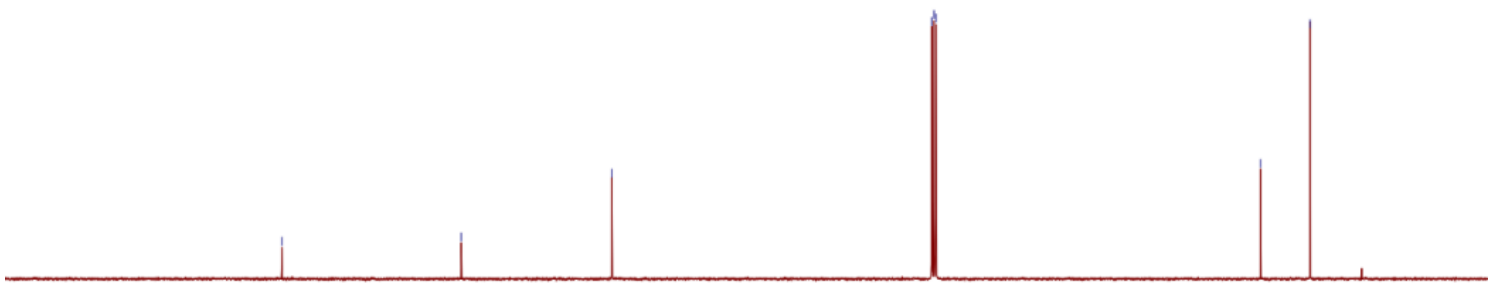

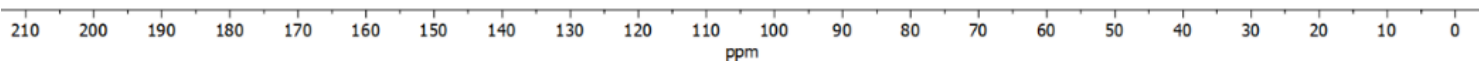

${ }^{1} \mathrm{H}$ NMR $\left(\mathrm{CDCl}_{3}, 400 \mathrm{MHz}\right)$ 
<smiles>C=C(CC)C(=O)O</smiles>

11b

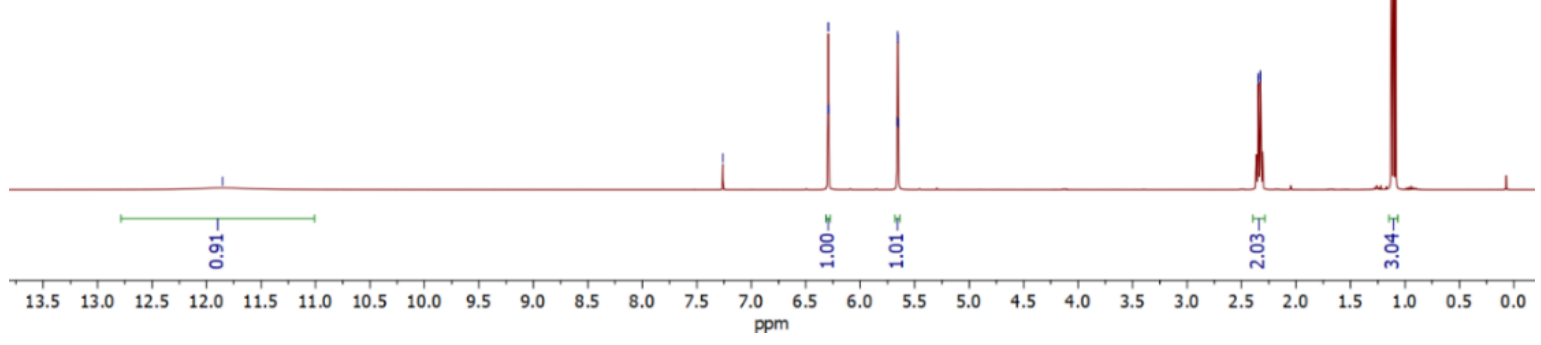

${ }^{13} \mathrm{C}\left\{{ }^{1} \mathrm{H}\right\} \operatorname{NMR}\left(\mathrm{CDCl}_{3}, 100.6 \mathrm{MHz}\right)$

焉<smiles>C=C(CC)C(=O)O</smiles>

$11 \mathrm{~b}$

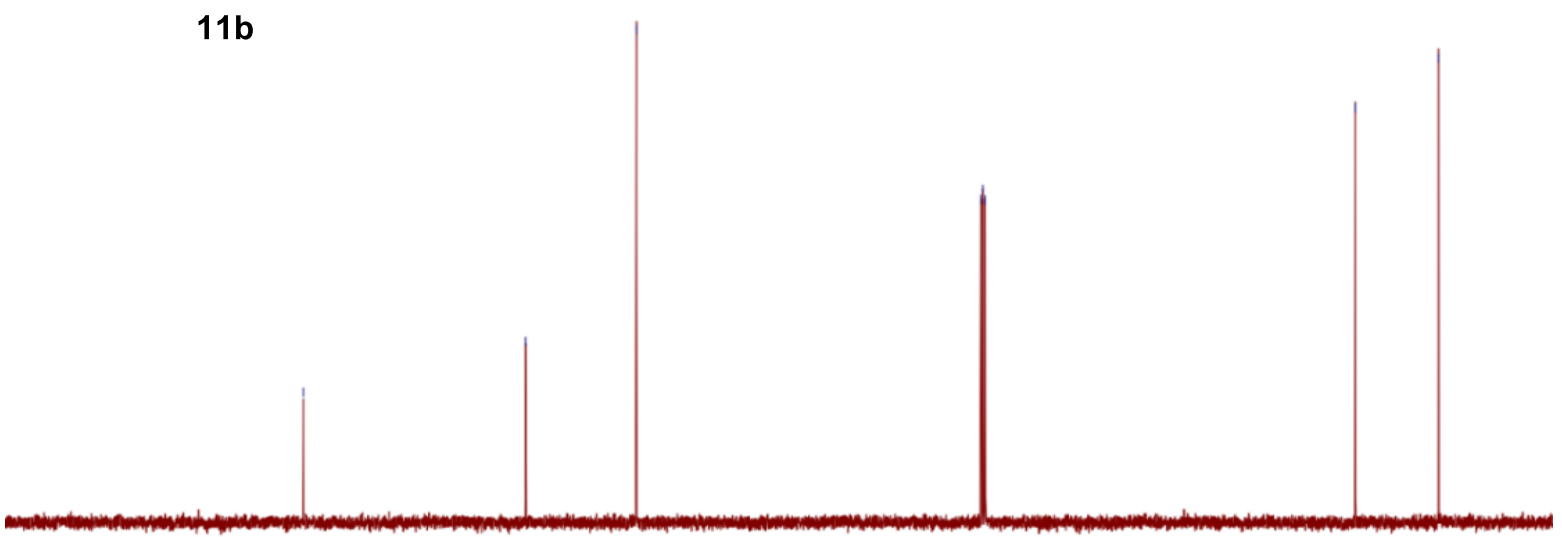

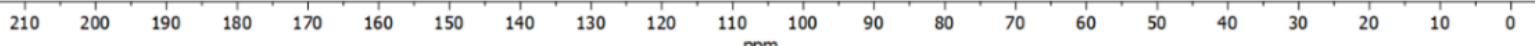

${ }^{1} \mathrm{H} \mathrm{NMR}\left(\mathrm{CDCl}_{3}, 400 \mathrm{MHz}\right)$ 


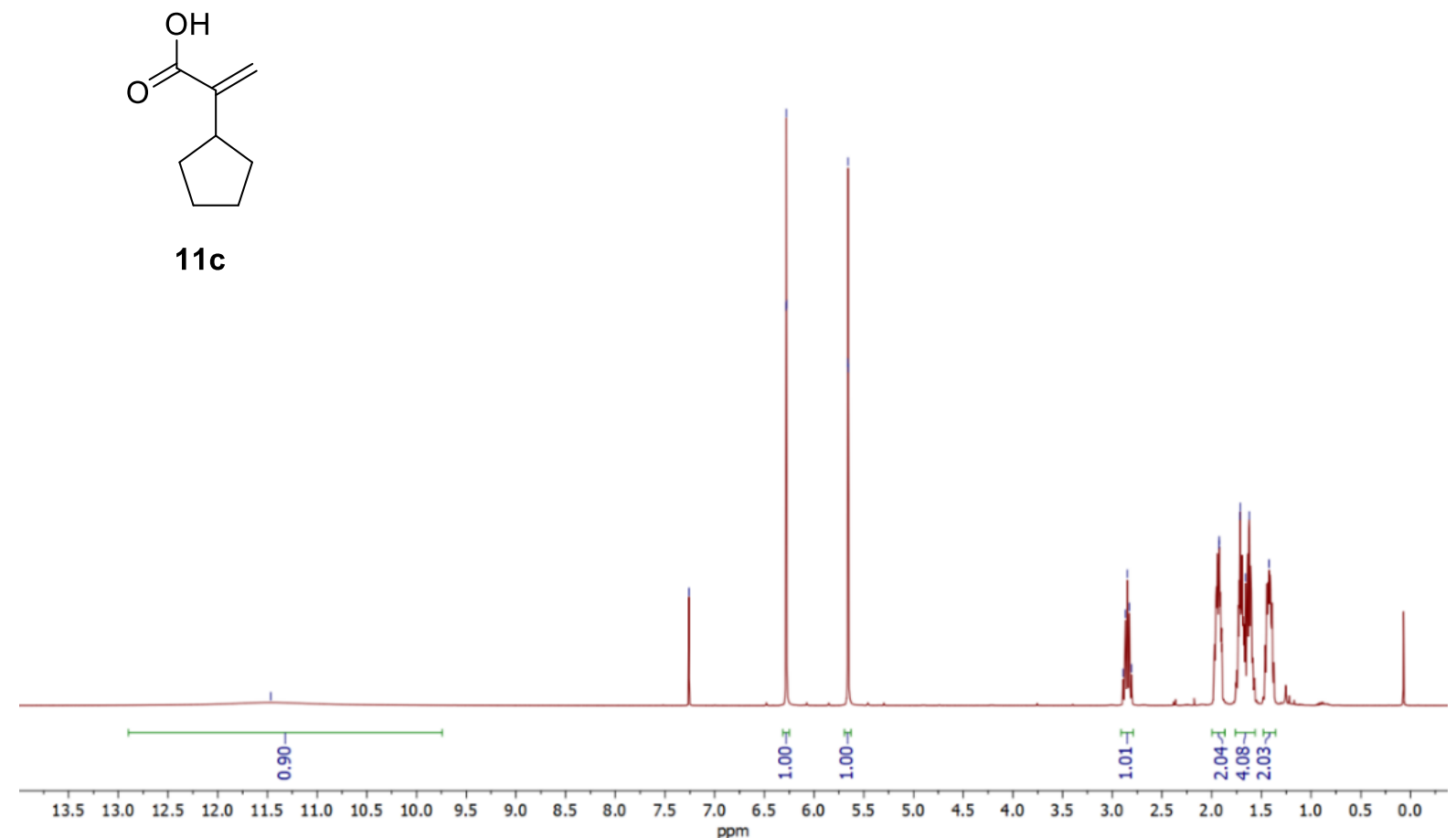

${ }^{13} \mathrm{C}\left\{{ }^{1} \mathrm{H}\right\} \operatorname{NMR}\left(\mathrm{CDCl}_{3}, 100.6 \mathrm{MHz}\right)$

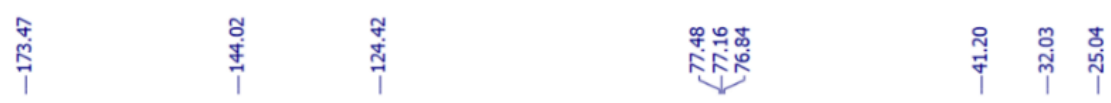<smiles>C=C(C(=O)O)C1CCCC1</smiles>

11c

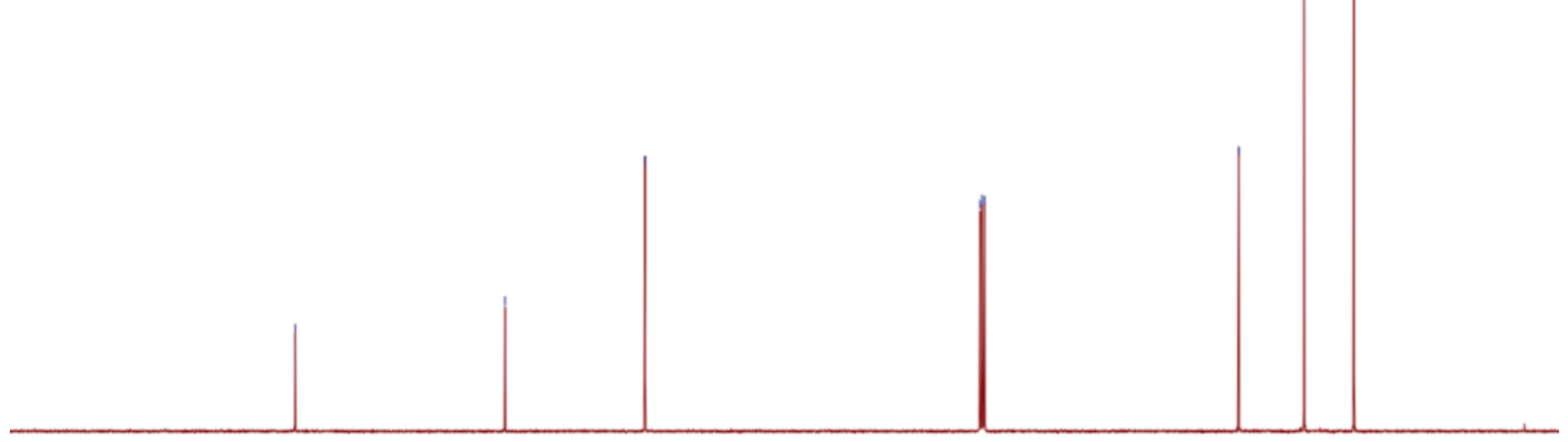

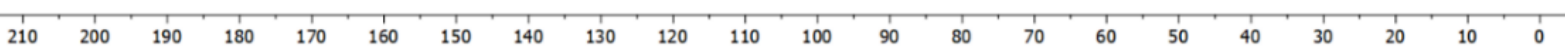




\section{b. a-Alkyl acrylamides}

${ }^{1} \mathrm{H} \mathrm{NMR}\left(\mathrm{CDCl}_{3}, 400 \mathrm{MHz}\right)$

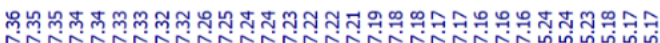

1.<smiles>C=C(C)C(=O)N(c1ccccc1)c1ccccc1</smiles>

$1 b$

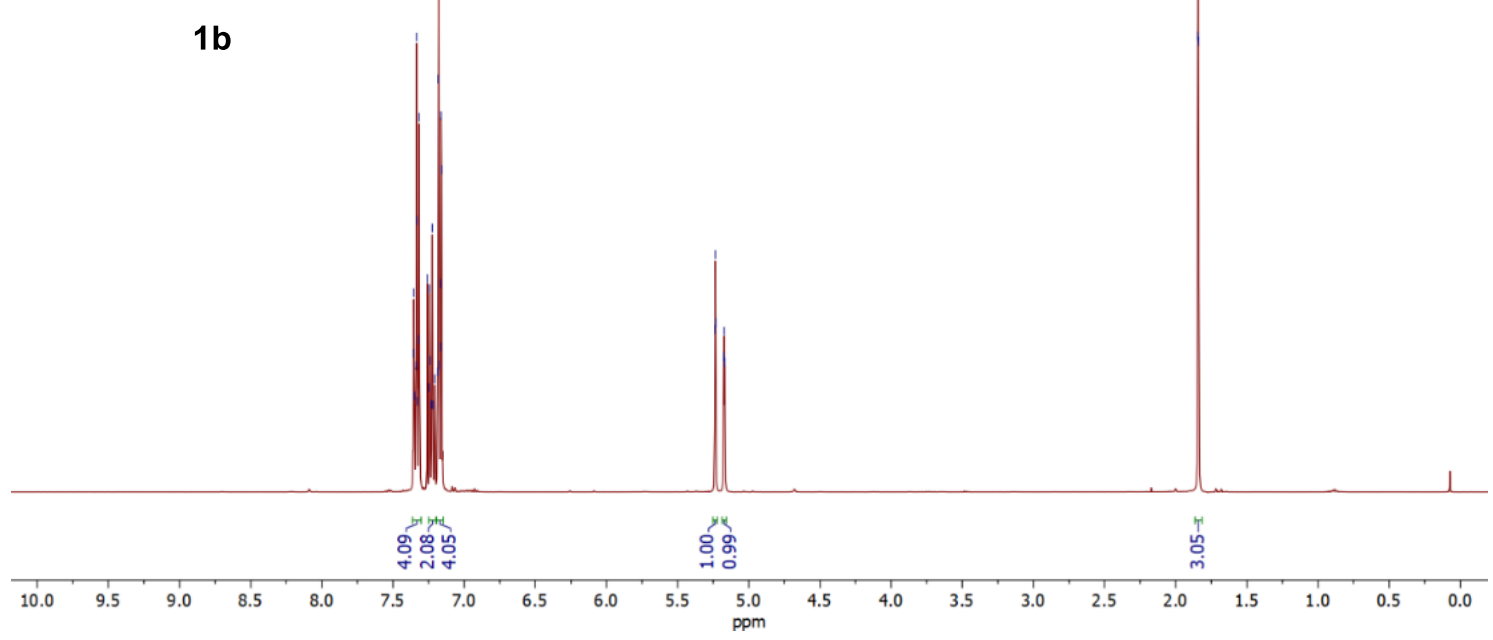

${ }^{13} \mathrm{C}\left\{{ }^{1} \mathrm{H}\right\} \operatorname{NMR}\left(\mathrm{CDCl}_{3}, 100.6 \mathrm{MHz}\right)$

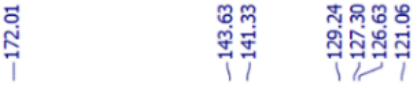

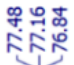

กั่<smiles>C=C(C)C(=O)N(c1ccccc1)c1ccccc1</smiles>

$1 b$

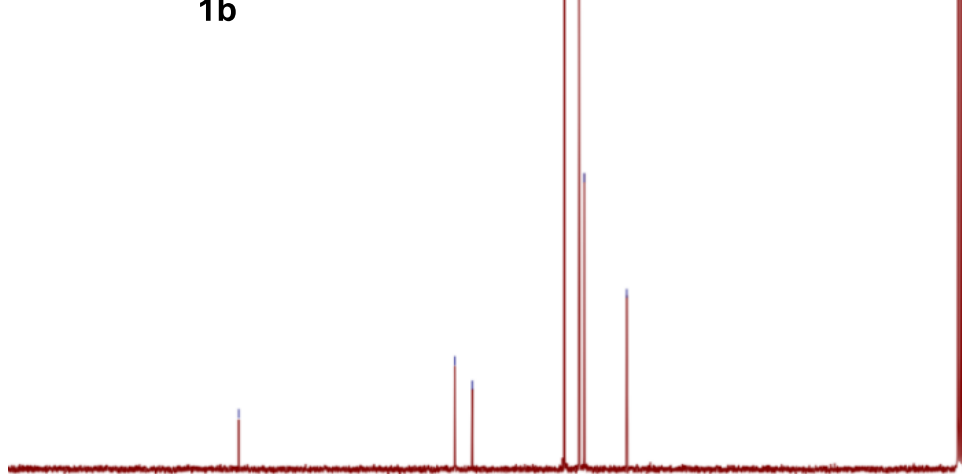

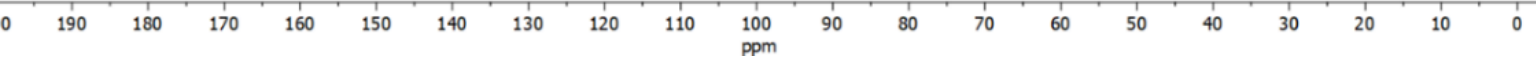


${ }^{1} \mathrm{H} \mathrm{NMR}\left(\mathrm{CDCl}_{3}, 400 \mathrm{MHz}\right)$

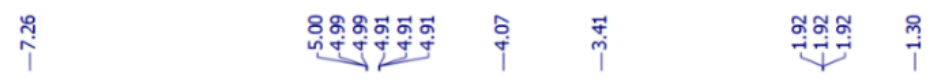<smiles>C=C(C)C(=O)N(C(C)C)C(C)C</smiles>

1c

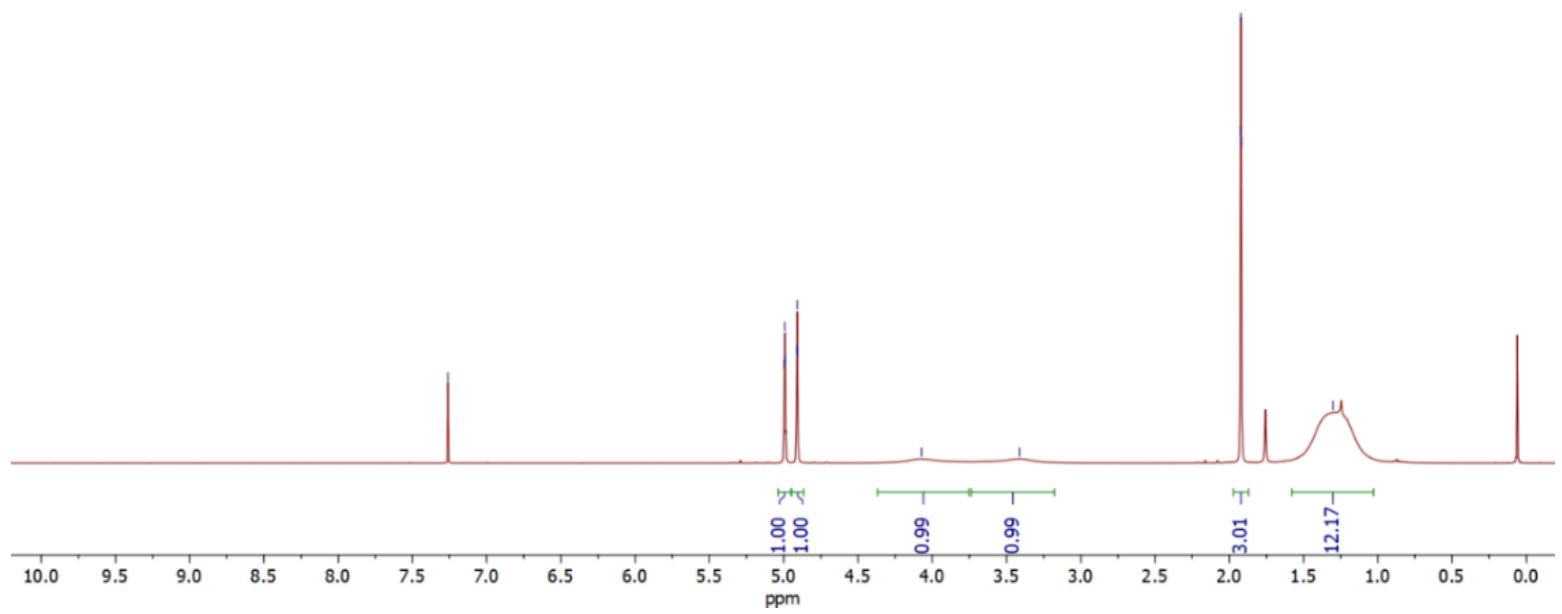

${ }^{13} \mathrm{C}\left\{{ }^{1} \mathrm{H}\right\}$ NMR $\left(\mathrm{CDCl}_{3}, 100.6 \mathrm{MHz}\right)$

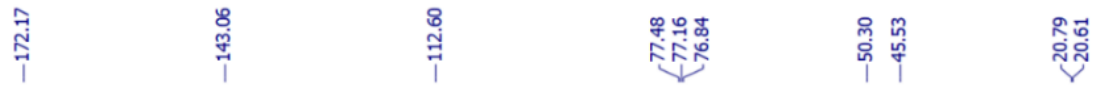

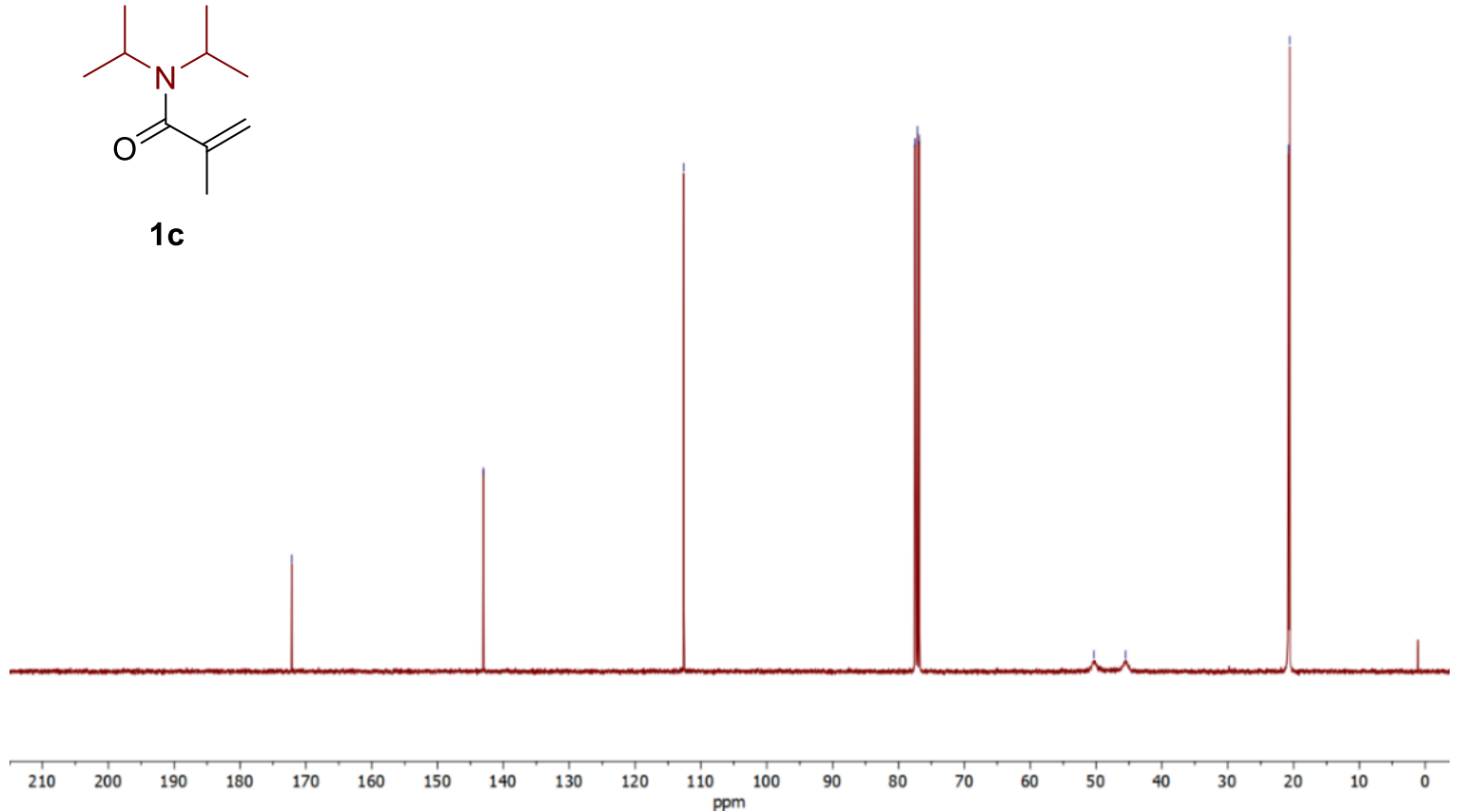


${ }^{1} \mathrm{H} \mathrm{NMR}\left(\mathrm{CDCl}_{3}, 400 \mathrm{MHz}\right)$
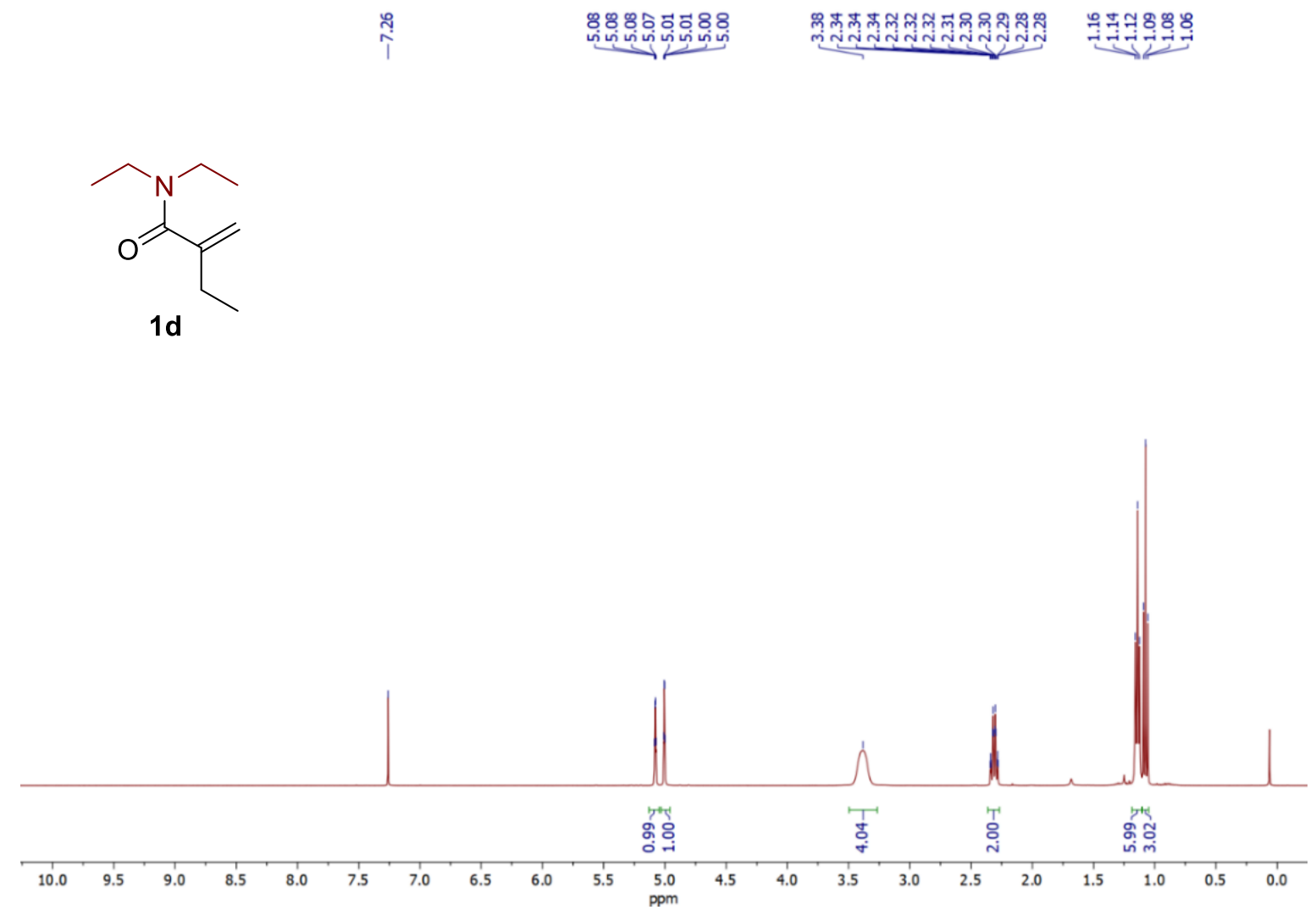

${ }^{13} \mathrm{C}\left\{{ }^{1} \mathrm{H}\right\}$ NMR $\left(\mathrm{CDCl}_{3}, 100.6 \mathrm{MHz}\right)$

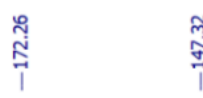

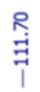

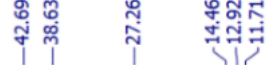<smiles>C=C(CC)C(=O)N(CC)CC</smiles>

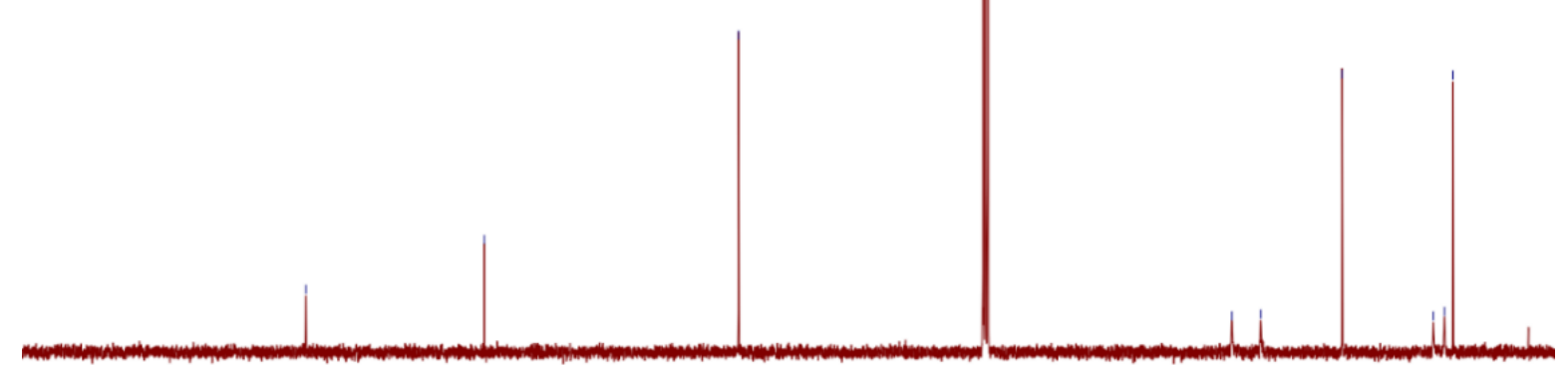

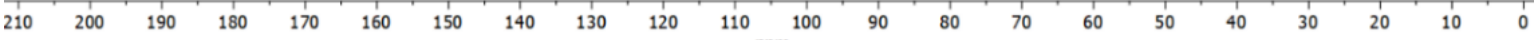


${ }^{1} \mathrm{H} \mathrm{NMR}\left(\mathrm{CDCl}_{3}, 400 \mathrm{MHz}\right)$
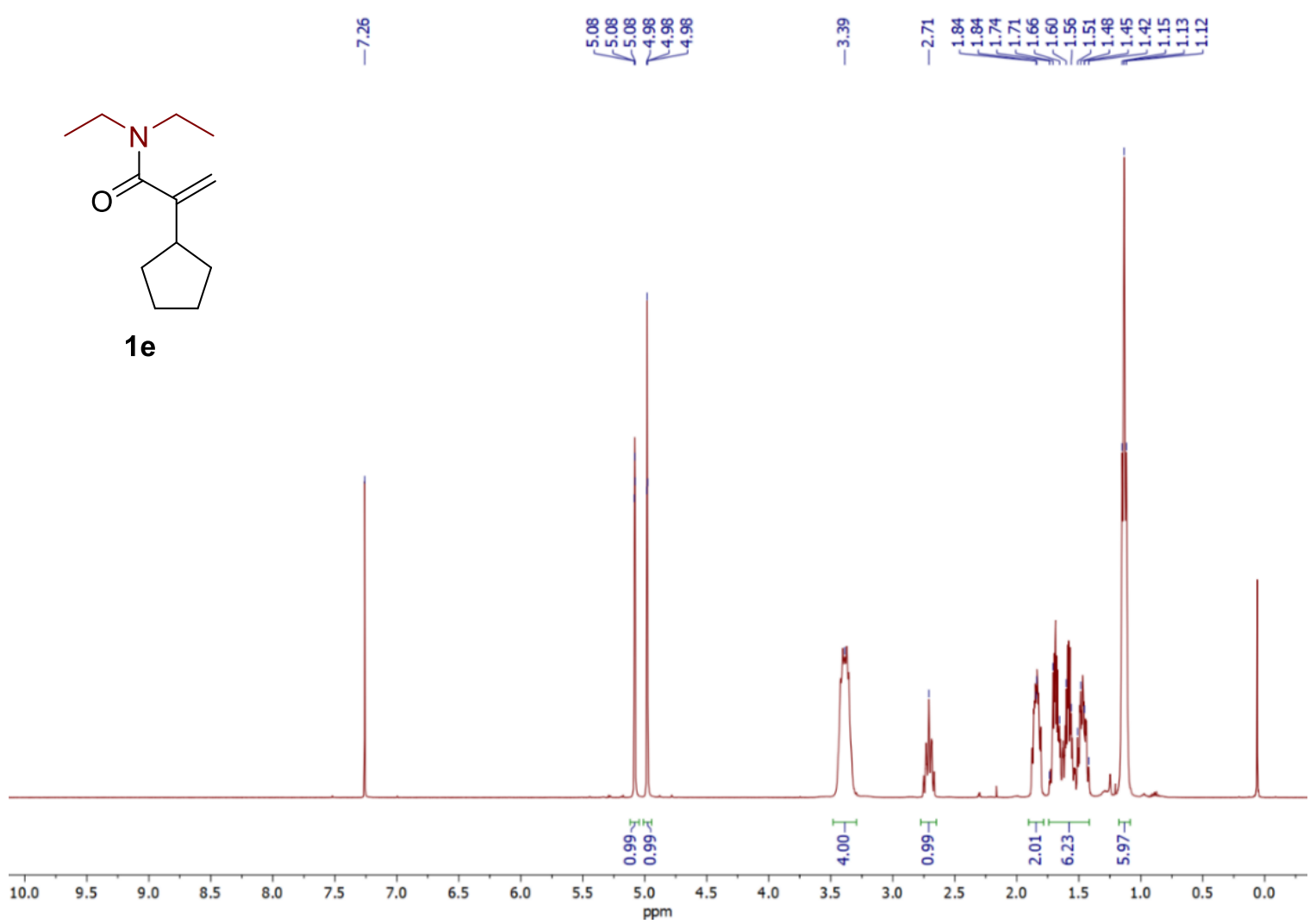

${ }^{13} \mathrm{C}\left\{{ }^{1} \mathrm{H}\right\} \operatorname{NMR}\left(\mathrm{CDCl}_{3}, 100.6 \mathrm{MHz}\right)$

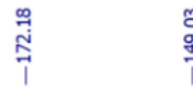

:

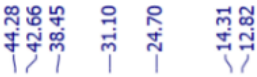

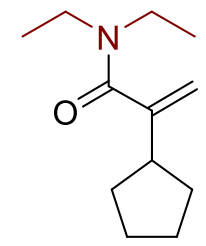

$1 e$
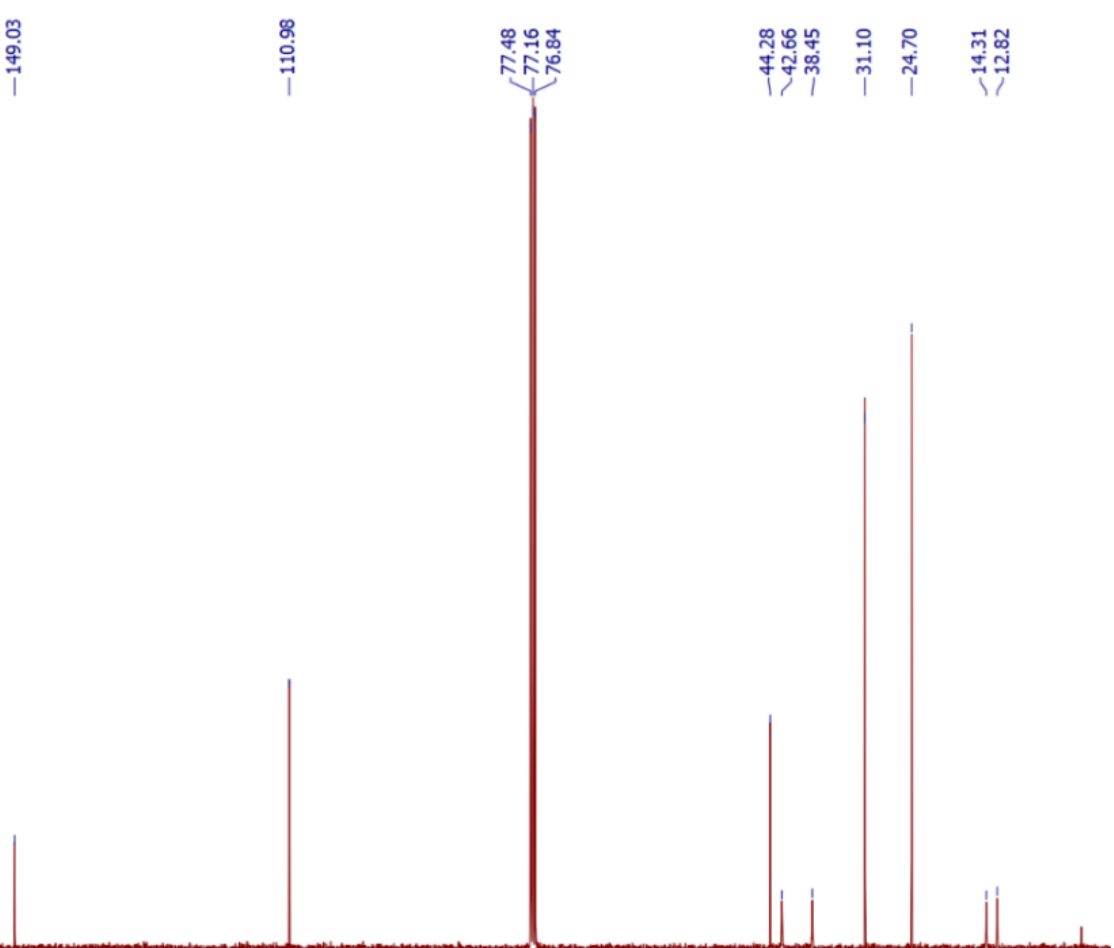

\begin{tabular}{lllllllllllllllllllllllllllllllllll}
\hline & 1 & 200 & 190 & 180 & 170 & 160 & 150 & 140 & 130 & 120 & 110 & 100 & 90 & 80 & 70 & 60 & 50 & 40 & 30 & 20 & 10 & 0
\end{tabular} 
${ }^{1} \mathrm{H} \mathrm{NMR}\left(\mathrm{CDCl}_{3}, 400 \mathrm{MHz}\right)$

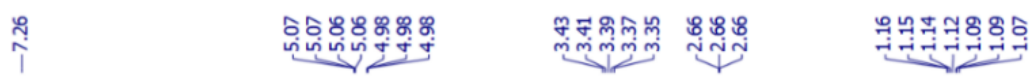<smiles>C=C(C(=O)N(CC)CC)C(C)C</smiles>

$1 f$

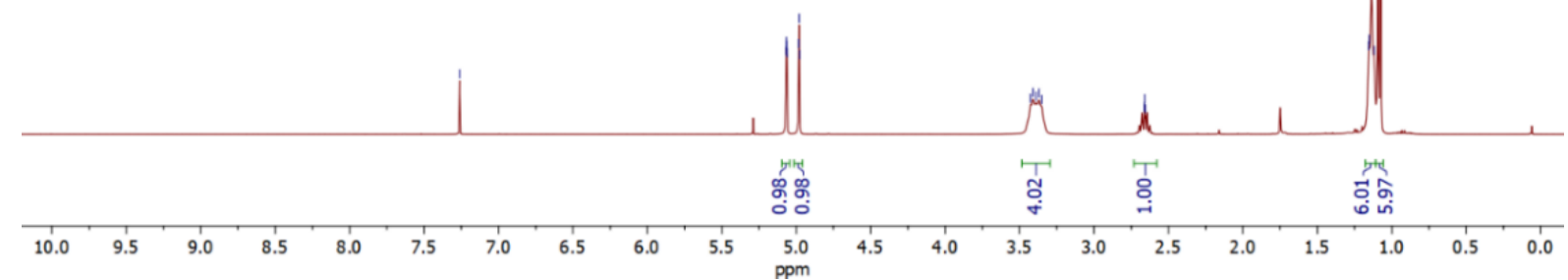

${ }^{13} \mathrm{C}\left\{{ }^{1} \mathrm{H}\right\}$ NMR $\left(\mathrm{CDCl}_{3}, 100.6 \mathrm{MHz}\right)$

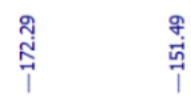

?.

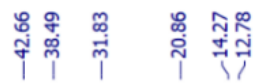<smiles>C=C(C(=O)N(CC)CC)C(C)C</smiles>

$1 f$

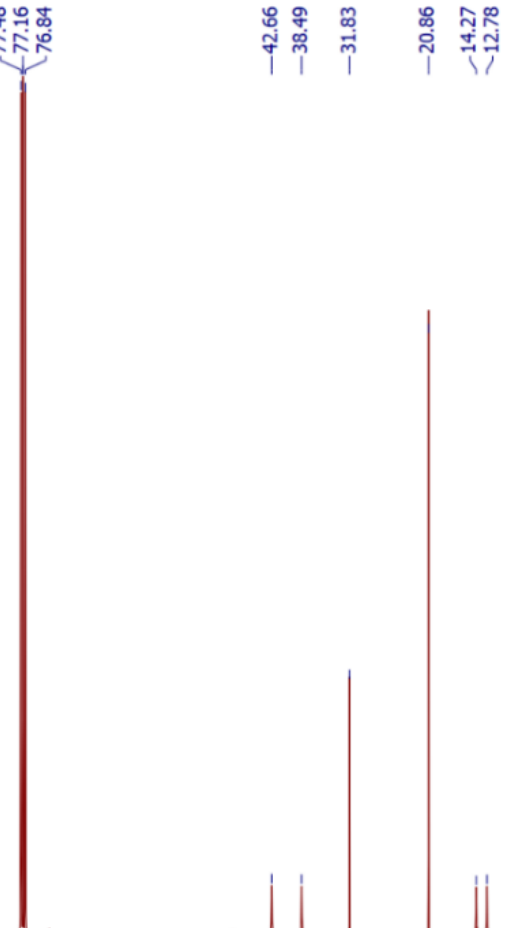

$\begin{array}{llllllllllll}210 & 200 & 190 & 180 & 170 & 160 & 150 & 140 & 130 & 120 & 110 & 100 \\ \mathrm{ppm}\end{array}$ 
${ }^{1} \mathrm{H} \mathrm{NMR}\left(\mathrm{CDCl}_{3}, 400 \mathrm{MHz}\right)$<smiles>C=C(Cc1ccccc1)C(=O)N(CC)CC</smiles>

$1 \mathrm{~g}$

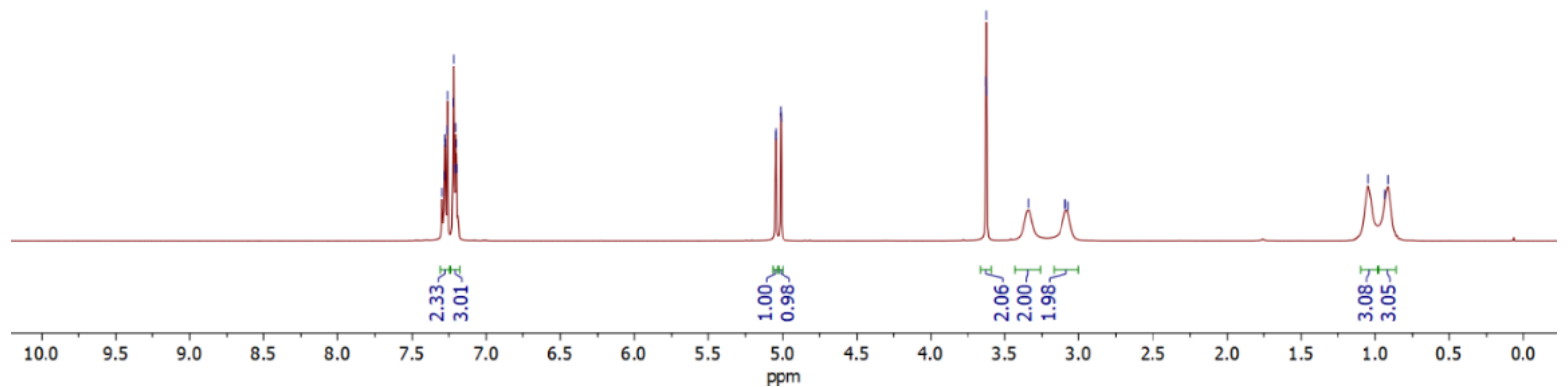

${ }^{13} \mathrm{C}\left\{{ }^{1} \mathrm{H}\right\} \operatorname{NMR}\left(\mathrm{CDCl}_{3}, 100.6 \mathrm{MHz}\right)$

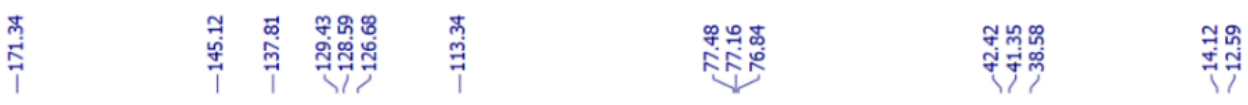<smiles>C=C(Cc1ccccc1)C(=O)N(CC)CC</smiles>

$1 \mathrm{~g}$

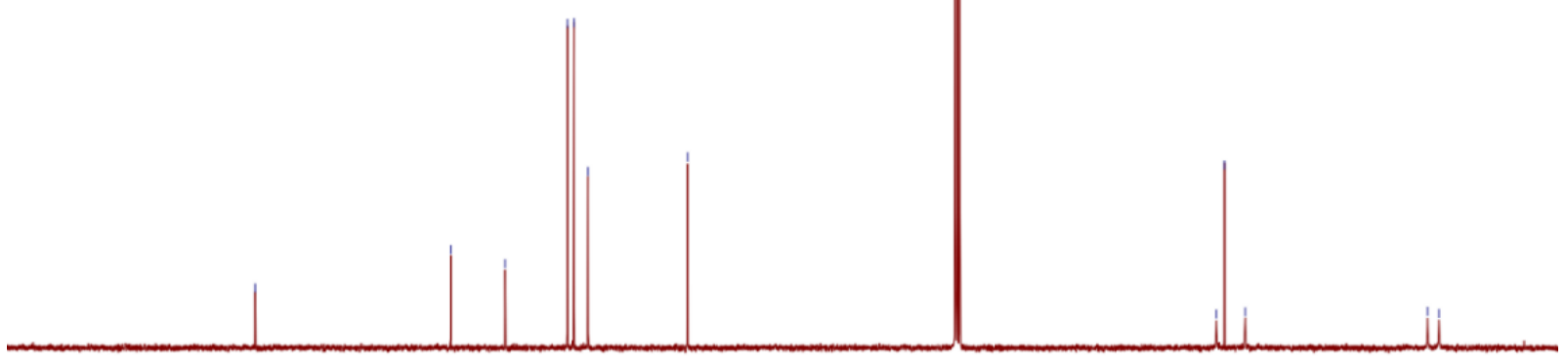

200
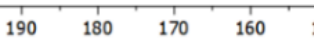

140

100
$\mathrm{ppm}$ 
${ }^{1} \mathrm{H} \mathrm{NMR}\left(\mathrm{CDCl}_{3}, 400 \mathrm{MHz}\right)$

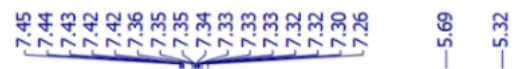<smiles>C=C(C(=O)N(CC)CC)c1ccccc1</smiles>

$1 \mathrm{~h}$

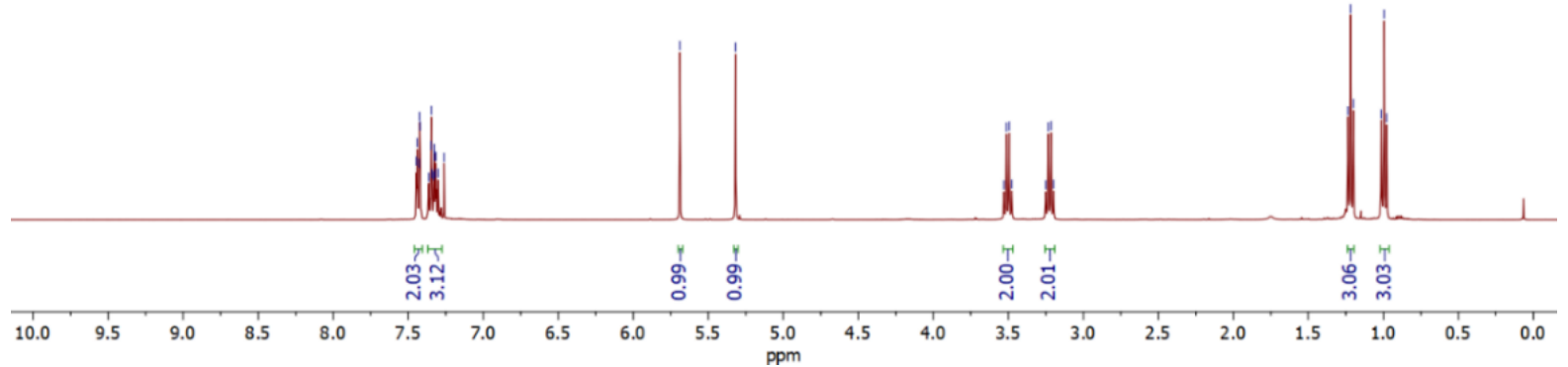

${ }^{13} \mathrm{C}\left\{{ }^{1} \mathrm{H}\right\} \operatorname{NMR}\left(\mathrm{CDCl}_{3}, 100.6 \mathrm{MHz}\right)$

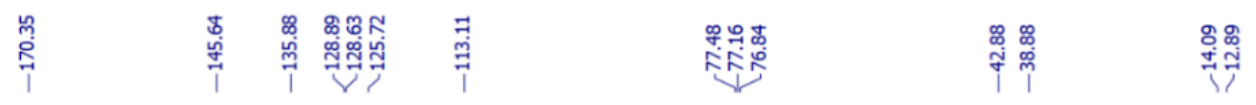<smiles>C=C(C(=O)N(CC)CC)c1ccccc1</smiles>

1h

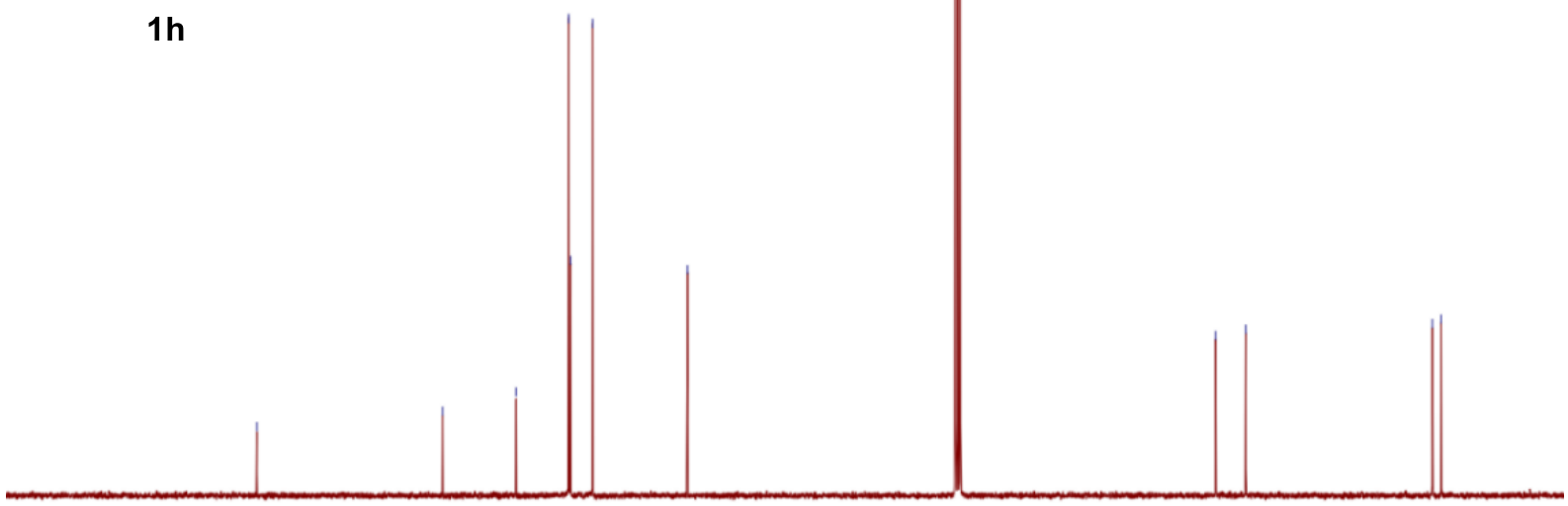

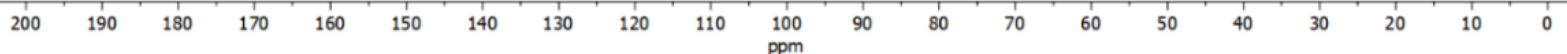



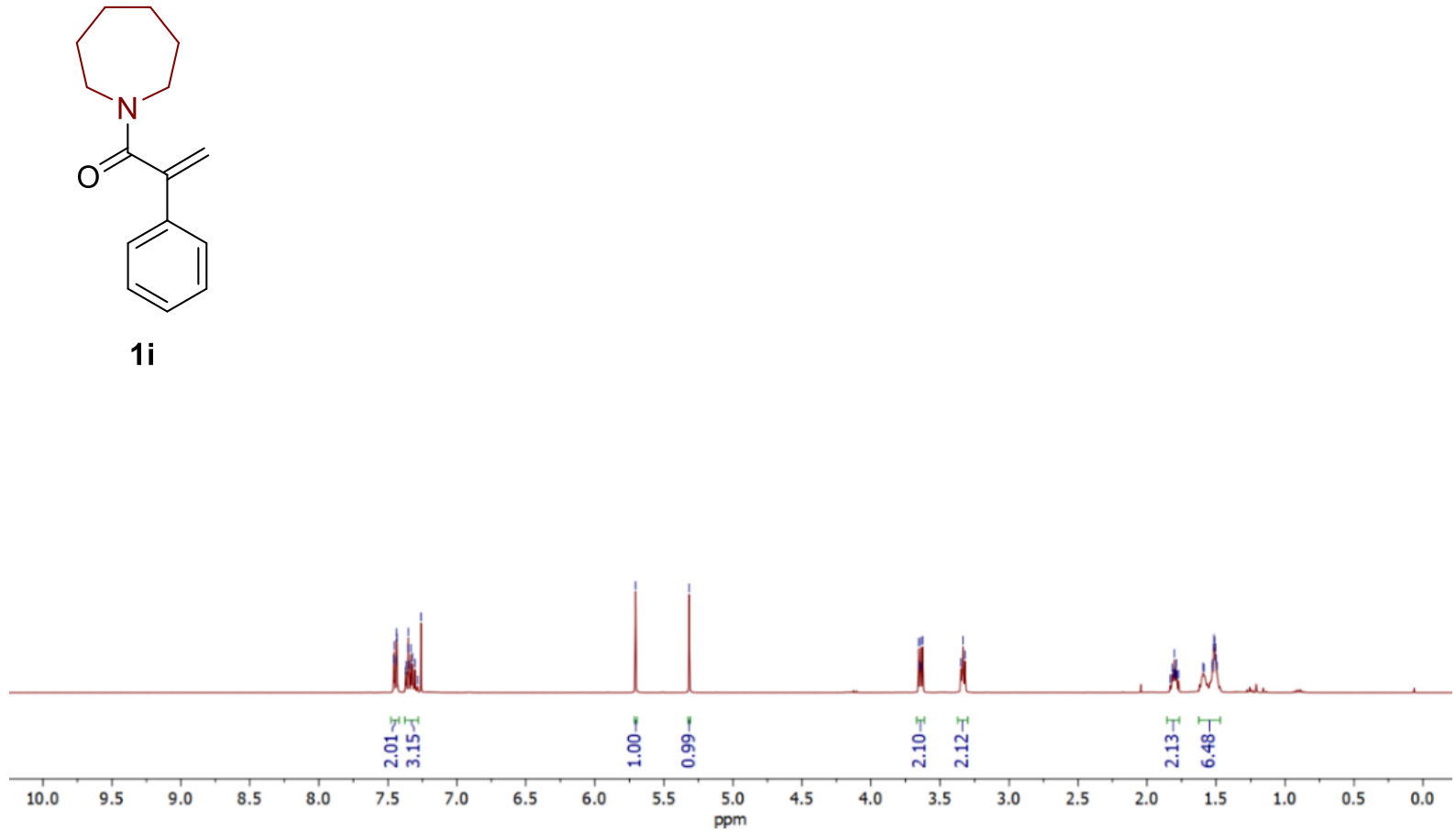

${ }^{13} \mathrm{C}\left\{{ }^{1} \mathrm{H}\right\} \operatorname{NMR}\left(\mathrm{CDCl}_{3}, 100.6 \mathrm{MHz}\right)$

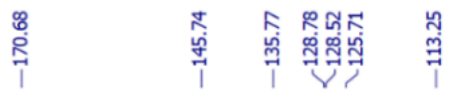<smiles>C=C(C(=O)N1CCCCCC1)c1ccccc1</smiles>

$1 \mathrm{i}$

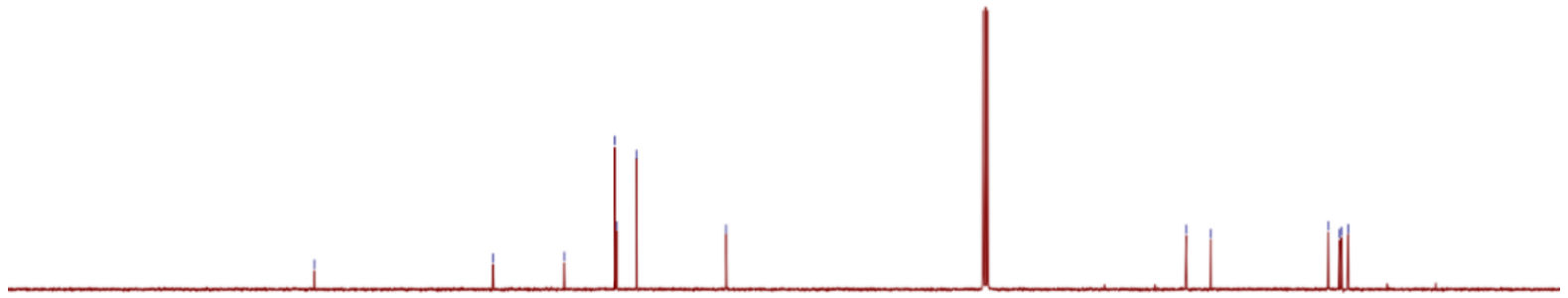

$\begin{array}{lllllllllllllllllllllllllllllllll}1 & 10 & 200 & 190 & 180 & 170 & 160 & 150 & 140 & 130 & 120 & 110 & 100 & 90 & 80 & 70 & 60 & 50 & 40 & 30 & 20 & 10 & 0\end{array}$ 


\section{c. Aldehydes}

${ }^{1} \mathrm{H} \mathrm{NMR}\left(\mathrm{CDCl}_{3}, 400 \mathrm{MHz}\right)$

$\stackrel{\substack{n \\ \text { o }}}{i}$

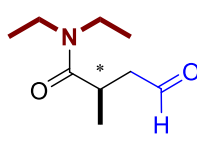

$2 a$

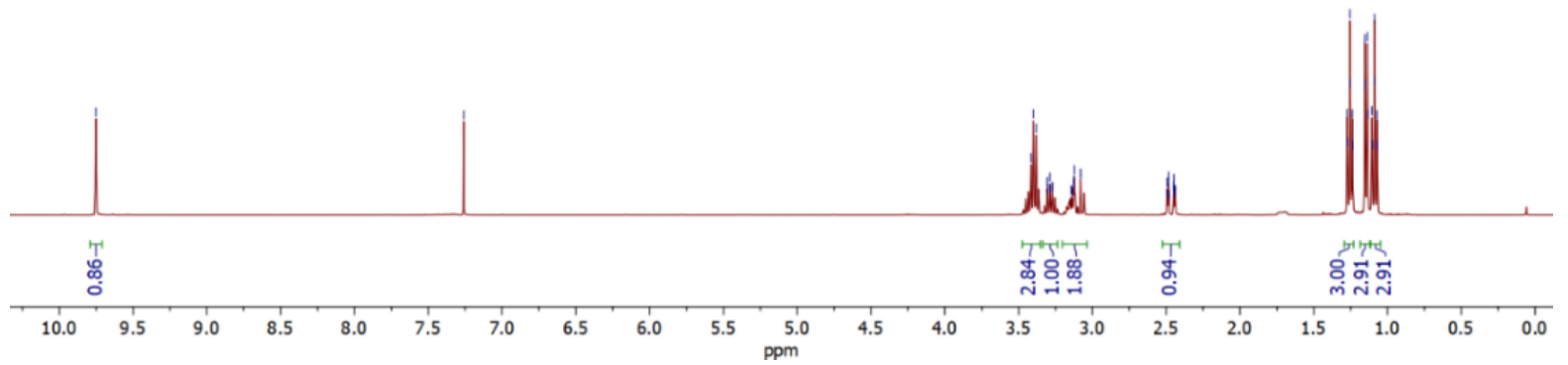

${ }^{13} \mathrm{C}\left\{{ }^{1} \mathrm{H}\right\}$ NMR $\left(\mathrm{CDCl}_{3}, 100.6 \mathrm{MHz}\right)$

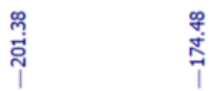

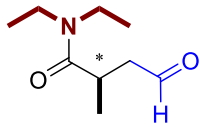

2a

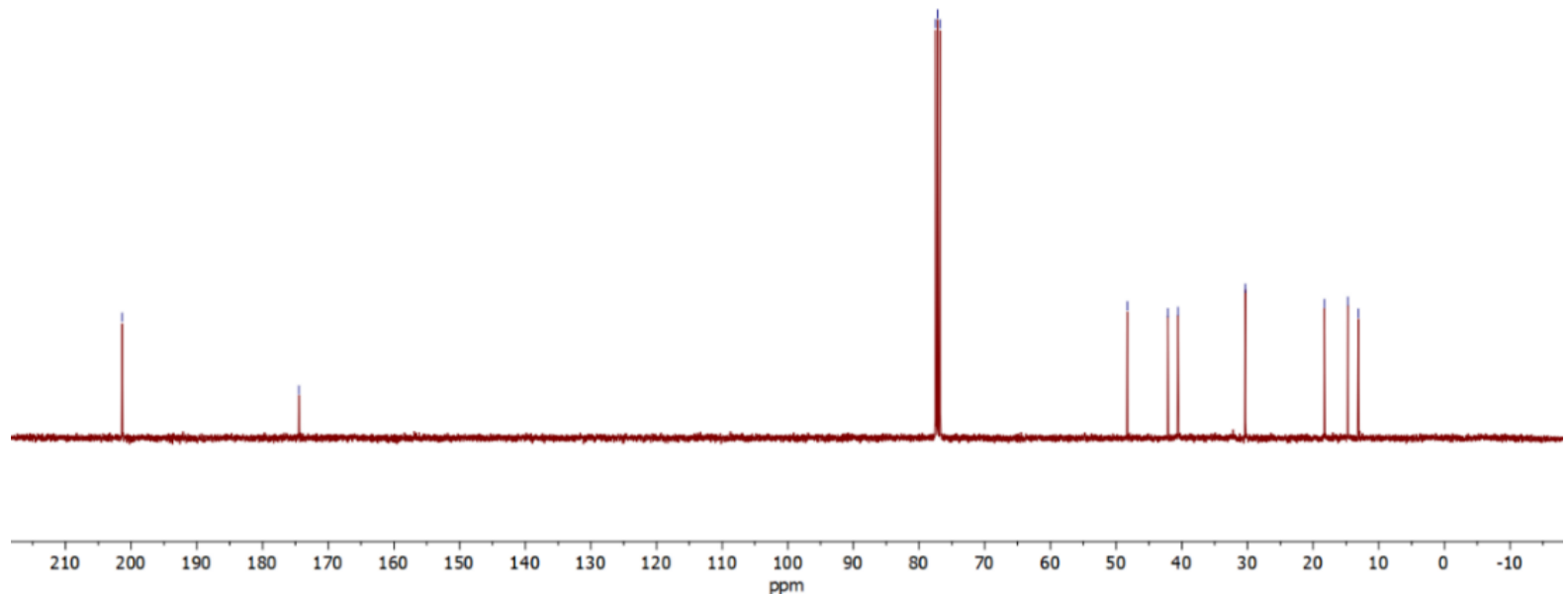


${ }^{1} \mathrm{H} \mathrm{NMR}\left(\mathrm{CDCl}_{3}, 400 \mathrm{MHz}\right)$

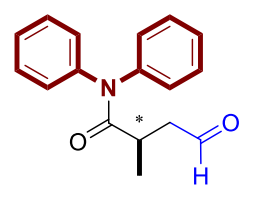

2b

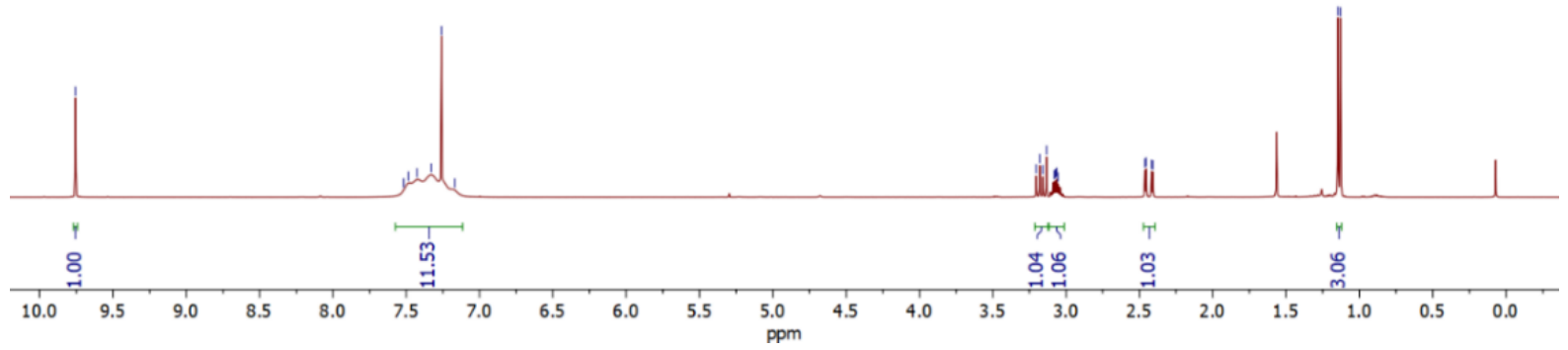

${ }^{13} \mathrm{C}\left\{{ }^{1} \mathrm{H}\right\} \operatorname{NMR}\left(\mathrm{CDCl}_{3}, 100.6 \mathrm{MHz}\right)$

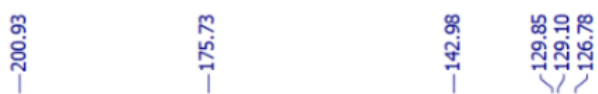<smiles>C1=CCC(Nc2ccccc2)CC1</smiles><smiles>CC(=CC=O)CC(C)=CC=O</smiles>

2b

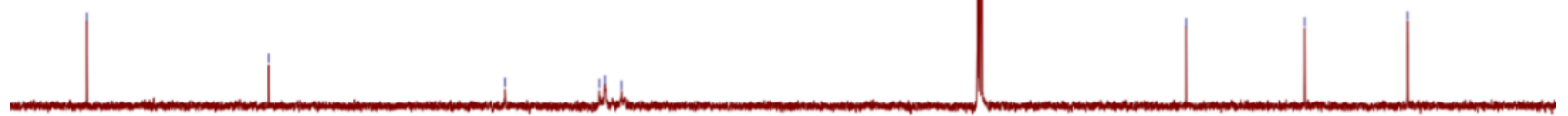

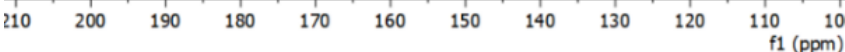


${ }^{1} \mathrm{H} \mathrm{NMR}\left(\mathrm{CDCl}_{3}, 400 \mathrm{MHz}\right)$

$\lambda$ 人

$\overbrace{2 c}^{*}$

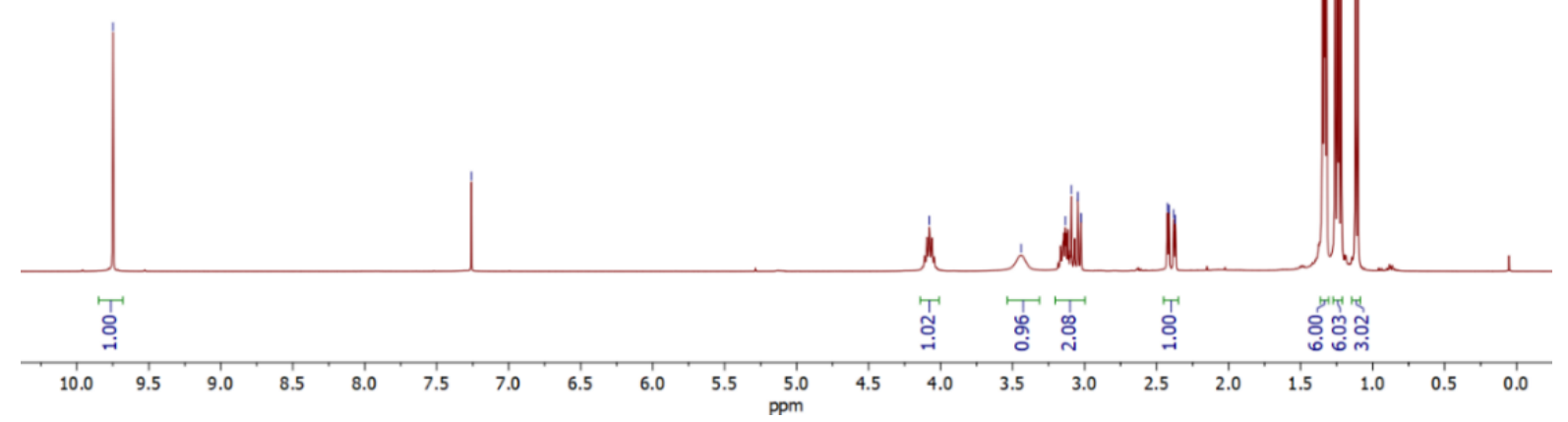

${ }^{13} \mathrm{C}\left\{{ }^{1} \mathrm{H}\right\} \mathrm{NMR}\left(\mathrm{CDCl}_{3}, 100.6 \mathrm{MHz}\right)$

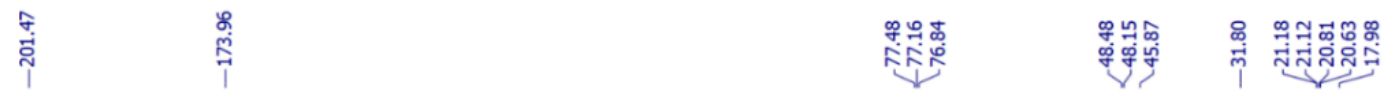

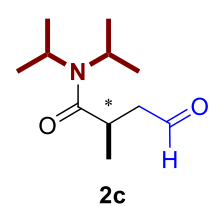

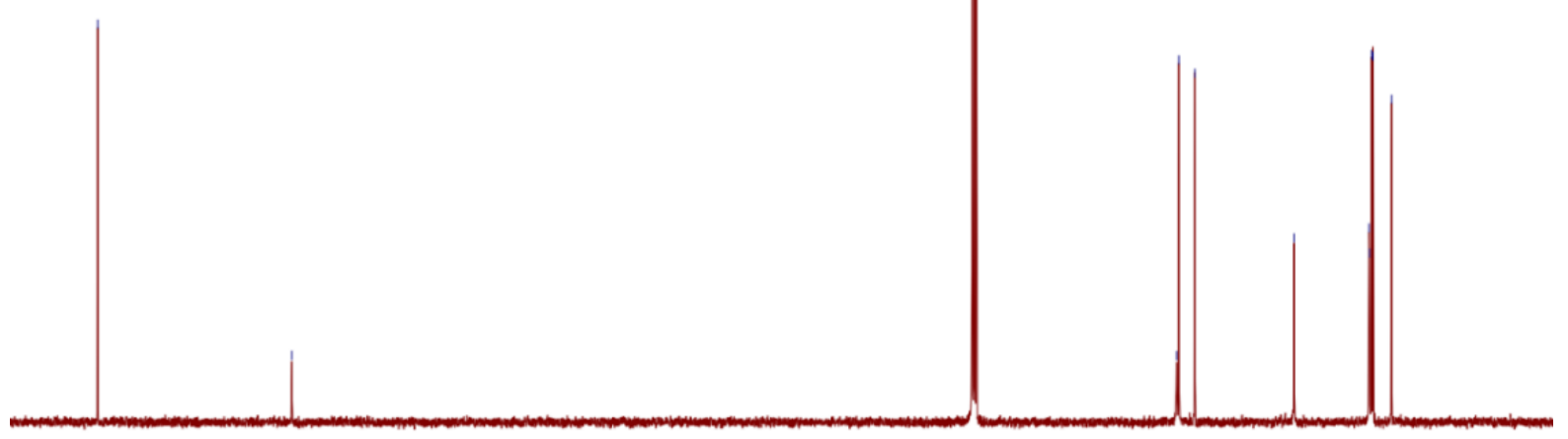

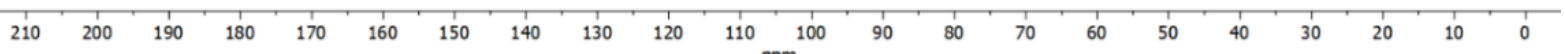


${ }^{1} \mathrm{H} \mathrm{NMR}\left(\mathrm{CDCl}_{3}, 400 \mathrm{MHz}\right)$

朵

i
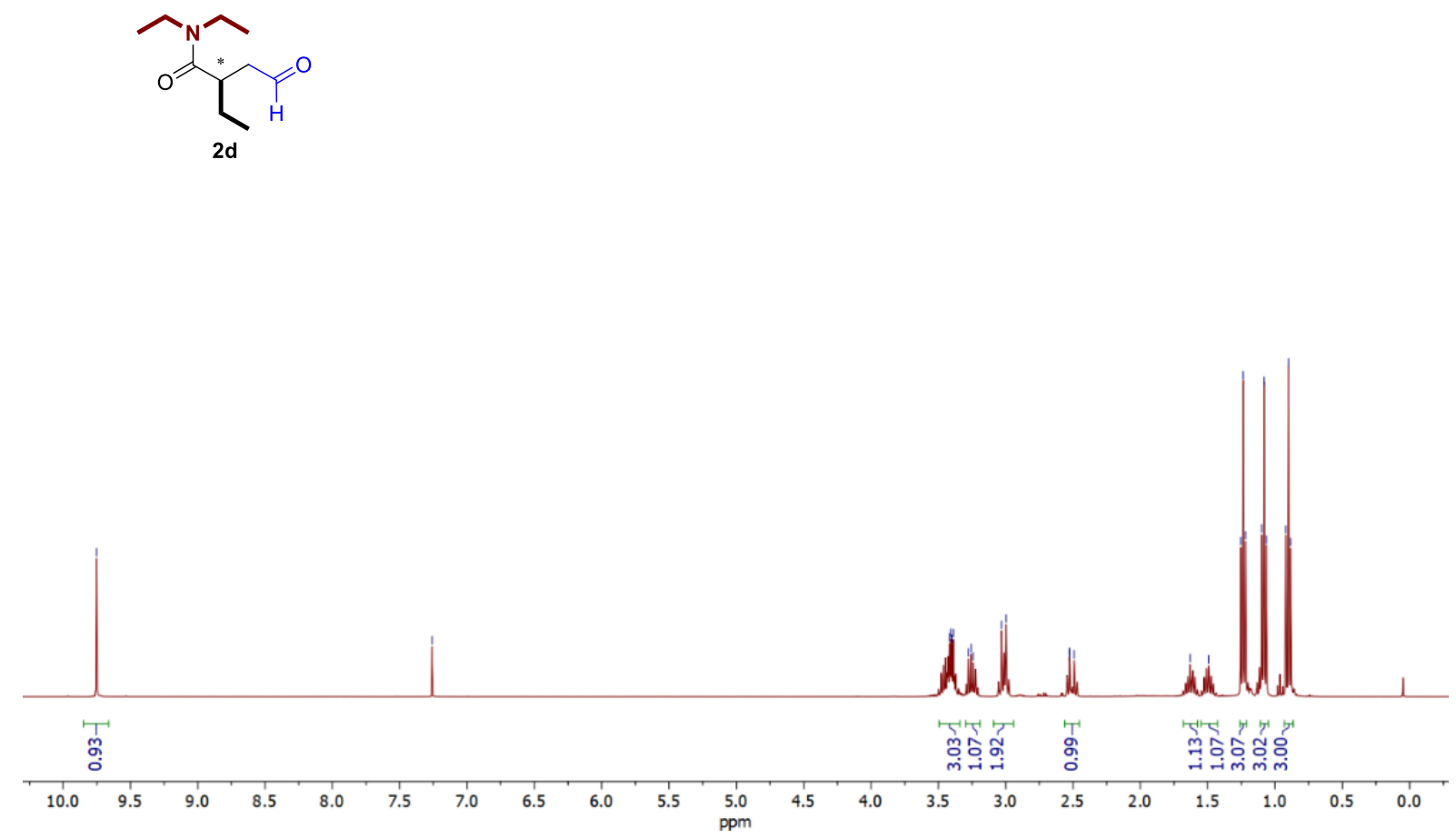

${ }^{13} \mathrm{C}\left\{{ }^{1} \mathrm{H}\right\} \mathrm{NMR}\left(\mathrm{CDCl}_{3}, 100.6 \mathrm{MHz}\right)$
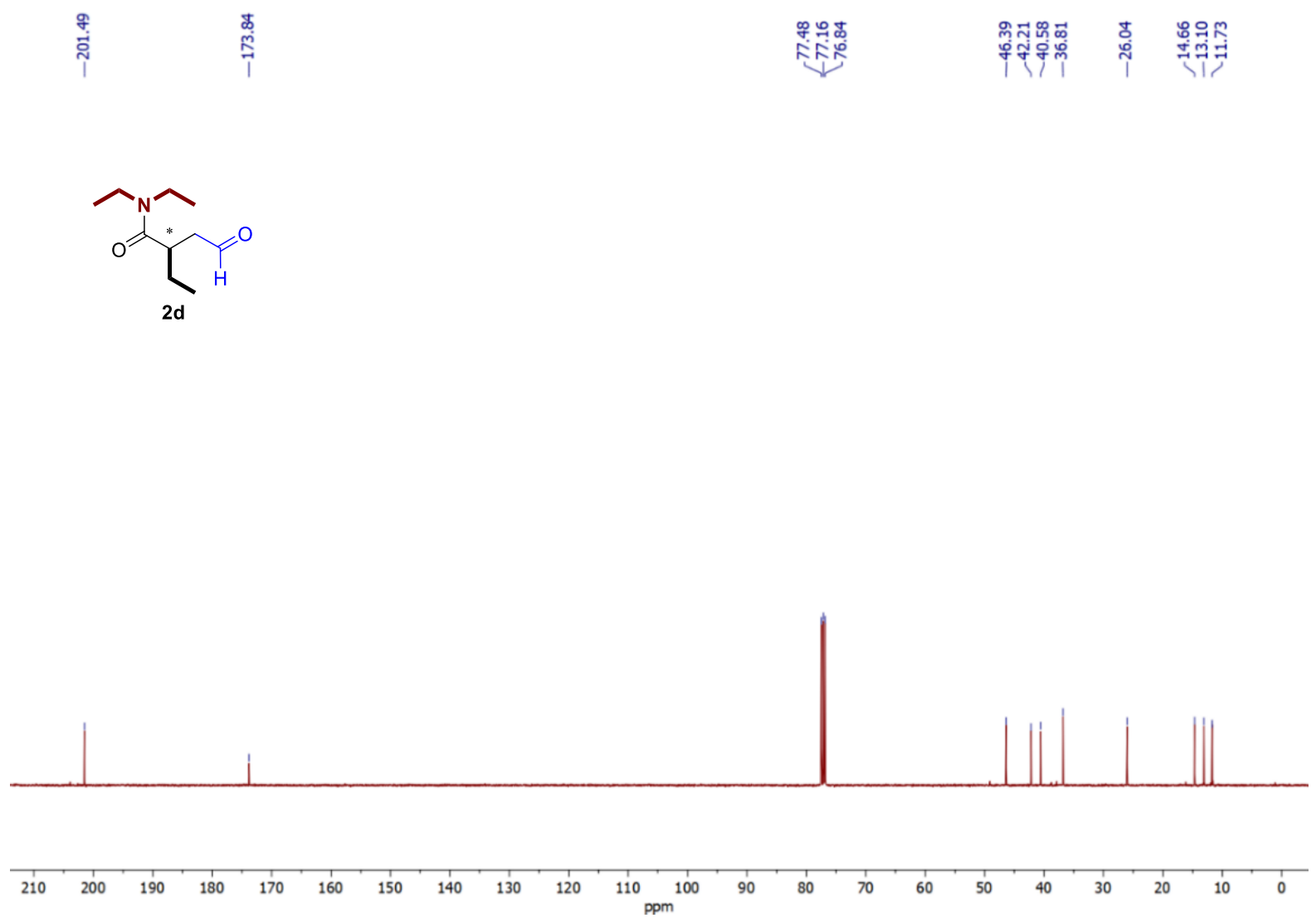
${ }^{1} \mathrm{H} \mathrm{NMR}\left(\mathrm{CDCl}_{3}, 400 \mathrm{MHz}\right)$
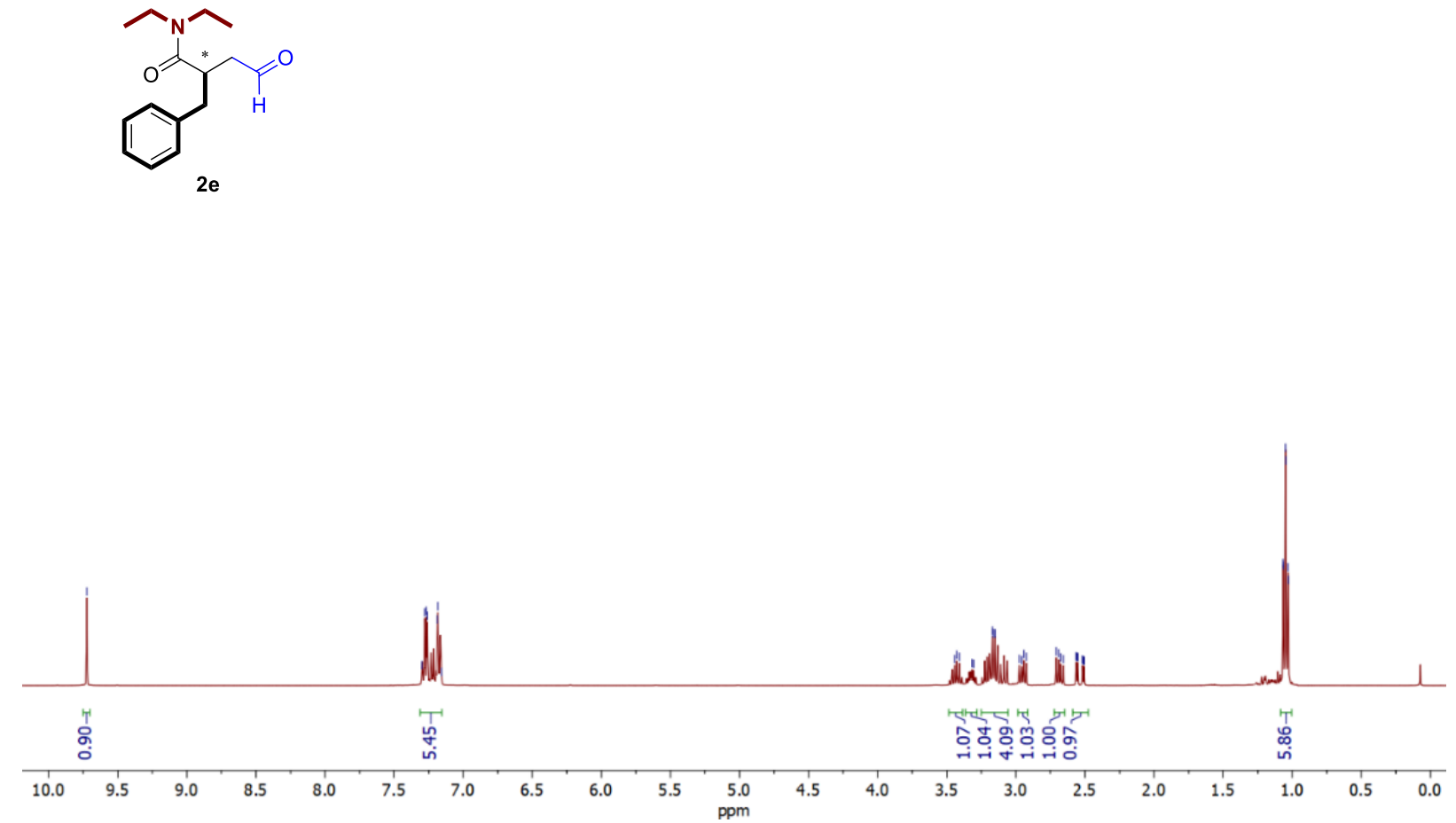

${ }^{13} \mathrm{C}\left\{{ }^{1} \mathrm{H}\right\} \operatorname{NMR}\left(\mathrm{CDCl}_{3}, 100.6 \mathrm{MHz}\right)$

\begin{tabular}{|c|c|c|c|}
\hline$\underset{\substack{\pi \\
\hdashline}}{\pi}$ & 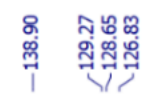 & 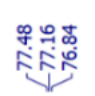 & 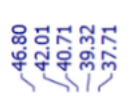 \\
\hline
\end{tabular}<smiles>CCN(CC)C(=O)CCC=O</smiles>

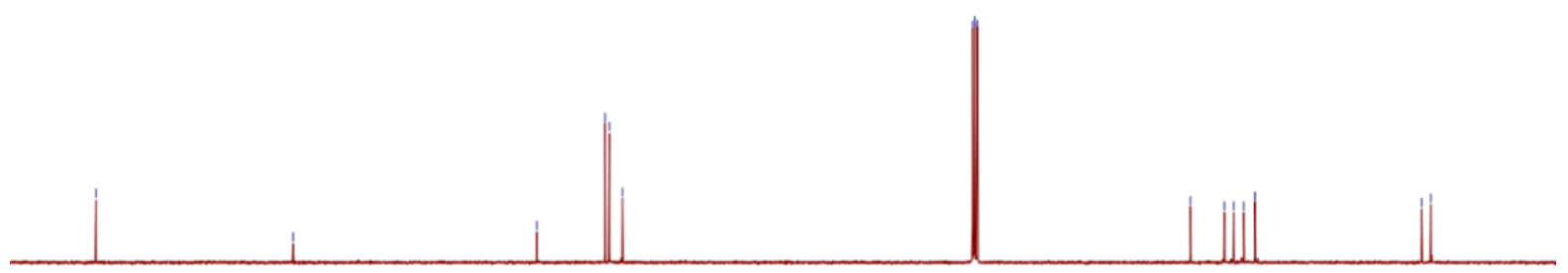

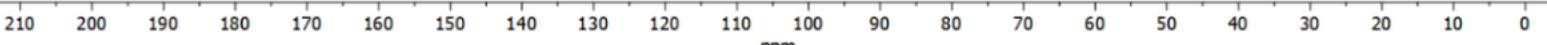


${ }^{1} \mathrm{H} \mathrm{NMR}\left(\mathrm{CDCl}_{3}, 400 \mathrm{MHz}\right)$

i

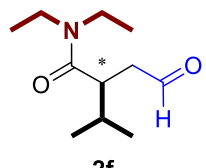

$2 f$

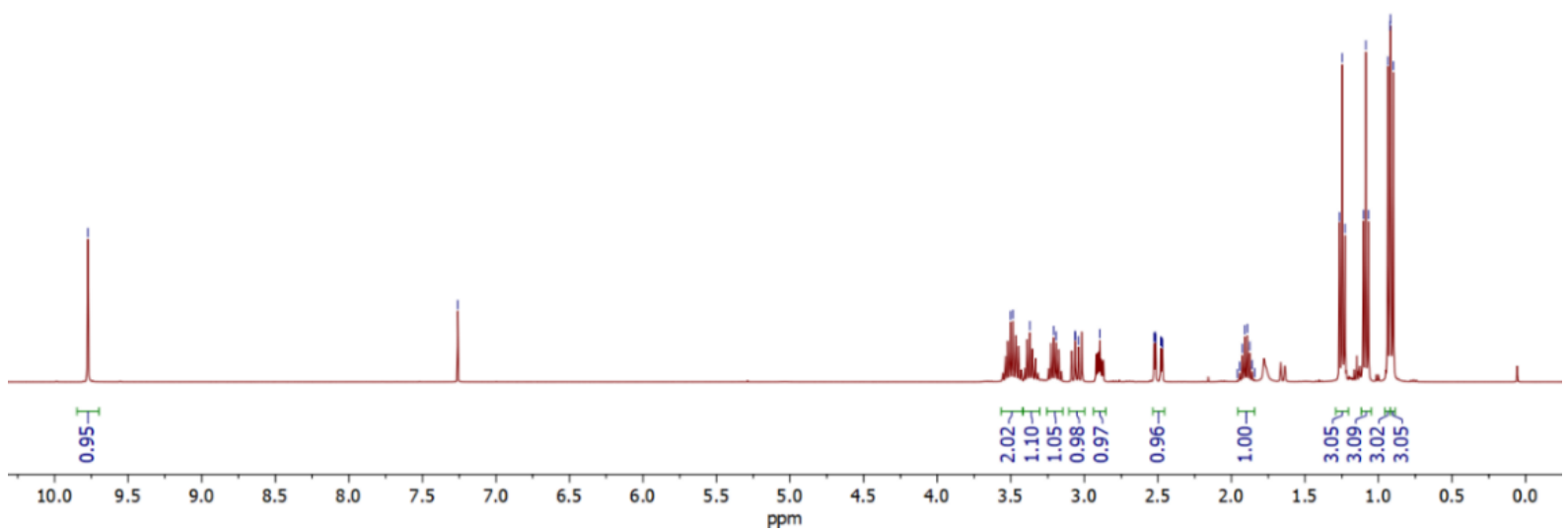

${ }^{13} \mathrm{C}\left\{{ }^{1} \mathrm{H}\right\} \operatorname{NMR}\left(\mathrm{CDCl}_{3}, 100.6 \mathrm{MHz}\right)$

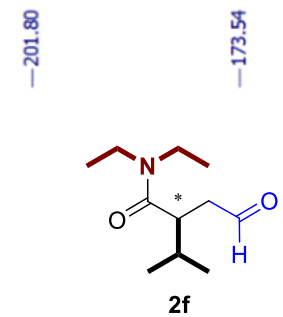

政

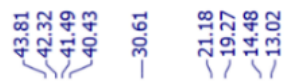

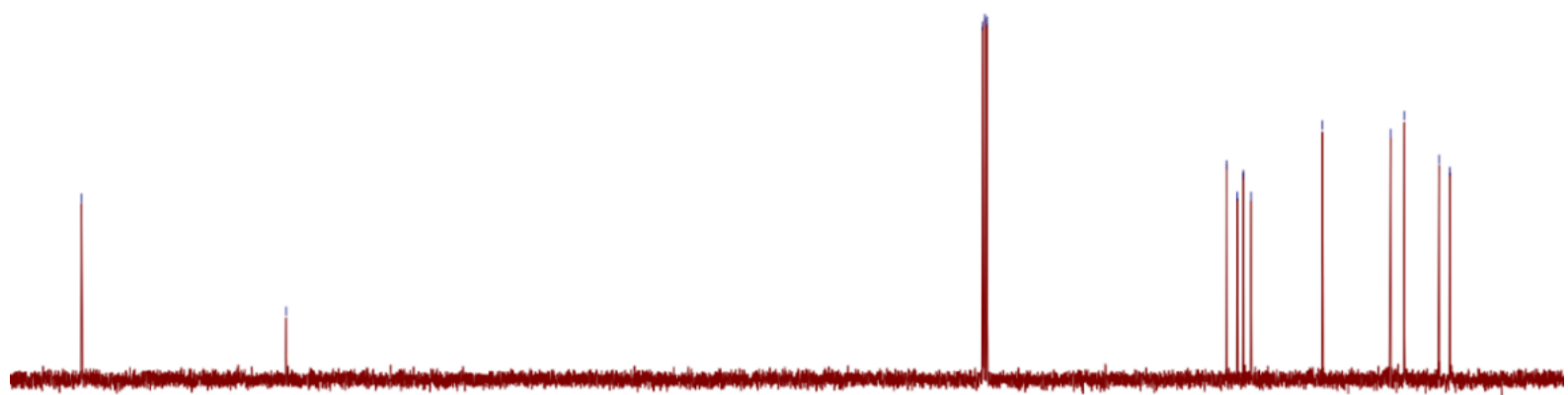

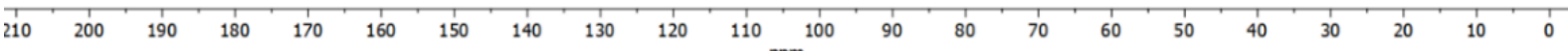


${ }^{1} \mathrm{H} \mathrm{NMR}\left(\mathrm{CDCl}_{3}, 400 \mathrm{MHz}\right)$

$\stackrel{\substack{n \\ \text { i }}}{i}$<smiles>CCN(CC)C(=O)C(CC=O)C1CCCC1</smiles>

$2 \mathrm{~g}$

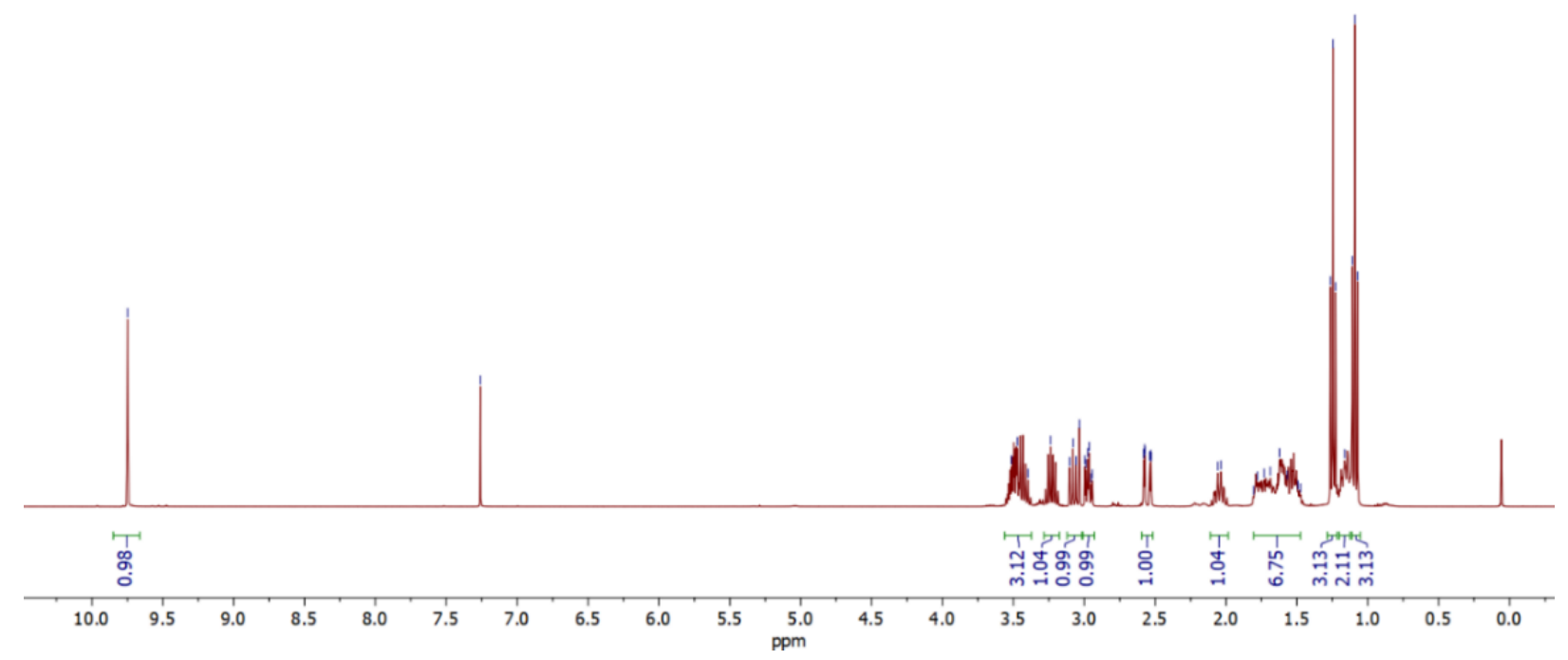

${ }^{13} \mathrm{C}\left\{{ }^{1} \mathrm{H}\right\} \mathrm{NMR}\left(\mathrm{CDCl}_{3}, 100.6 \mathrm{MHz}\right)$<smiles>[Te]=[Te]</smiles><smiles>CCN(CC)C(=O)C(CC=O)C1CCCCC1</smiles>

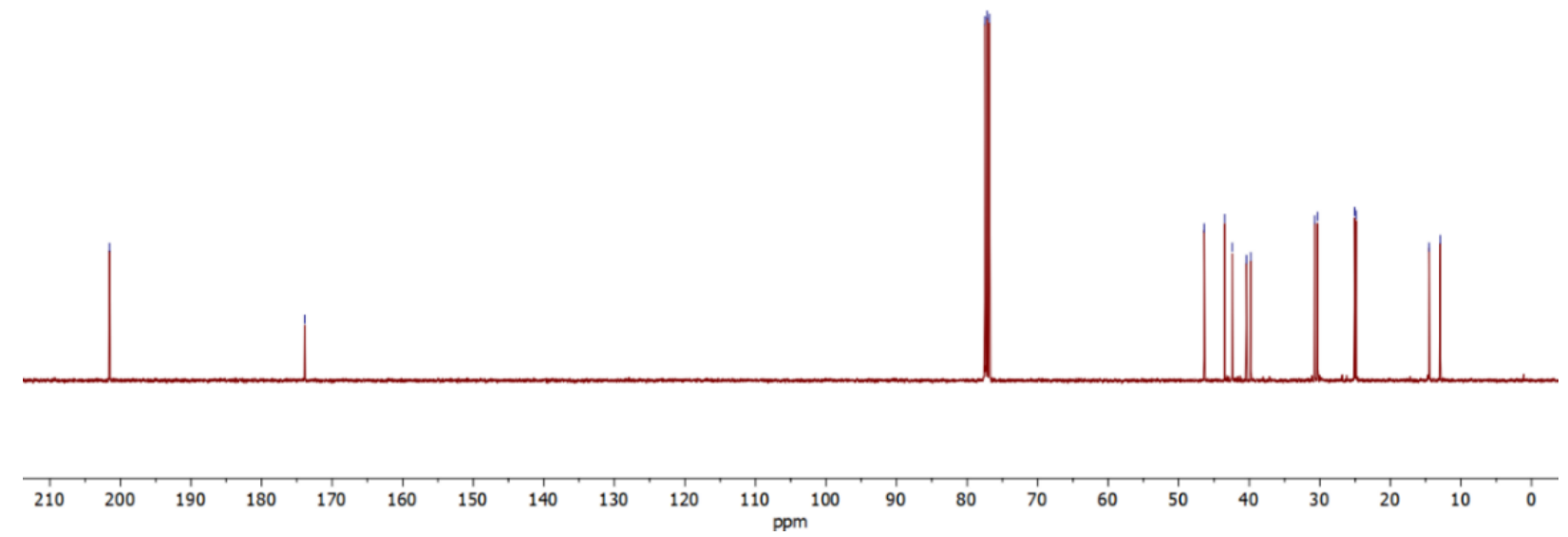


${ }^{1} \mathrm{H} \mathrm{NMR}\left(\mathrm{CDCl}_{3}, 400 \mathrm{MHz}\right)$

న<smiles>CCN(CC)C(=O)C(=CC=O)c1ccccc1</smiles>

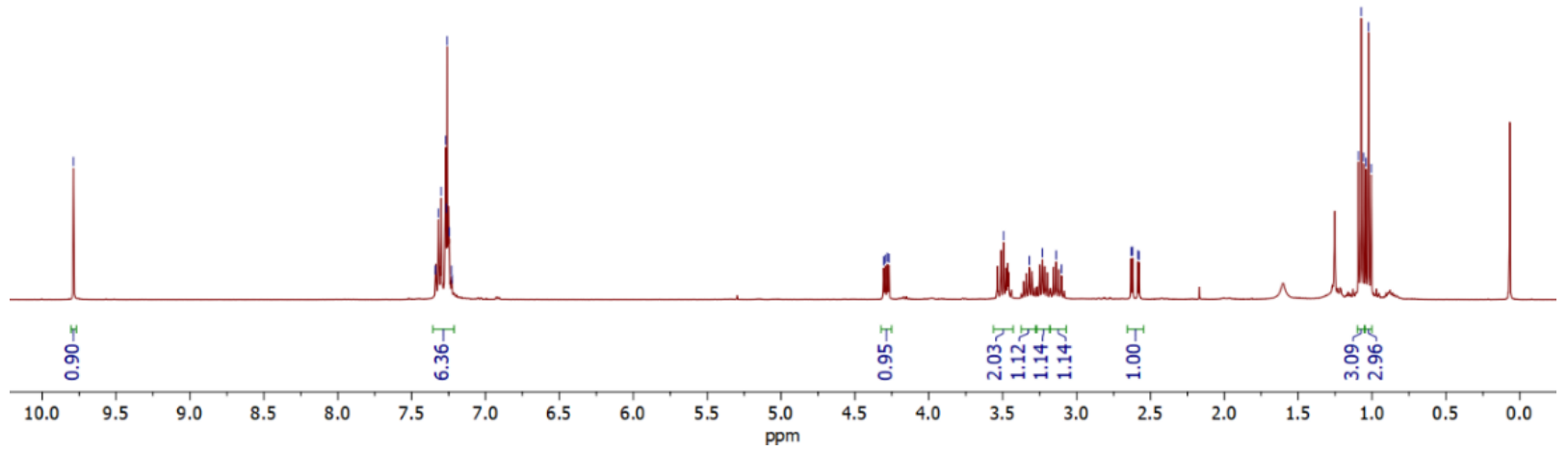

${ }^{13} \mathrm{C}\left\{{ }^{1} \mathrm{H}\right\} \operatorname{NMR}\left(\mathrm{CDCl}_{3}, 100.6 \mathrm{MHz}\right)$

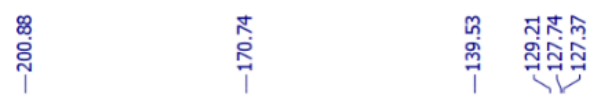<smiles>CCN(CC)C(=O)C(CC=O)c1ccccc1</smiles>

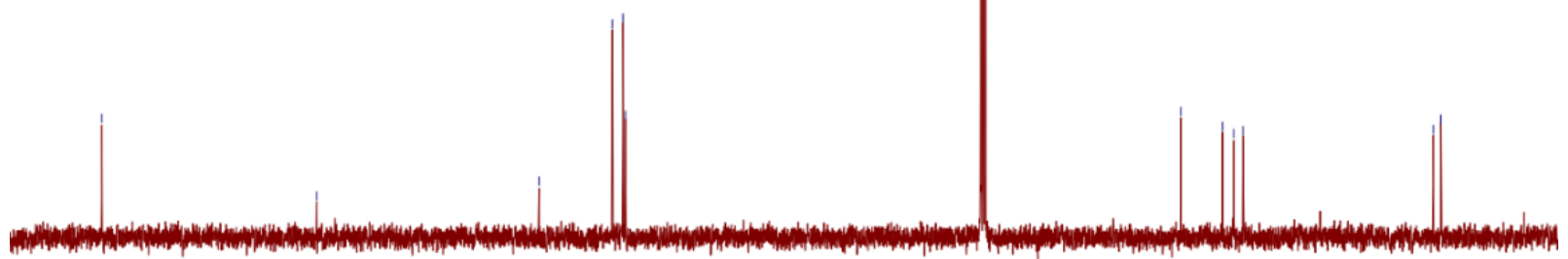

210

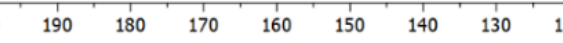


d. Alcohols

${ }^{1} \mathrm{H} \mathrm{NMR}\left(\mathrm{CDCl}_{3}, 400 \mathrm{MHz}\right)$

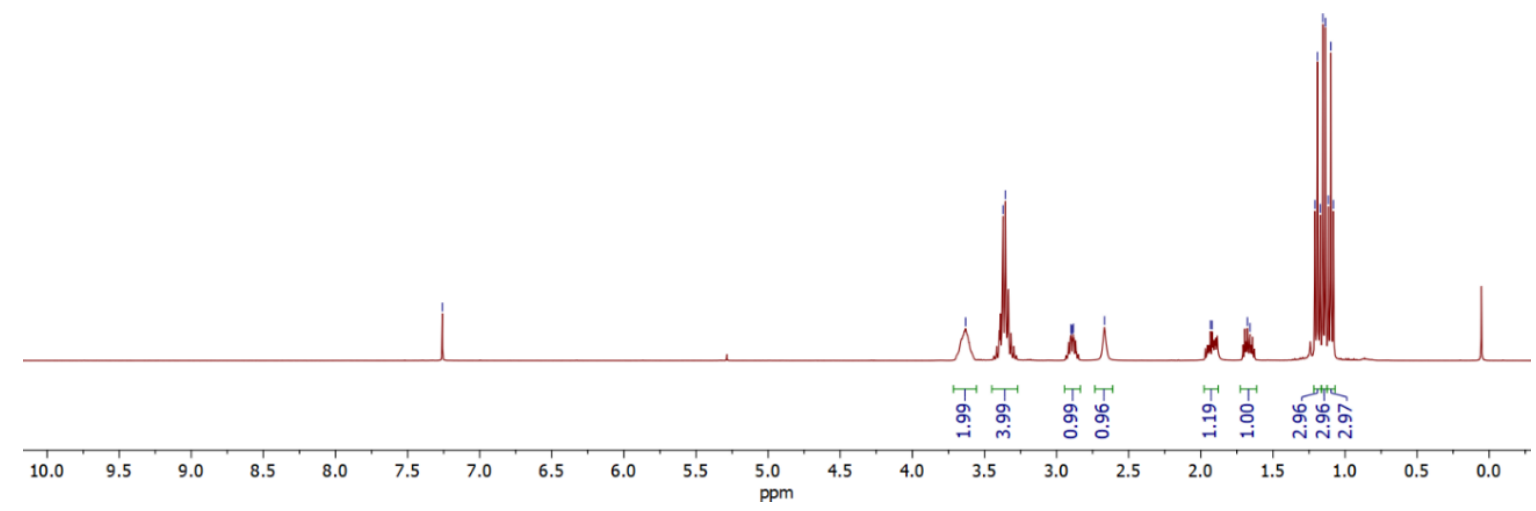

${ }^{13} \mathrm{C}\left\{{ }^{1} \mathrm{H}\right\}$ NMR $\left(\mathrm{CDCl}_{3}, 100.6 \mathrm{MHz}\right)$

早

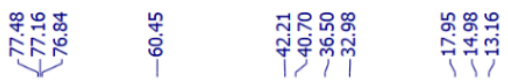<smiles>[Y20]C(CCO)C(=O)N(CC)CC</smiles>

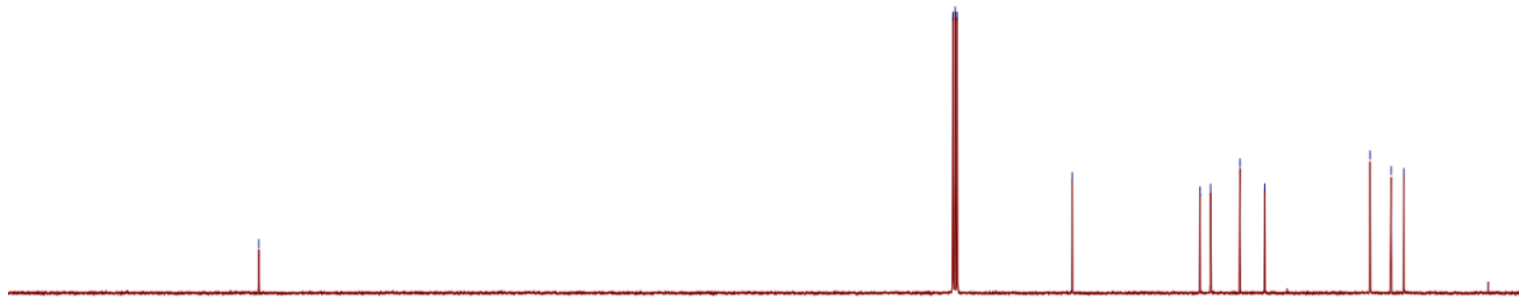

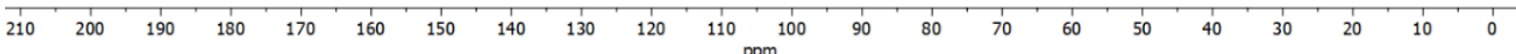



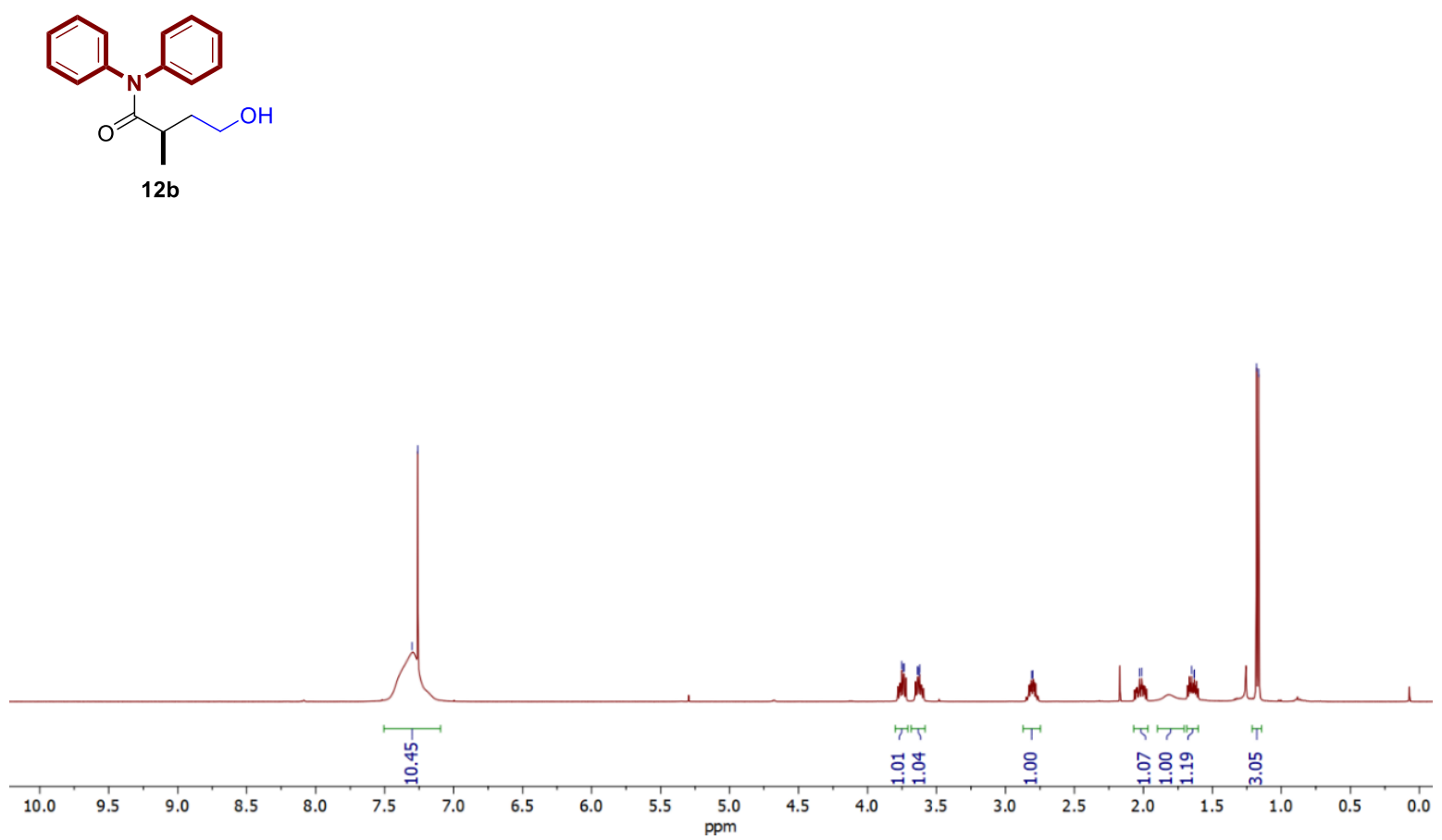

${ }^{13} \mathrm{C}\left\{{ }^{1} \mathrm{H}\right\} \mathrm{NMR}\left(\mathrm{CDCl}_{3}, 100.6 \mathrm{MHz}\right)$
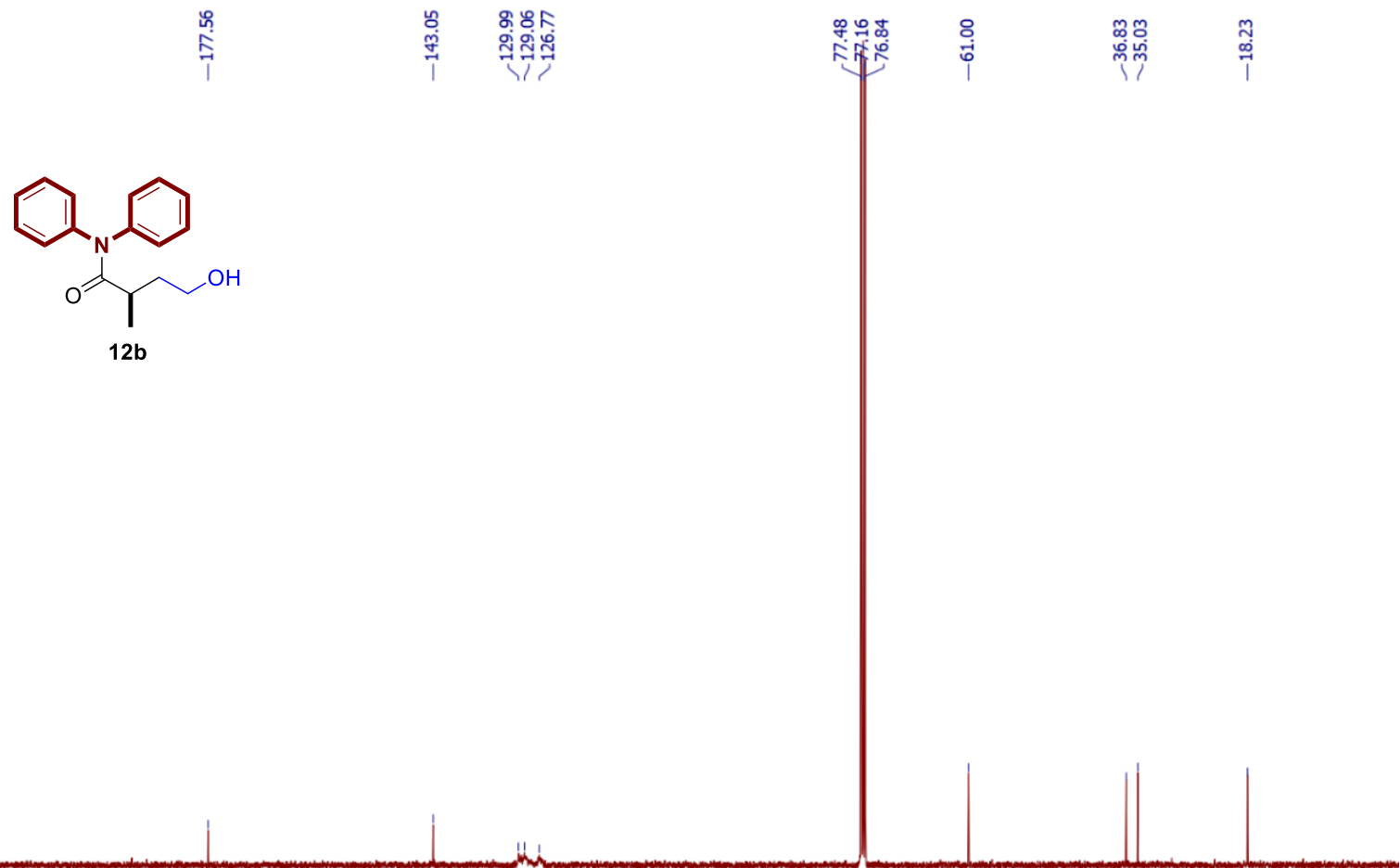

210

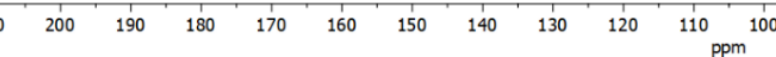


${ }^{1} \mathrm{H} \mathrm{NMR}\left(\mathrm{CDCl}_{3}, 400 \mathrm{MHz}\right)$<smiles>[Y20]C(CCO)C(=O)N(C(C)C)C(C)C</smiles>

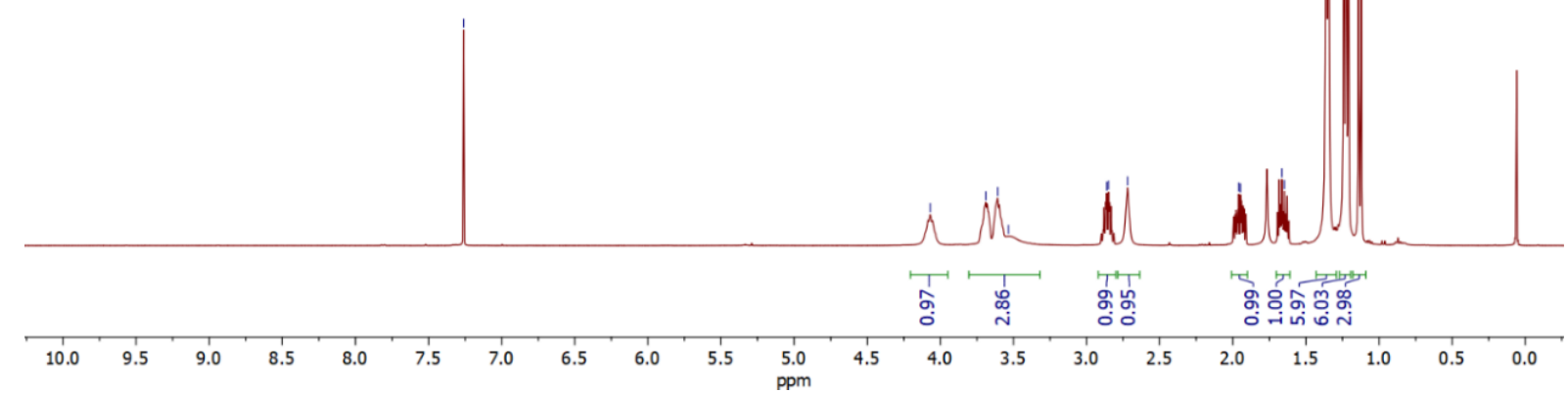

${ }^{13} \mathrm{C}\left\{{ }^{1} \mathrm{H}\right\} \operatorname{NMR}\left(\mathrm{CDCl}_{3}, 100.6 \mathrm{MHz}\right)$<smiles>P=[Te]</smiles><smiles>CC1C(C)N(C(C)C)C1C</smiles><smiles>[Y20]C(CCO)C(C)=O</smiles>

12c 
${ }^{1} \mathrm{H} \mathrm{NMR}\left(\mathrm{CDCl}_{3}, 400 \mathrm{MHz}\right)$

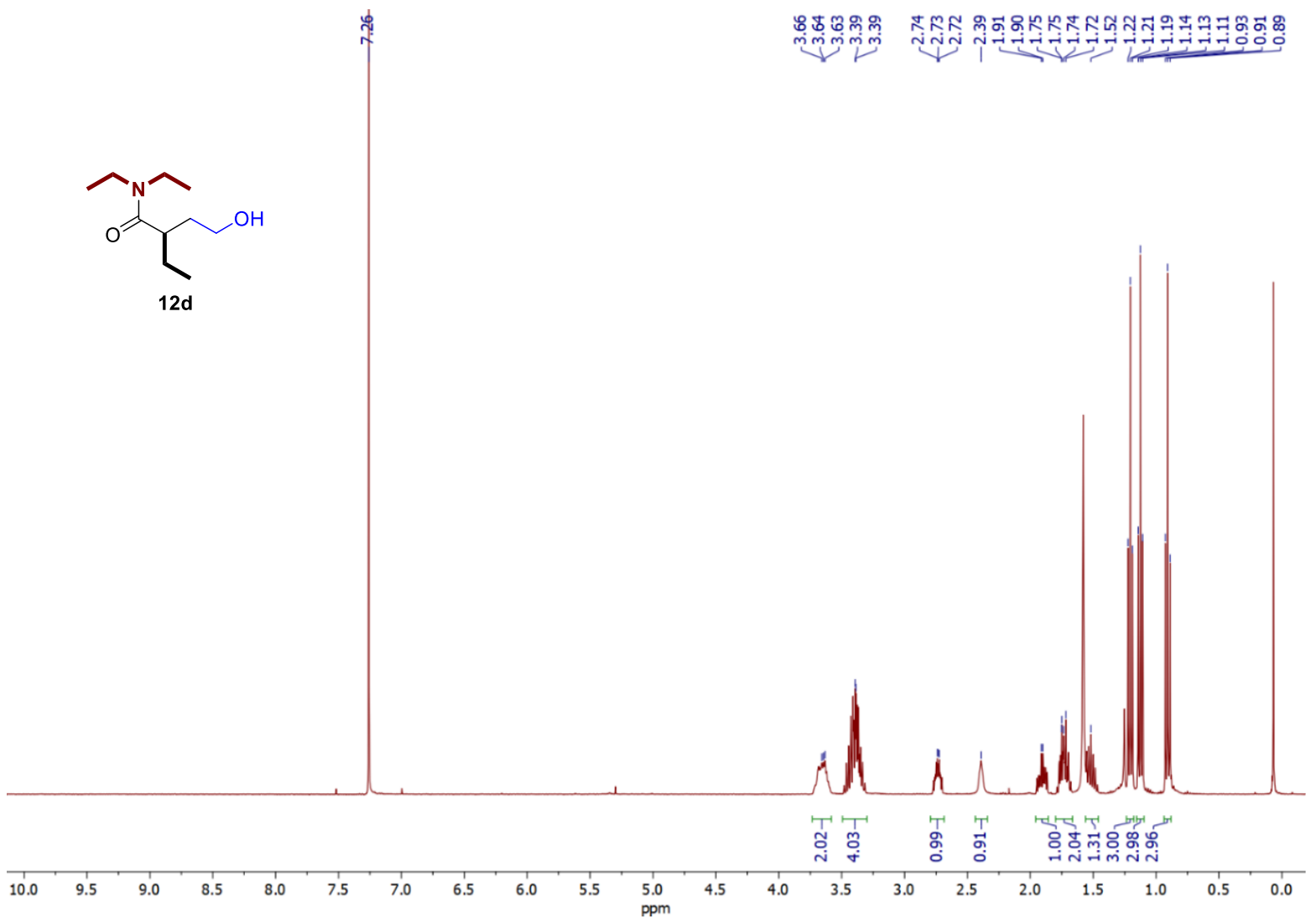

${ }^{13} \mathrm{C}\left\{{ }^{1} \mathrm{H}\right\} \operatorname{NMR}\left(\mathrm{CDCl}_{3}, 100.6 \mathrm{MHz}\right)$

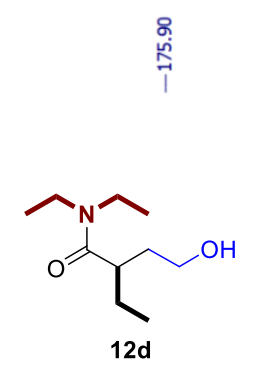


${ }^{1} \mathrm{H} \mathrm{NMR}\left(\mathrm{CDCl}_{3}, 400 \mathrm{MHz}\right)$

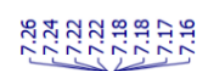

ஜ

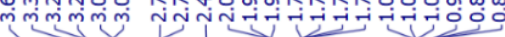

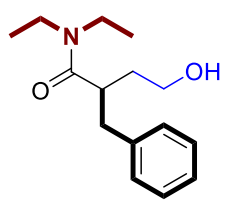

$12 \mathrm{e}$

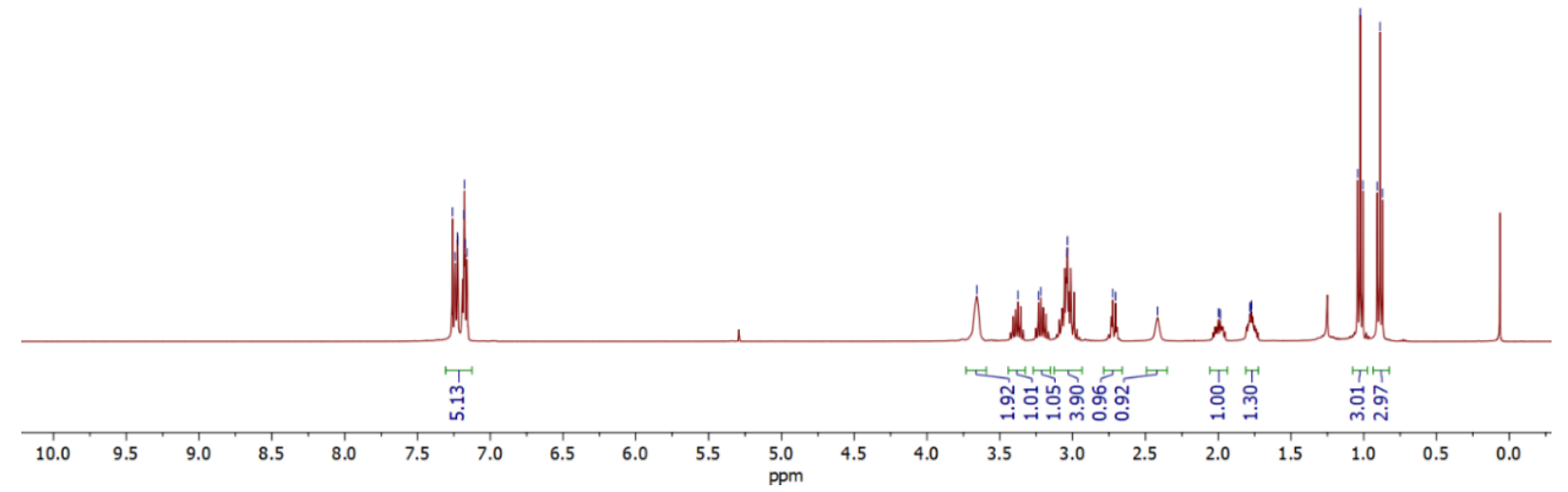

${ }^{13} \mathrm{C}\left\{{ }^{1} \mathrm{H}\right\} \operatorname{NMR}\left(\mathrm{CDCl}_{3}, 100.6 \mathrm{MHz}\right)$

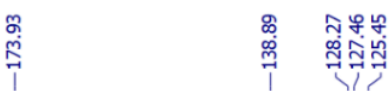

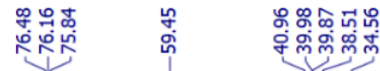

ติำ

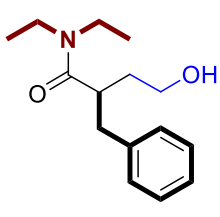

$12 \mathrm{e}$

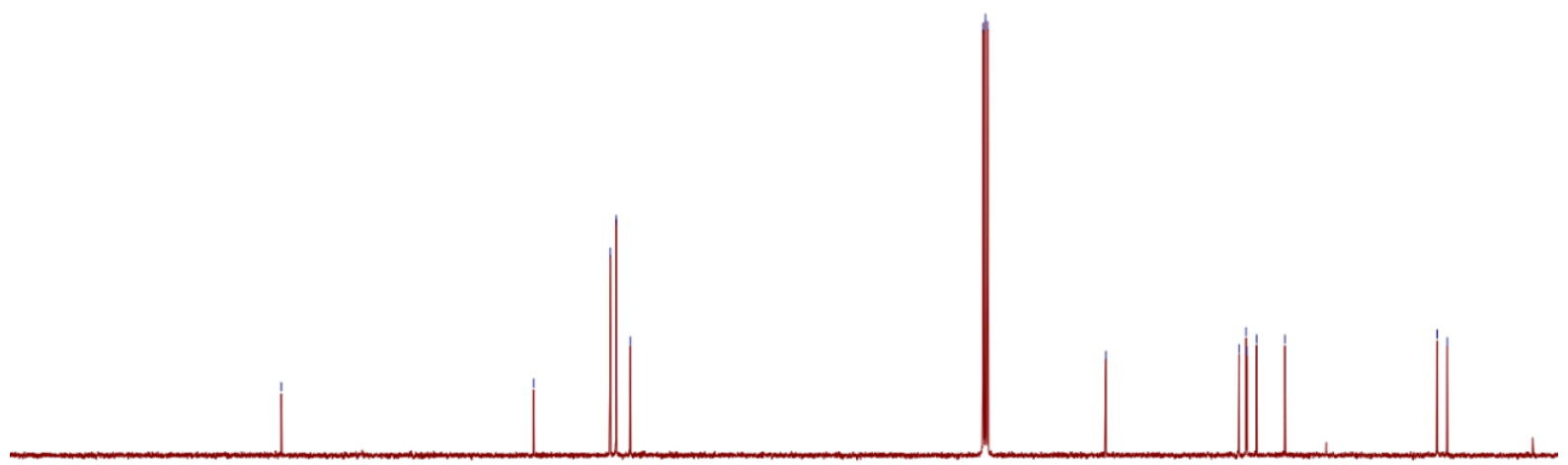

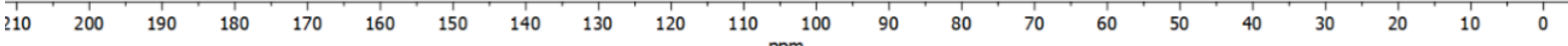


${ }^{1} \mathrm{H} \mathrm{NMR}\left(\mathrm{CDCl}_{3}, 400 \mathrm{MHz}\right)$

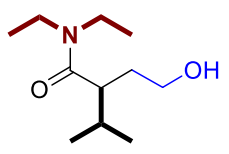

$12 f$

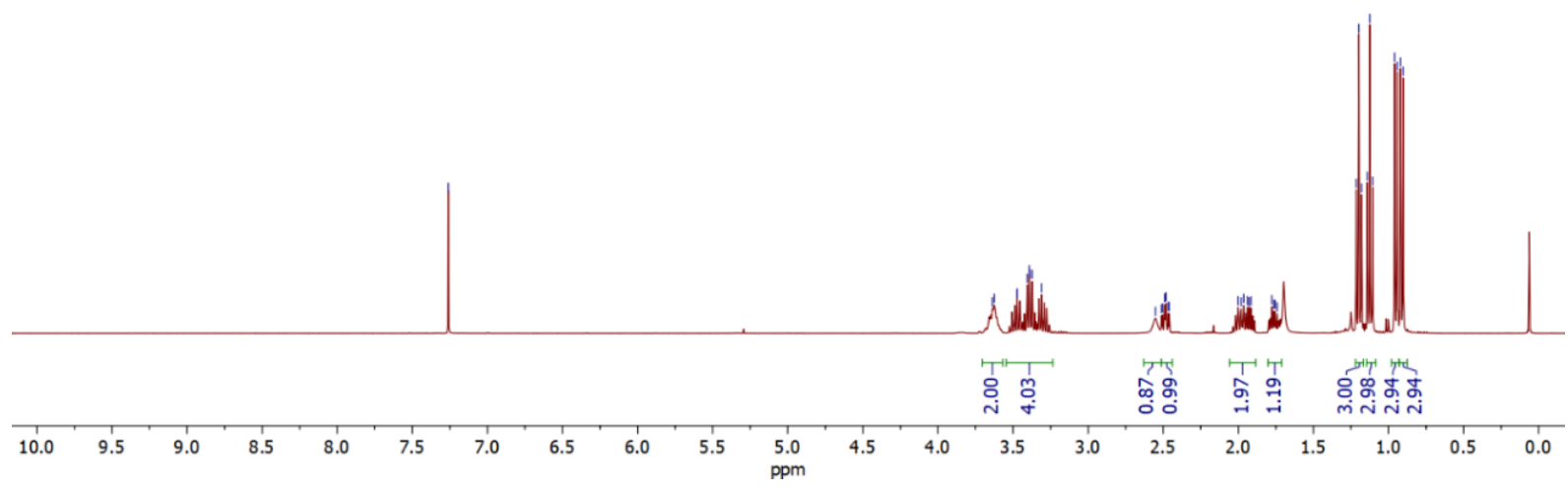

${ }^{13} \mathrm{C}\left\{{ }^{1} \mathrm{H}\right\} \operatorname{NMR}\left(\mathrm{CDCl}_{3}, 100.6 \mathrm{MHz}\right)$

$$
\text { กิ }
$$

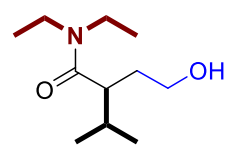

$12 f$ 
${ }^{1} \mathrm{H} \mathrm{NMR}\left(\mathrm{CDCl}_{3}, 400 \mathrm{MHz}\right)$

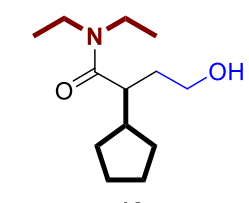

$12 \mathrm{~g}$

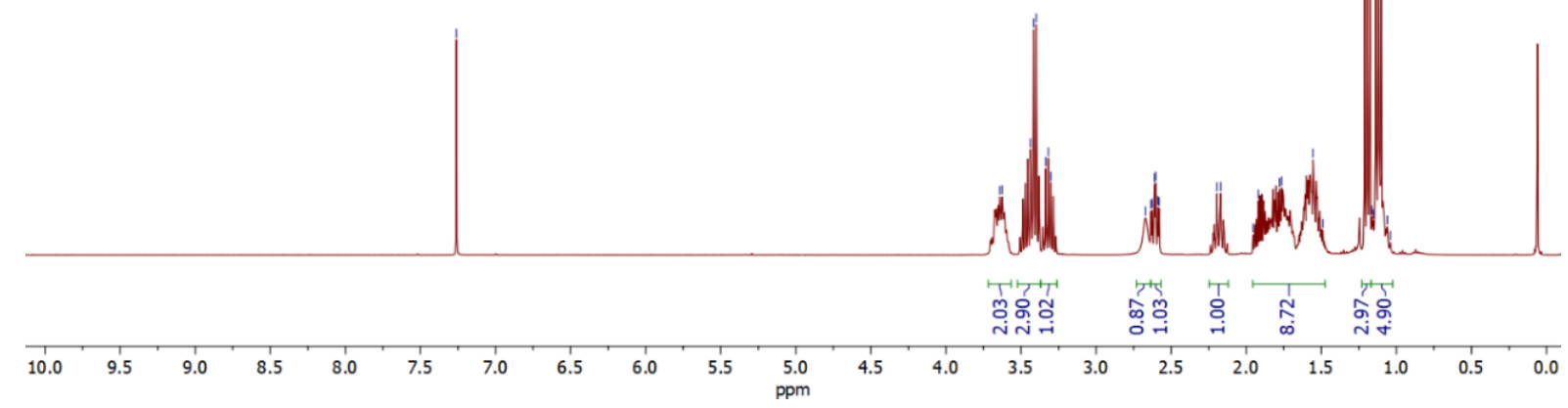

${ }^{13} \mathrm{C}\left\{{ }^{1} \mathrm{H}\right\} \mathrm{NMR}\left(\mathrm{CDCl}_{3}, 100.6 \mathrm{MHz}\right)$

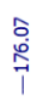

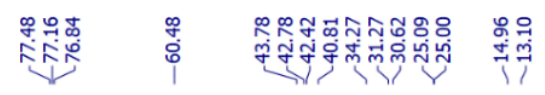

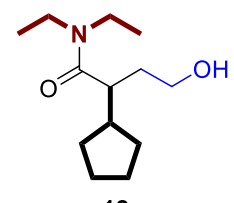

$12 \mathrm{~g}$

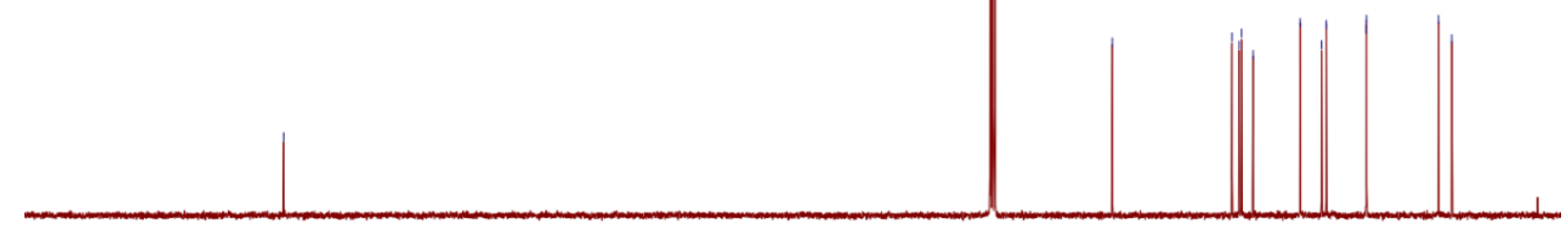

210

$200 \quad 190$

$190 \quad 180$

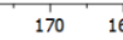

150

$110 \quad 100$

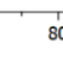

70

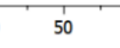


${ }^{1} \mathrm{H} \mathrm{NMR}\left(\mathrm{CDCl}_{3}, 400 \mathrm{MHz}\right)$

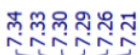

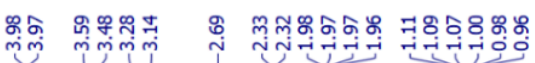

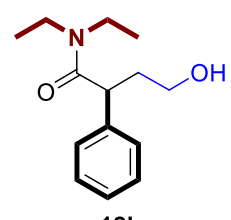

$12 \mathrm{~h}$

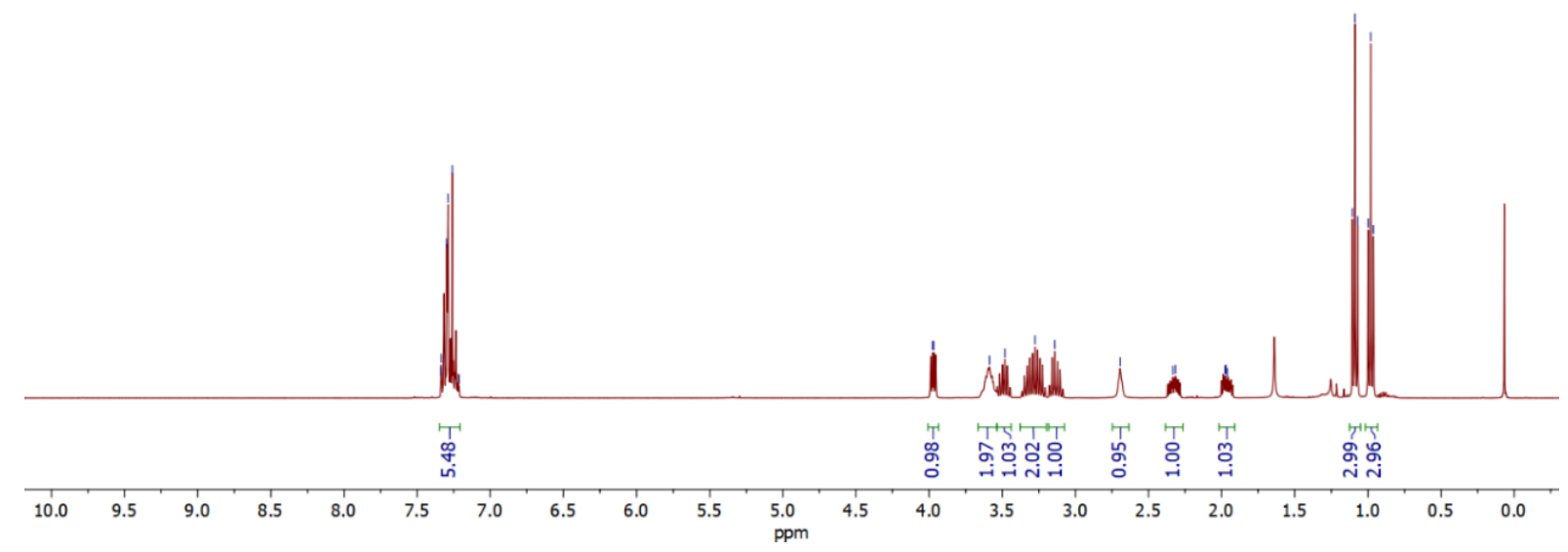

${ }^{13} \mathrm{C}\left\{{ }^{1} \mathrm{H}\right\} \mathrm{NMR}\left(\mathrm{CDCl}_{3}, 100.6 \mathrm{MHz}\right)$

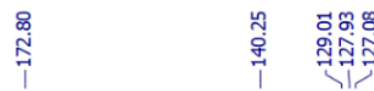

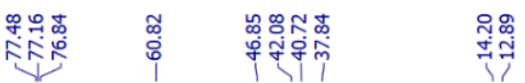<smiles>CCN(CC)C(=O)C(CCO)C1=CC=CCC1</smiles>

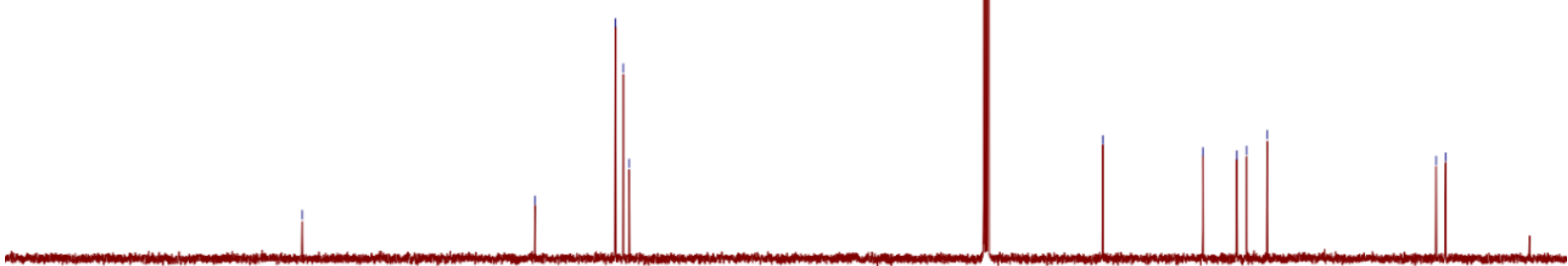

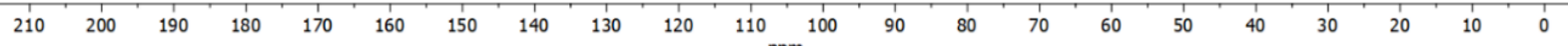


${ }^{1} \mathrm{H} \mathrm{NMR}\left(\mathrm{CDCl}_{3}, 400 \mathrm{MHz}\right)$
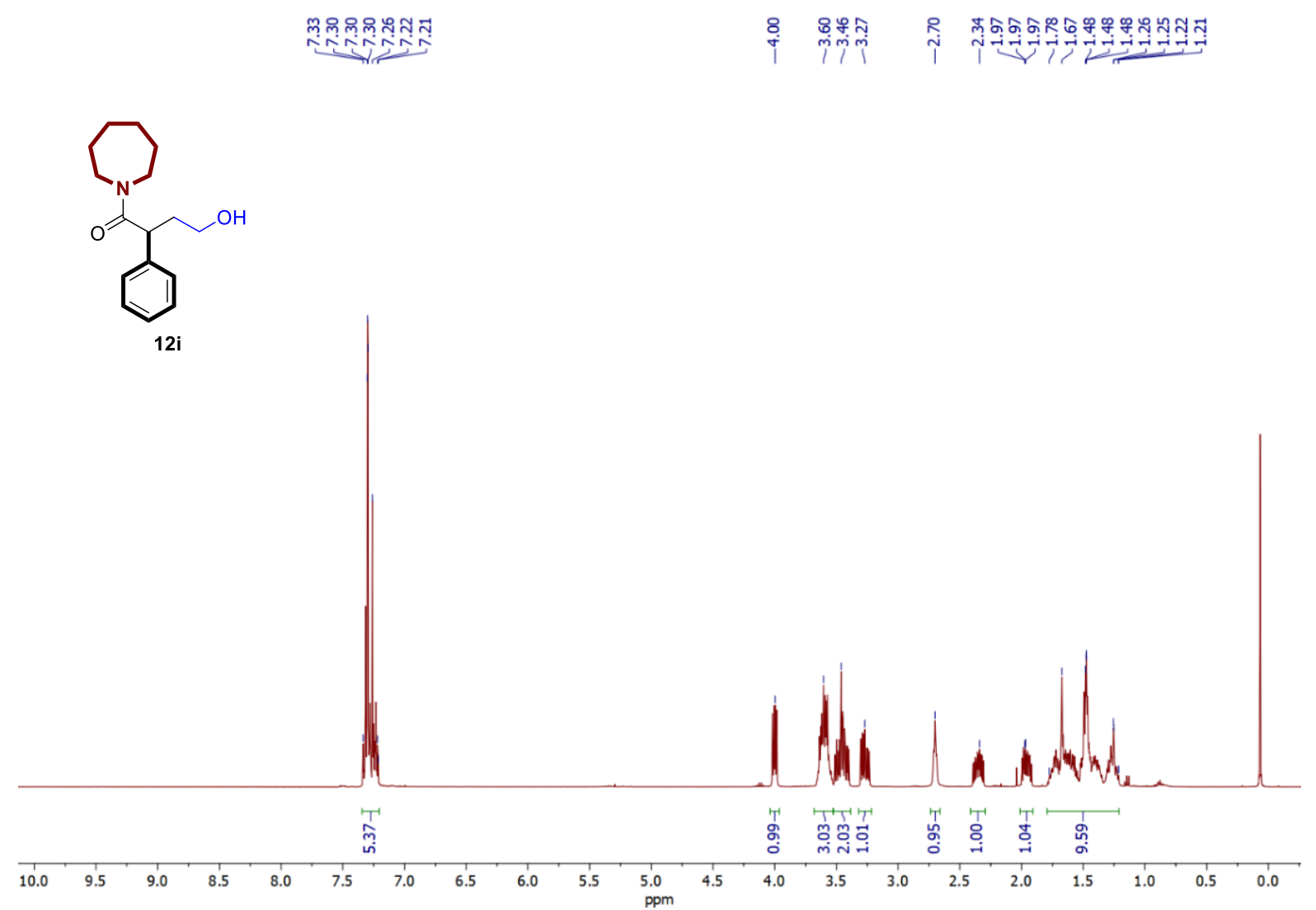

${ }^{13} \mathrm{C}\left\{{ }^{1} \mathrm{H}\right\}$ NMR $\left(\mathrm{CDCl}_{3}, 100.6 \mathrm{MHz}\right)$
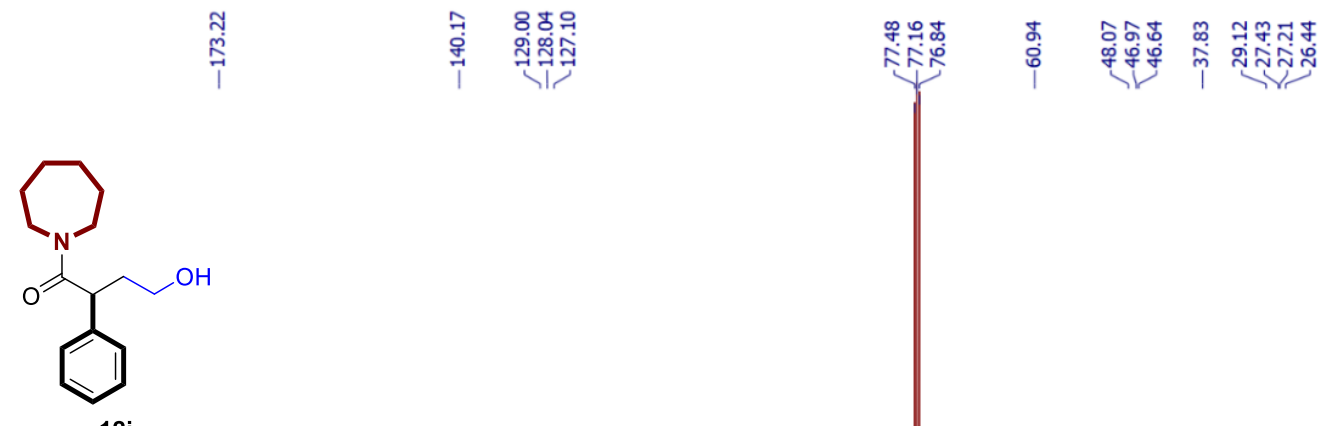

$12 i$

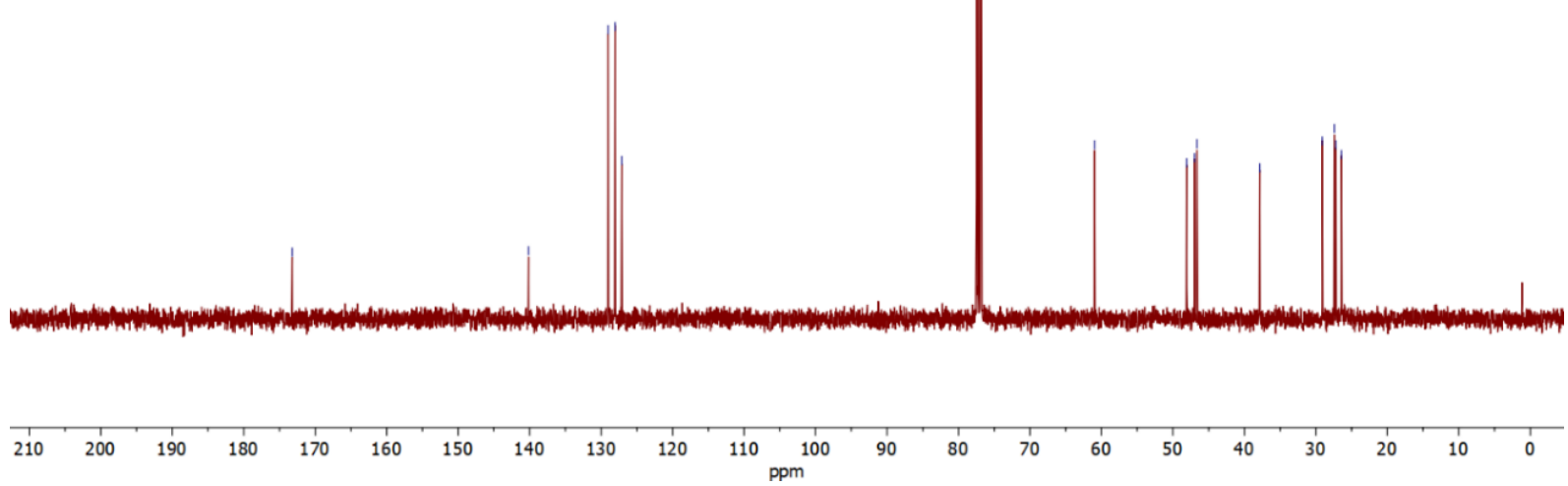




\section{e. $\alpha$-alkyl-y-aminobutyric amides}

${ }^{1} \mathrm{H} \mathrm{NMR}\left(\mathrm{CDCl}_{3}, 400 \mathrm{MHz}\right)$<smiles>CCN(CC)C(=O)C(C)CCN1CCOCC1</smiles>

$4 a$

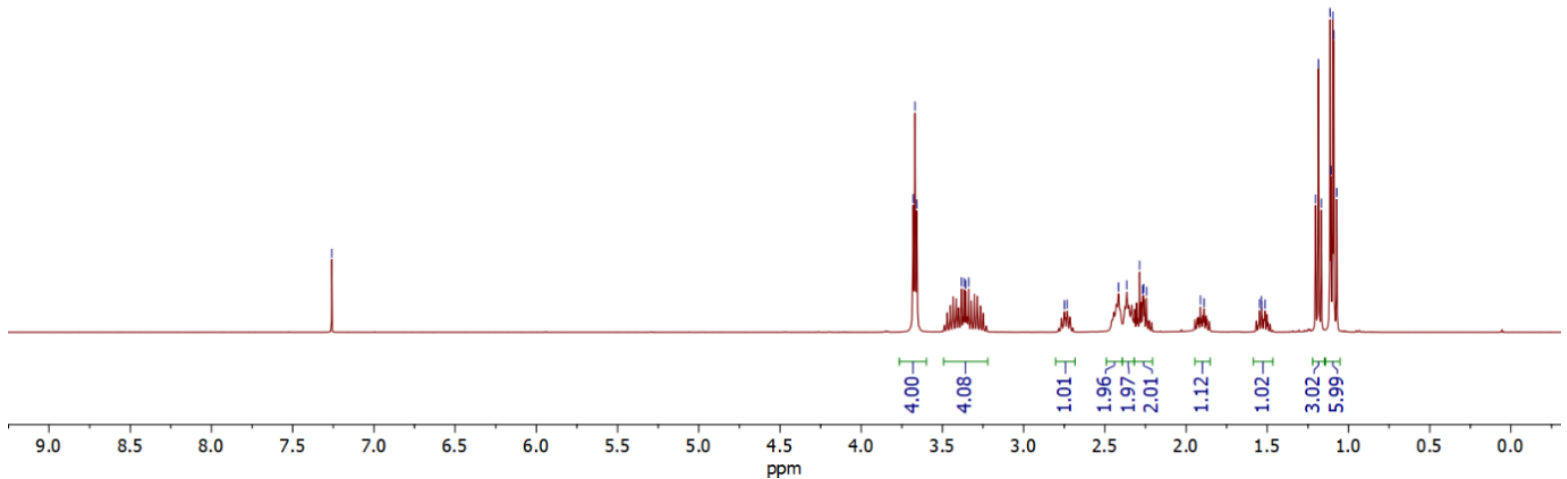

${ }^{13} \mathrm{C}\left\{{ }^{1} \mathrm{H}\right\} \mathrm{NMR}\left(\mathrm{CDCl}_{3}, 100.6 \mathrm{MHz}\right)$

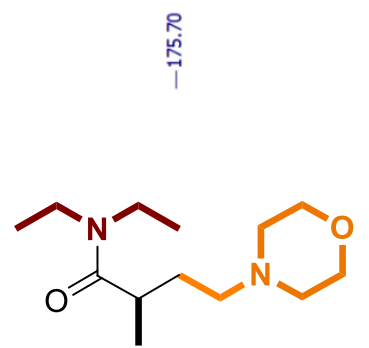

$4 a$

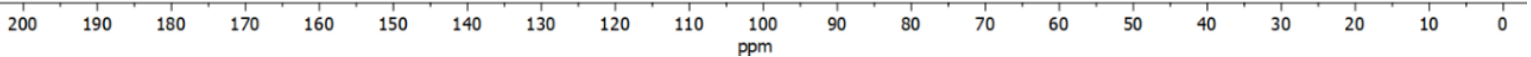


${ }^{1} \mathrm{H} \mathrm{NMR}\left(\mathrm{CDCl}_{3}, 400 \mathrm{MHz}\right)$

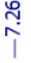<smiles>CCN(CC)C(=O)C(C)CCN1CCN(C(=O)OC(C)(C)C)CC1</smiles>

4b

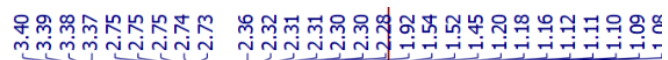

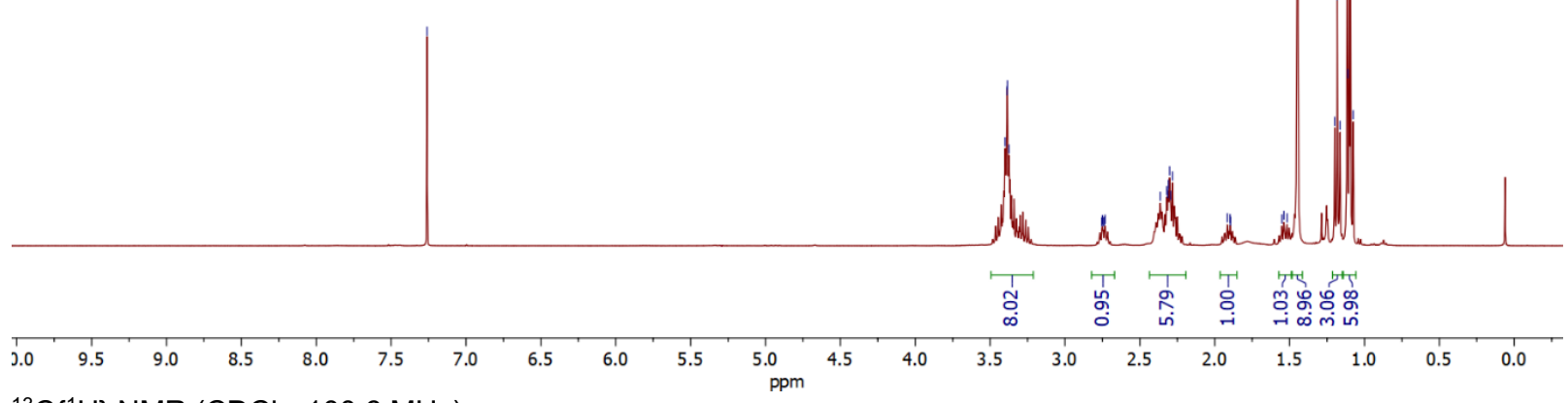

${ }^{13} \mathrm{C}\left\{{ }^{1} \mathrm{H}\right\} \operatorname{NMR}\left(\mathrm{CDCl}_{3}, 100.6 \mathrm{MHz}\right)$

永哭

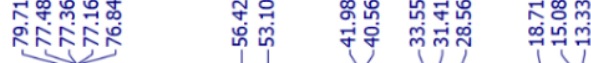<smiles>CCN(CC)C(=O)C(C)CCN1CCN(C(=O)OC(C)(C)C)CC1</smiles>

$4 b$

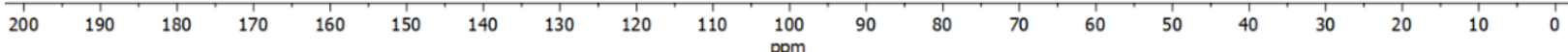


${ }^{1} \mathrm{H} \mathrm{NMR}\left(\mathrm{CDCl}_{3}, 400 \mathrm{MHz}\right)$

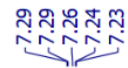<smiles>CCN(CC)C(=O)C(C)CCN(C)Cc1ccccc1</smiles>

4c

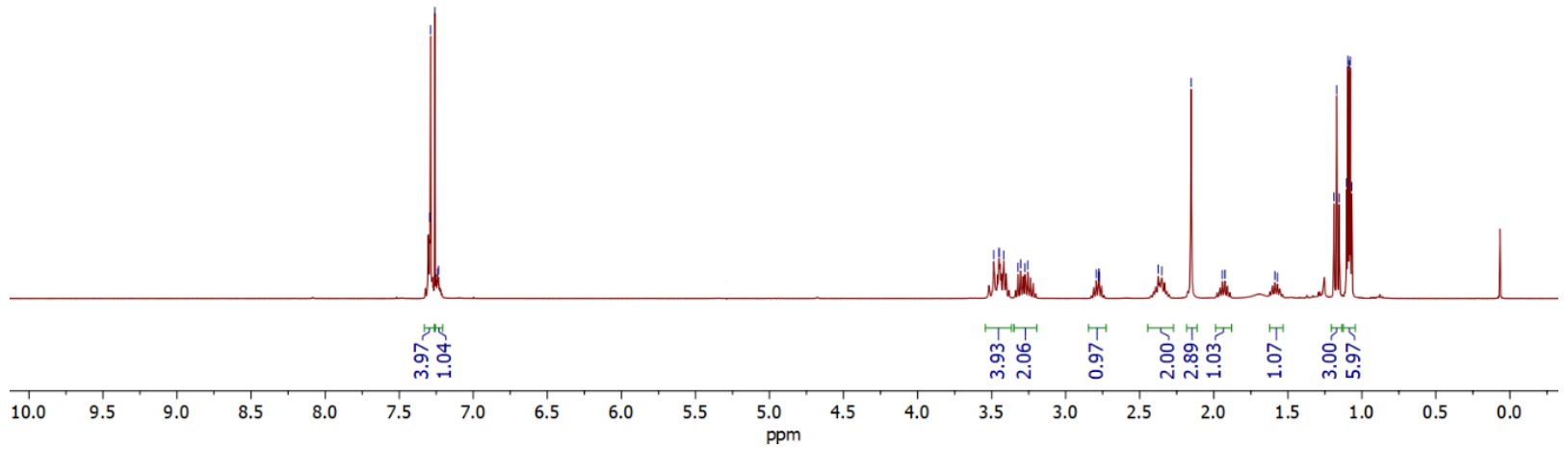

${ }^{13} \mathrm{C}\left\{{ }^{1} \mathrm{H}\right\} \operatorname{NMR}\left(\mathrm{CDCl}_{3}, 100.6 \mathrm{MHz}\right)$

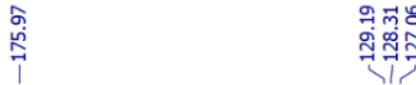

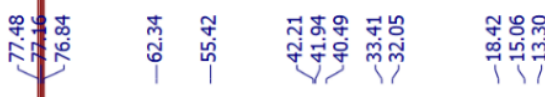<smiles>CCN(CC)C(=O)C(C)CCN(C)Cc1ccccc1</smiles>

$4 c$

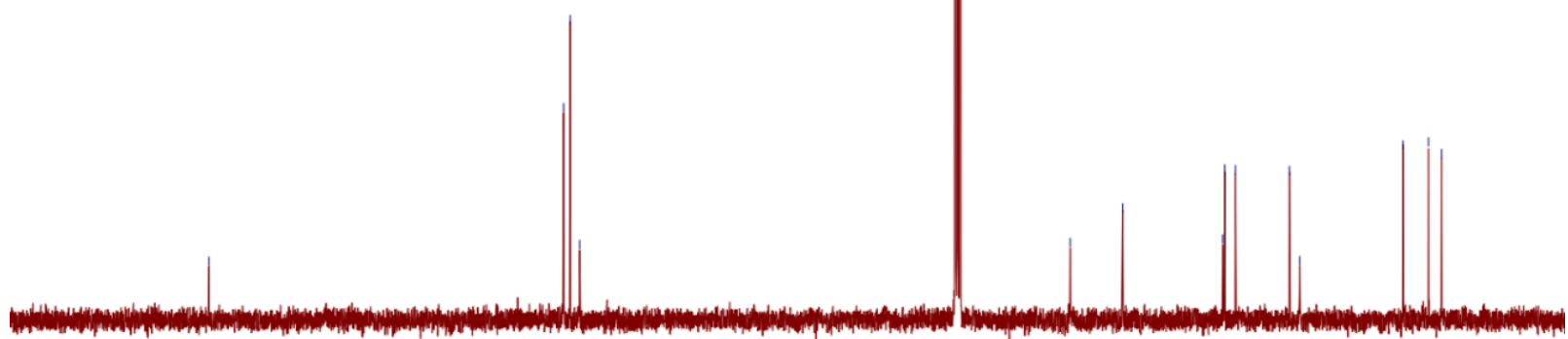

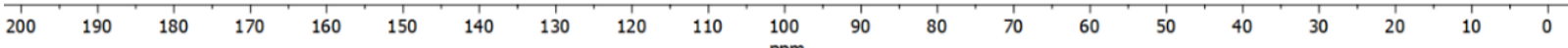


<smiles>CCN(CC)C(=O)C(C)CCN1CCN(c2ccccc2OC)CC1</smiles>

4d

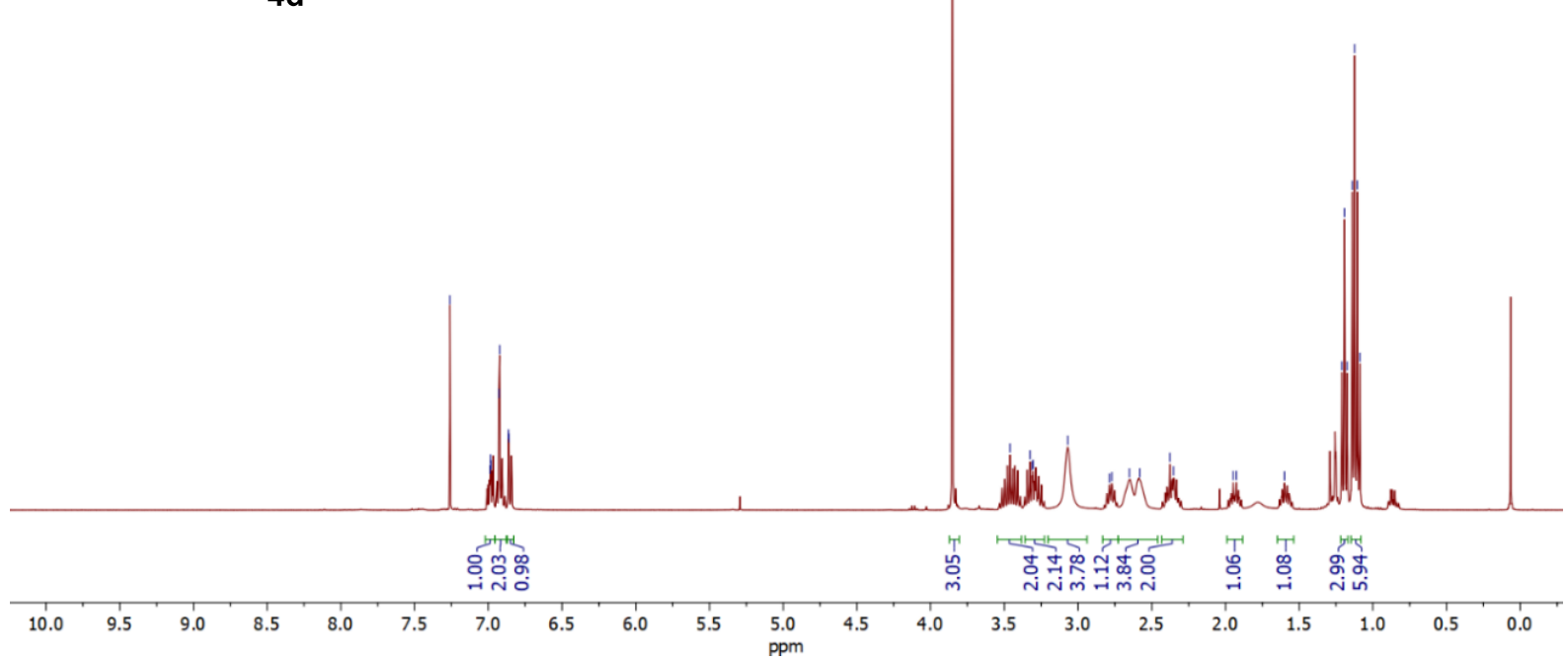
${ }^{13} \mathrm{C}\left\{{ }^{1} \mathrm{H}\right\} \mathrm{NMR}\left(\mathrm{CDCl}_{3}, 100.6 \mathrm{MHz}\right)$

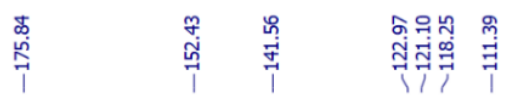

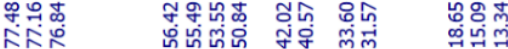

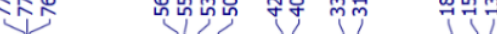<smiles>CCN(CC)C(=O)C(C)CCN1CCN(c2ccccc2OC)CC1</smiles>

4d 
${ }^{1} \mathrm{H} \mathrm{NMR}\left(\mathrm{CDCl}_{3}, 400 \mathrm{MHz}\right)$

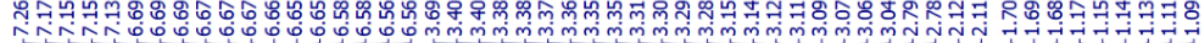<smiles>CCN(CC)C(=O)C(C)CCNc1ccccc1</smiles>

$4 e$

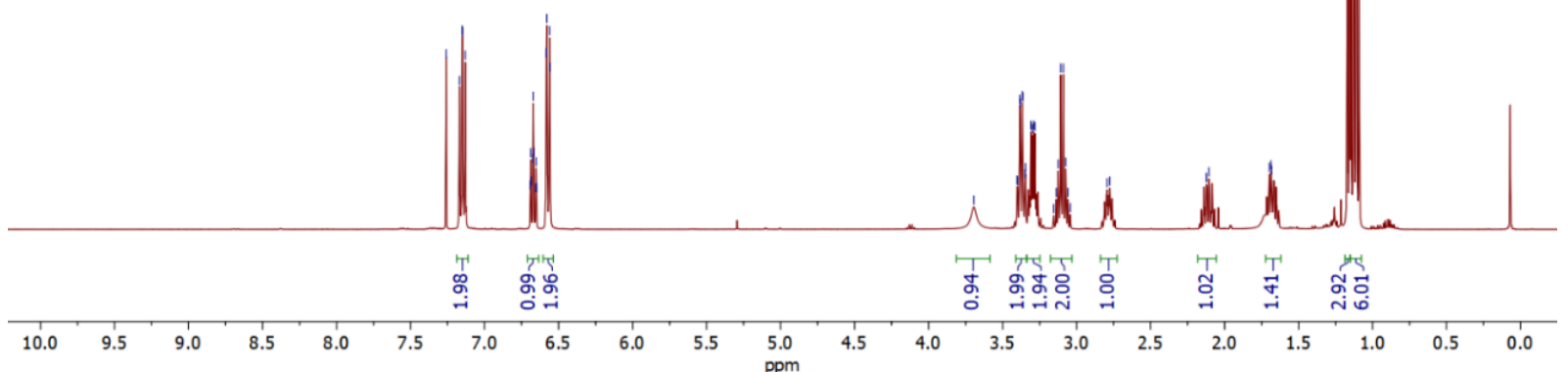
${ }^{13} \mathrm{C}\left\{{ }^{1} \mathrm{H}\right\} \operatorname{NMR}\left(\mathrm{CDCl}_{3}, 100.6 \mathrm{MHz}\right)$

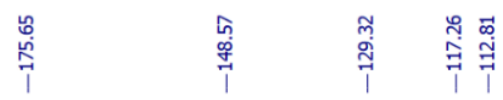

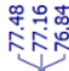

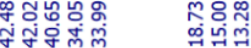<smiles>CCN(CC)C(=O)C(C)CCNc1ccccc1</smiles>

$4 e$

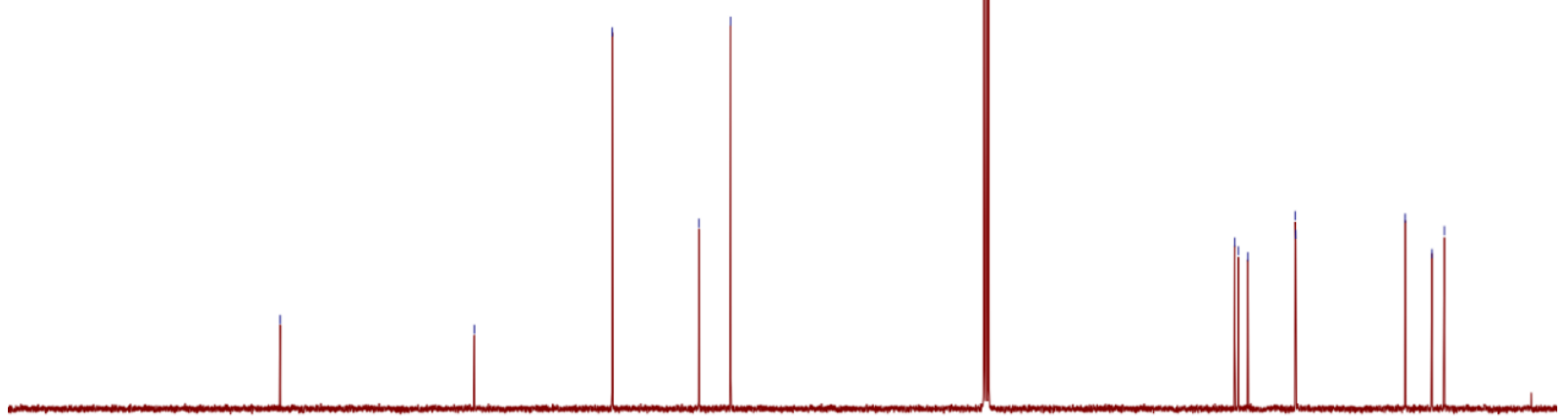

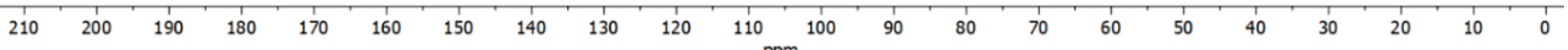


${ }^{1} \mathrm{H} \mathrm{NMR}\left(\mathrm{CDCl}_{3}, 400 \mathrm{MHz}\right)$<smiles>CC(CCN1CCOCC1)C(=O)C(=O)N(C(C)C)C(C)C</smiles>

$4 f$

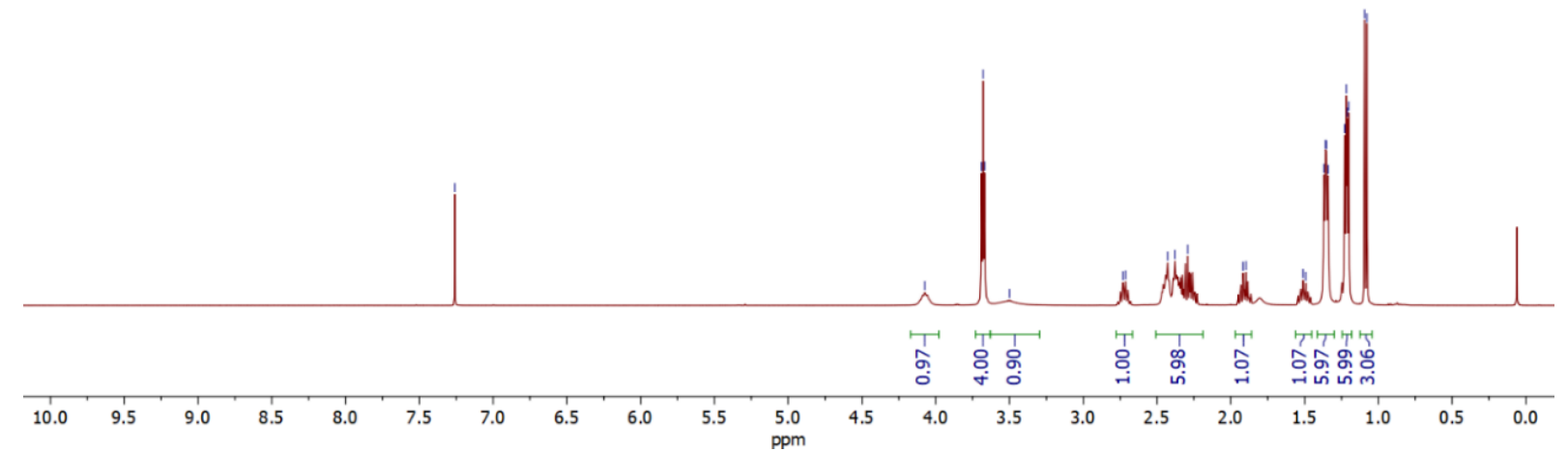
${ }^{13} \mathrm{C}\left\{{ }^{1} \mathrm{H}\right\}$ NMR $\left(\mathrm{CDCl}_{3}, 100.6 \mathrm{MHz}\right)$

趈

诲<smiles>CC(CCN1CCOCC1)C(=O)C(C(C)C)N(C(C)C)C(C)C</smiles>

$4 f$

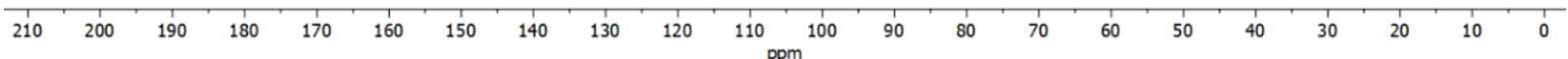


<smiles>CC(CCN1CCOCC1)C(=O)N(c1ccccc1)c1ccccc1</smiles>

$4 \mathrm{~g}$

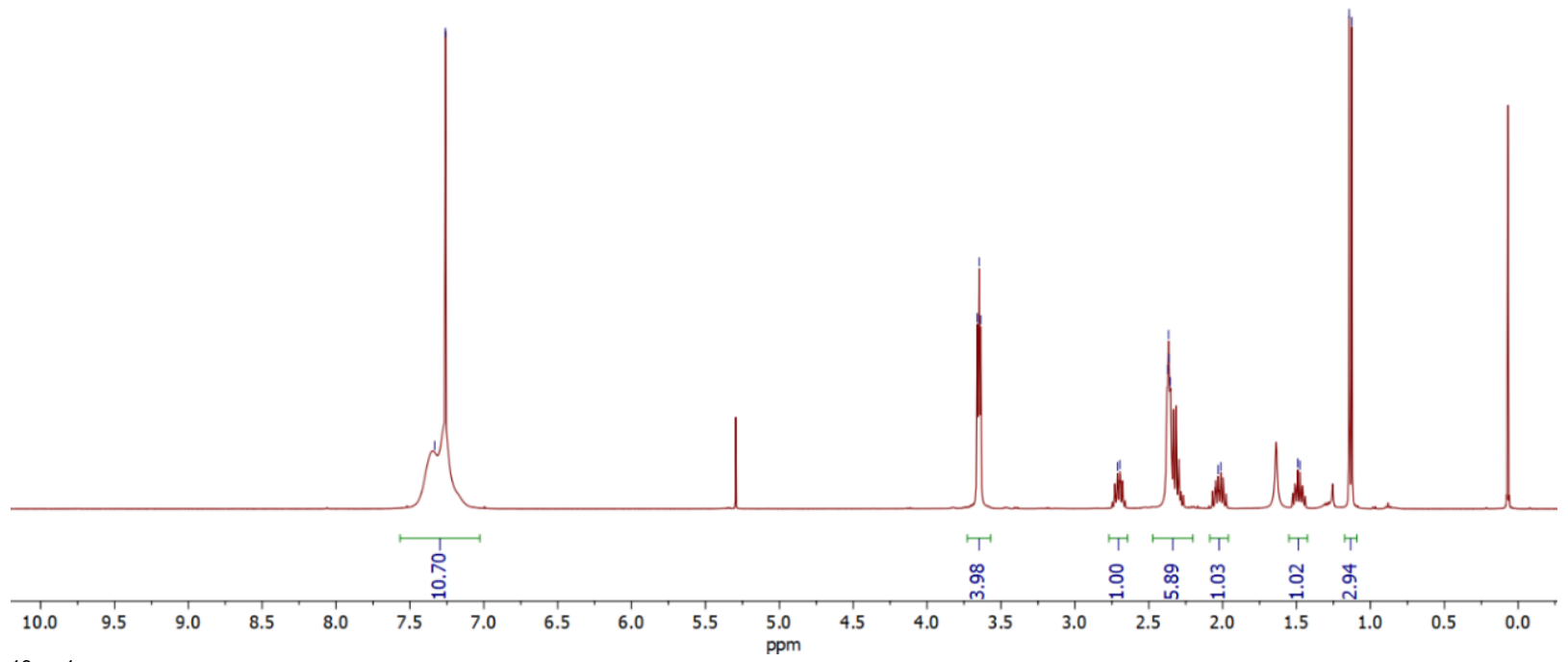

${ }^{13} \mathrm{C}\left\{{ }^{1} \mathrm{H}\right\} \operatorname{NMR}\left(\mathrm{CDCl}_{3}, 100.6 \mathrm{MHz}\right)$

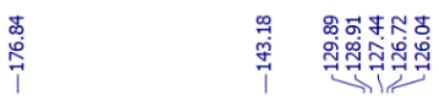

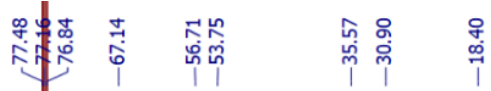<smiles>CC(CCN1CCOCC1)C(=O)N(c1ccccc1)c1ccccc1</smiles>

$4 \mathrm{~g}$

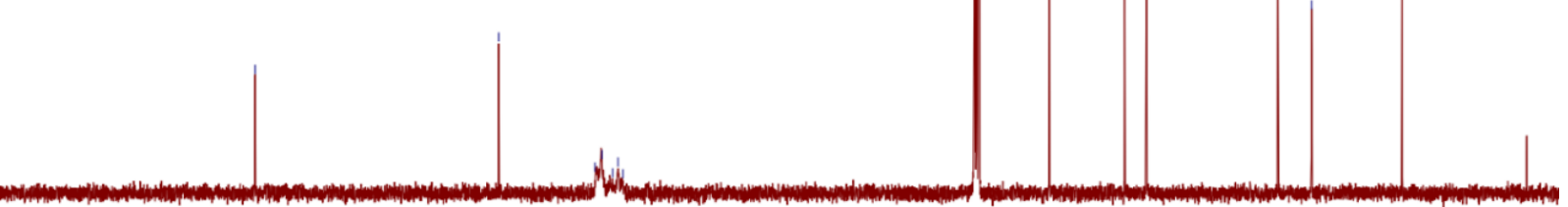

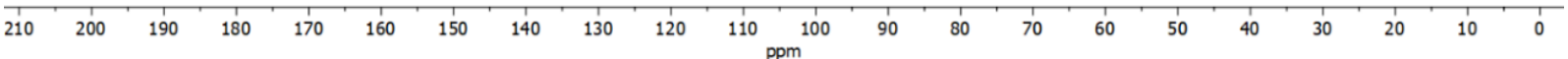


${ }^{1} \mathrm{H} \mathrm{NMR}\left(\mathrm{CDCl}_{3}, 400 \mathrm{MHz}\right)$<smiles>CCC(CCN1CCOCC1)C(=O)N(CC)CC</smiles>

4h

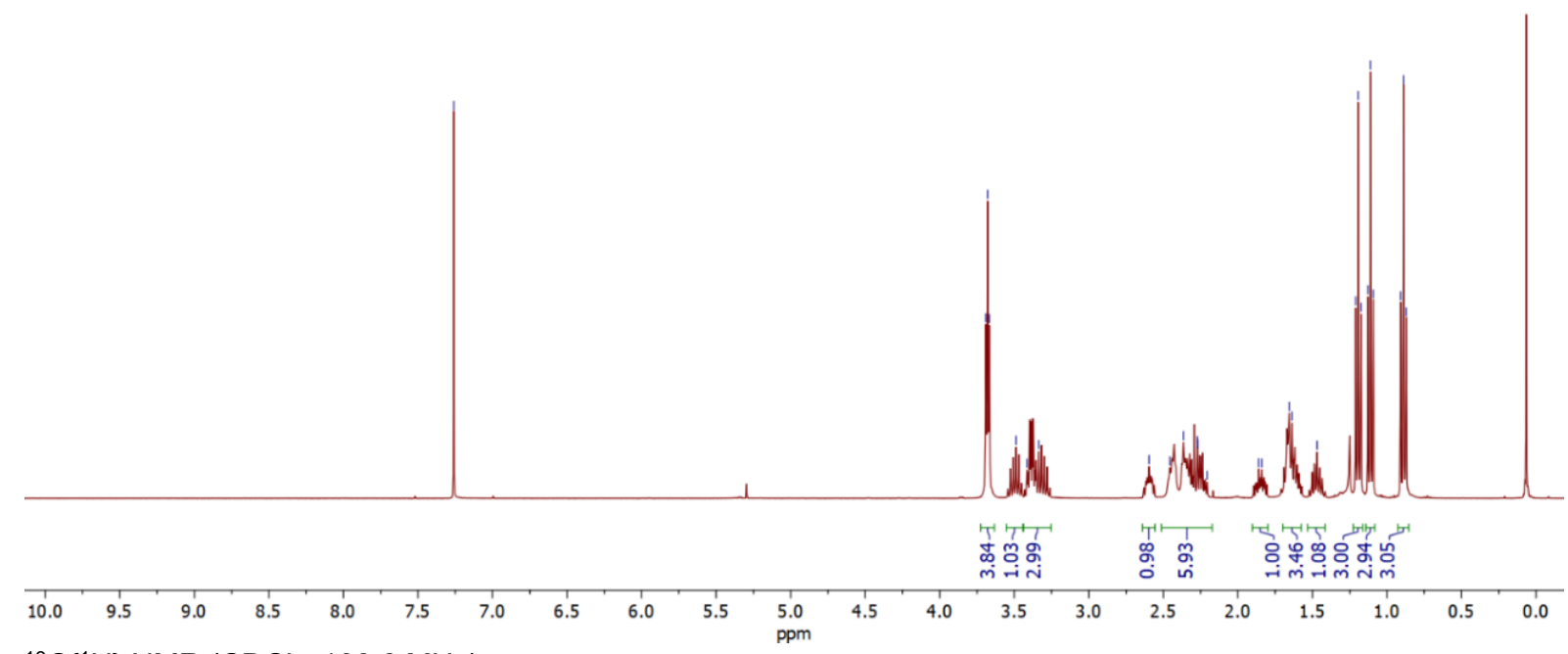

${ }^{13} \mathrm{C}\left\{{ }^{1} \mathrm{H}\right\} \mathrm{NMR}\left(\mathrm{CDCl}_{3}, 100.6 \mathrm{MHz}\right)$

药<smiles>CCC(CCN1CCOCC1)C(=O)N(CC)CC</smiles>

$4 \mathrm{~h}$

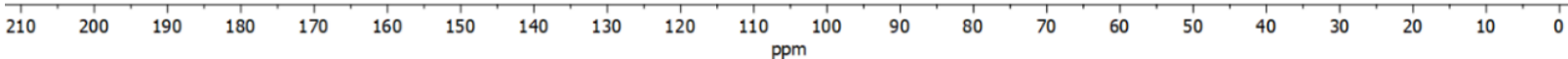


${ }^{1} \mathrm{H} \mathrm{NMR}\left(\mathrm{CDCl}_{3}, 400 \mathrm{MHz}\right)$

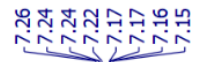<smiles>CCN(CC)C(=O)C(CCN1CCOCC1)Cc1ccccc1</smiles>

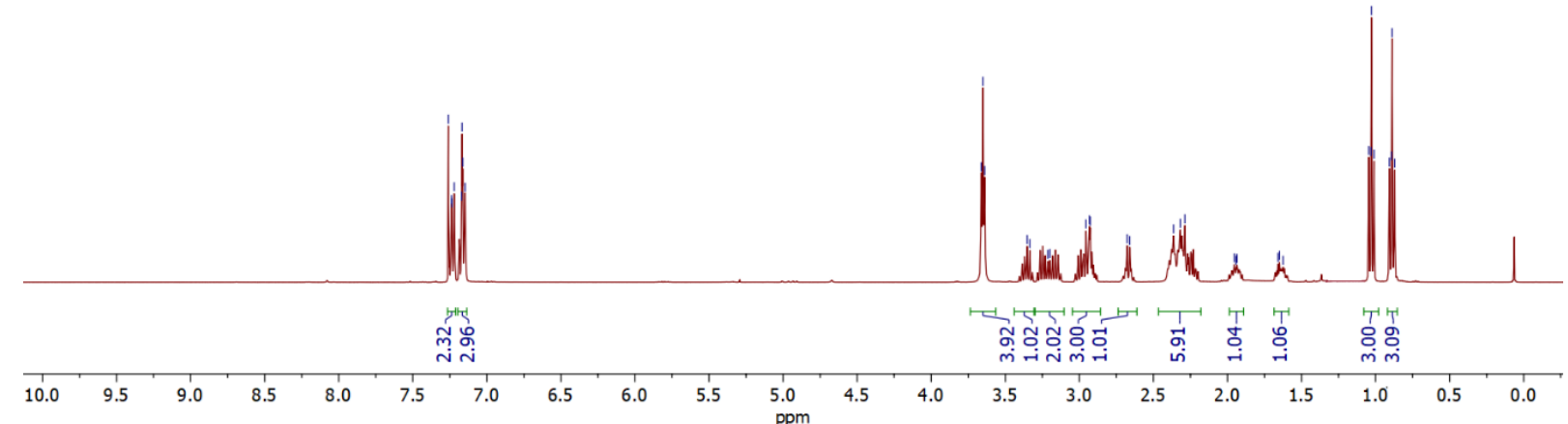

${ }^{13} \mathrm{C}\left\{{ }^{1} \mathrm{H}\right\}$ NMR $\left(\mathrm{CDCl}_{3}, 100.6 \mathrm{MHz}\right)$

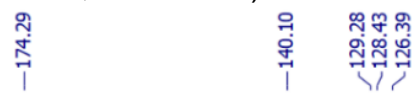<smiles>CCN(CC)C(=O)C(CCN1CCOCC1)Cc1ccccc1</smiles>

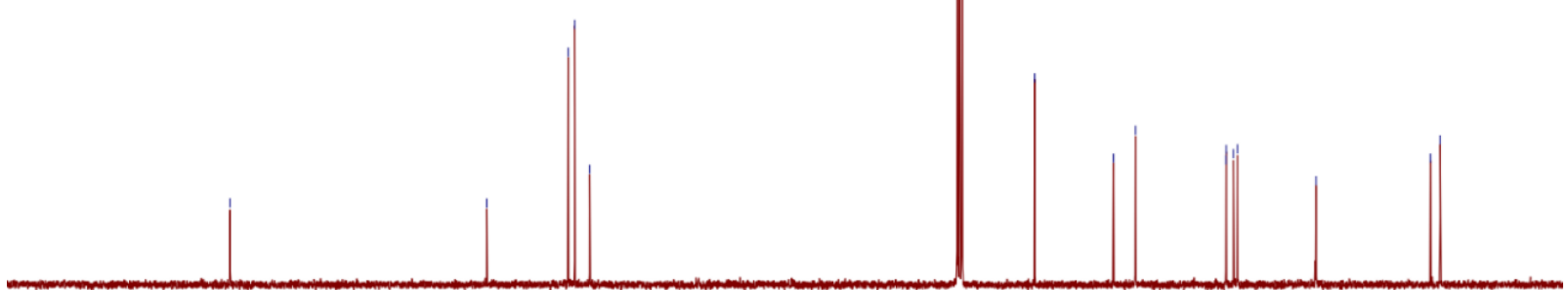


${ }^{1} \mathrm{H} \mathrm{NMR}\left(\mathrm{CDCl}_{3}, 400 \mathrm{MHz}\right)$

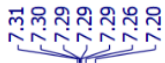

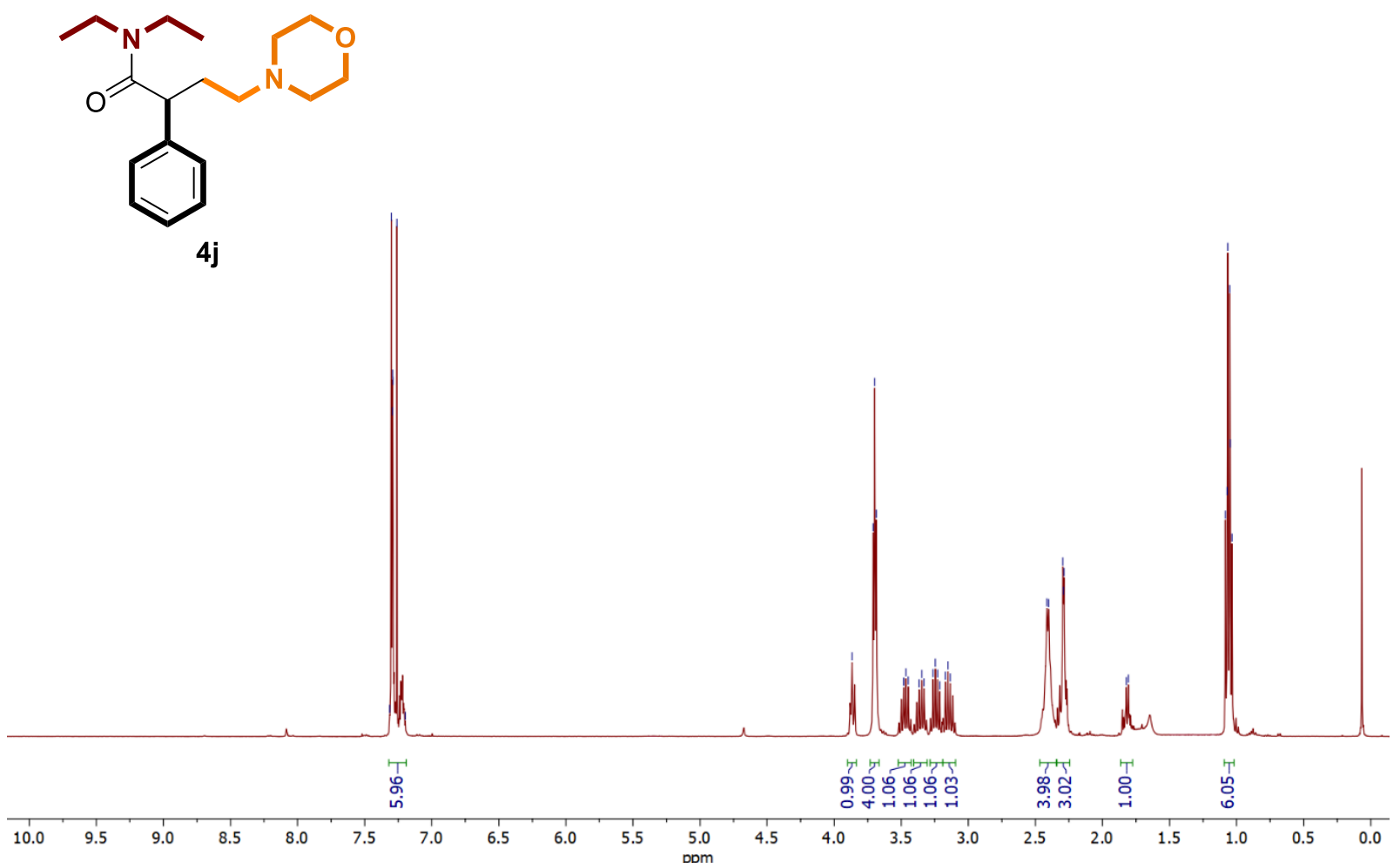

${ }^{13} \mathrm{C}\left\{{ }^{1} \mathrm{H}\right\} \mathrm{NMR}\left(\mathrm{CDCl}_{3}, 100.6 \mathrm{MHz}\right)$

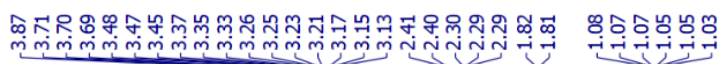

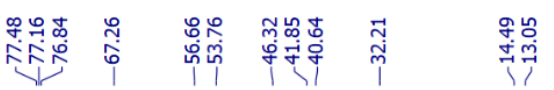<smiles>CCN(CC)C(=O)C(CCN1CCOCC1)c1ccccc1</smiles>

4j 
<smiles>O=C(C(CCN1CCOCC1)c1ccccc1)N1CCCCC1</smiles>

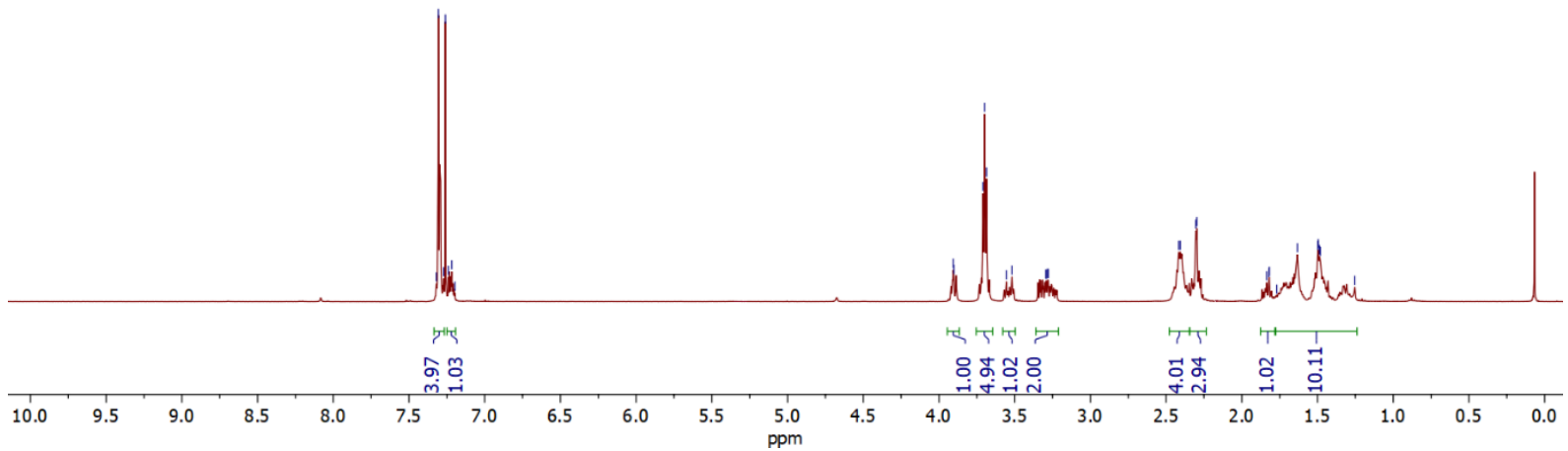
${ }^{13} \mathrm{C}\left\{{ }^{1} \mathrm{H}\right\} \mathrm{NMR}\left(\mathrm{CDCl}_{3}, 100.6 \mathrm{MHz}\right)$

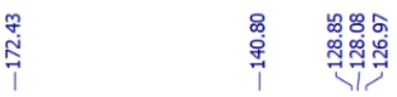<smiles>O=C(C(CCN1CCOCC1)c1ccccc1)N1CCCCCC1</smiles>

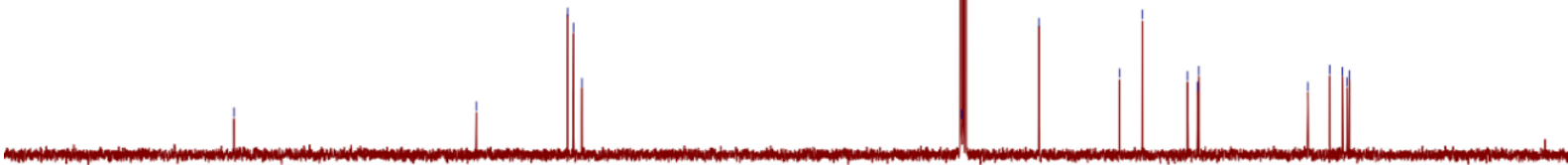

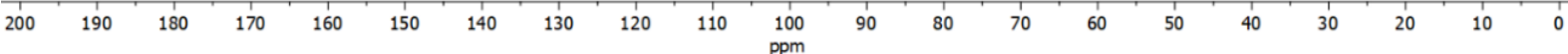


$\square_{N}$<smiles>CCC(=O)N1CCN(CCC(C(=O)NC)c2ccccc2)CC1</smiles>

4I

handrund

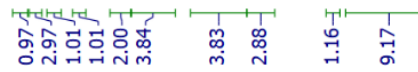

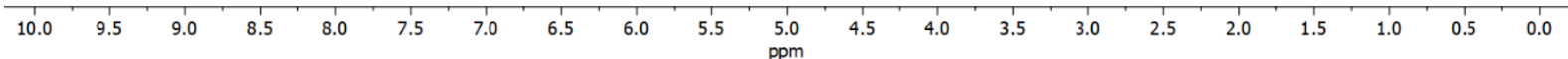
${ }^{13} \mathrm{C}\left\{{ }^{1} \mathrm{H}\right\} \mathrm{NMR}\left(\mathrm{CDCl}_{3}, 100.6 \mathrm{MHz}\right)$

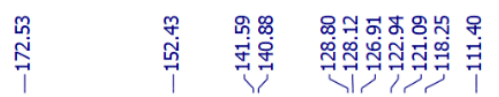

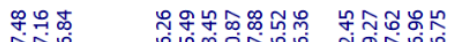

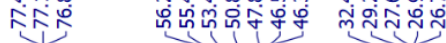<smiles>COc1ccccc1N1CCN(CCC(C(=O)N2CCCCCC2)c2ccccc2)CC1</smiles>

4

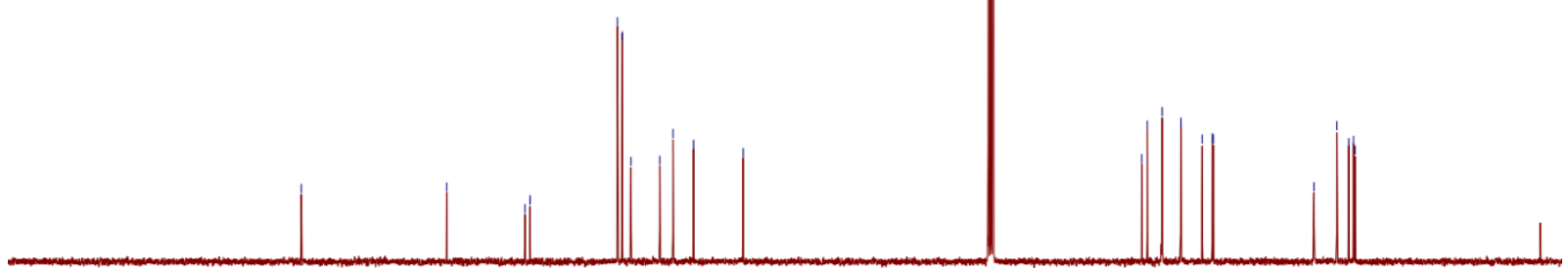

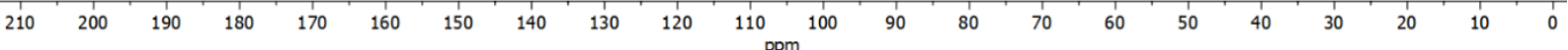




\section{f. Aminoalcohols 5-10}

${ }^{1} \mathrm{H} \mathrm{NMR}\left(\mathrm{CDCl}_{3}, 250 \mathrm{MHz}\right)$
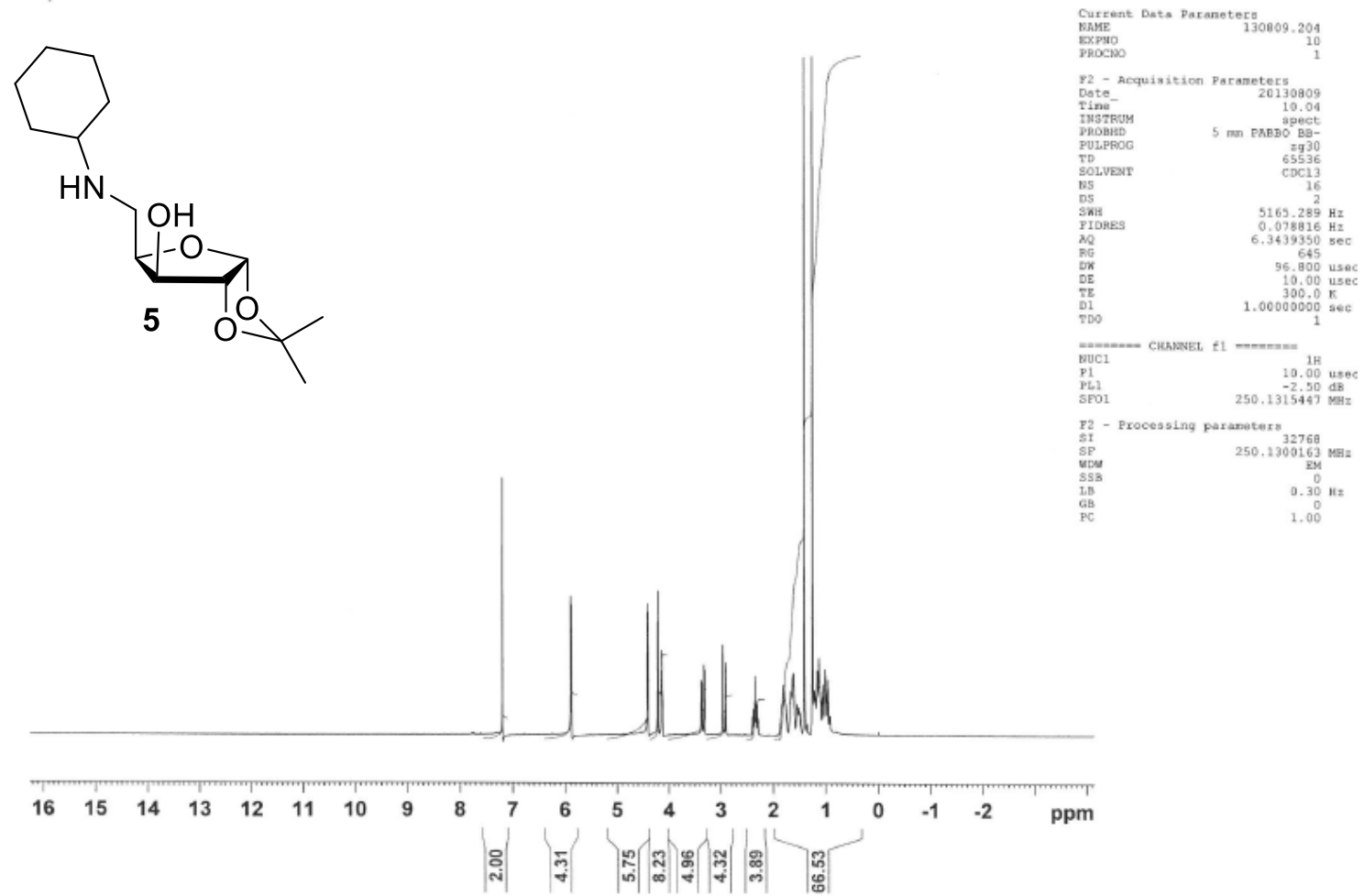

${ }^{13} \mathrm{C}\left\{{ }^{1} \mathrm{H}\right\}$ NMR $\left(\mathrm{CDCl}_{3}, 63 \mathrm{MHz}\right)$

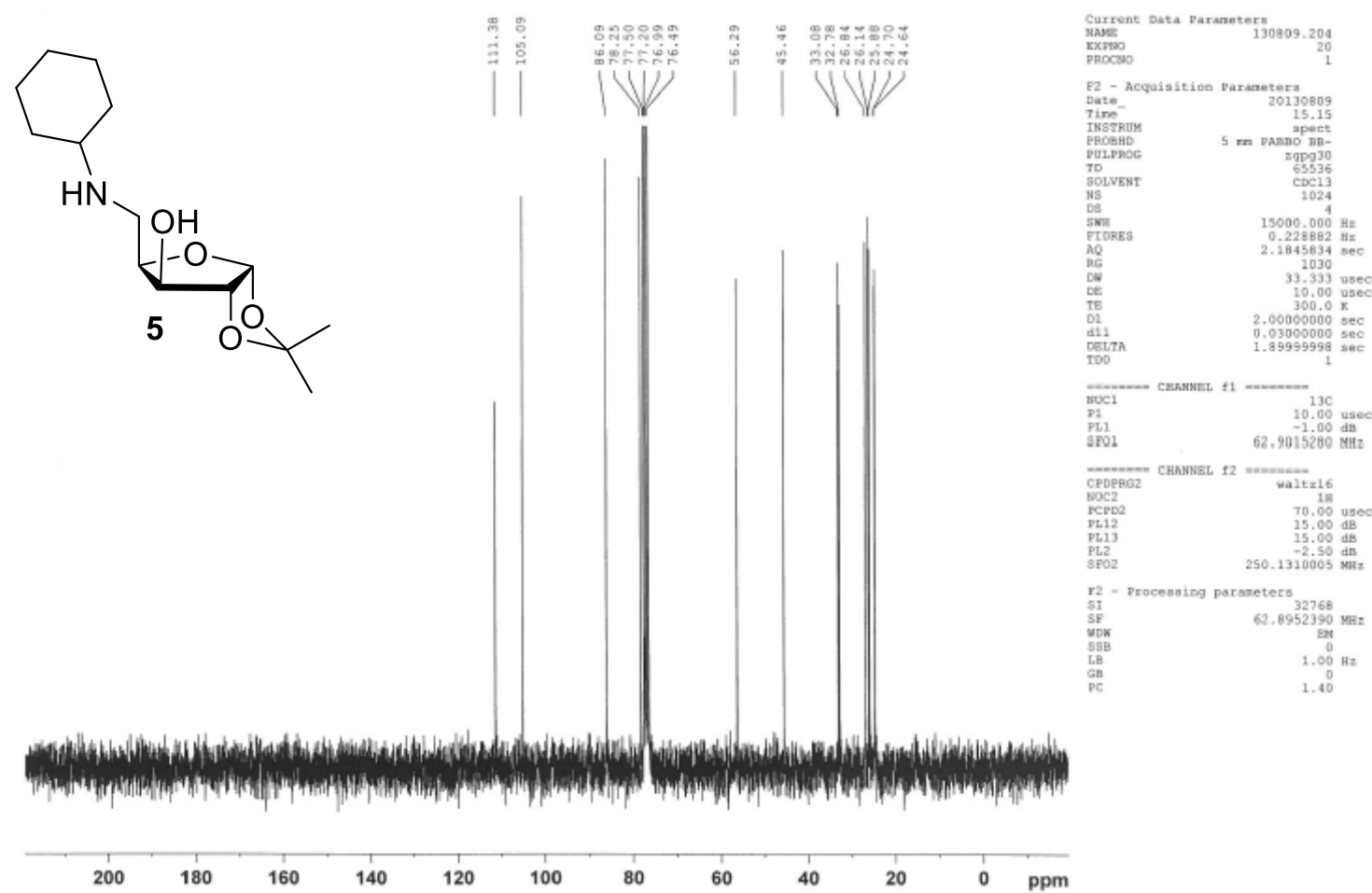


${ }^{1} \mathrm{H} \mathrm{NMR}\left(\mathrm{CDCl}_{3}, 400 \mathrm{MHz}\right)$

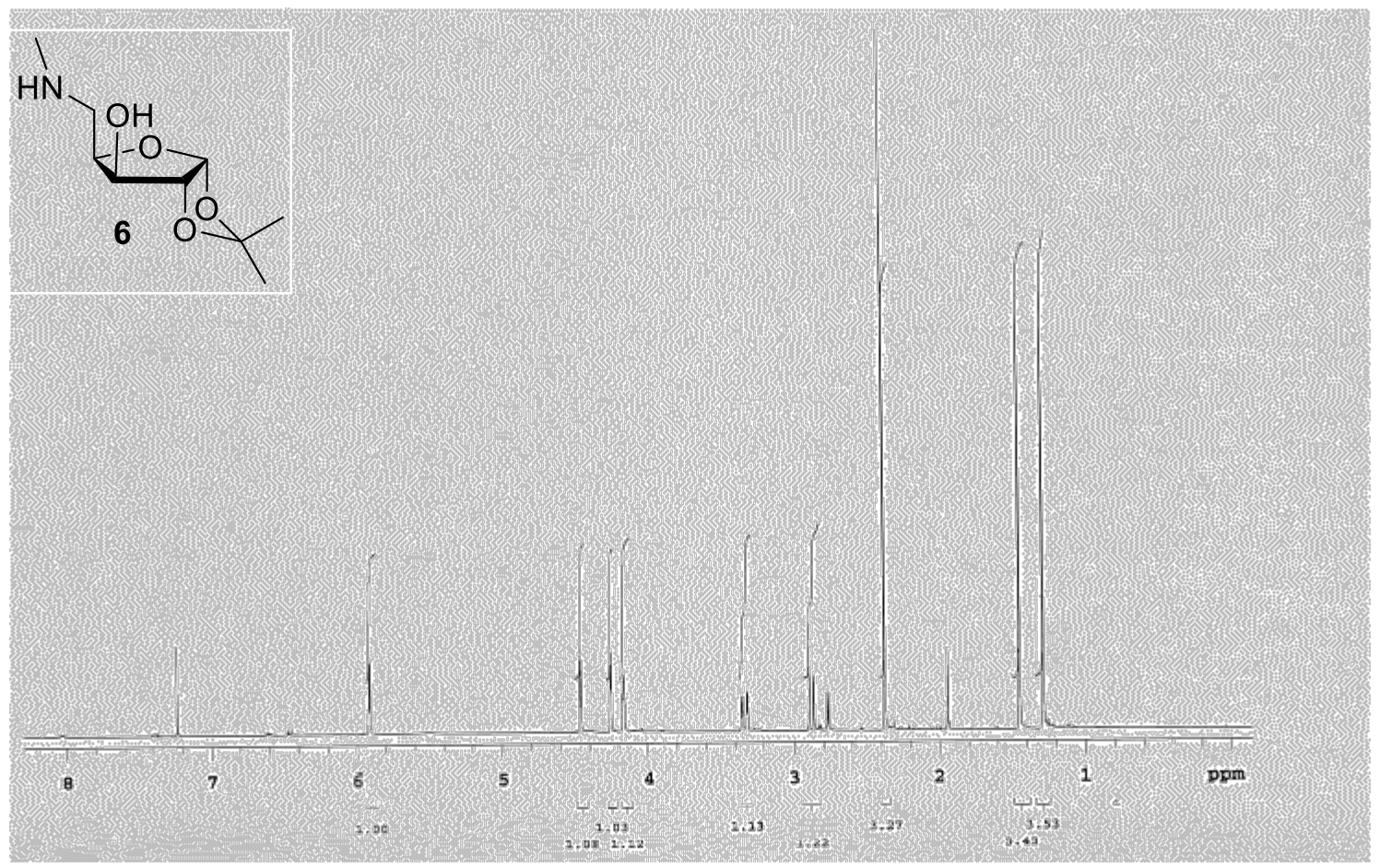

${ }^{1} \mathrm{H} \mathrm{NMR}\left(\mathrm{CDCl}_{3}, 400 \mathrm{MHz}\right)$

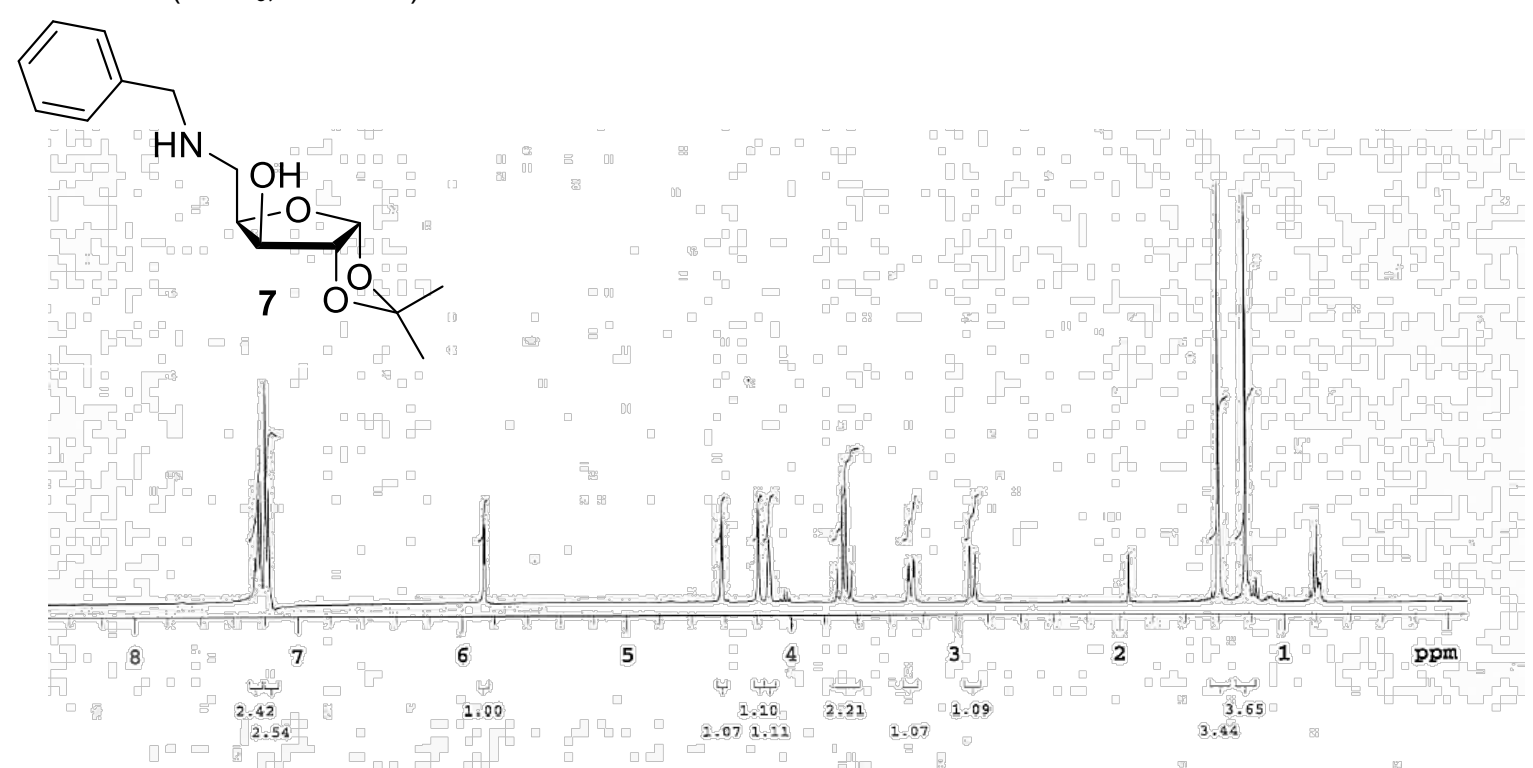


${ }^{1} \mathrm{H} \mathrm{NMR}\left(\mathrm{CDCl}_{3}, 250 \mathrm{MHz}\right)$

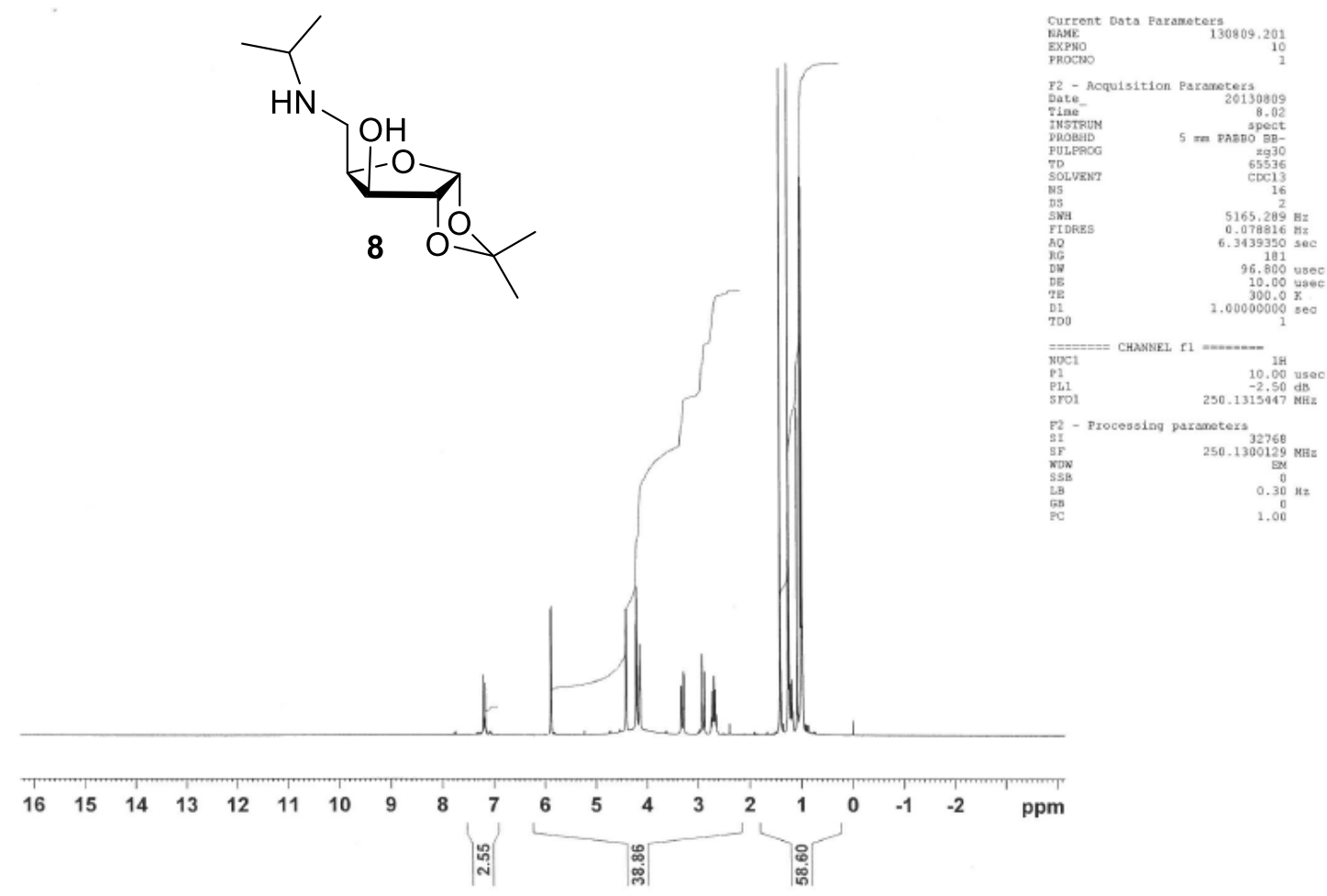

${ }^{13} \mathrm{C}\left\{{ }^{1} \mathrm{H}\right\} \mathrm{NMR}\left(\mathrm{CDCl}_{3}, 63 \mathrm{MHz}\right)$

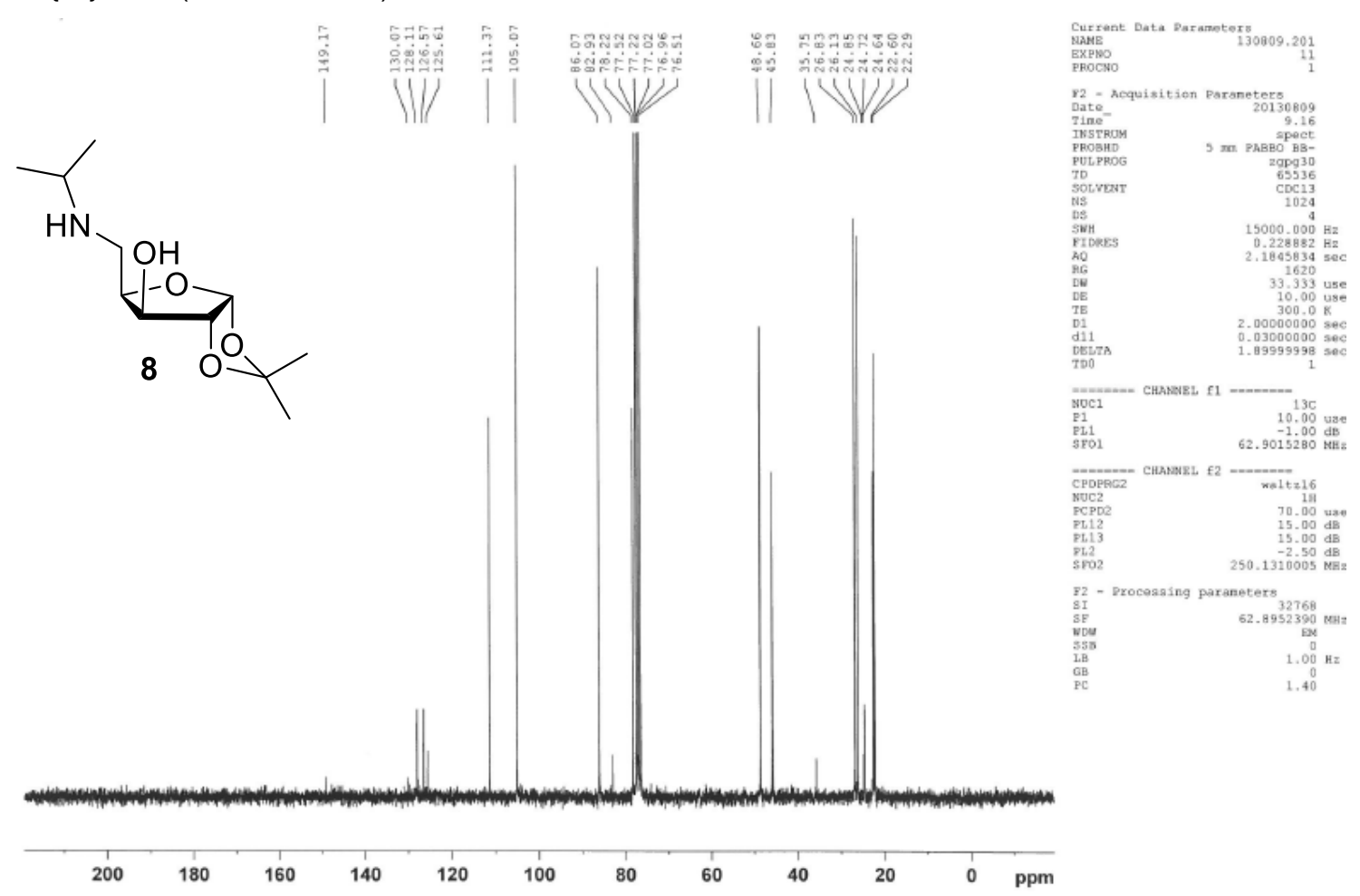


${ }^{1} \mathrm{H} \mathrm{NMR}\left(\mathrm{CDCl}_{3}, 250 \mathrm{MHz}\right)$

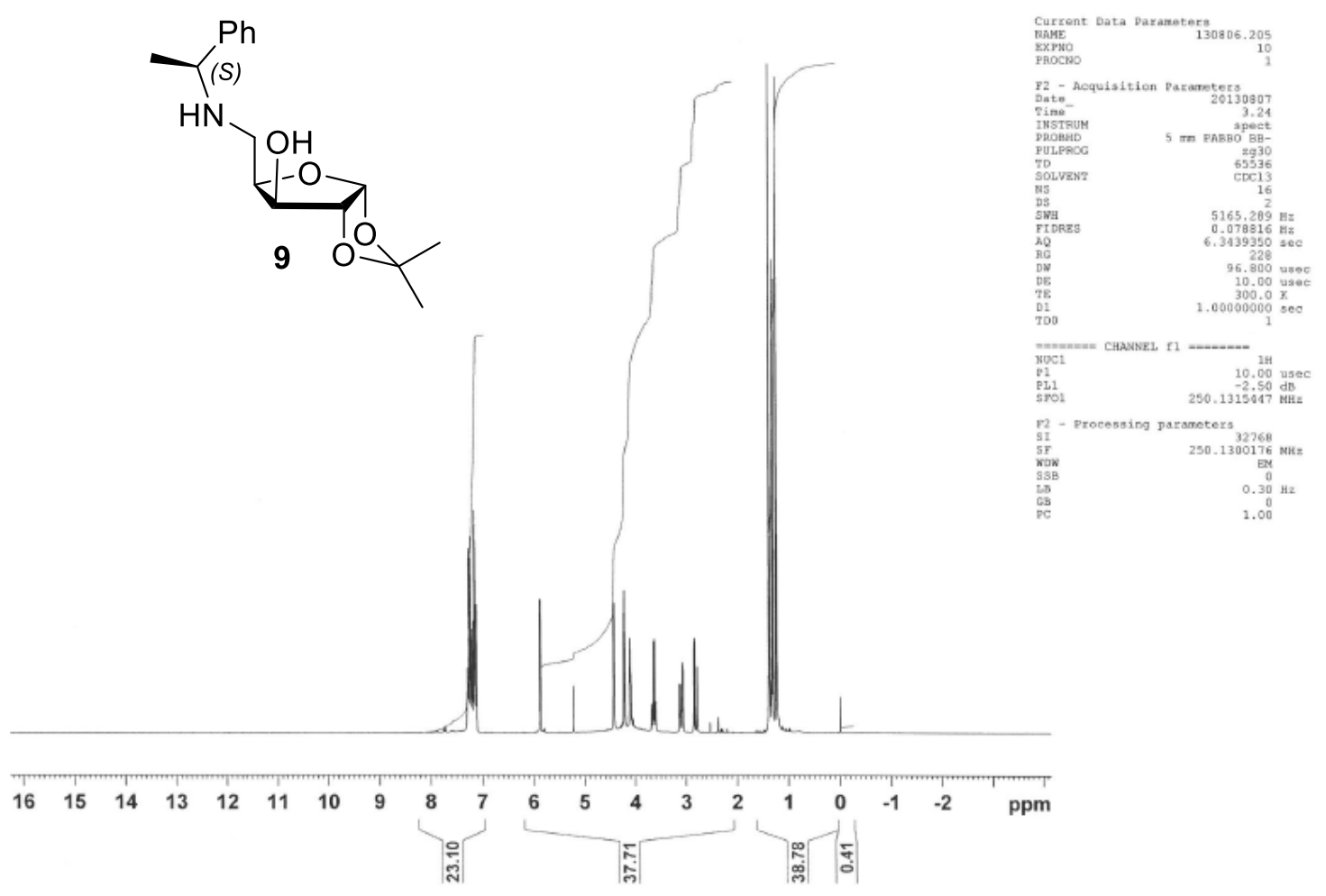

${ }^{13} \mathrm{C}\left\{{ }^{1} \mathrm{H}\right\} \operatorname{NMR}\left(\mathrm{CDCl}_{3}, 63 \mathrm{MHz}\right)$

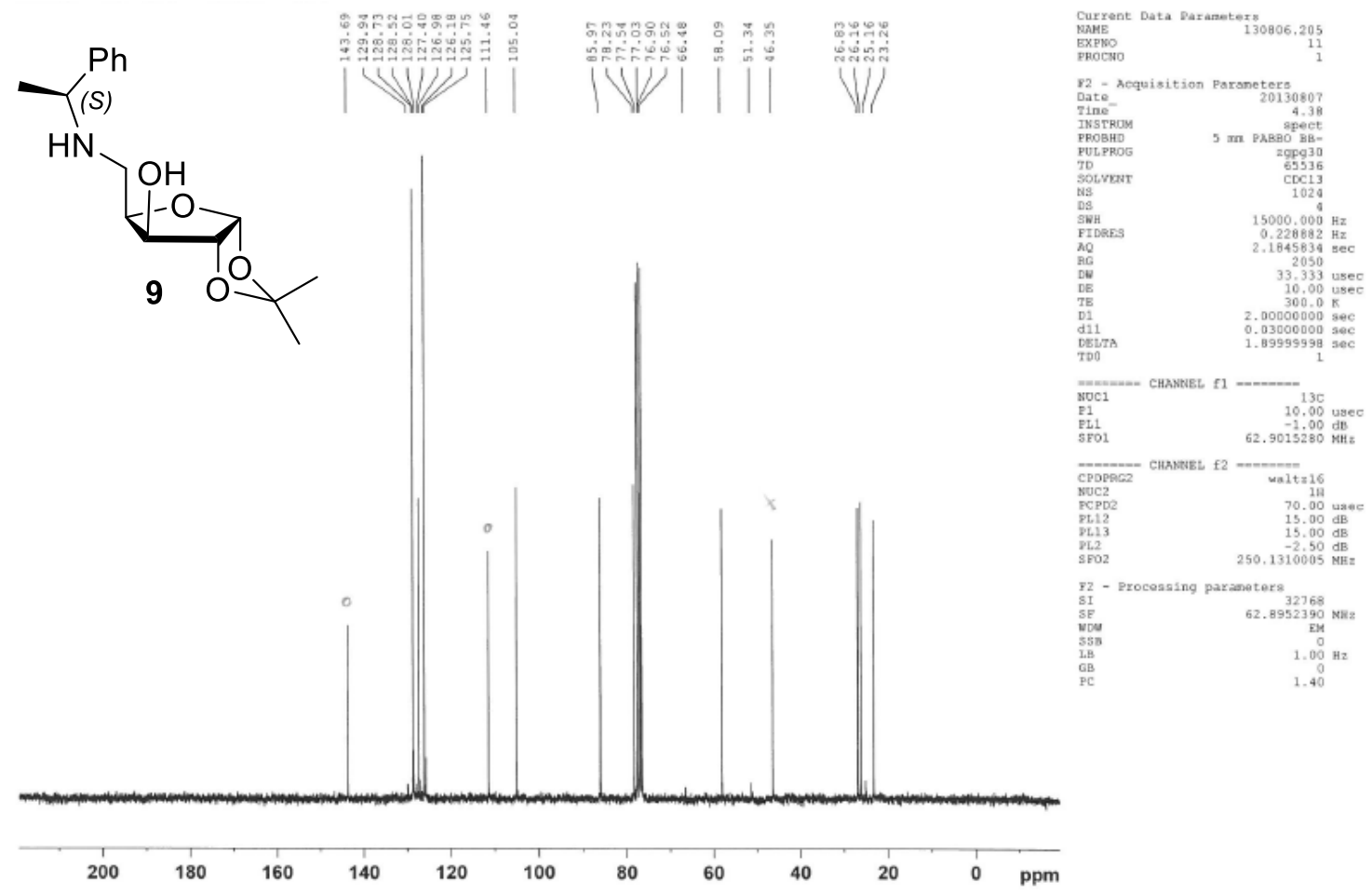


${ }^{1} \mathrm{H} \mathrm{NMR}\left(\mathrm{CDCl}_{3}, 250 \mathrm{MHz}\right)$

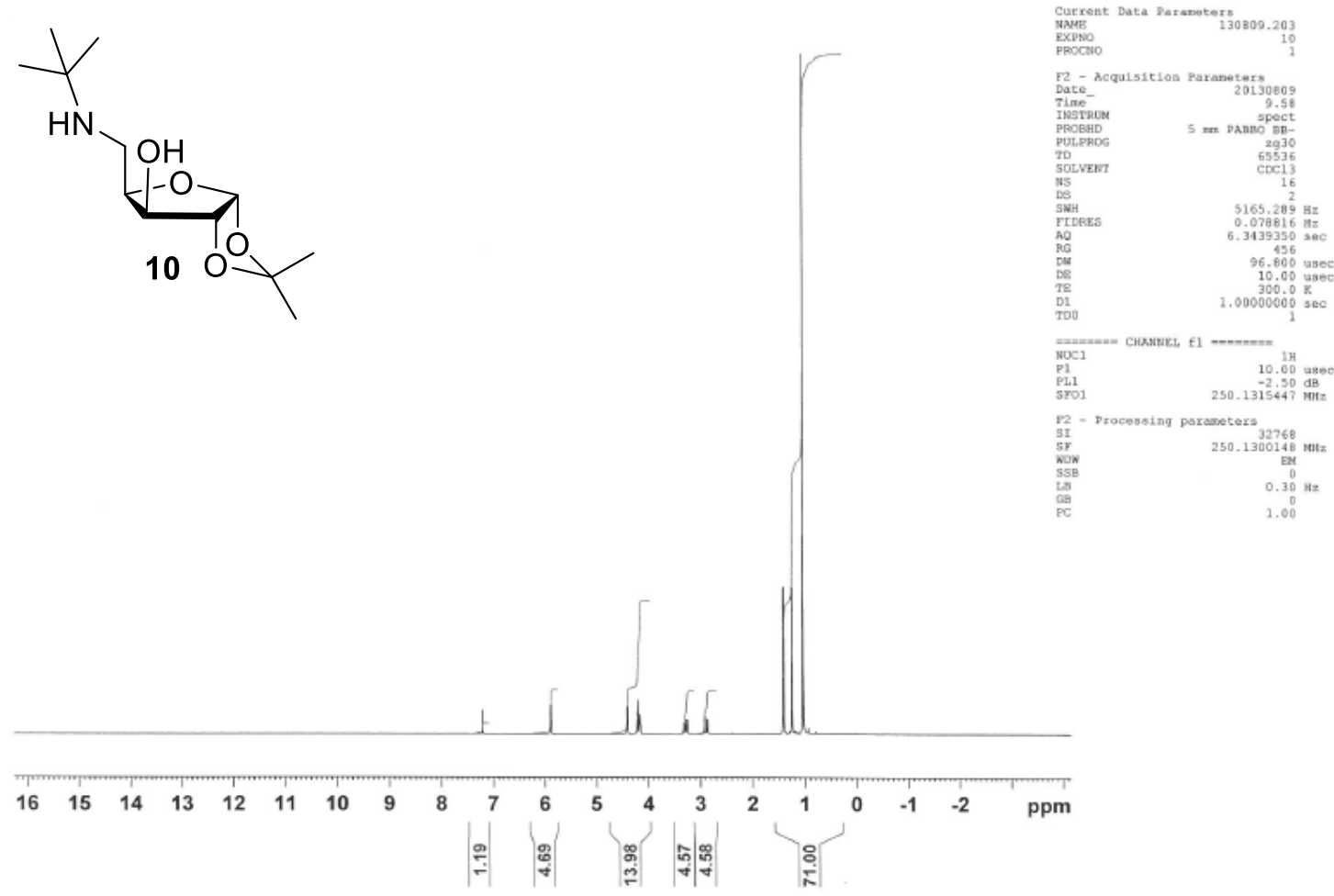

${ }^{13} \mathrm{C}\left\{{ }^{1} \mathrm{H}\right\} \operatorname{NMR}\left(\mathrm{CDCl}_{3}, 63 \mathrm{MHz}\right)$

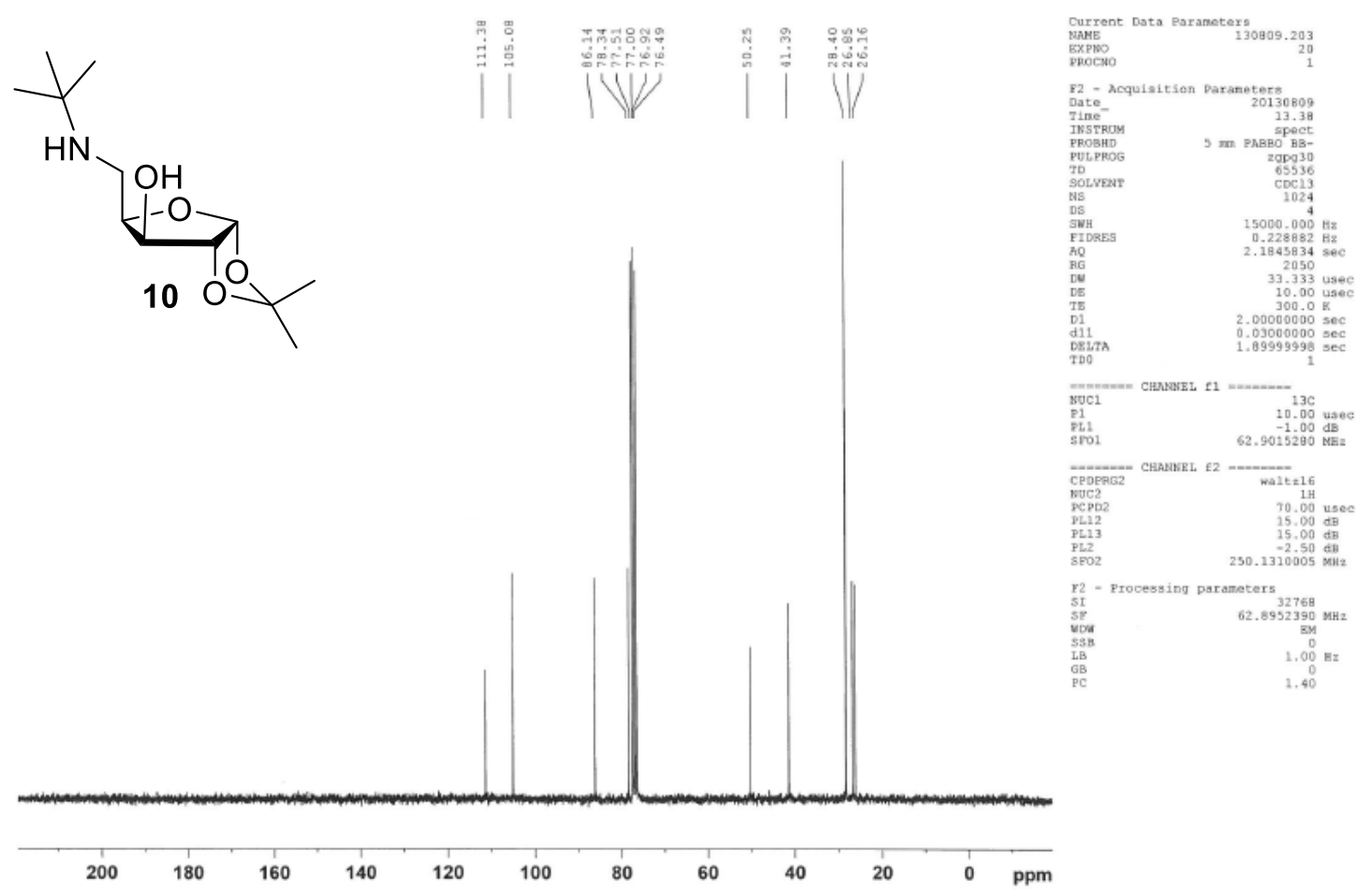




\section{g. Ligands}

${ }^{31} \mathrm{P}\left\{{ }^{1} \mathrm{H}\right\} \mathrm{NMR}\left(\mathrm{C}_{6} \mathrm{D}_{6}, 101 \mathrm{MHz}\right)$

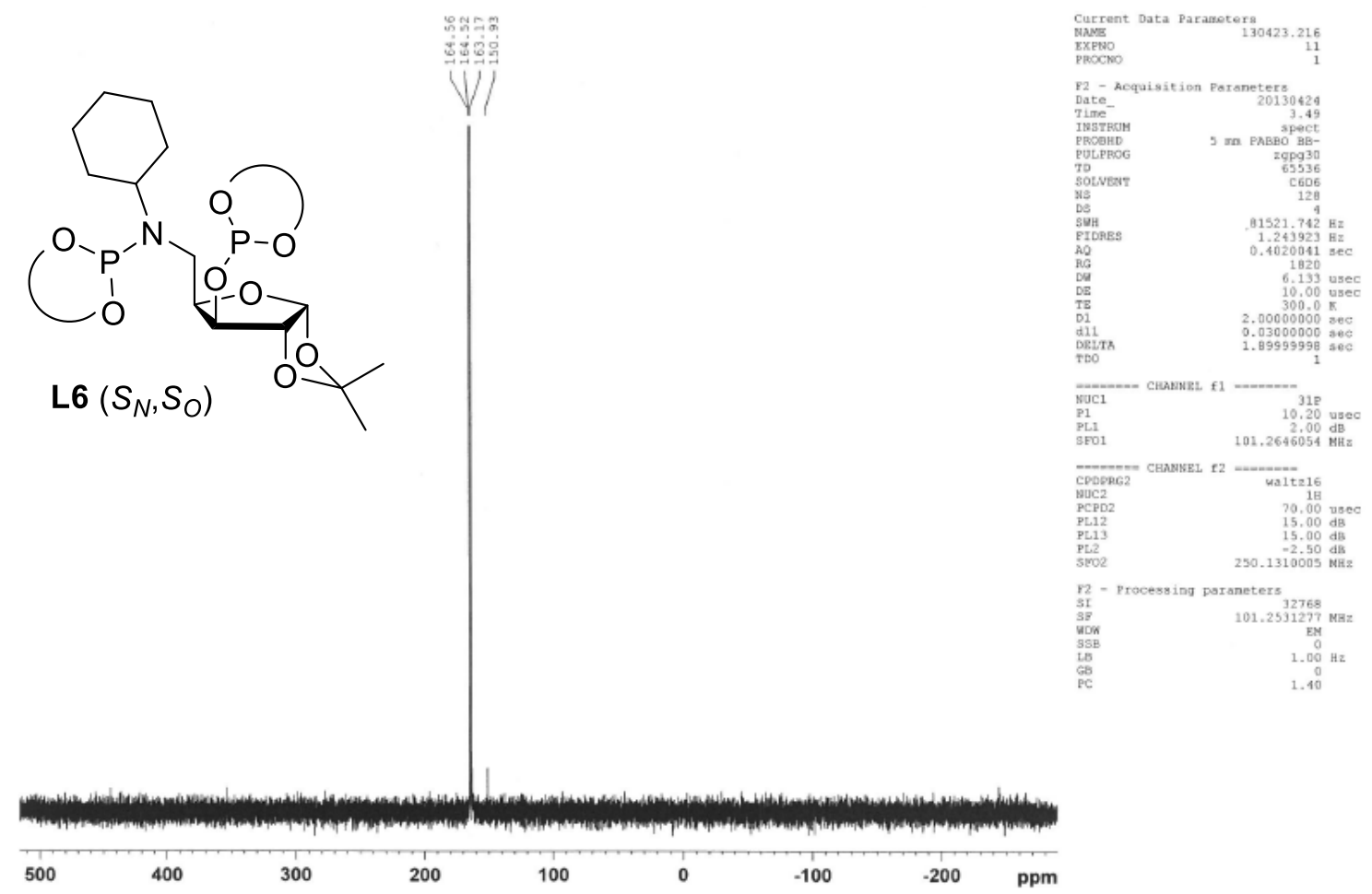

${ }^{1} \mathrm{H} \operatorname{NMR}\left(\mathrm{C}_{6} \mathrm{D}_{6}, 250 \mathrm{MHz}\right)$

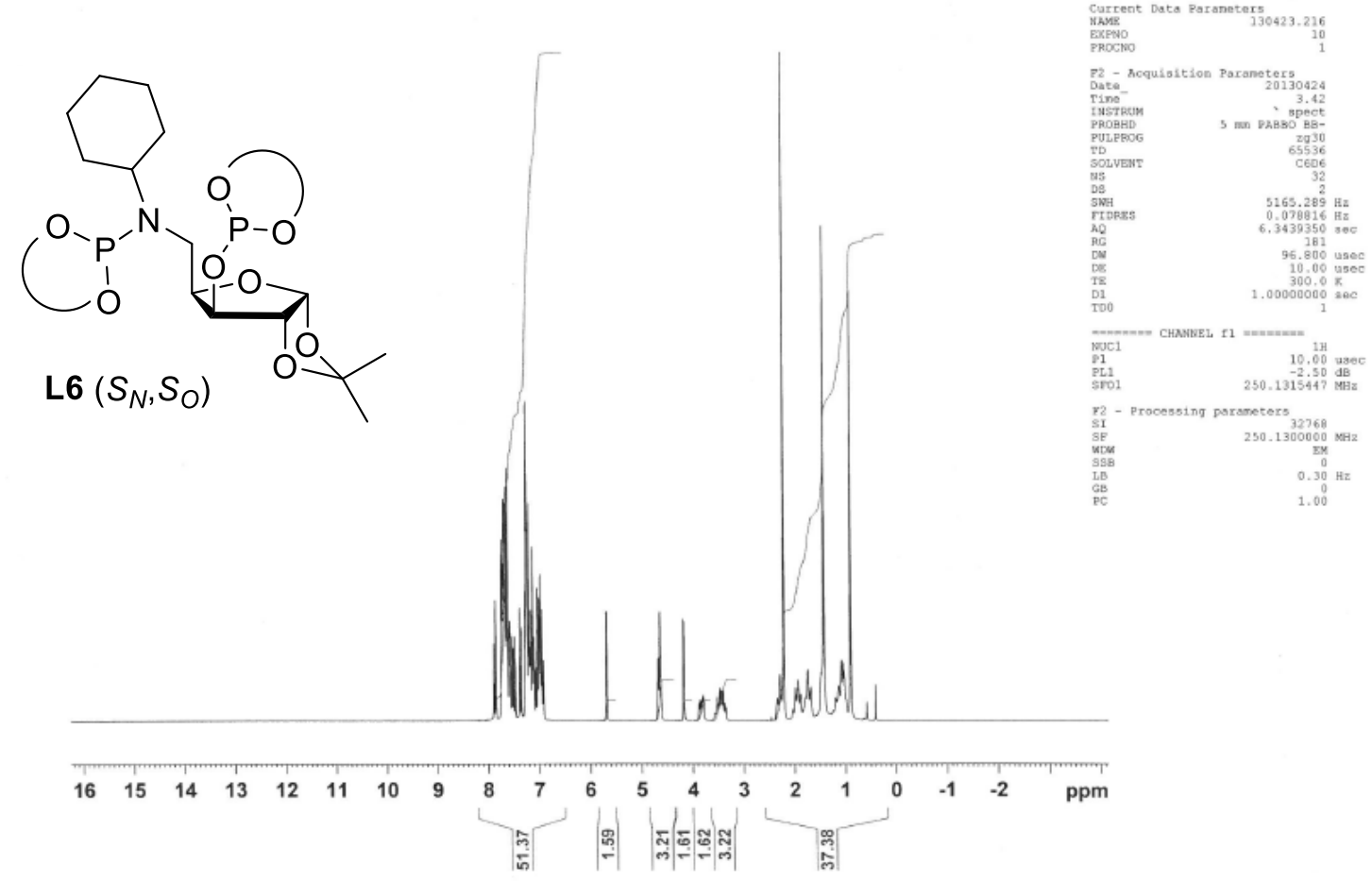


${ }^{31} \mathrm{P}\left\{{ }^{1} \mathrm{H}\right\} \operatorname{NMR}\left(\mathrm{C}_{6} \mathrm{D}_{6}, 101 \mathrm{MHz}\right)$

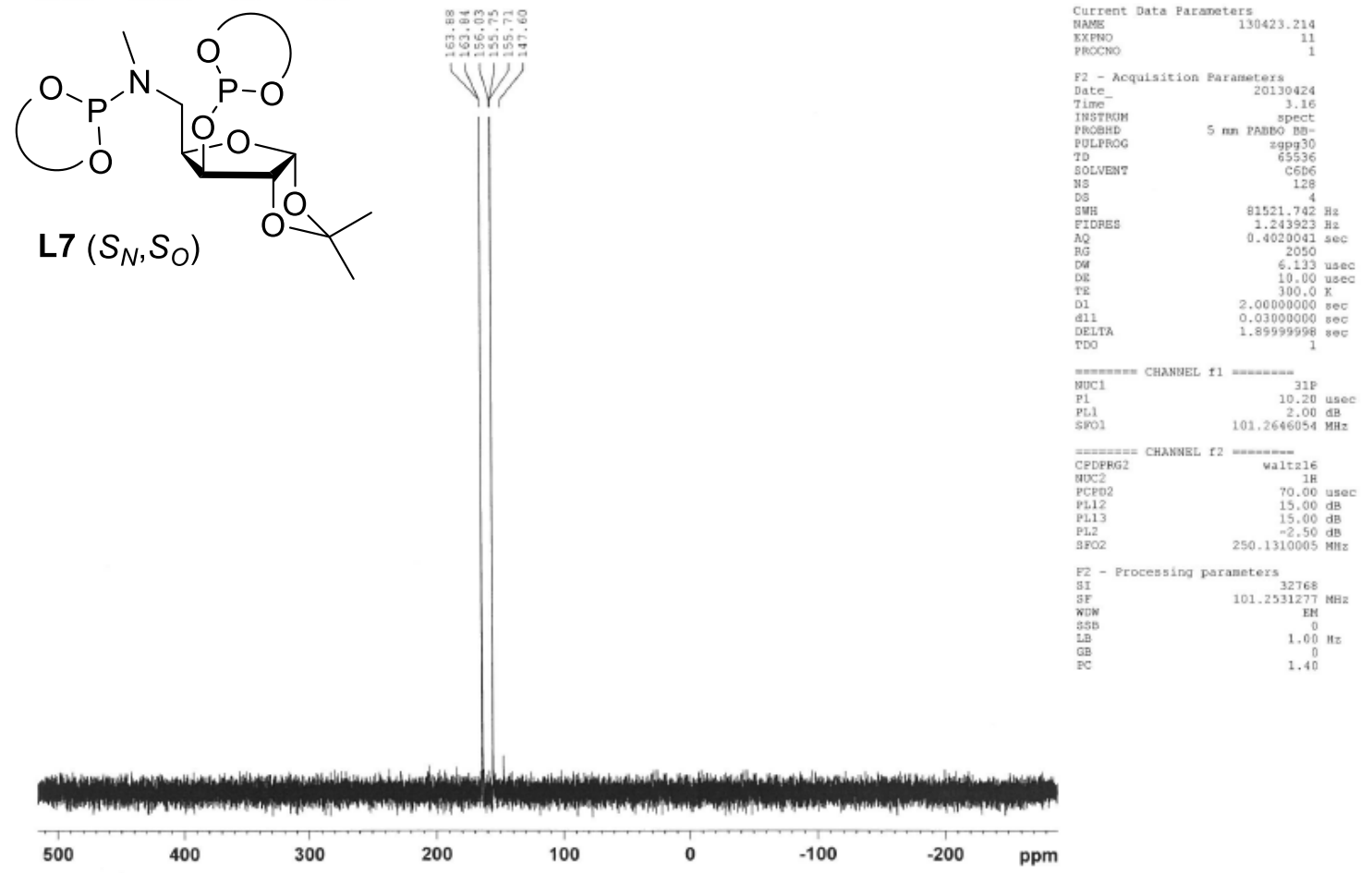

${ }^{1} \mathrm{H}$ NMR $\left(\mathrm{C}_{6} \mathrm{D}_{6}, 250 \mathrm{MHz}\right)$

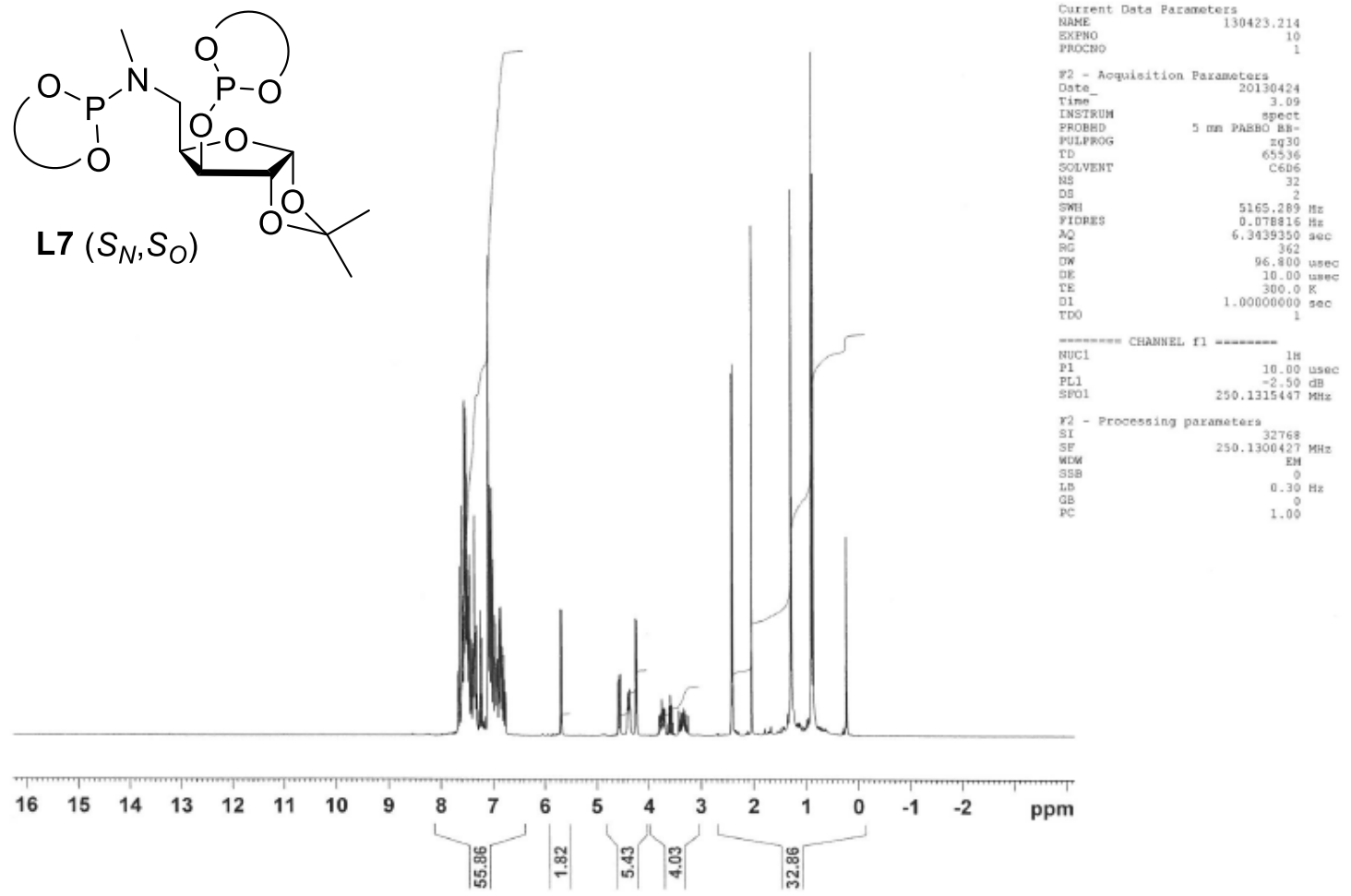


${ }^{31} \mathrm{P}\left\{{ }^{1} \mathrm{H}\right\} \operatorname{NMR}\left(\mathrm{C}_{6} \mathrm{D}_{6}, 101 \mathrm{MHz}\right)$

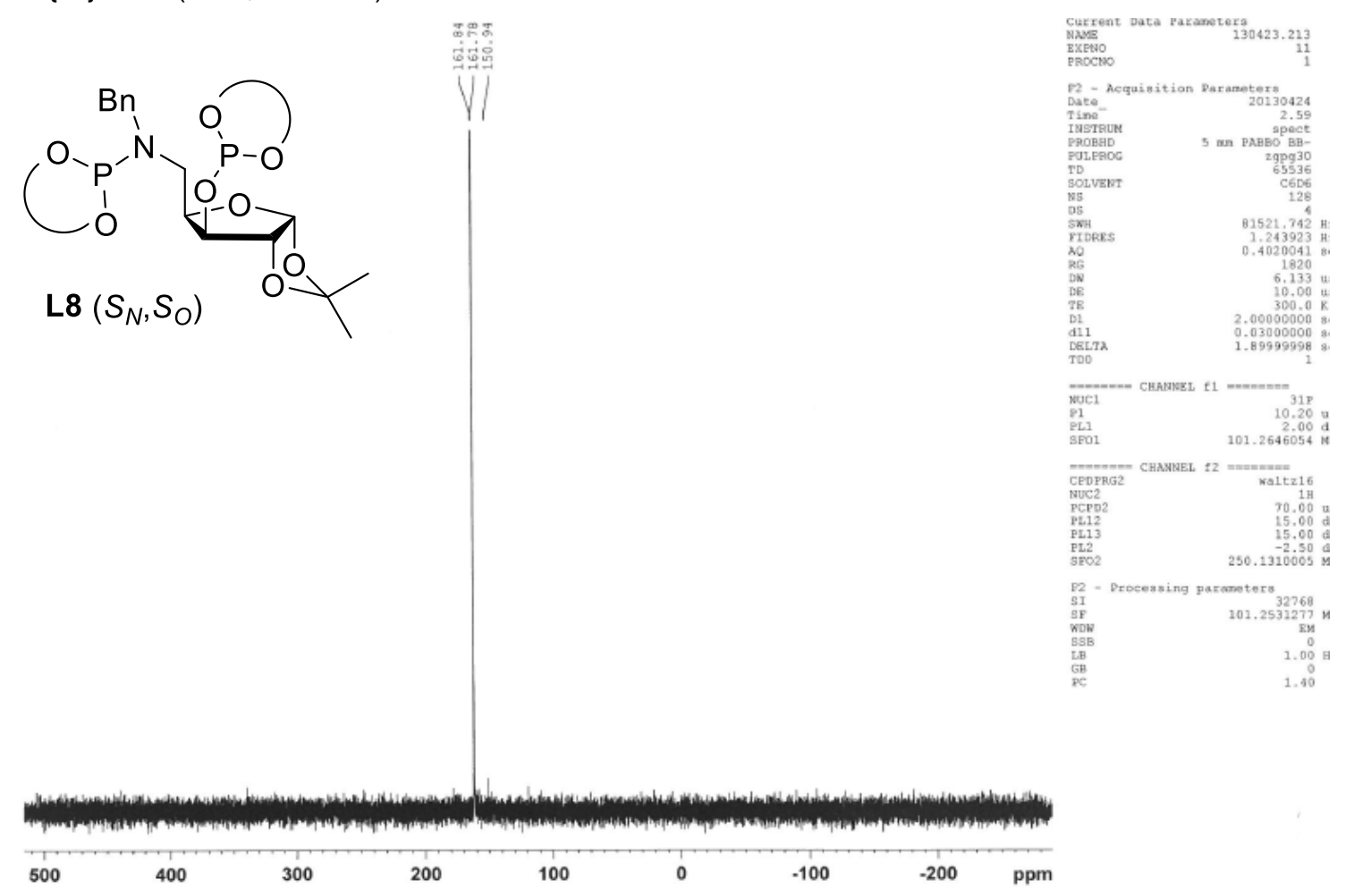

${ }^{1} \mathrm{H}$ NMR $\left(\mathrm{C}_{6} \mathrm{D}_{6}, 250 \mathrm{MHz}\right)$
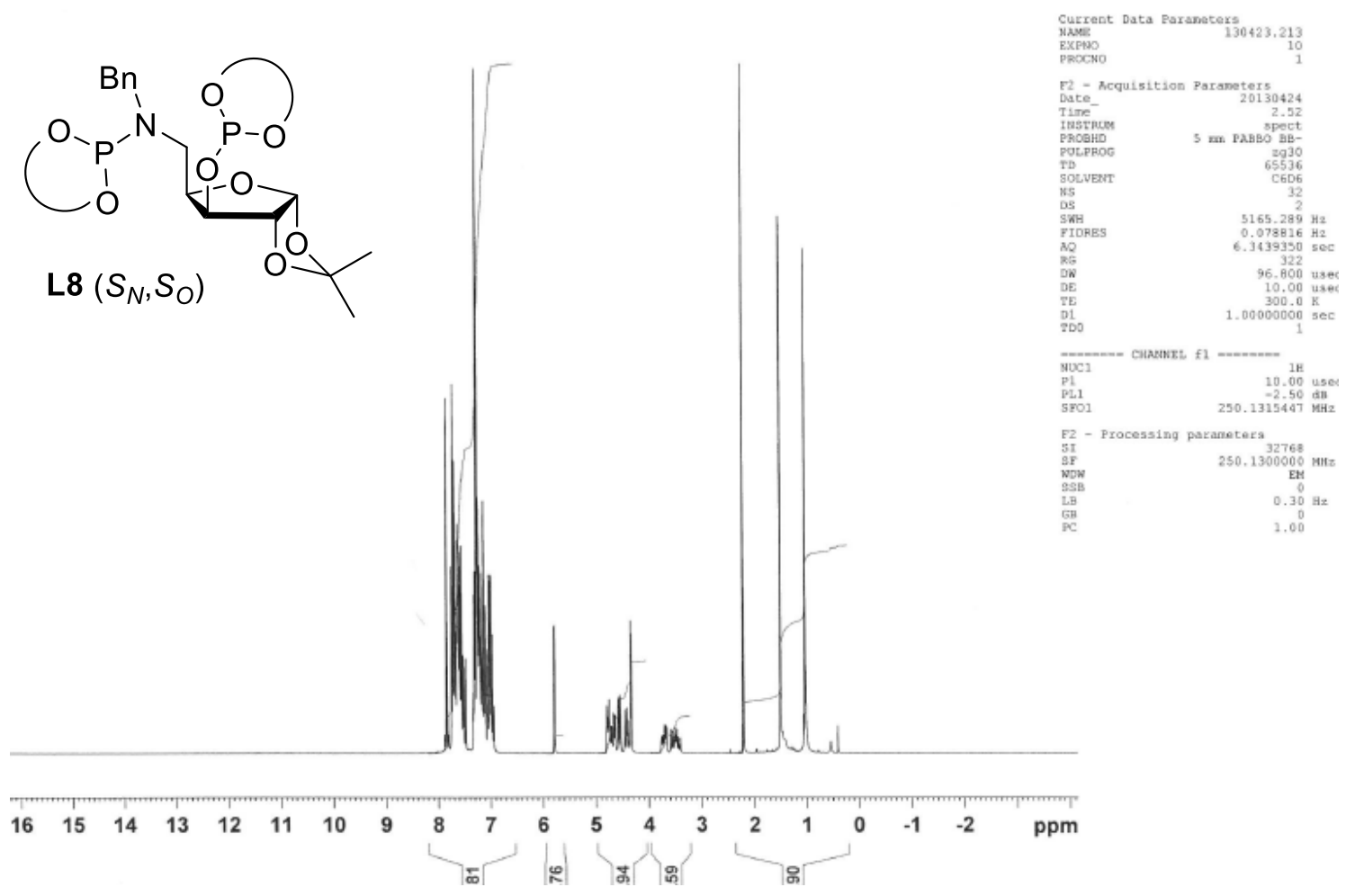
${ }^{31} \mathrm{P}\left\{{ }^{1} \mathrm{H}\right\} \operatorname{NMR}\left(\mathrm{C}_{6} \mathrm{D}_{6}, 101 \mathrm{MHz}\right)$

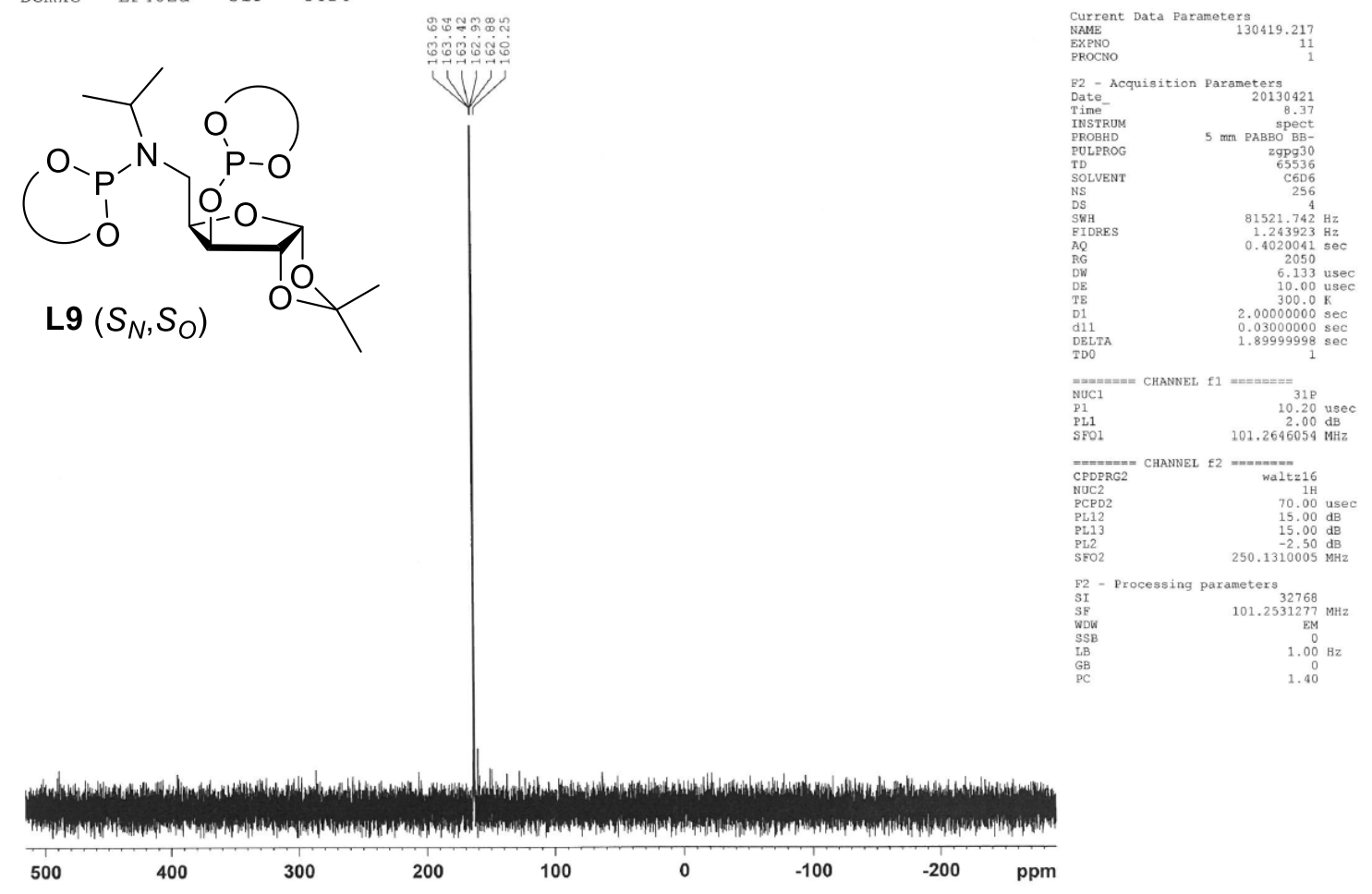

${ }^{1} \mathrm{H}$ NMR $\left(\mathrm{C}_{6} \mathrm{D}_{6}, 250 \mathrm{MHz}\right)$

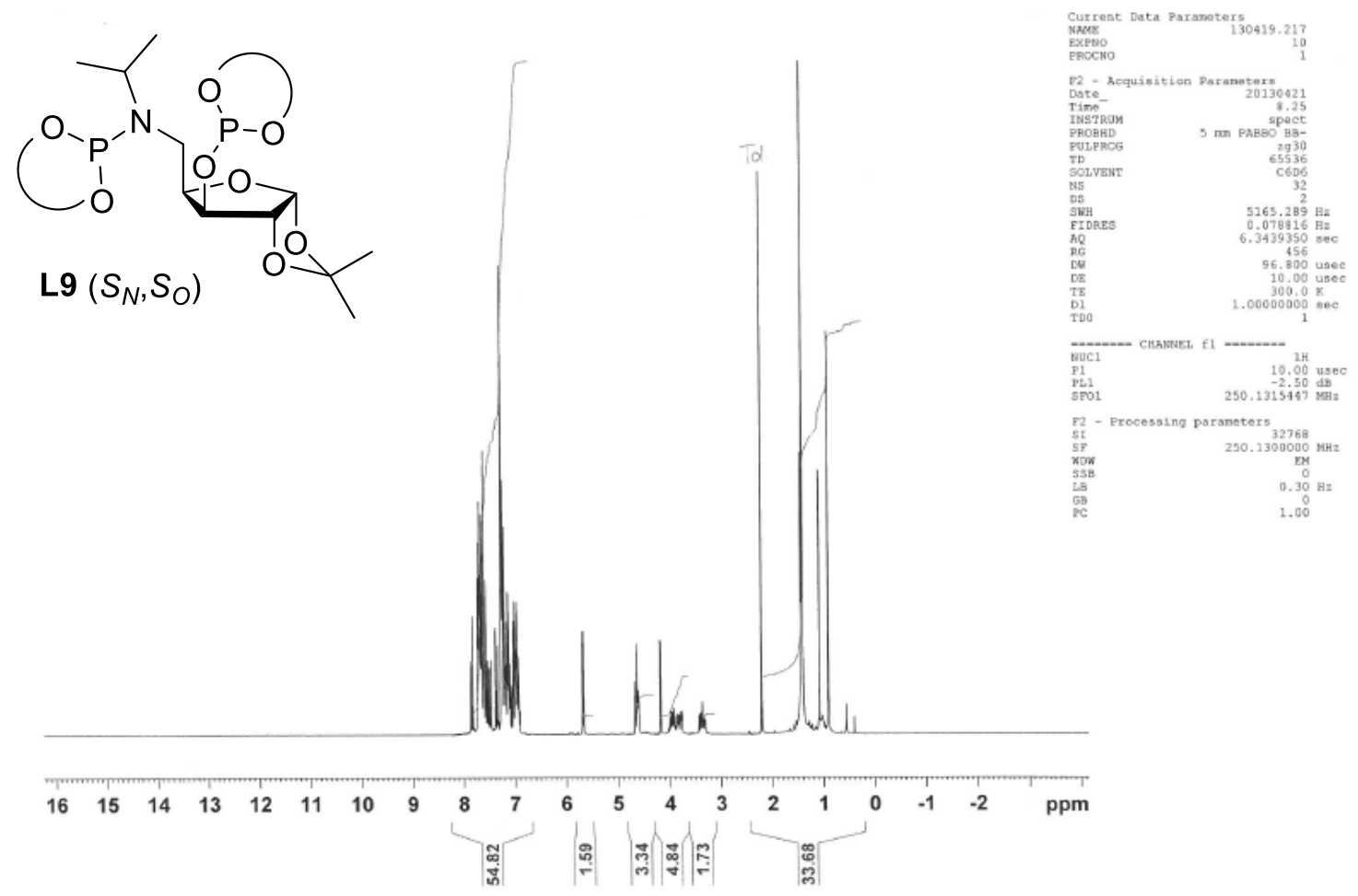


${ }^{31} \mathrm{P}\left\{{ }^{1} \mathrm{H}\right\} \operatorname{NMR}\left(\mathrm{C}_{6} \mathrm{D}_{6}, 121 \mathrm{MHz}\right)$

Domke LP398a 31P C6D6

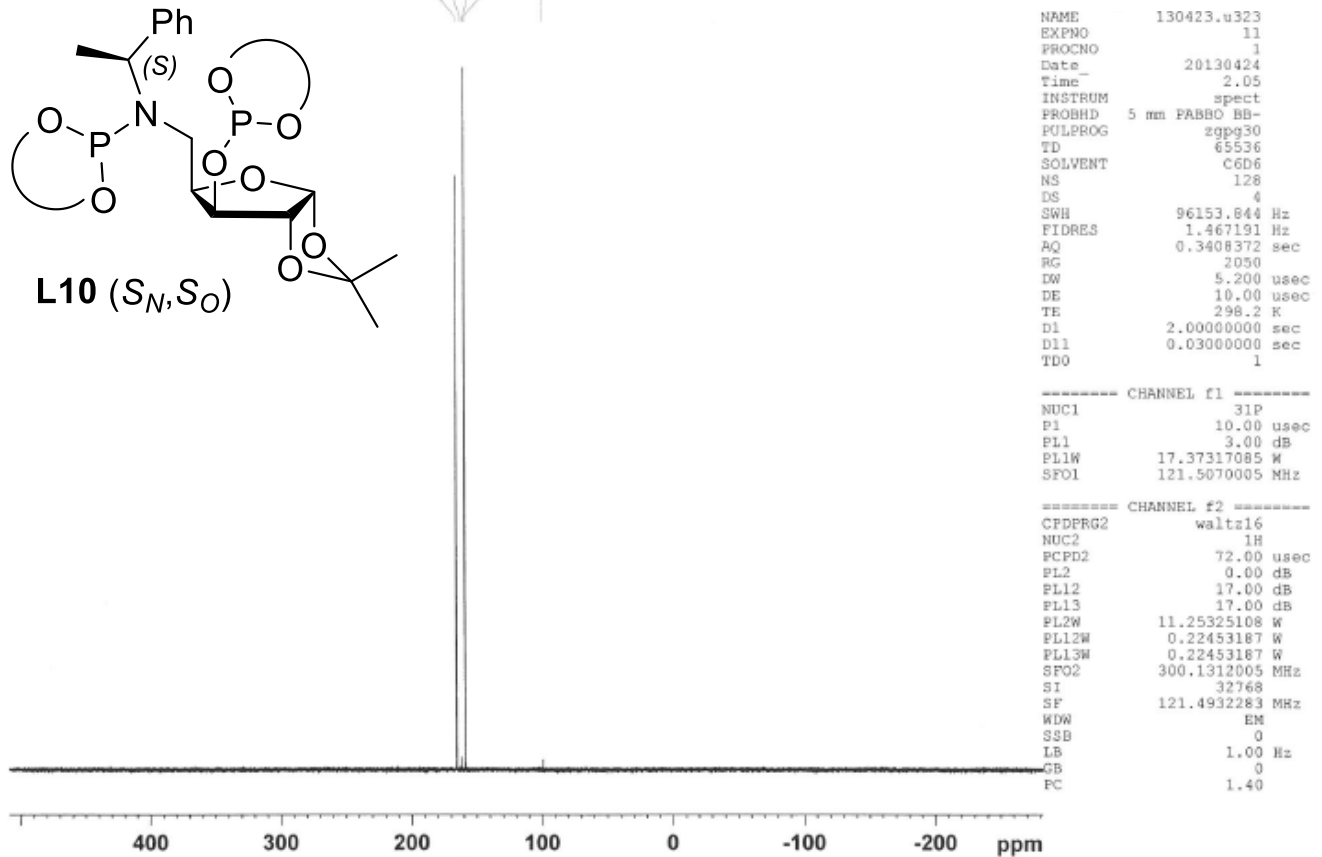

${ }^{1} \mathrm{H} \mathrm{NMR}\left(\mathrm{C}_{6} \mathrm{D}_{6}, 300 \mathrm{MHz}\right)$
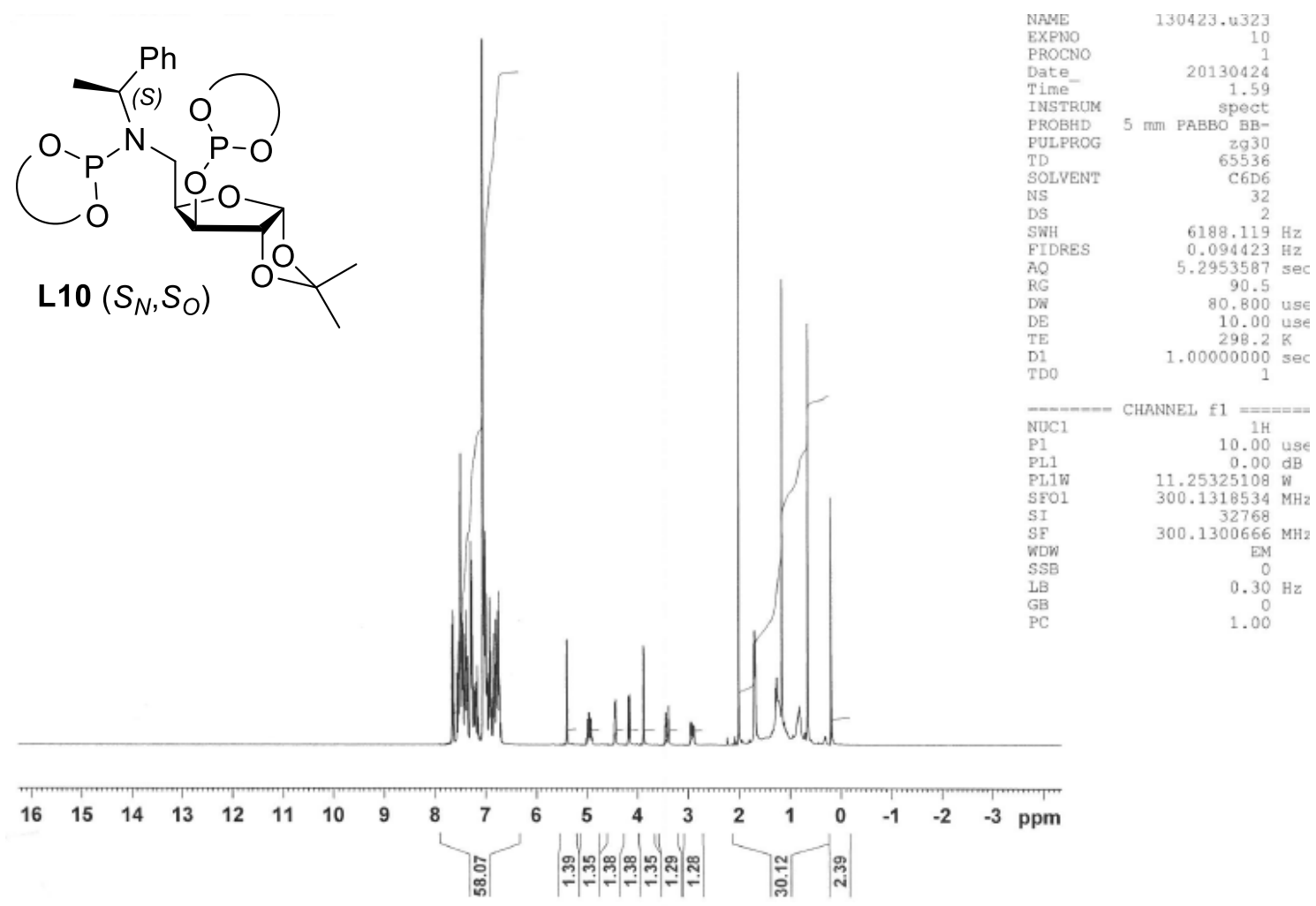
${ }^{31} \mathrm{P}\left\{{ }^{1} \mathrm{H}\right\} \operatorname{NMR}\left(\mathrm{C}_{6} \mathrm{D}_{6}, 101 \mathrm{MHz}\right)$
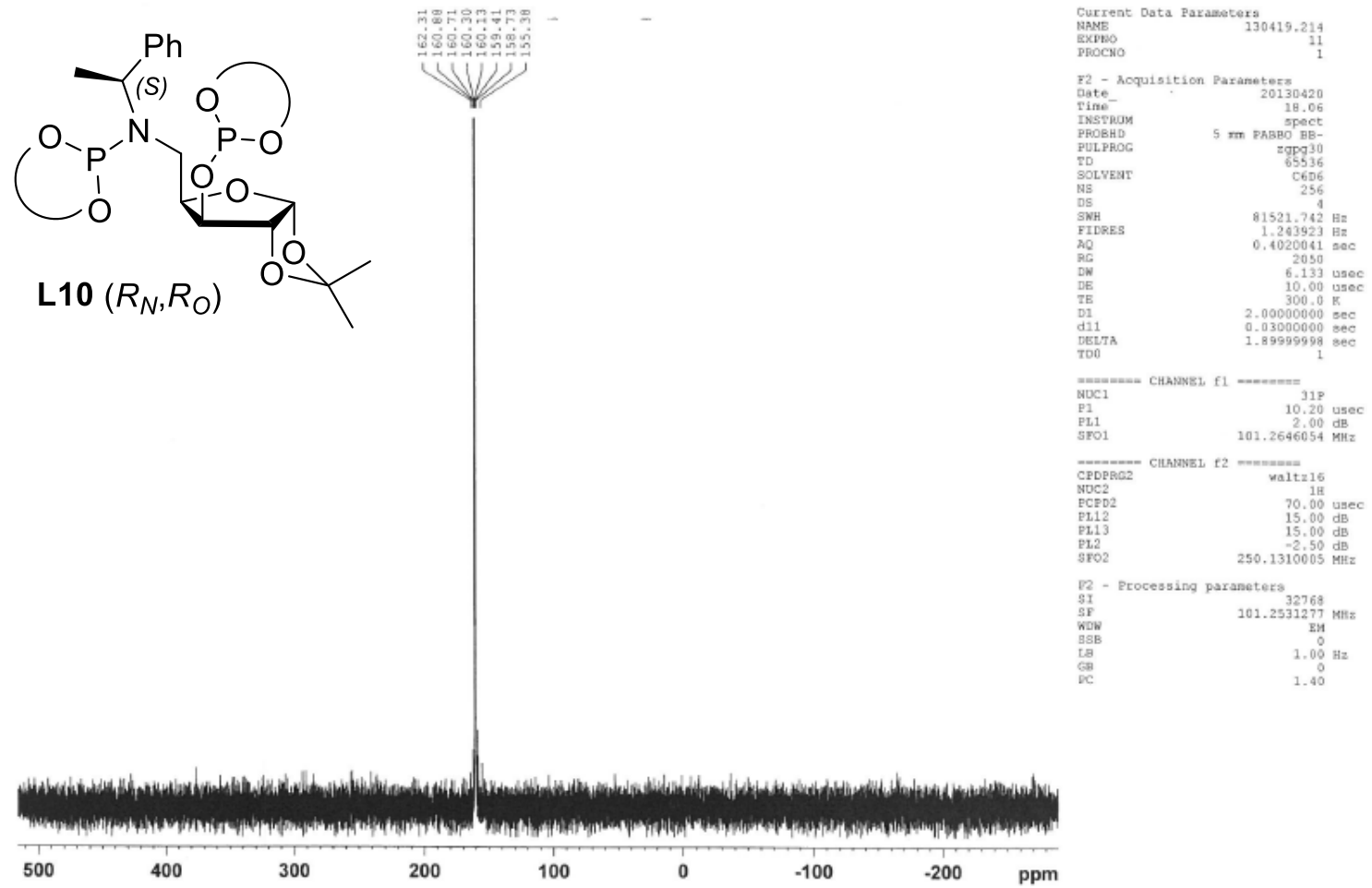

${ }^{1} \mathrm{H} \operatorname{NMR}\left(\mathrm{C}_{6} \mathrm{D}_{6}, 250 \mathrm{MHz}\right)$
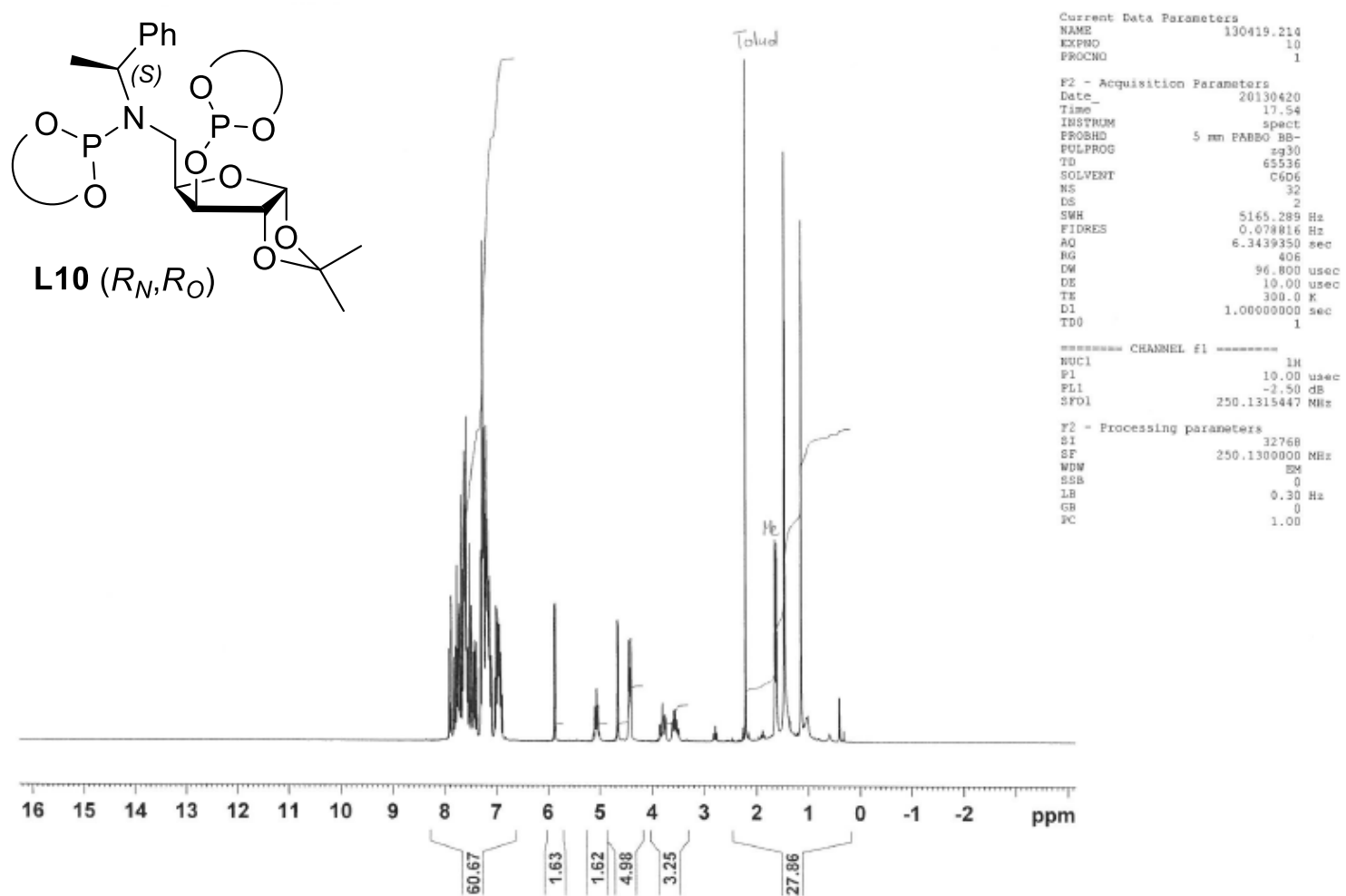
${ }^{31} \mathrm{P}\left\{{ }^{1} \mathrm{H}\right\} \mathrm{NMR}\left(\mathrm{CDCl}_{3}, 101 \mathrm{MHz}\right)$

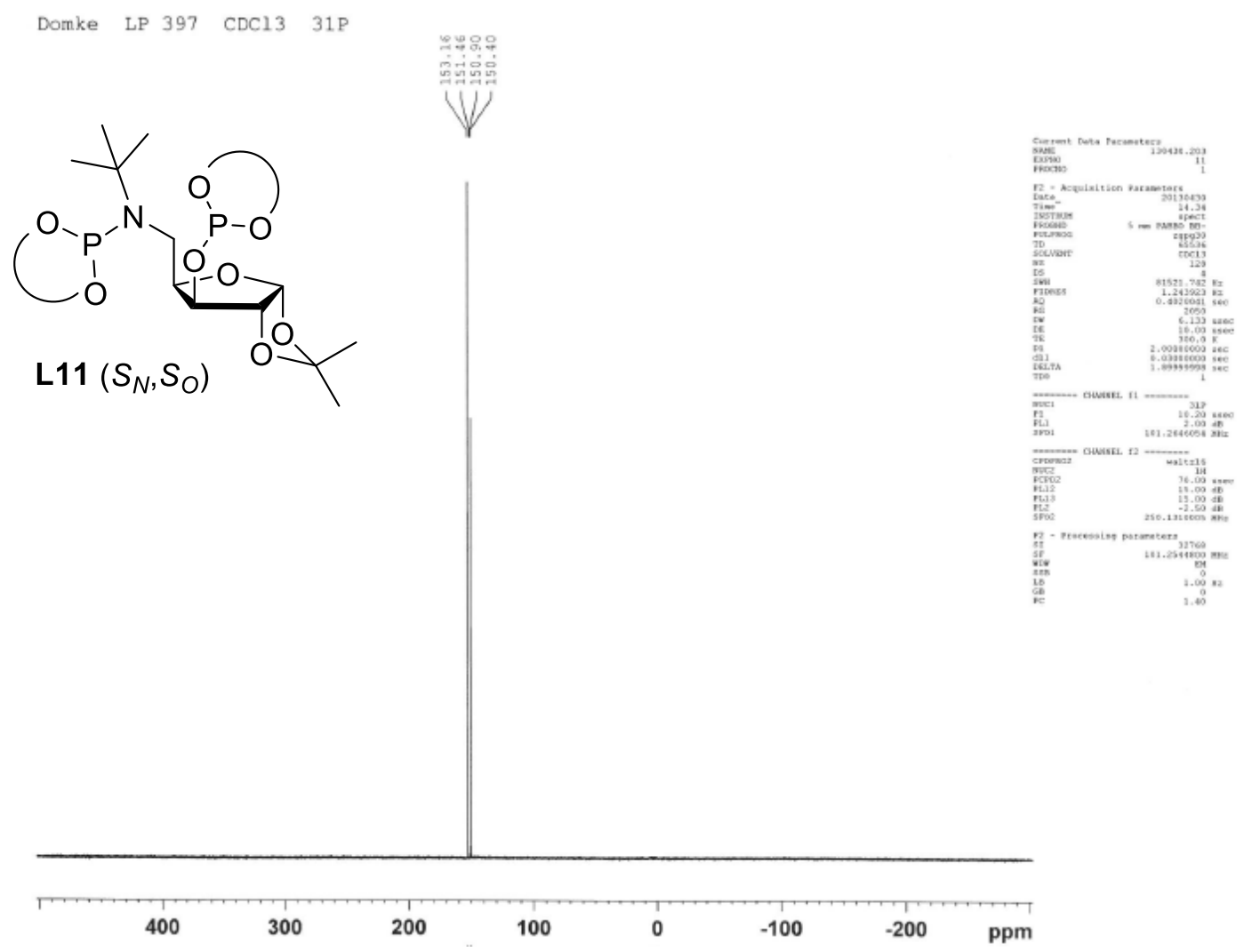

${ }^{1} \mathrm{H} \mathrm{NMR}\left(\mathrm{CDCl}_{3}, 250 \mathrm{MHz}\right)$

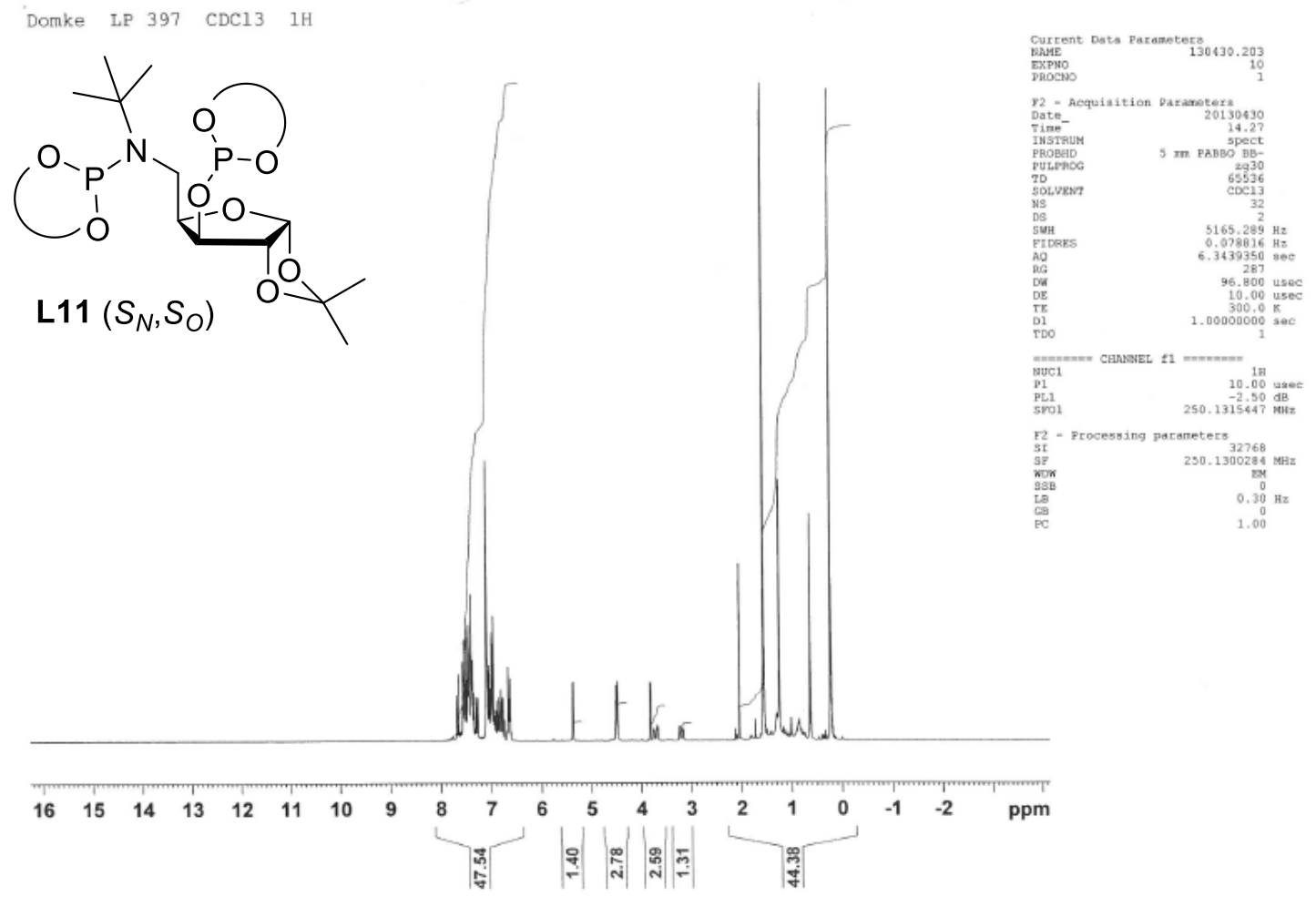


${ }^{31} \mathrm{P}\left\{{ }^{1} \mathrm{H}\right\} \operatorname{NMR}\left(\mathrm{C}_{6} \mathrm{D}_{6}, 121 \mathrm{MHz}\right)$

$31 \mathrm{P}$ in $\mathrm{C6} 6 \mathrm{D}$, Reference $\mathrm{H3PO4}=0.0 \mathrm{ppm}$
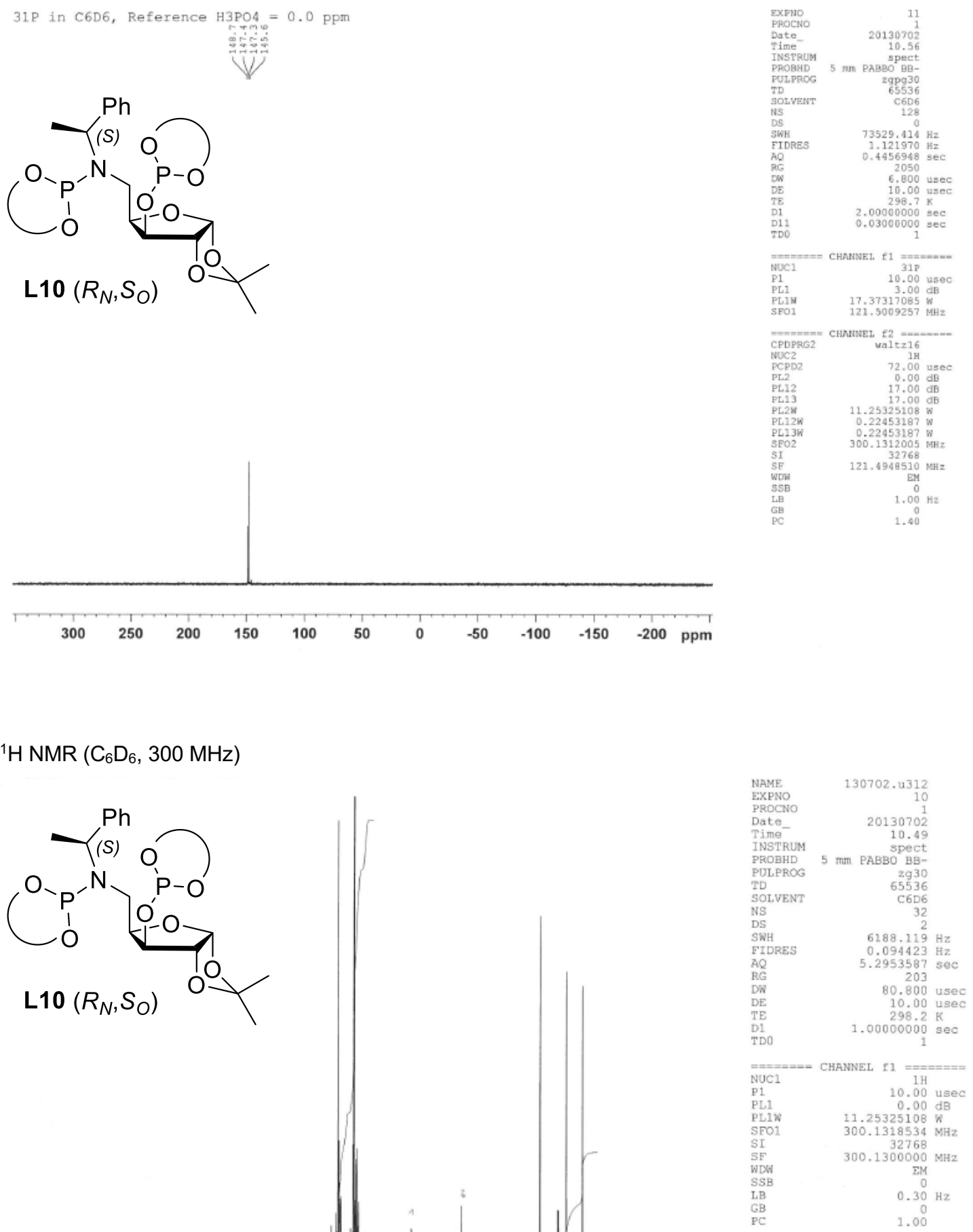

${ }^{1} \mathrm{H}$ NMR $\left(\mathrm{C}_{6} \mathrm{D}_{6}, 300 \mathrm{MHz}\right)$

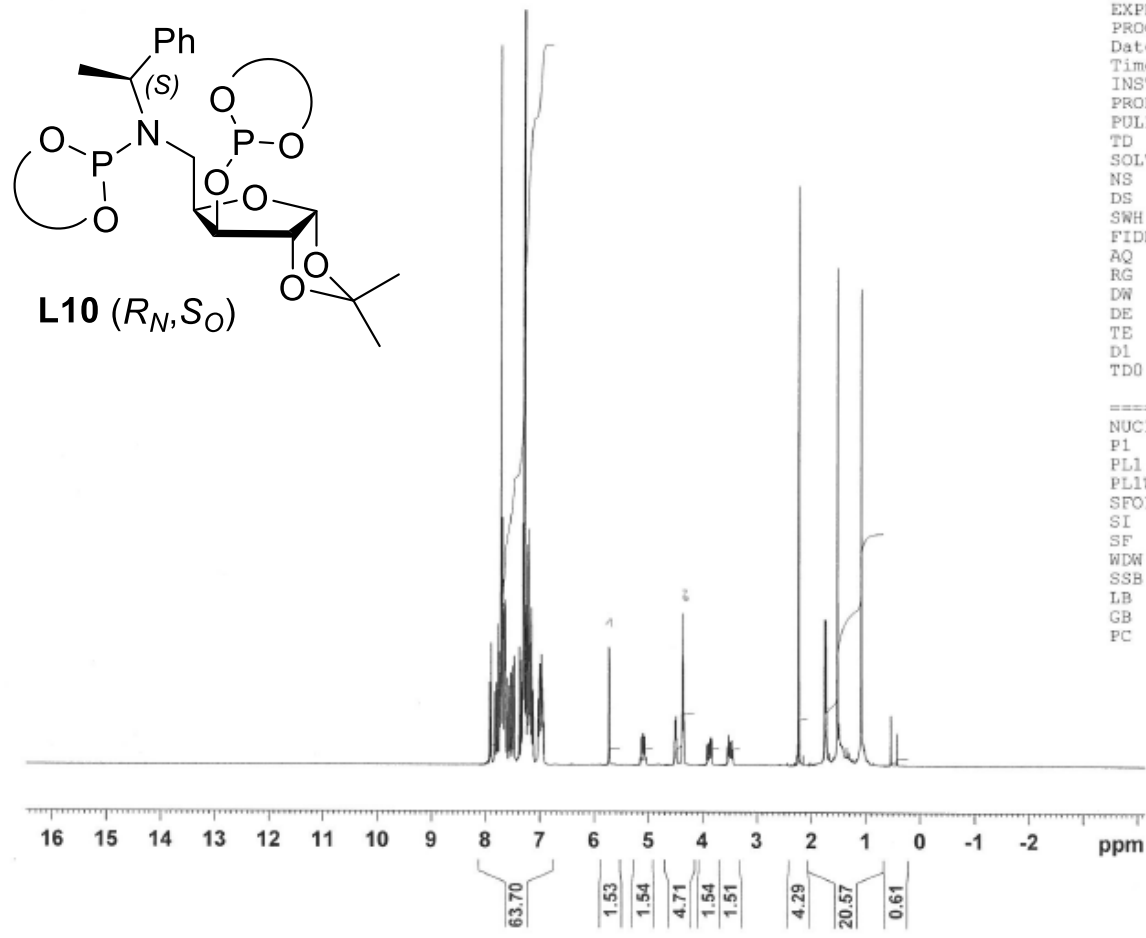


${ }^{31} \mathrm{P}\left\{{ }^{1} \mathrm{H}\right\} \operatorname{NMR}\left(\mathrm{C}_{6} \mathrm{D}_{6}, 121 \mathrm{MHz}\right)$

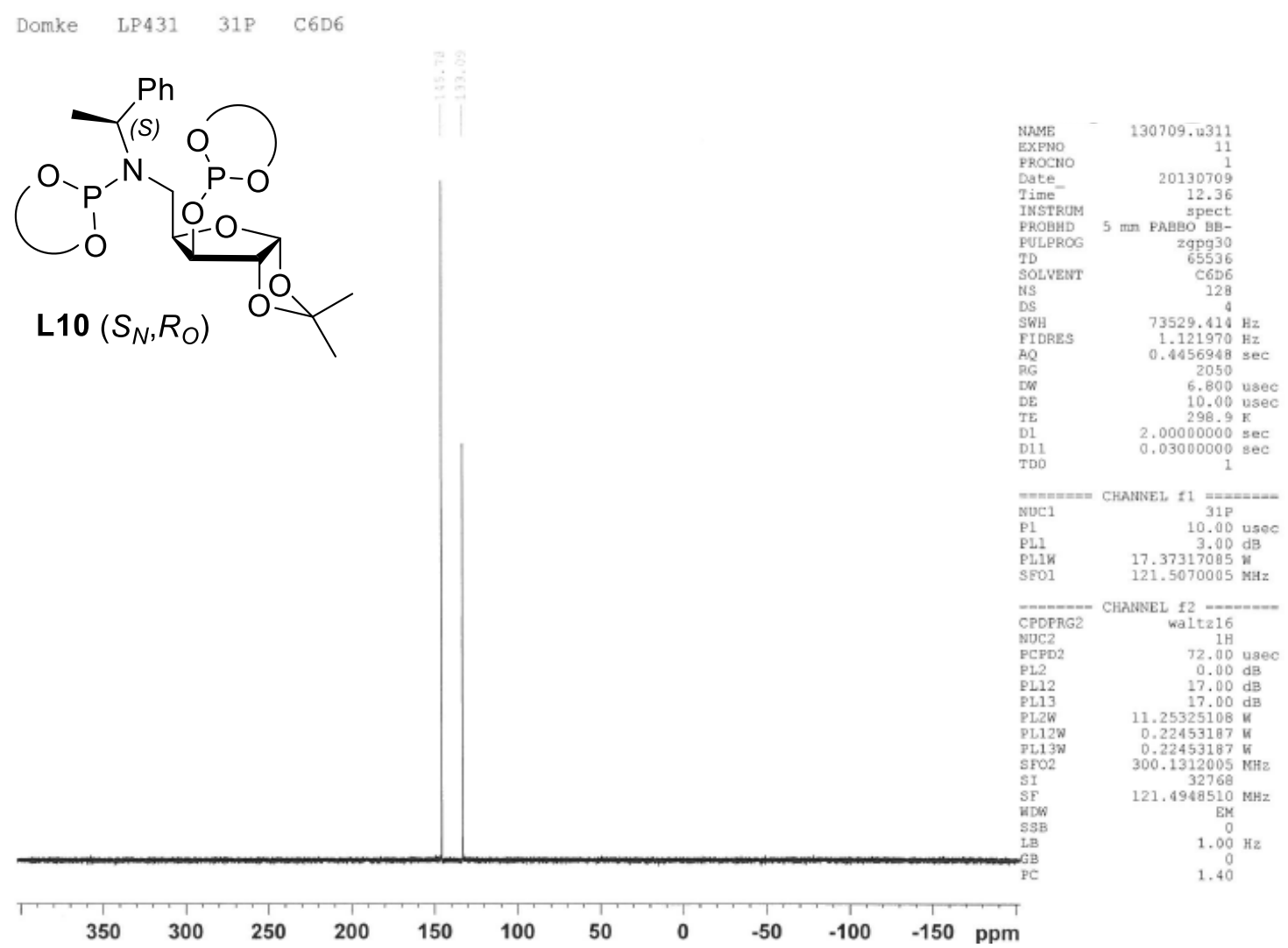

${ }^{1} \mathrm{H} \operatorname{NMR}\left(\mathrm{C}_{6} \mathrm{D}_{6}, 300 \mathrm{MHz}\right)$
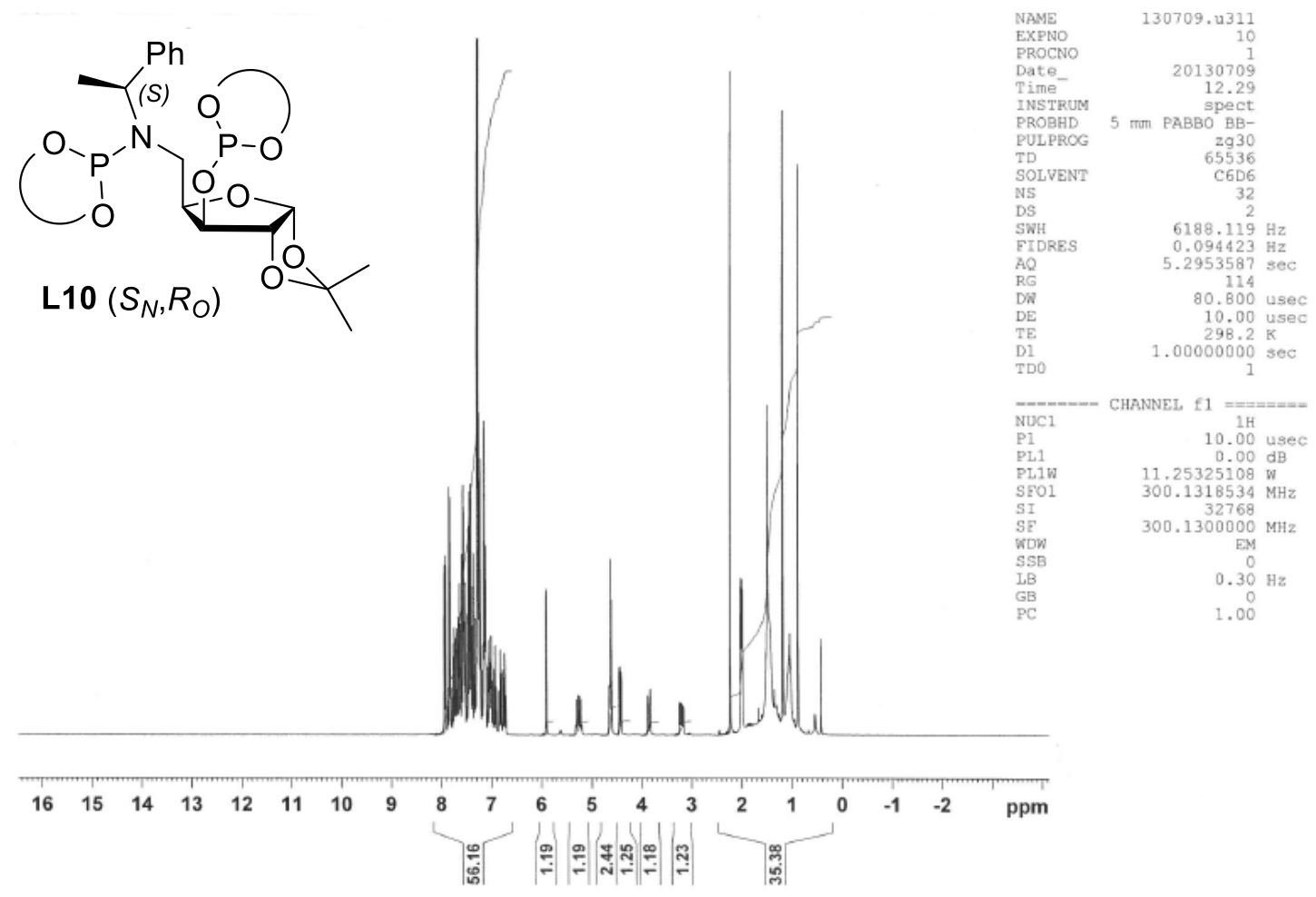
SXIX. GC and HPLC chromatograms traces

\section{a. GC and HPLC alcohols traces}
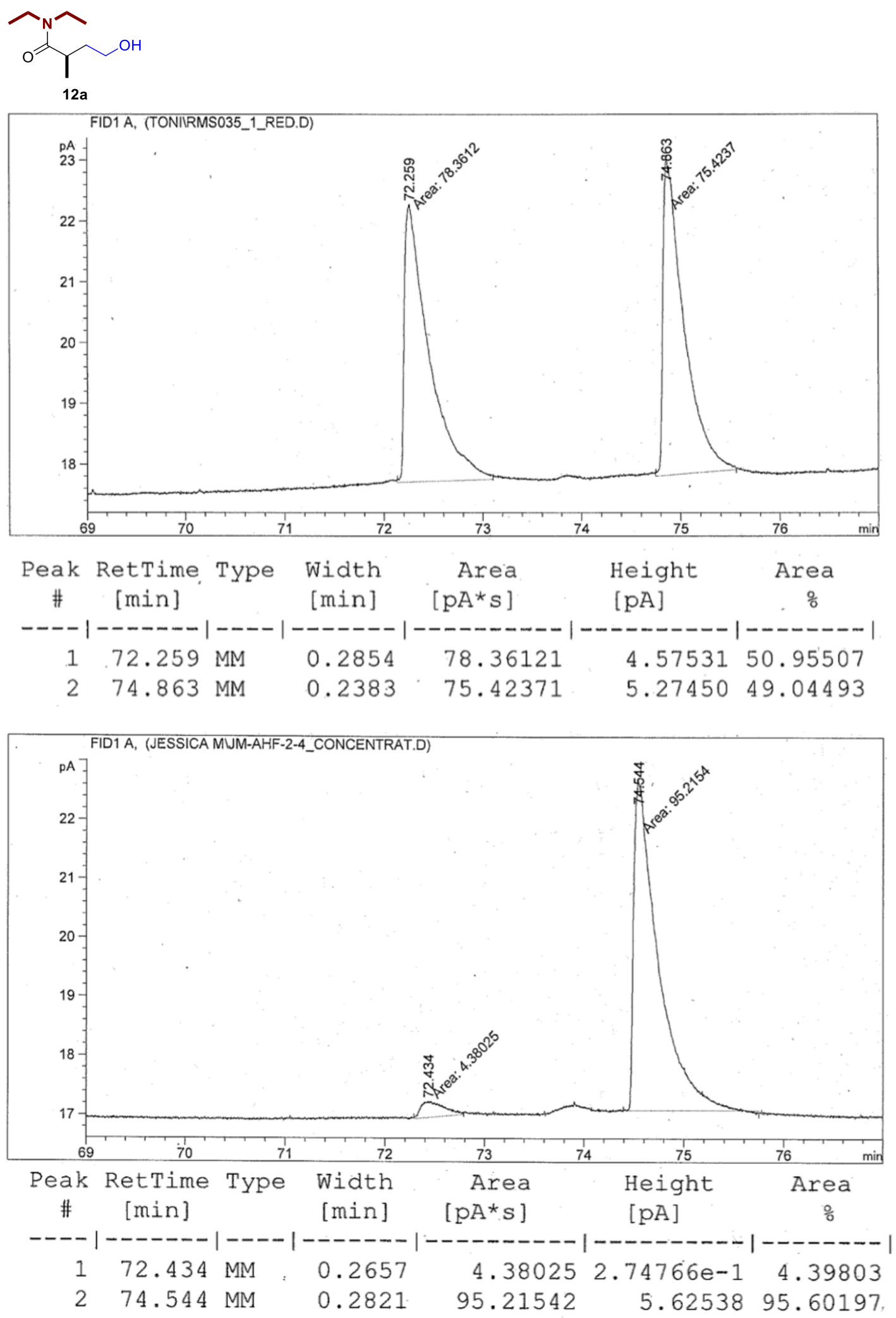

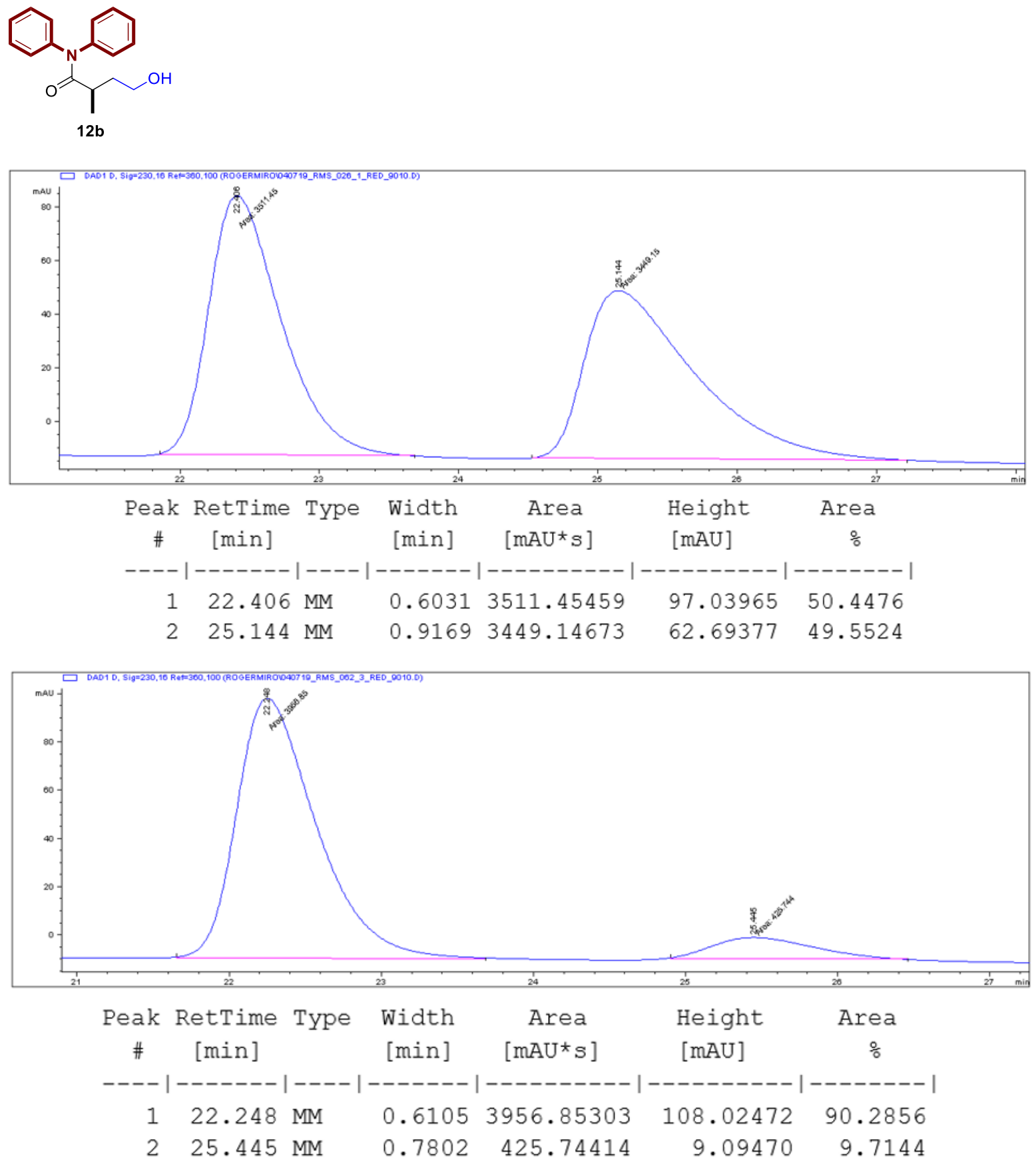

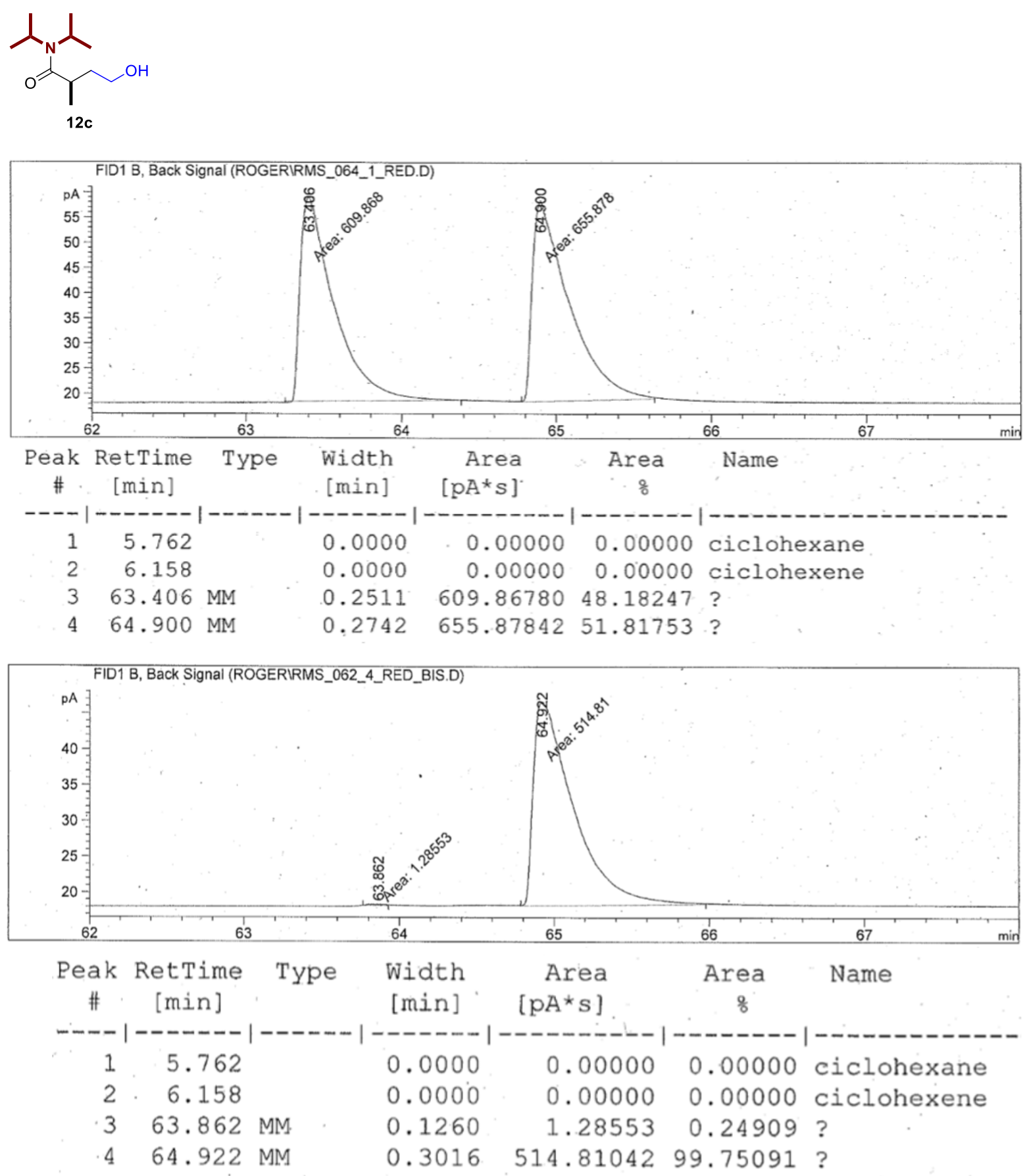

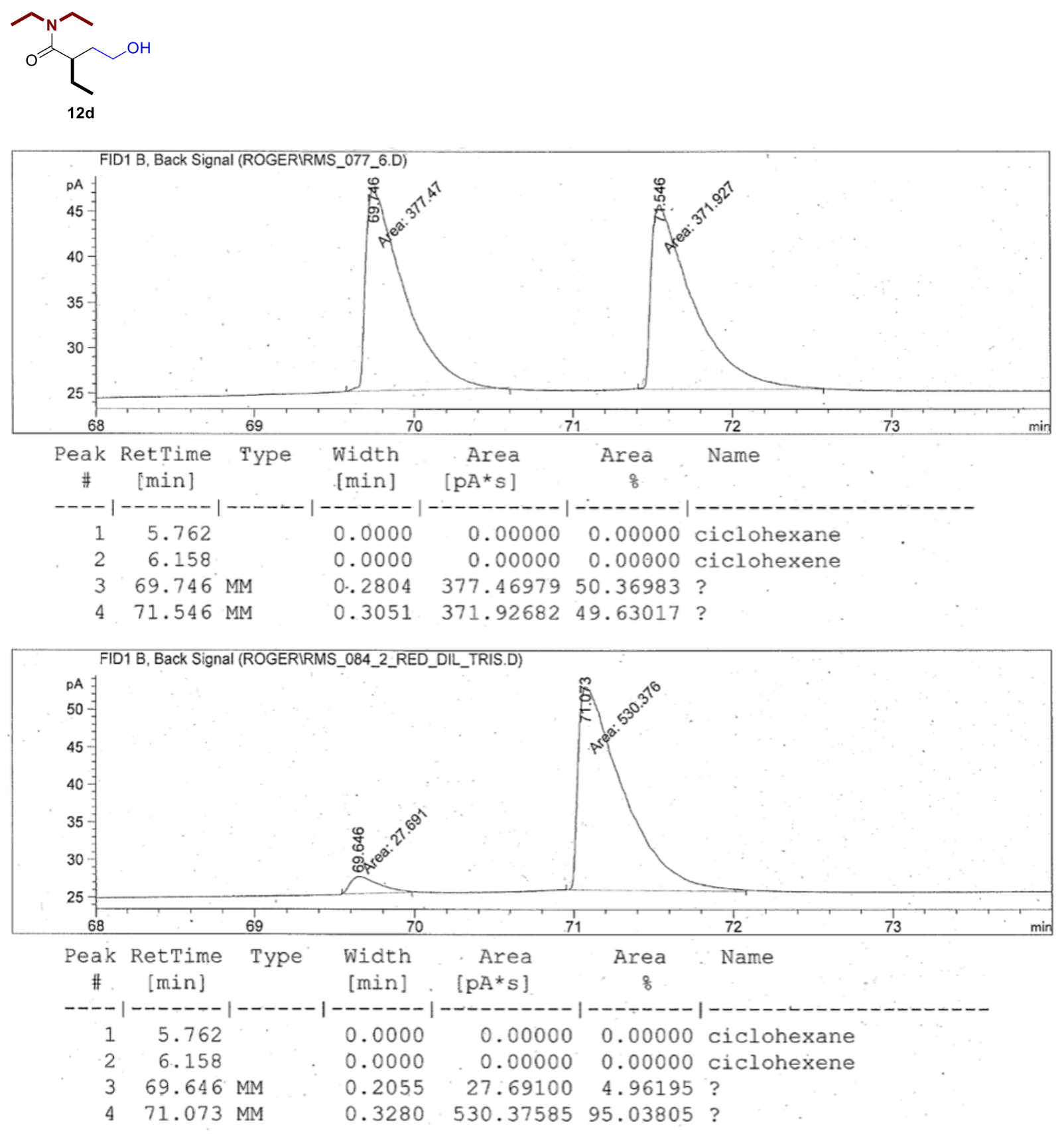

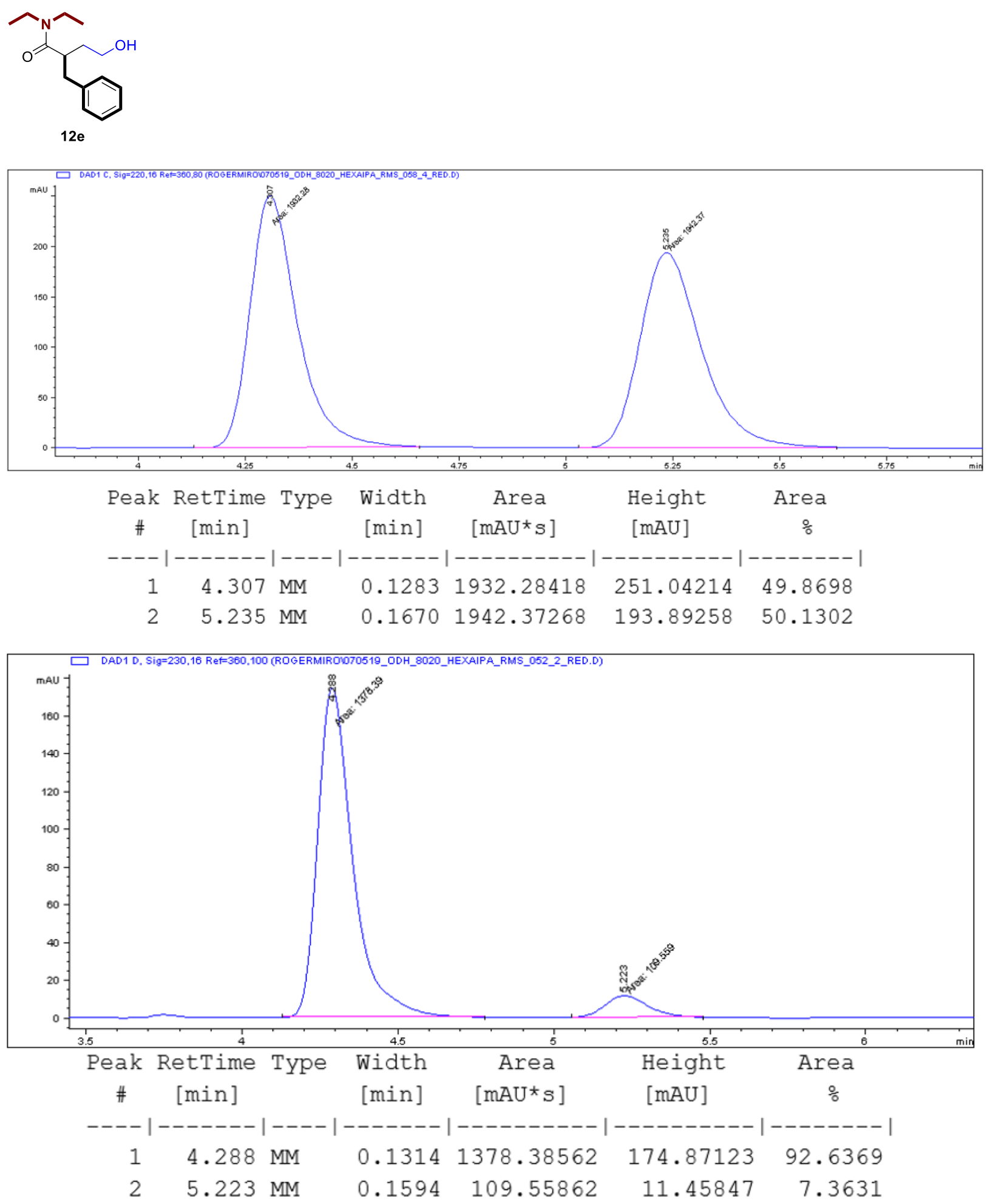

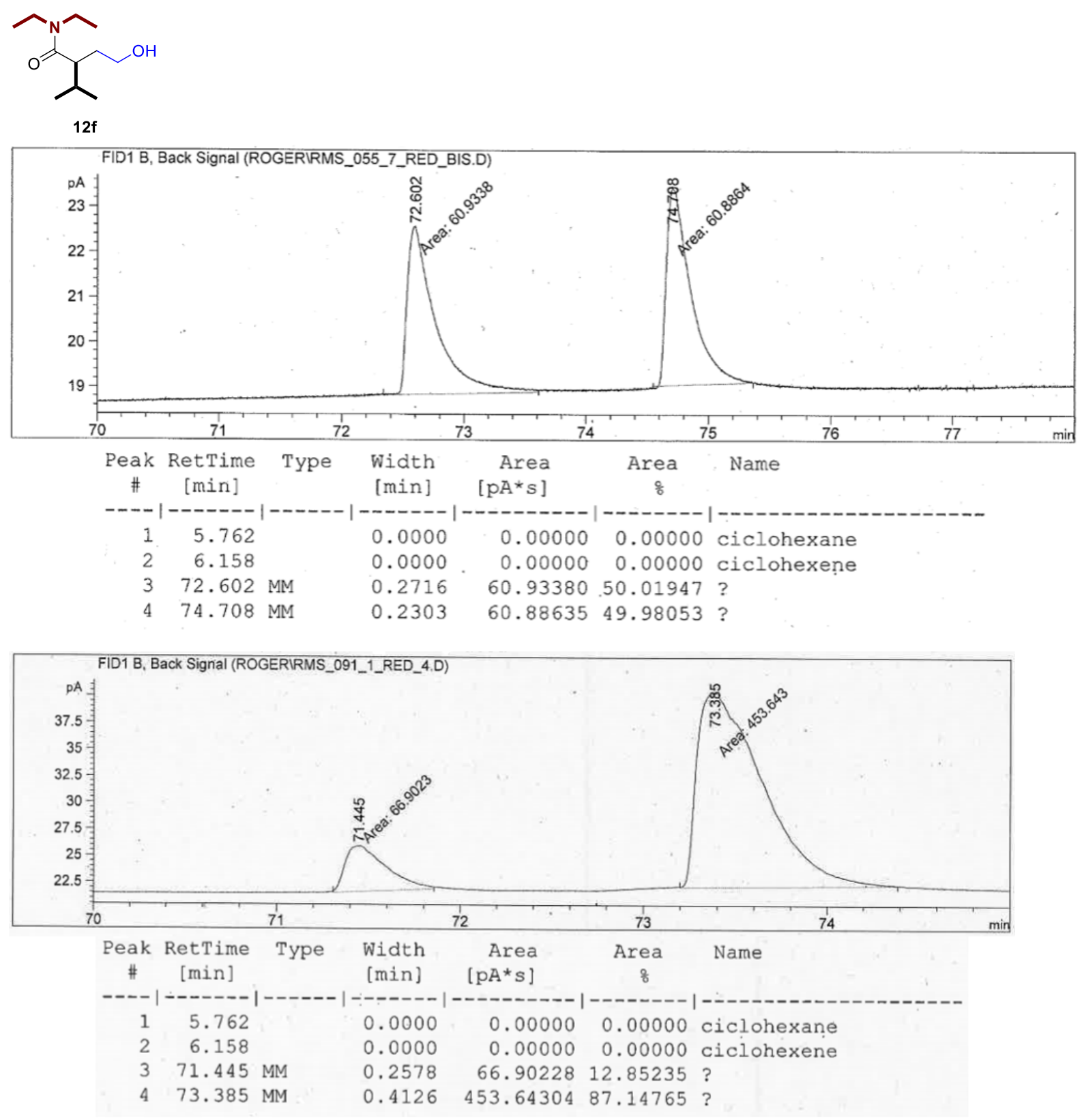

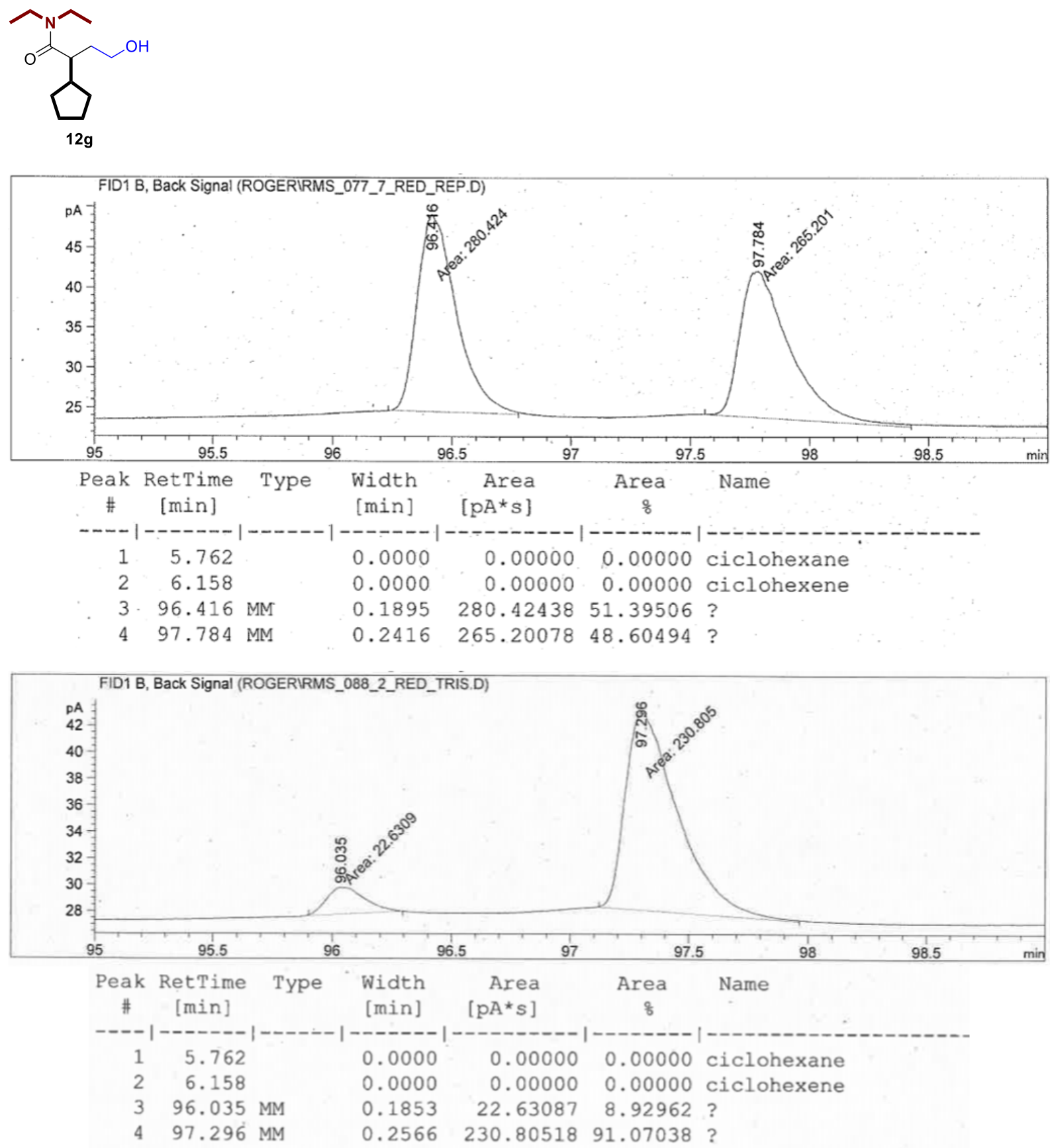

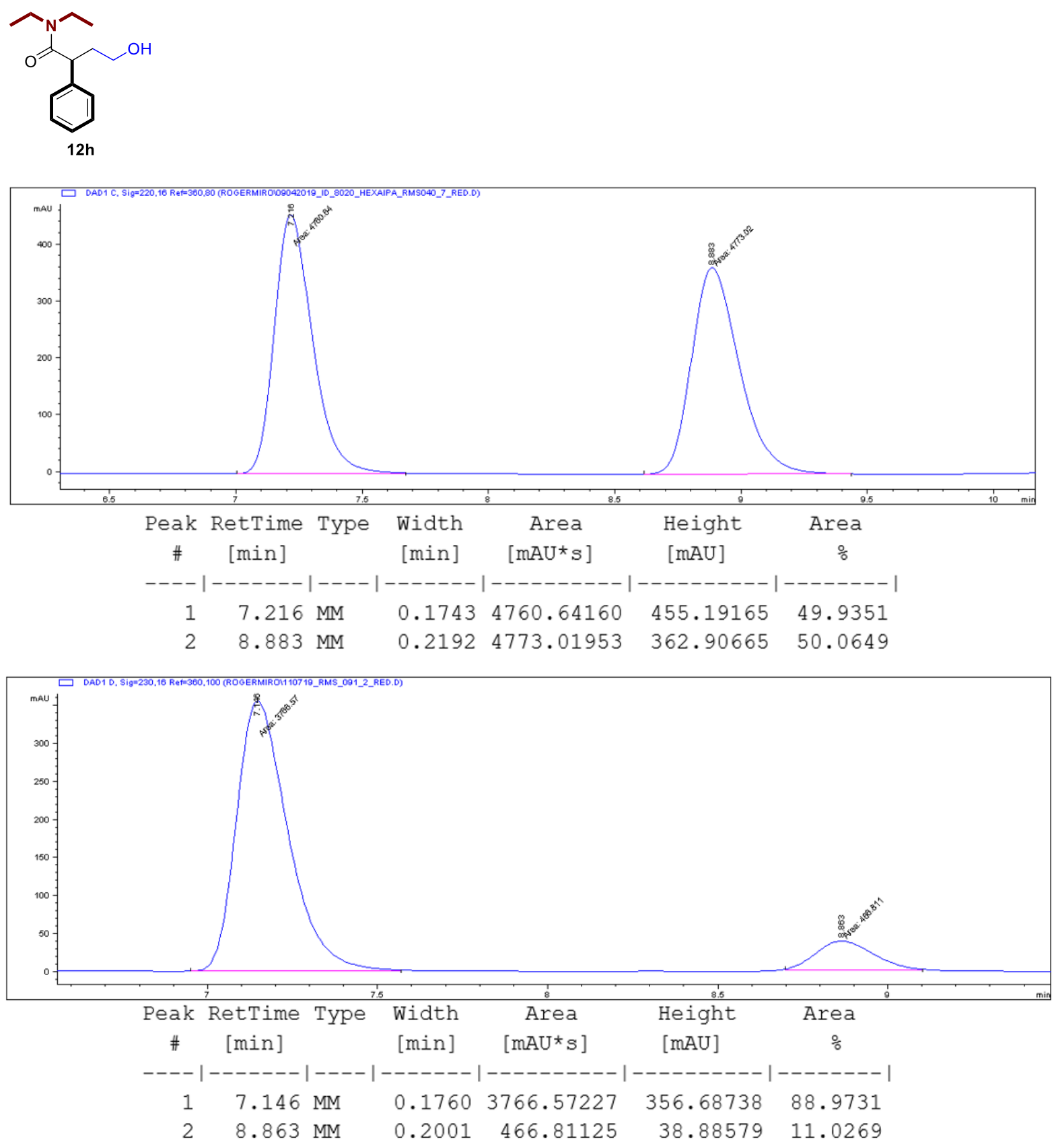

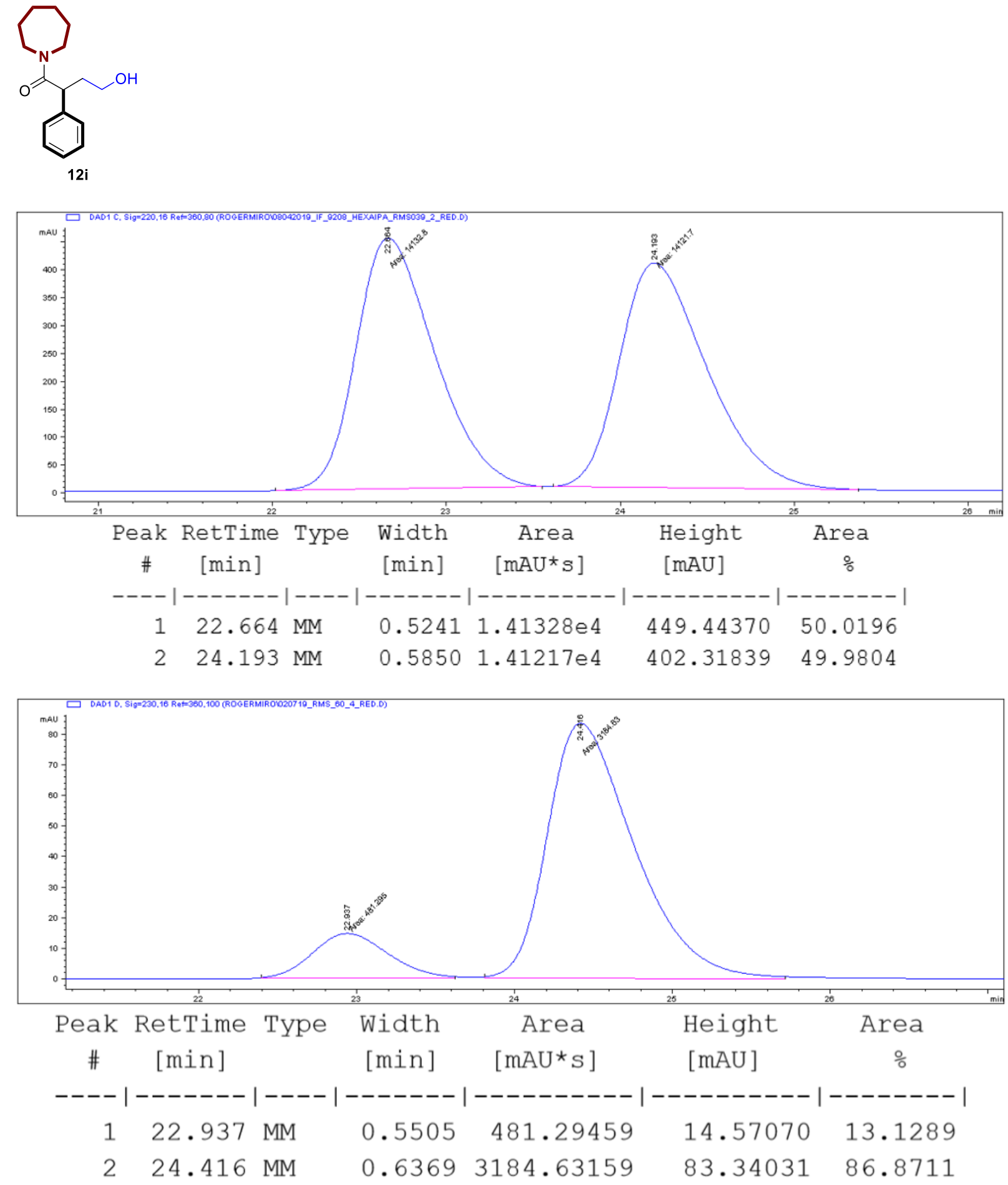
b. HPLC $\alpha$-alkyl-y-aminobutyric amides traces<smiles>CCN(CC)C(=O)C(C)CCN1CCOCC1</smiles>

$4 a$
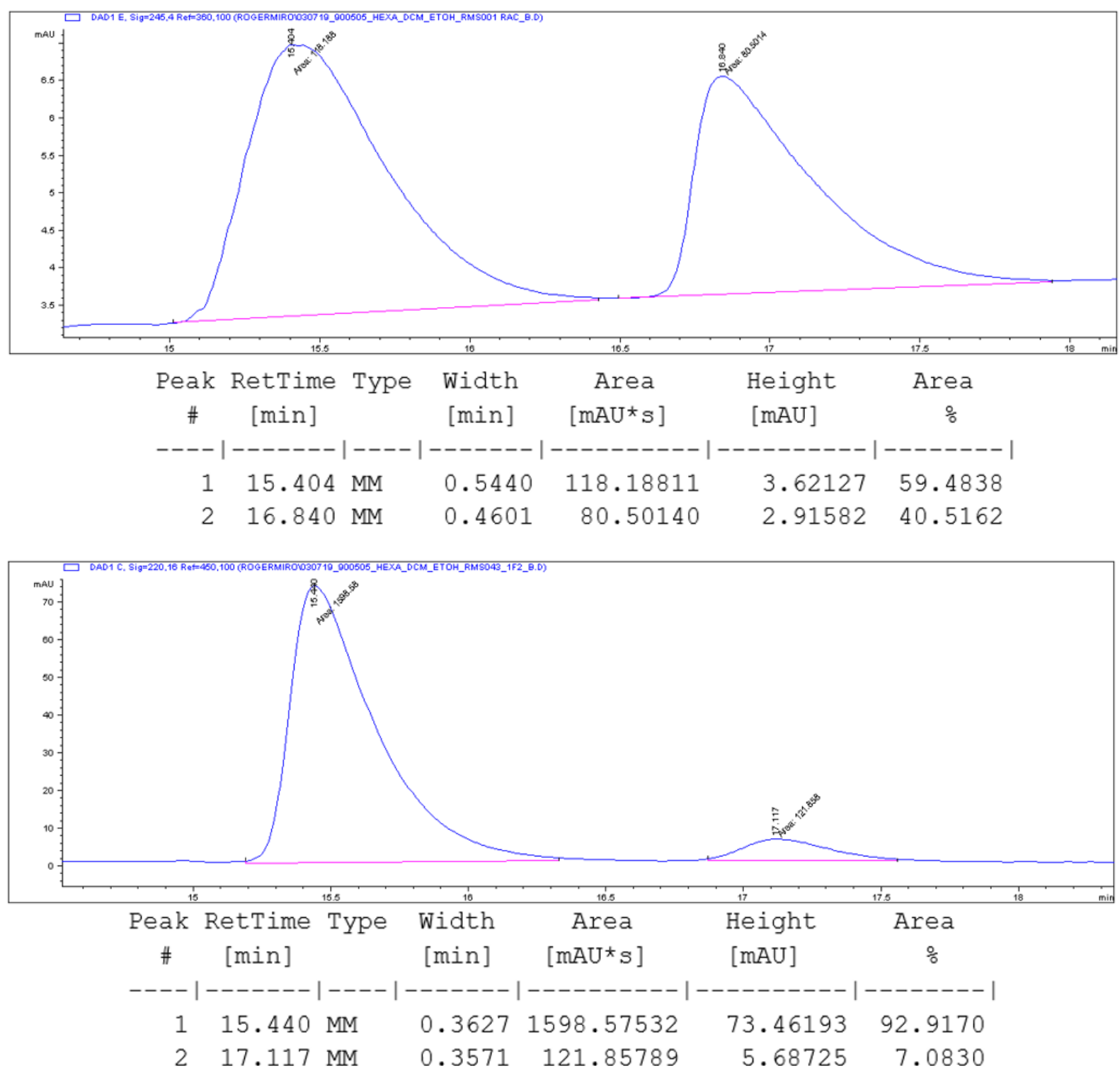
<smiles>CCN(CC)C(=O)C(C)CCN1CCN(C(=O)OC(C)(C)C)CC1</smiles>

$4 b$
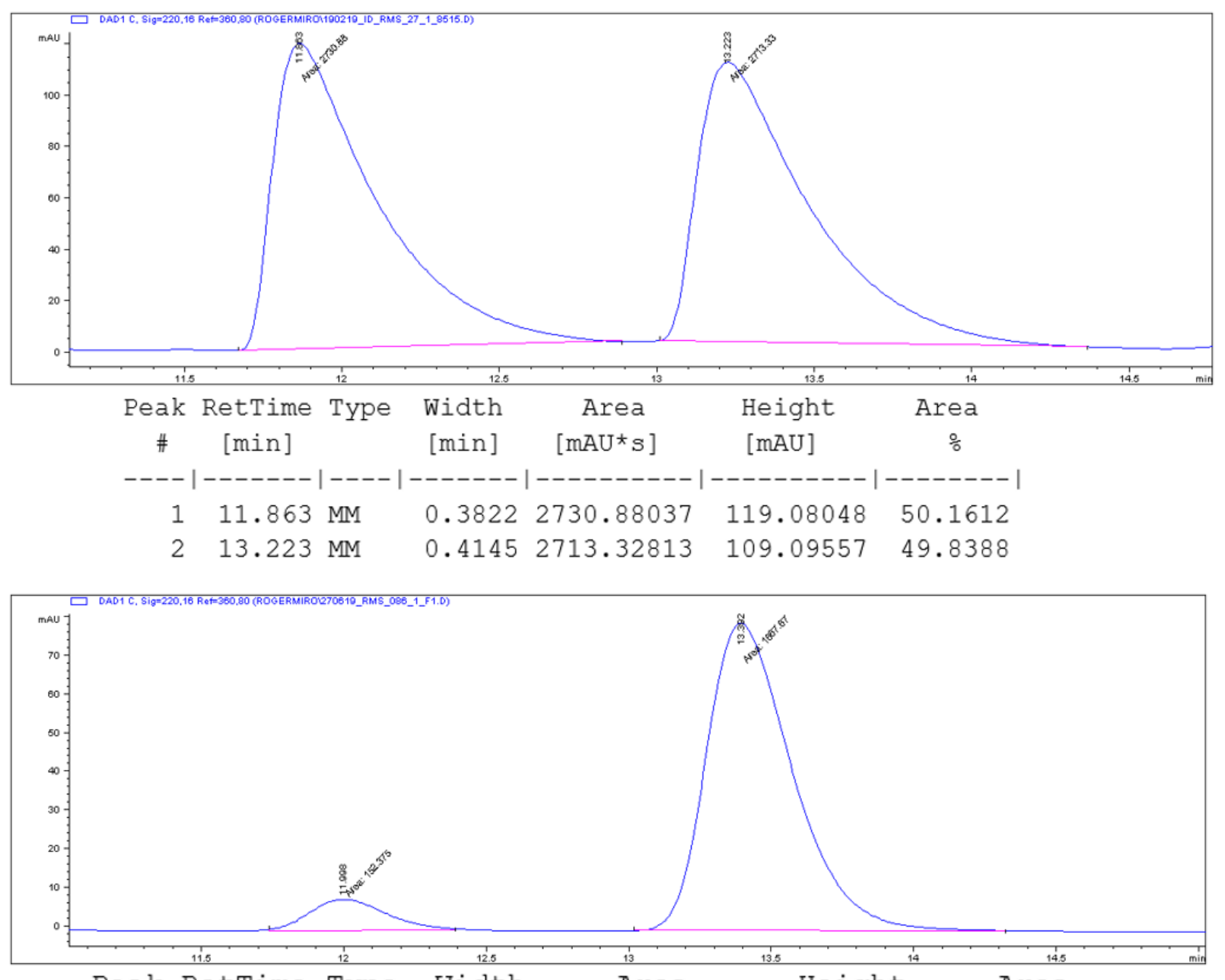

\begin{tabular}{|c|c|c|c|c|c|c|}
\hline $\begin{array}{c}\text { Peak } \\
\text { \# }\end{array}$ & $\begin{array}{c}\text { RetTime } \\
\text { [min] }\end{array}$ & Type & $\begin{array}{l}\text { Width } \\
\text { [min] }\end{array}$ & $\begin{array}{c}\text { Area } \\
{\left[\mathrm{mAU}{ }^{*} \mathrm{~S}\right]}\end{array}$ & $\begin{array}{l}\text { Height } \\
\text { [mAU] }\end{array}$ & $\begin{array}{c}\text { Area } \\
\frac{\circ}{\circ}\end{array}$ \\
\hline & & & & & & \\
\hline 2 & 13.392 & $\mathrm{MM}$ & 0.3495 & 1667.66882 & 79.53518 & 91.6279 \\
\hline
\end{tabular}




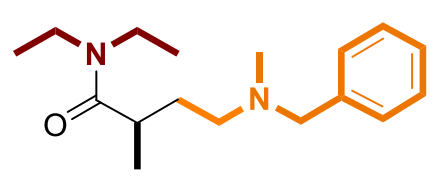

4c
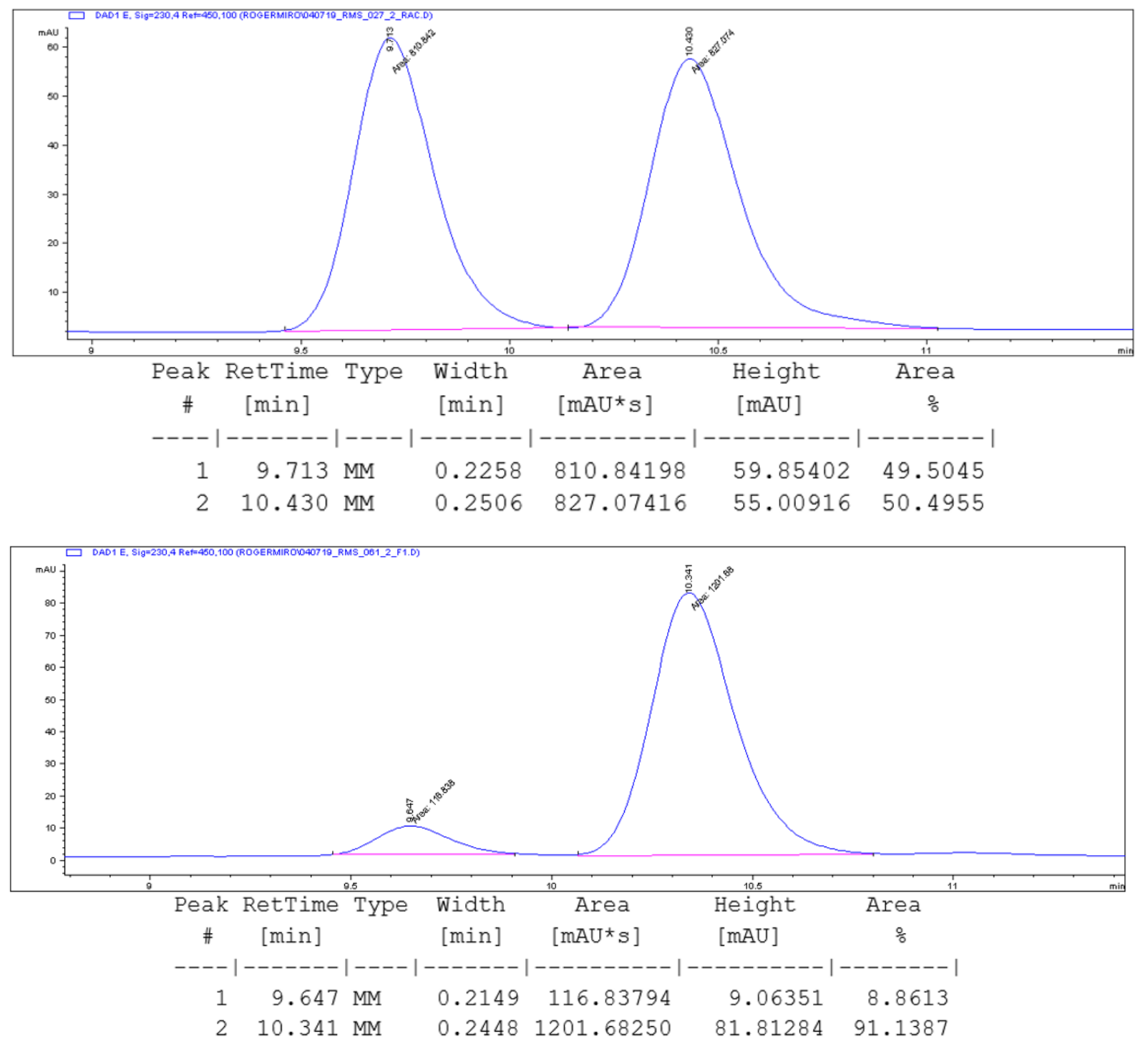
<smiles>CCN(CC)C(=O)C(C)CCN1CCN(c2ccccc2OC)CC1</smiles>

4d
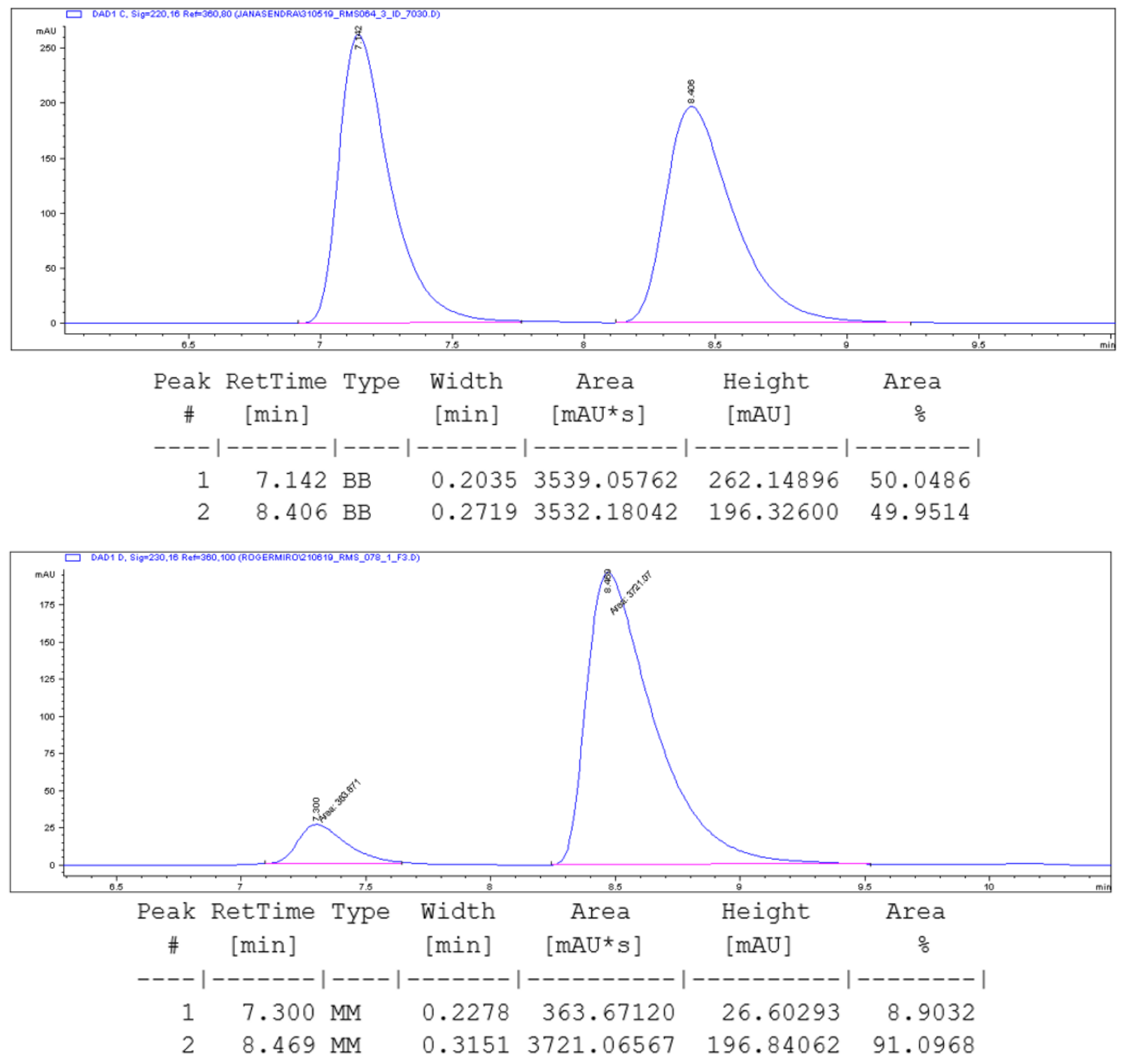

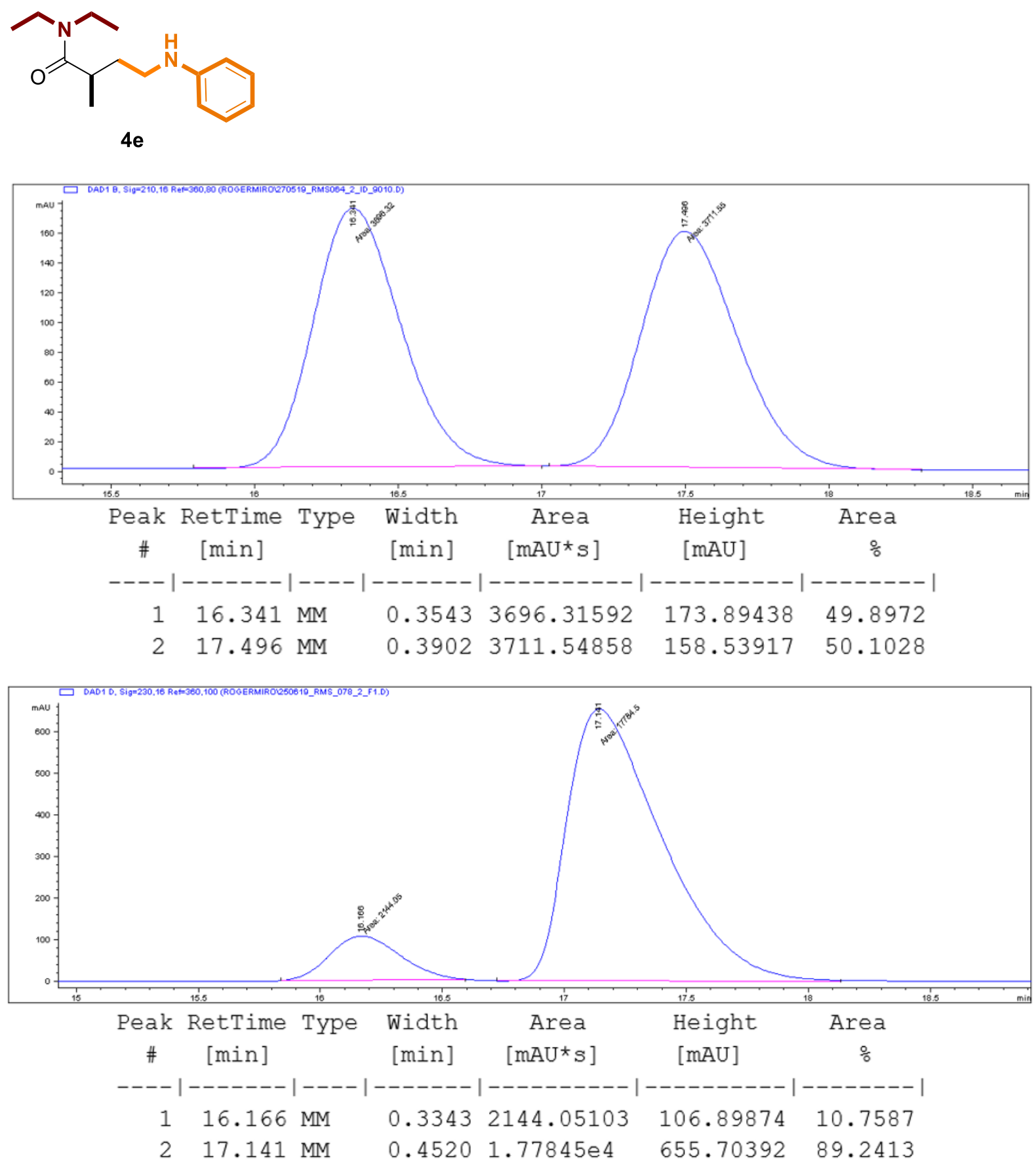
<smiles>CC(CCN1CCOCC1)C(=O)N(C(C)C)C(C)C</smiles>

$4 f$
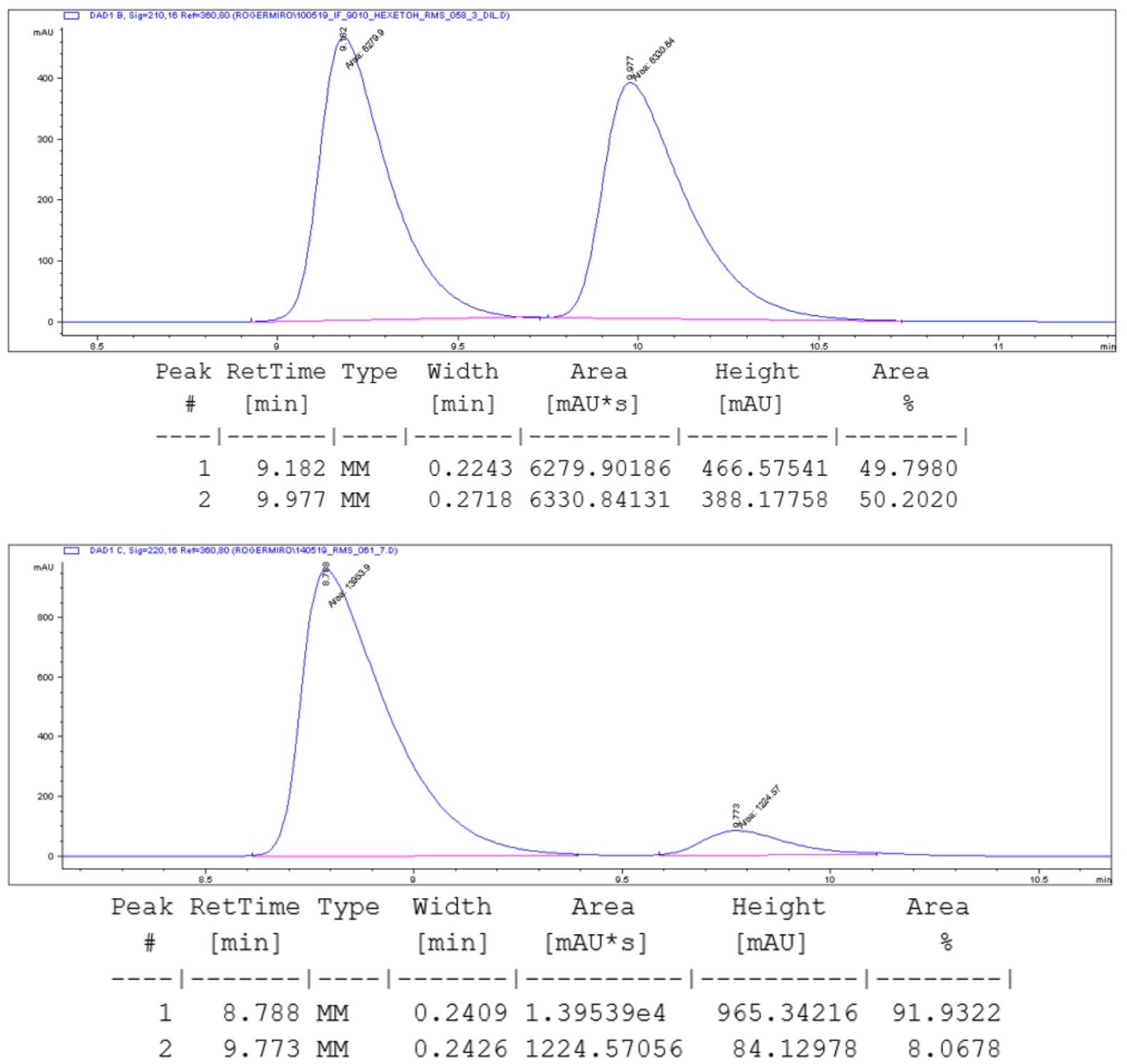


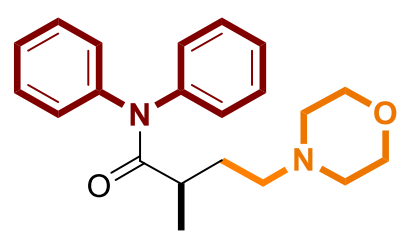

4g
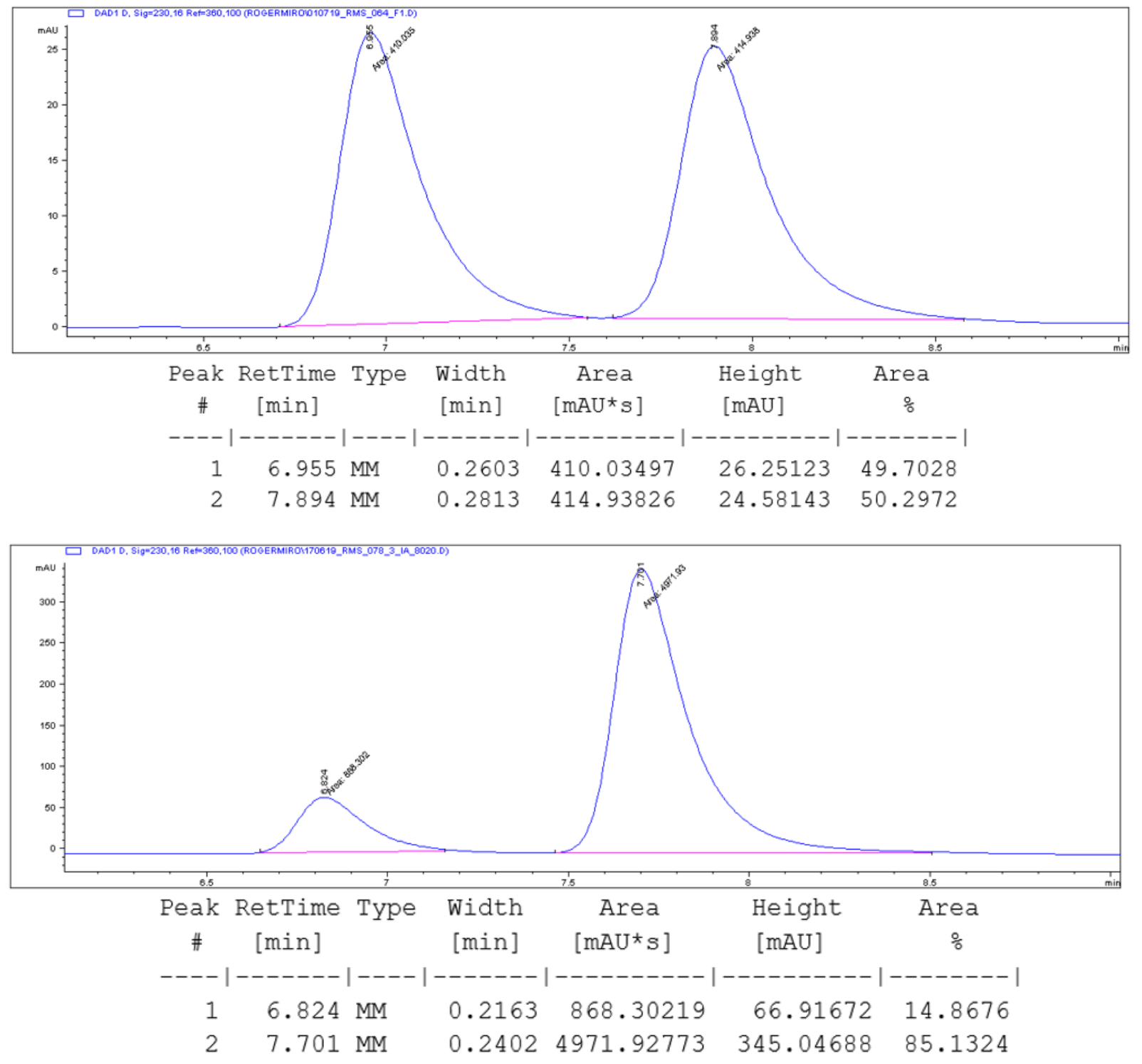
<smiles>CCC(CCN1CCOCC1)C(=O)N(CC)CC</smiles>

4h
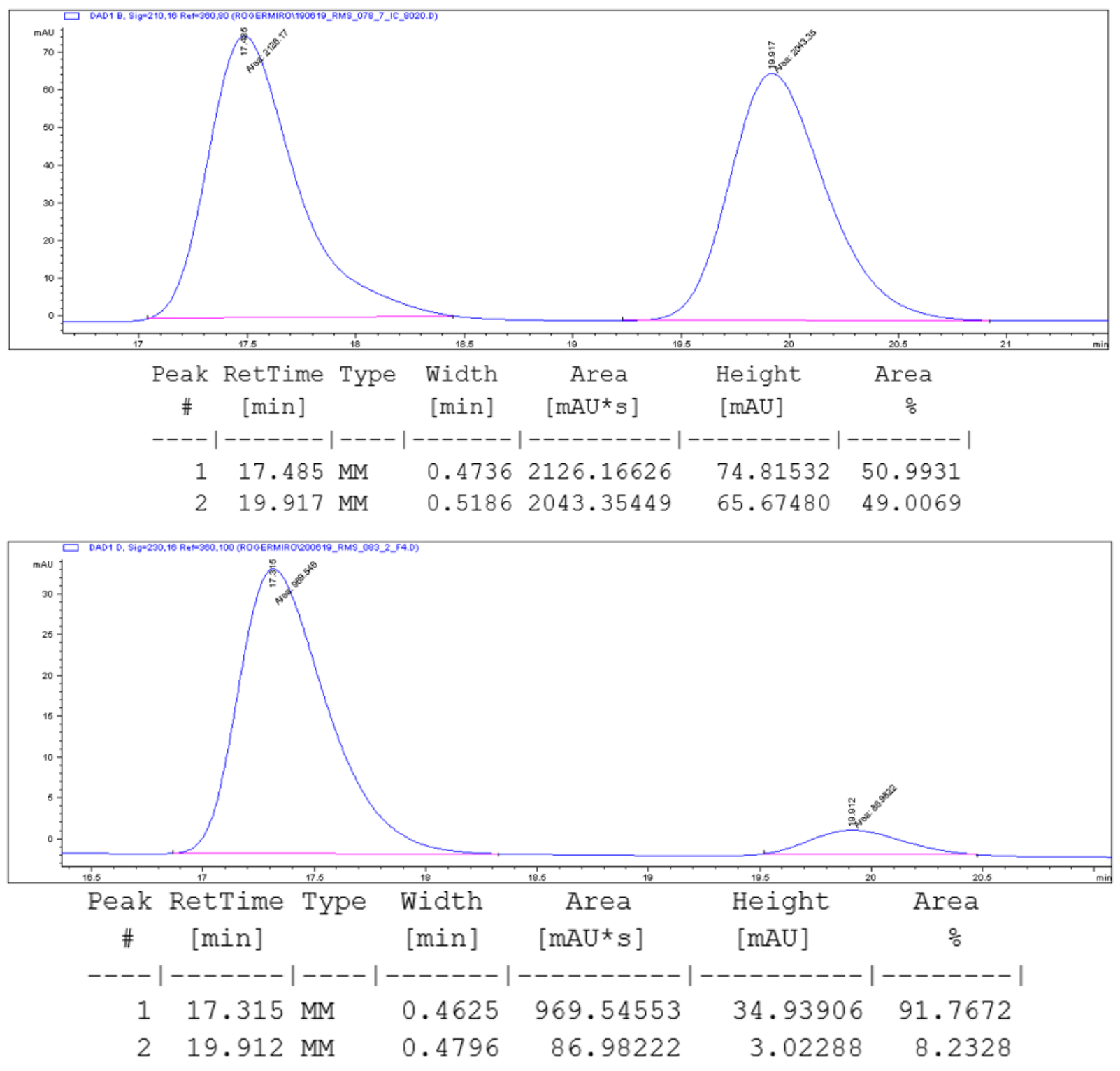
<smiles>CCN(CC)C(=O)C(CCN1CCOCC1)Cc1ccccc1</smiles>
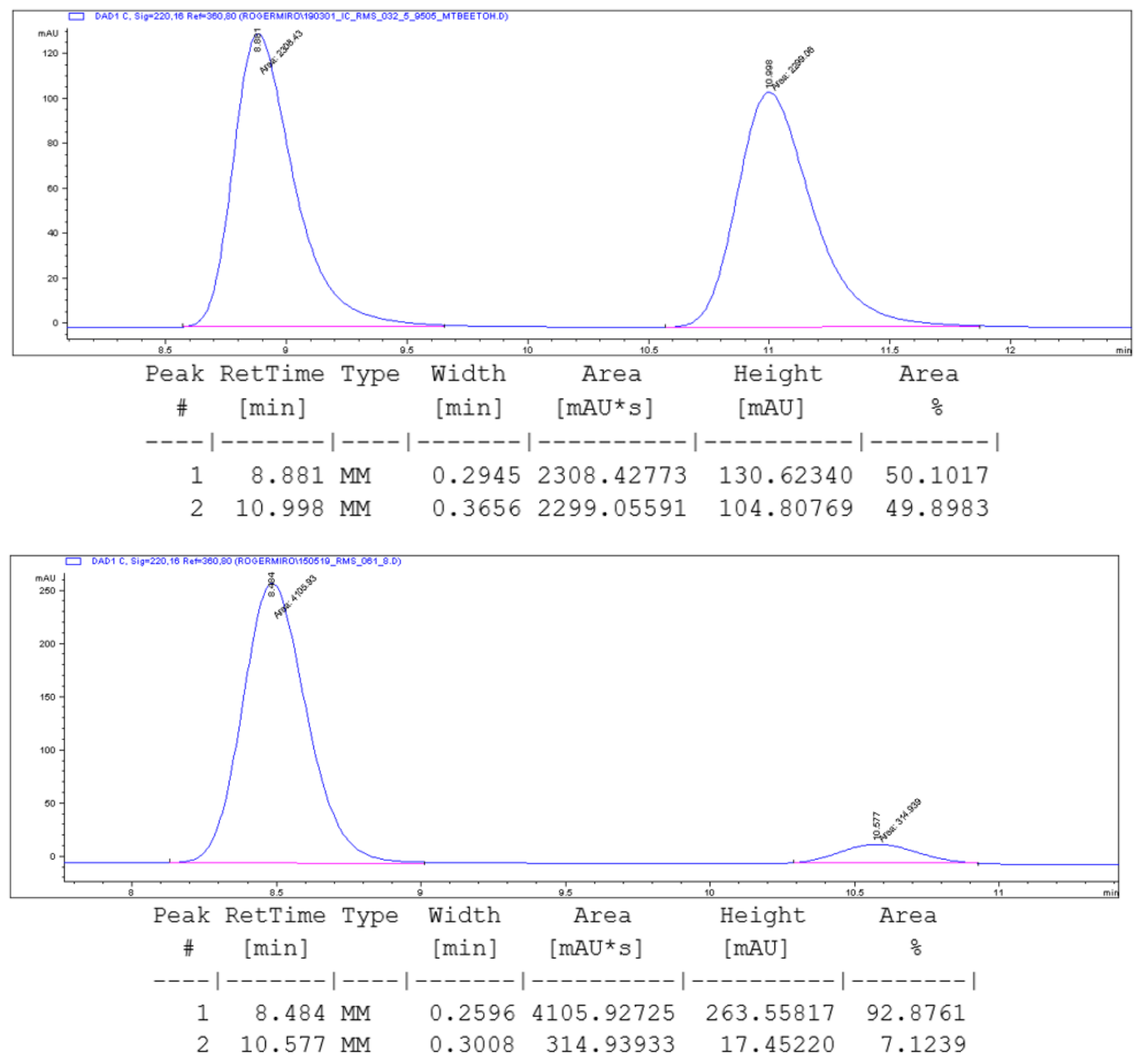

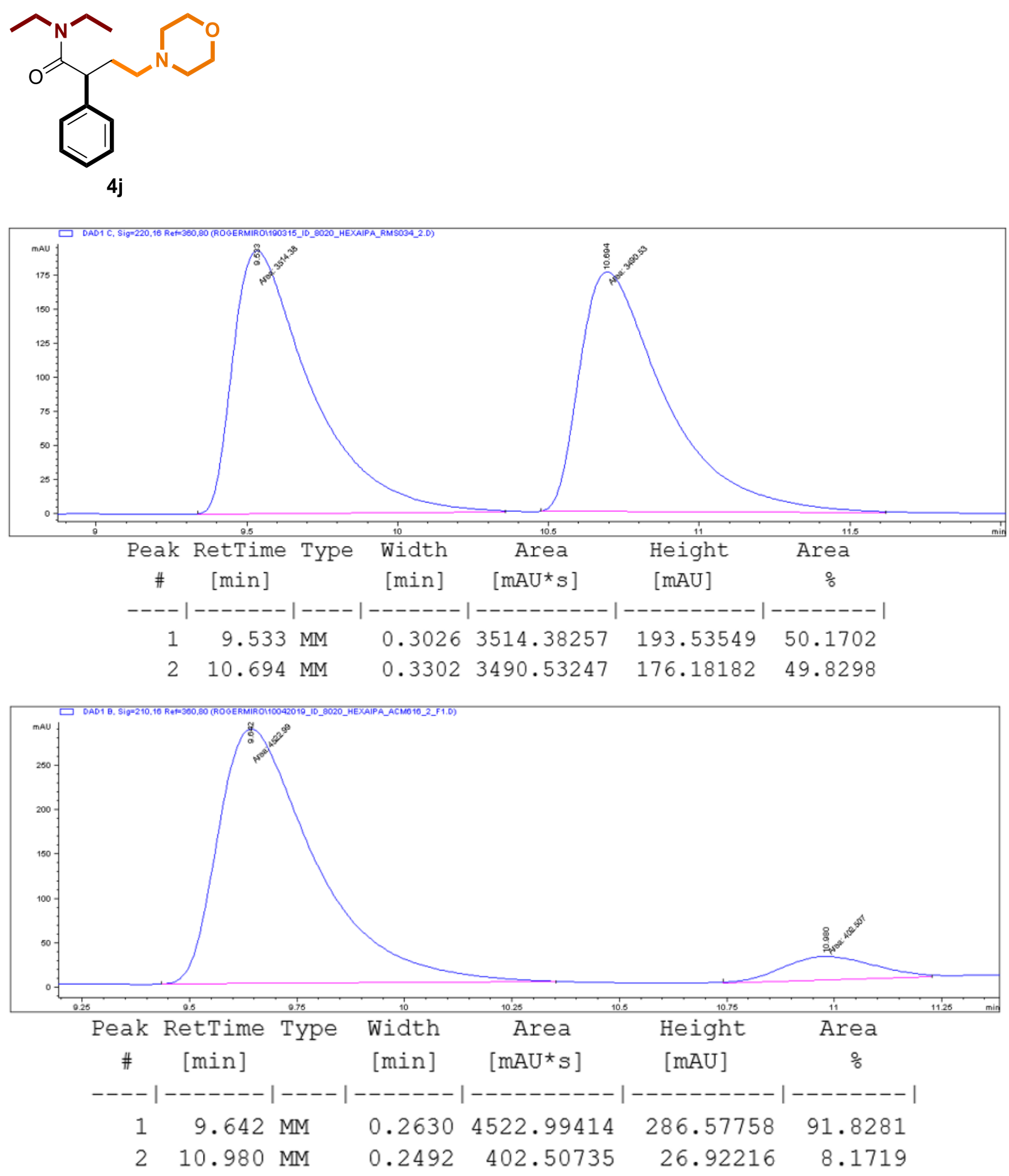

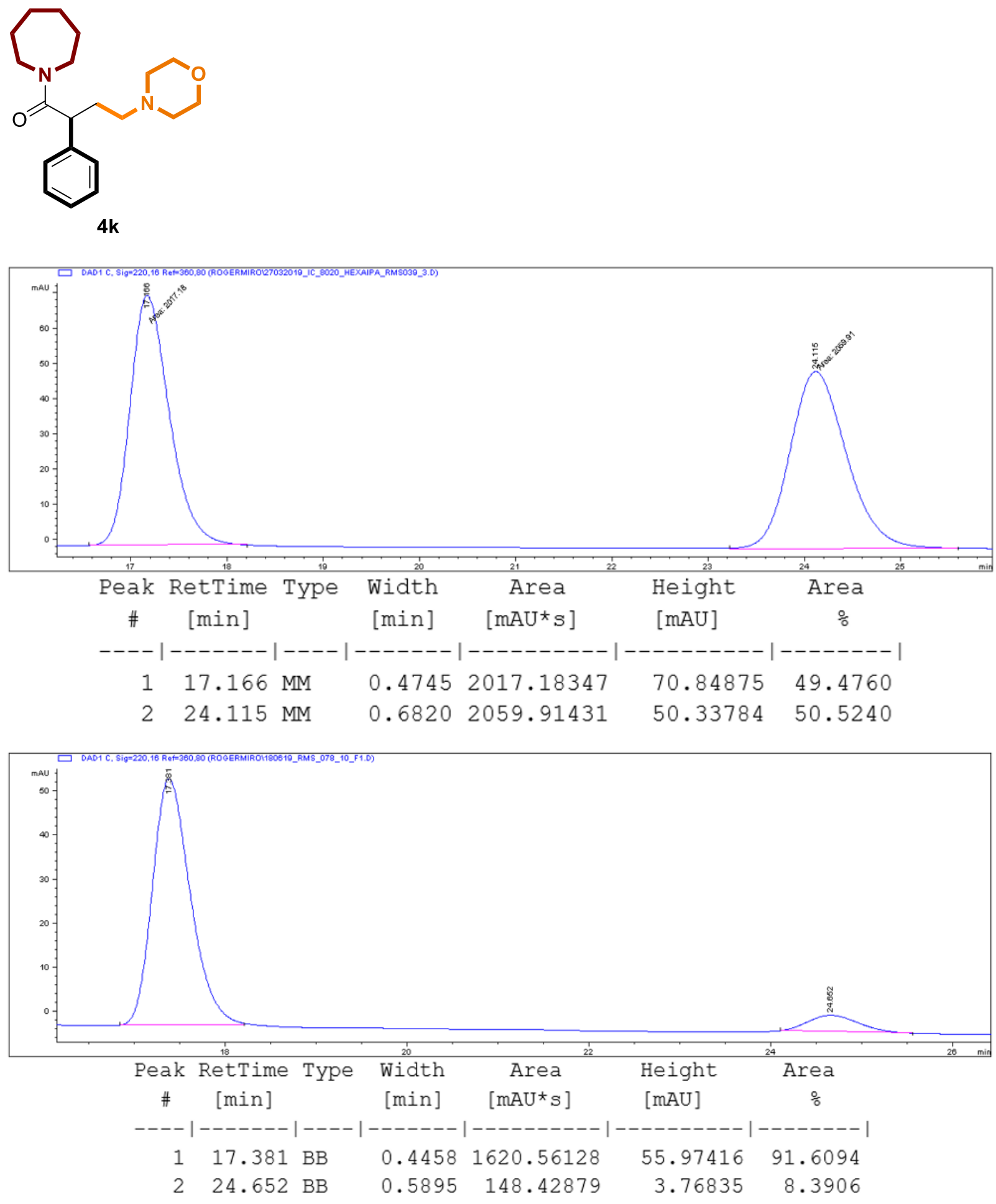

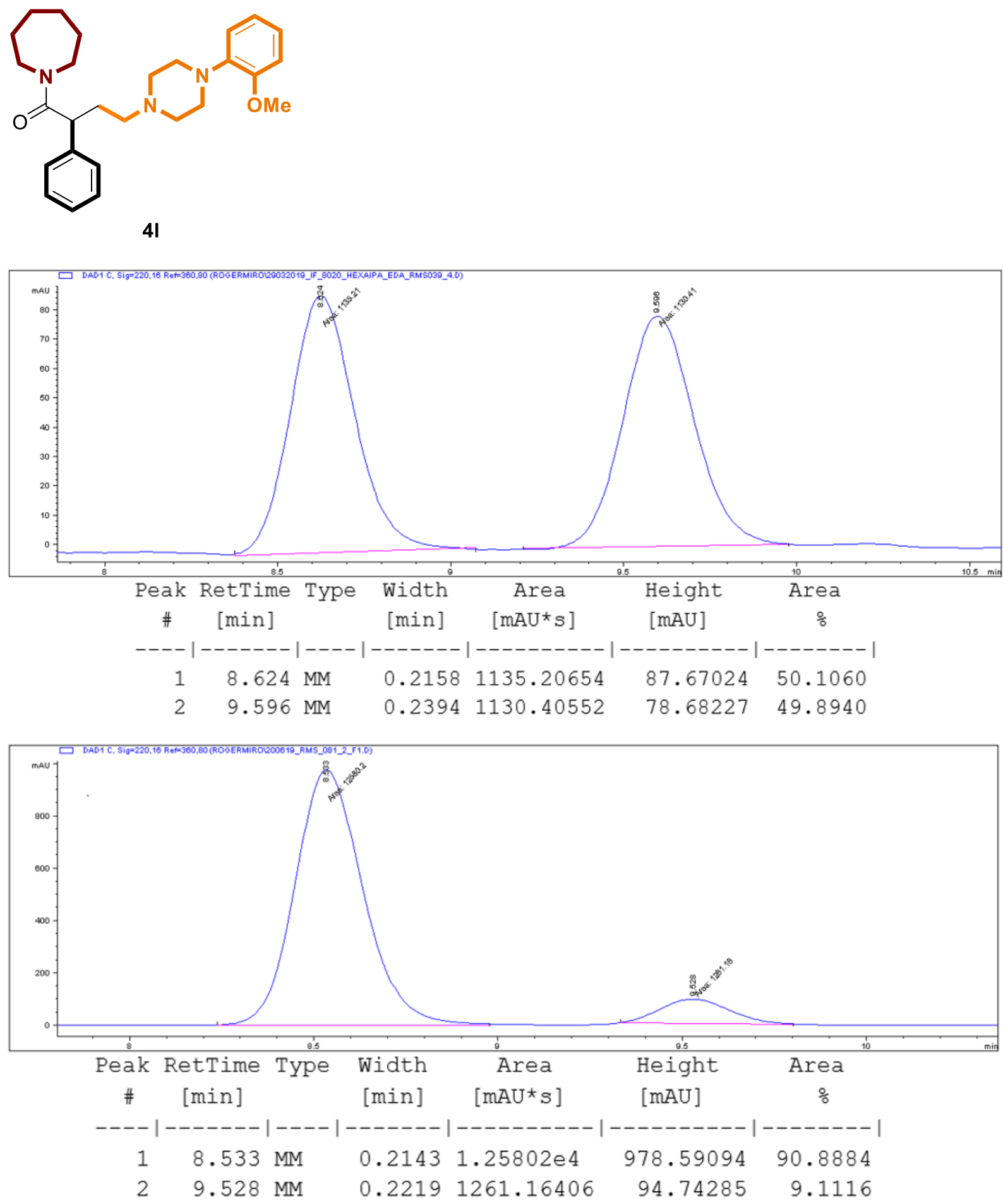


\section{SXX. References}

${ }^{1}$ Gual, A.; Godard, C.; Castillón, S.; Claver, C. Adv. Synth. Catal. 2010, 352, 463-477.

${ }^{2}$ Ferreira, S. B.; Sodero, A. C. R.; Cardoso, M. F. C.; Lima, E. S.; Kaiser, C. R.; Silva Jr, F. P.; Ferreira, V. F. J. Med. Chem. 2010, 53, 2364-2375.

${ }^{3}$ Cho, B. T.; Kim, N. J. Chem. Soc., Perkin Trans. 1 1996, 2901-2907.

4 Domke, L. (2015) Asymmetric hydrogenation and hydroformylation of 1,1-disubstituted olefins (PhD thesis); University of Rostock.

${ }^{5}$ Farley, A.; Sandford, C.; Dixon, D. J. Am. Chem. Soc. 2015, 137, 15992-15995.

${ }^{6}$ Wencel-Delord, J.; Nimphius, C.; Patureau, F.; Glorius, F. Chem. Asian J. 2012, 7, 1208-1212.

${ }^{7}$ Lu, K.; Han, X.; Yao, W.; Luan, Y.; Wang, Y.; Chen, H.; Xu, X.; Zhang, K.; Ye, M. ACS Catal. 2018, 8, 3913-3917.

${ }^{8}$ Fu, M.; Chen, L.; Jiang, Y.; Jiang, Z.; Yang, Z. Org. Lett. 2016, 18, 348-351.

${ }^{9}$ Fañanás, F. J.; Hoberg, H. J. Organomet. Chem. 1984, 275, 249-56.

${ }^{10}$ Zhao, Q.; Tognetti, V.; Joubert, L.; Besset, T.; Pannecoucke, X.; Bouillon, J.; Poisson, T. Org. Lett. 2017, 19, 2106-2109.

${ }^{11}$ McCarron, J.; Zoghbi, S.; Shetty, H.; Vermuelen, E.; Wikström, H.; Ichise, M.; Yasuno, F.; Halldin, C.; Innis, R.; Pike, V. Eur. J. Nucl. Med. Mol. Imaging, 2007, 34, 1670-1682. 